\title{
A physicochemical approach to design bioactive scaffolds for tissue engineering
}

Citation for published version (APA):

Chen, H. (2017). A physicochemical approach to design bioactive scaffolds for tissue engineering.

[Doctoral Thesis, Maastricht University]. Maastricht University. https://doi.org/10.26481/dis.20170406hc

Document status and date:

Published: 01/01/2017

DOI:

10.26481/dis.20170406hc

Document Version:

Publisher's PDF, also known as Version of record

\section{Please check the document version of this publication:}

- A submitted manuscript is the version of the article upon submission and before peer-review. There can be important differences between the submitted version and the official published version of record.

People interested in the research are advised to contact the author for the final version of the publication, or visit the DOI to the publisher's website.

- The final author version and the galley proof are versions of the publication after peer review.

- The final published version features the final layout of the paper including the volume, issue and page numbers.

Link to publication

\footnotetext{
General rights rights.

- You may freely distribute the URL identifying the publication in the public portal. please follow below link for the End User Agreement:

www.umlib.nl/taverne-license

Take down policy

If you believe that this document breaches copyright please contact us at:

repository@maastrichtuniversity.nl

providing details and we will investigate your claim.
}

Copyright and moral rights for the publications made accessible in the public portal are retained by the authors and/or other copyright owners and it is a condition of accessing publications that users recognise and abide by the legal requirements associated with these

- Users may download and print one copy of any publication from the public portal for the purpose of private study or research.

- You may not further distribute the material or use it for any profit-making activity or commercial gain

If the publication is distributed under the terms of Article $25 \mathrm{fa}$ of the Dutch Copyright Act, indicated by the "Taverne" license above, 


\section{A Physicochemical Approach to Design Bioactive Scaffolds for Tissue Engineering}

Honglin Chen 


\section{Supervisors:}

Prof.dr. C.A. van Blitterswijk

Prof.dr. L. Moroni

Co-supervisors:

Dr. R.K. Truckenmüller

A Physicochemical Approach to Design Bioactive Scaffolds for Tissue Engineering by Honglin Chen

PhD Thesis, Maastricht University, Maastricht, The Netherlands

ISBN: 978-94-6233-600-1

The research described in this thesis was funded by China Scholarship Council.

The printing of this thesis was sponsored in part by:

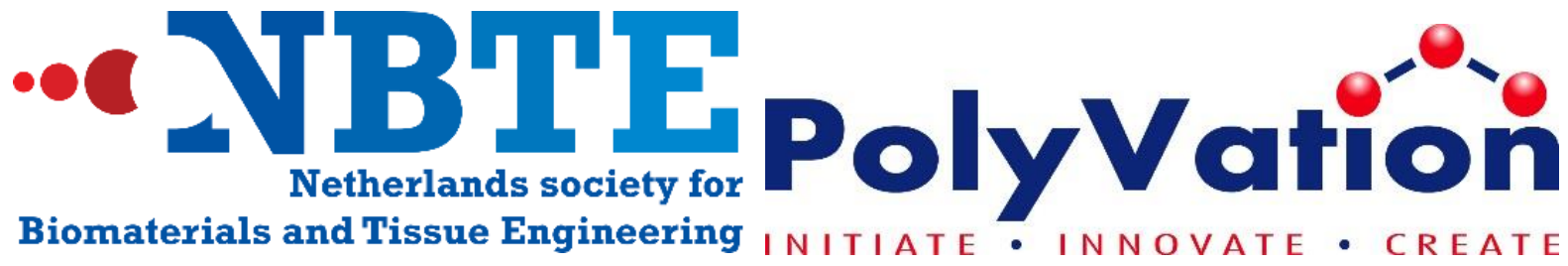

Copyright ( Honglin Chen, Maastricht, The Netherlands, 2017. Neither this book nor its parts may be reproduced without written permission of the author.

Printed by: Gildeprint, Enschede, The Netherlands

Cover Art: The cover was designed by the author, adapted from "Bricklayer" by www.redocn.com. 


\section{A Physicochemical Approach to \\ Design Bioactive Scaffolds for Tissue Engineering}

DISSERTATION

To obtain the degree of Doctor at the Maastricht University,

on the authority of the Rector Magnificus,

Prof.dr. Rianne M. Letschert

In accordance with the decision of the Board of Deans,

to be defended in public

on Thursday, April $6^{\text {th }} 2017$, at $14: 00$

by

Honglin Chen

Born on September $27^{\text {th }}, 1985$

In Fujian, China 


\section{Member of the committee:}

\section{Promotors:}

Prof.dr. C.A. van Blitterswijk

Maastricht University

Prof.dr. L. Moroni

Maastricht University

Co-promotor:

Dr. R.K. Truckenmüller

Maastricht University

\section{Assessment Committee:}

Prof.dr. S. Jockenhoevel (Chairman) RWTH Aachen University/Maastricht University

Prof.dr. H. Fischer RWTH Aachen University

Prof.dr. S. Rastogi Maastricht University

Prof.dr. L.W. van Rhijn Maastricht University

Dr. Fang Yang, Radboud University Prof.dr. P. Habibovic Maastricht University Prof.dr. H. Yuan Sichuan University

The names of assessment members are listed in random order. 


\section{Table of Contents}

\section{Chapter 1}

General Introduction and Aims

Chapter 2

Tailoring Chemical and Physical Properties of Fibrous Scaffolds from Block Copolyesters Containing Ether and Thio-ether Linkages for Skeletal Differentiation of Human Mesenchymal Stromal Cells

\section{Chapter 3}

Flexible Yttrium-Stabilized Zirconia Nanofibers Offer Bioactive Cues for

Osteogenic Differentiation of Human Mesenchymal Stromal Cells

Chapter 4

Tailoring Surface Nanoroughness of Electrospun Scaffolds for Skeletal

Tissue Engineering

Chapter 5

From Fiber Curls to Mesh Waves: a Platform for the Fabrication of

Hierarchically Structured Electrospun Fibers Mimicking Natural Tissue Formation

\section{Chapter 6}

Direct Writing Electrospinning of Scaffolds with Multi-dimensional Fiber

Architecture for Hierarchical Tissue Engineering

\section{Chapter 7}

General Discussion

Chapter 8

Valorisation

Summary

Acknowledgements

Biography 



\section{Chapter 1}

\section{General Introduction and Aims}




\section{Abstract}

Tissue engineering aims at developing biological substitutes to restore maintain or improve tissue function. Scaffold-based tissue engineering provides an alternative approach for tissue regeneration. This approach involves the combination of cells and/or biomolecules with scaffolds, which serve as a temporary substrate for cells adhesion and a physical support to guide new tissue formation. Nanofibrous scaffolds mimicking the structural features of natural extracellular matrix (ECM) can be appealing scaffold candidates for tissue engineering as they provide similar physical cues to the native environment of the targeted tissue to regenerate. This chapter discusses different strategies to fabricate nanofibrous scaffolds for tissue engineering. We first describe three major methods for nanofibrous scaffolds fabrication: molecular self- assembly, phase separation, and electrospinning. Then, approaches for surface modification of nanofibrous scaffolds including blending and coating, plasma treatment, wet chemical methods, and surface graft polymerization are presented. Finally, applications of nanofibrous scaffolds in tissue engineering are introduced and the synopsis of this thesis described.

\section{Key words:}

Electrospinning, self-assembly, phase separation, nanofibrous scaffolds, tissue engineering 


\subsection{Introduction}

Tissue engineering aims at developing biological replacements through the integration of life science and engineering principles. In general, tissue engineering can be subdivided into three major approaches [1]: (1) injection or transplantation of isolated cells to a defect site or an injured tissue, (2) delivery of tissue-inducing biomolecules (e.g. growth factors, peptides, polysaccharides) to a targeted tissue, (3) growth and differentiation of specific cell types in three dimensional (3D) scaffolds. Among these approaches, scaffold-based tissue engineering has become the most popular, due to the potential of current scaffold fabrication technologies to incorporate chemical, physical, and biological stimuli at different scales able to direct cell activity.

The extracellular matrix (ECM) usually provides structural support to the cells in addition to performing various other important functions. Many extracellular proteins have a fibrous structure with a diameter at the nanometer or sub-micrometer scales. For example, collagen, the most abundant ECM protein in our body, has a fibrous structure with a fiber diameter that ranges from 50 to $500 \mathrm{~nm}$. For successful and functional engineering of tissues and organs in scaffold-based approaches, an artificial scaffold should mimic the structure and biological function of native ECM as much as possible both in terms of physical cues and chemical composition. Therefore, researchers have put much effort to develop nanofibrous scaffolds that mimic the fibrillar structure of native ECM. Currently, there are three basic techniques available for generating nanofibrous scaffolds: molecular self-assembly, phase separation, and electrospinning (Table 1). Although these techniques allow developing nanofibrous scaffolds that mimic ECM physical structure with a number of degradable and non-degradable synthetic polymers, additional modifications are desirable to present biological cues, which exhibit a positive effect on cell adhesion, proliferation, and differentiation. Furthermore, recent studies have shown that submicron and nanoscale topographies can also modulate cell activity, thus acting as "synthetic" biological cues [2]. Surface modifications of nanofibrous scaffolds include blending and coating, plasma treatment, wet chemical methods, and surface graft polymerization. 
Table 1. Comparison of nanofiber processing techniques

\begin{tabular}{|c|c|c|c|c|c|}
\hline Method & $\begin{array}{l}\text { Level of } \\
\text { productivity }\end{array}$ & $\begin{array}{l}\text { Ease of } \\
\text { processing }\end{array}$ & Repeatability & Advantages & Disadvantages \\
\hline Self-assembly & $\begin{array}{l}\text { Laboratory } \\
\text { scale }\end{array}$ & Difficult & Yes & $\begin{array}{l}\text { Easy to get } \\
\text { fiber diameter } \\
\text { on lowest } \\
\text { scale } \\
\text { Great control } \\
\text { over 3D shape }\end{array}$ & $\begin{array}{l}\text { - } \text { Low yield } \\
\text { Complex } \\
\text { process } \\
\text { Little control } \\
\text { over fiber } \\
\text { dimension } \\
\text { and } \\
\text { orientation } \\
\text { Only short } \\
\text { fiber can be } \\
\text { obtained } \\
\text { Limited to a } \\
\text { few polymers }\end{array}$ \\
\hline $\begin{array}{l}\text { Phase- } \\
\text { separation }\end{array}$ & $\begin{array}{l}\text { Laboratory } \\
\text { scale }\end{array}$ & Easy & Yes & $\begin{array}{l}\text { Tailorable } \\
\text { mechanical } \\
\text { properties } \\
\text { Great control } \\
\text { over pore size } \\
\text { and shape } \\
\text { Great control } \\
\text { over 3D shape }\end{array}$ & $\begin{array}{l}\text { Low yield } \\
\text { Little control } \\
\text { over fiber } \\
\text { dimension } \\
\text { and } \\
\text { orientation } \\
\text { Difficult to } \\
\text { control pore } \\
\text { size and } \\
\text { shape } \\
\text { Limited to a } \\
\text { few polymers }\end{array}$ \\
\hline Electrospinning & $\begin{array}{l}\text { Laboratory/ } \\
\text { industrial } \\
\text { scales }\end{array}$ & Easy & Yes & $\begin{array}{l}\text { Well } \\
\text { established } \\
\text { and } \\
\text { characterized } \\
\text { technique } \\
\text { Long } \\
\text { continuous } \\
\text { fiber with } \\
\text { diameter from } \\
\text { micro scale } \\
\text { down to } \\
\text { nanoscale } \\
\text { Control over } \\
\text { fiber diameter } \\
\text { and } \\
\text { orientation } \\
\text { Tailorable } \\
\text { mechanical } \\
\text { properties } \\
\text { Plethora of } \\
\text { polymers may } \\
\text { be used }\end{array}$ & $\begin{array}{lr}\text { - } \begin{array}{l}\text { Difficult } \\
\text { fabricate }\end{array} \text { to } \\
\text { shape } \\
\text { Difficult to } \\
\text { control pore } \\
\text { size re and } \\
\text { shape }\end{array}$ \\
\hline
\end{tabular}

In this chapter, we illustrate nanofibrous scaffolds for tissue engineering. Processing technologies to achieve structural features resembling the ECM and approaches to improve cell-scaffold interactions will be introduced before discussing nanofibrous scaffolds for tissue engineering applications. 


\subsection{Methods for nanofibrous scaffolds fabrication}

\subsubsection{Molecular self-assembly}

Molecular self-assembly has emerged as a useful approach for manufacturing scaffolds for tissue engineering [3]. Unlike electrospinning, self-assembly can be defined as a spontaneous process to form ordered and stable structure through a number of noncovalent interactions, such as hydrophilic, electrostatic, and van deer Waals interactions $[4,5]$. The structural features of self-assembled materials can be tuned by controlling the kinetics, molecular chemistry, and assembling environment $(\mathrm{pH}$, solvent, light, salt addition and temperature). The key challenge in self-assembly is to design molecular building blocks that can undergo spontaneous organization into a well-defined pattern that mimic the structural features of biological systems [6]. Small building blocks including small molecules, nucleic acids, and peptides, can self-assemble into nanofibrous structures. Among these building blocks, peptide-amphiphile (PA) units, which combine the functions of peptides with the characteristics of surfactants, have gained a lot of attention due to the versatility in their design for biological applications. The chemical structure of a representative PA molecule consists of four key structure features: a hydrophobic domain consisting of a long alkyl tail, a short peptide sequence capable of forming intermolecular hydrogen bond, charged groups for enhanced solubility in water, and a bioactive epitope. Self-assembly of PA in water is governed by at least three major forces: hydrophobic interaction of alkyl tails, electrostatic repulsions between charged groups, and hydrogen bonding among peptide segments. The final structure of assembled PA reflects a balance of each force contribution [7].

The structural characteristics of nanofibers and nanofibrillar systems resulting from molecular self-assembly can be tailored by controlling processing parameters. Bundles of aligned PA nanofibers were obtained, for example, by self-assembling PA within parallel channels [8]. By introducing hydrophilic amino acids in peptide segments, specific sequences of PA could self-assemble into nanobelts with fairly monodisperse widths on the order of $150 \mathrm{~nm}$ and lengths of up to $0.1 \mathrm{~mm}$ that can be used as cell culture systems and drug delivery carriers [9]. Nanofibrous scaffolds were also fabricated from selfassembly of $\beta$-sheet peptides containing phenylalanine for controlled release [9, 10]. These studies proposed that the position of aromatic moieties is a significant determinant for supramolecular self-assembling conformations. Self-assembled chitin fibers with diameters of $3 \mathrm{~nm}$ and $10 \mathrm{~nm}$ could be also obtained from dissolving in hexafluoro-2propanol (HFIP) and $\mathrm{LiCl} / \mathrm{N}, \mathrm{N}$-dimethylacetamide (DMAC) respectively [11]. These 
observations demonstrated that nanofiber assembly occurs at a critical concentration and fiber morphology can be controlled by solution concentration and solution evaporation rate. Collagen-like nanofibers were self-assembled from collagen mimetic peptides by controlling triple-helical nucleation[12]. Young-Hag et al. fabricated macro/nano-porous collagen scaffolds comprising nanofibrous collagen filaments through 3D plotting in combination with self-assembly of collagen molecules [13]. Nanofibrous hydrogels, which were self-assembled from lysine-containing hexapeptides, had a stiffness of up to $40 \mathrm{kPa}$ and were biocompatible [14]. In addition, this self-assembling hydrogel could serve as an ideal bioink for bioprinting.

Molecular self-assembly is a fairly new technique to develop nanofibrous scaffolds which have been studied for a variety of tissue engineering applications including nerve [15], bone [16], and cartilage [17] regeneration. However, several technical hurdles still need to be addressed: First, it has not been demonstrated how to control the pore size and pore structure, which are important to allow for cell proliferation and migration. Secondly, their degradation profiles have not been systematically addressed [18]. Furthermore, most of self-assembled scaffolds are mechanically weak and do not effectively sustain and transfer mechanical loadings to the cells and surrounding tissues.

\subsubsection{Thermally induced phase separation}

Another interesting method used for manufacturing nanofibrous scaffolds is phase separation, which is a thermodynamic process involving the separation of phases due to physical incompatibility. Specifically, a homogeneous polymer solution becomes thermodynamically unstable under certain temperature conditions, and will form a polymer-rich phase and a polymer-poor phase. When the solvent is removed, the polymer-rich phase will solidify to a 3D structure while the polymer-poor phase will become the void space. The process of developing nanofibrous scaffolds from phase separation typically consists of 5 major steps: (i) raw material dissolution, (ii) gelation, (iii) solvent extraction, (iv) freezing and (v) drying [19]. The pore morphology of nanofibrous scaffolds can be tuned by varying processing parameters including polymer concentration, phase separation temperature and solvent/non-solvent exchange [20]. Furthermore, thermally induced phase separation can be combined with other processing techniques (e.g. particulate leaching and solid free-form fabrication) to generate scaffolds with complex porous structure and well-defined pore morphology [21]. For example, sodium chloride particles with diameters of 200-450 $\mu \mathrm{m}$ were mixed with a warm poly (lactic-coglycolic acid)/tetrahydrofuran (PLGA/THF) solution and then cooled to a preset gelation 
temperature. The formed composite gels were extracted with cold ethanol and washed with distilled water to remove the solvent and leach the salt particles. The samples were freeze-dried, resulting in a nanofirous scaffold with a macroporous structure left behind from the leached salt [22]. Nanofibrous poly(l-lactic acid) (PLLA) scaffolds have been developed by using phase separation to improve cell seeding, distribution and mass transport [23]. When compared with solid pore-walled PLLA scaffolds, nanofibrous scaffolds allowed approximately a 2-fold increase in adhesion of osteoblast cells. More recently, novel injectable functional nanofibirous hollow microspheres (FNF-HMS) were fabricated using phase separation [24]. Transforming growth factor- $\beta 1$ mimicking peptide cytomodulin, and bone morphogenetic protein-2 mimicking peptide P24 were conjugated onto FNF-HMS separately. In vitro and in vivo studies demonstrated that the injectable cell carriers successfully induced rabbit bone marrow-derived mesenchymal stem cells differentiation toward chondrocytes and osteoblasts.

Thermally induced phase separation is a promising technique to develop nanofibrous scaffold with well-defined pore shape and pore size. Although this technique can be combined with other fabrication methods to control the final 3D structure, the drawbacks of this technique, such as little control over fiber orientation and diameter, long fabrication time, and lack of mechanical properties need to be addressed to further achieve a fine control of the resulting scaffolds at the macro, micro, and nano scales.

\subsubsection{Electrospinning}

Electrospinning is a versatile and well-established process capable of producing fibers with diameters down to the sub-micron or nanometer range [25]. A basic electrospinning setup (Figure $1 \mathrm{~A}$ ) includes a high voltage electric source with positive or negative polarity; a syringe pump with capillaries or tubes to carry the solution (or melt) from a syringe to the spinneret and a conducting collector [26]. Under the electric field, the pulling force overcomes the surface tension of the polymer solution (or melt) and create a charged jet that travels through the atmosphere allowing the solvent to evaporate, thus leading to the deposition of solid polymer fibers on the collector [26, 27]. The fiber formation and structure is affected by three general types of variable: solution properties (concentration, viscosity, conductivity, and surface tension), processing factors (applied potential, collection distance, emitting electrode polarity, and feed rate), and environmental parameters (temperature, relative humidity, and velocity of the surrounding air in the spinning chamber) [26, 28]. 
A

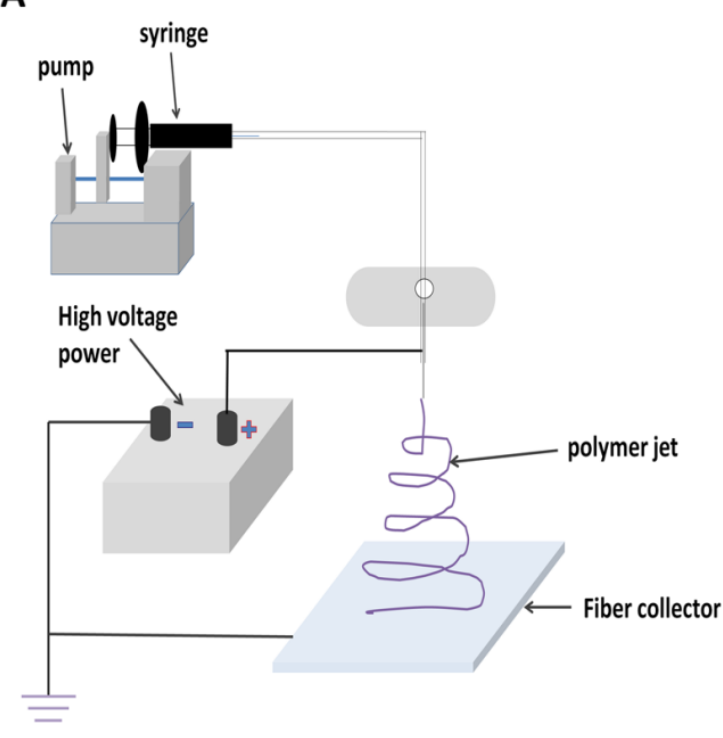

B
To pump

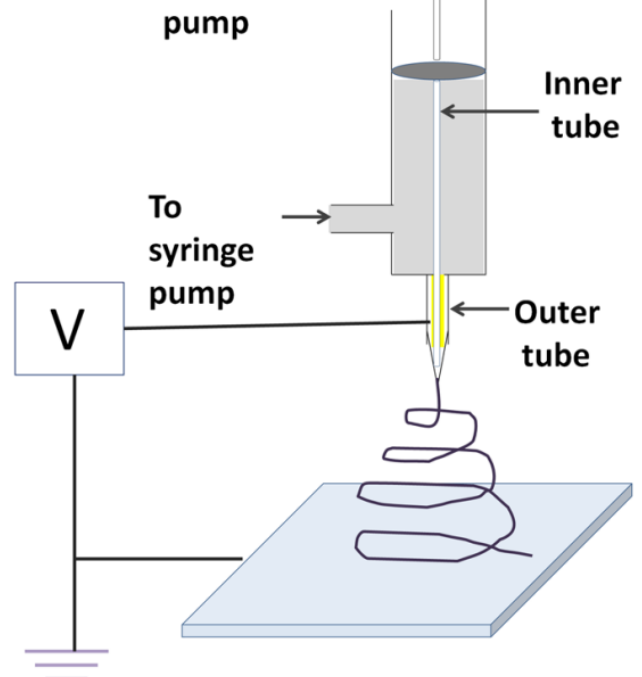

Figure 1. (A) Typical electrospinning set-up using a grounded static collector; (B) Configuration of the coaxial electrospinning setup used for preparing core-shell structured nanofibers.

For many applications, it is necessary to fabricate a scaffold made of aligned nanofibers, as the anisotropy in topography and structure can have a great impact on mechanical properties and cell behavior. Currently, a number of collecting devices have been developed to attain aligned electrospun fibers. These collecting devices can be divided into three main categories according to the type of forces involved [29]: (1) mechanical forces by using of a rotating mandrel; (2) electrostatic forces by using parallel electrodes; (3) magnetic forces by using parallel permanent magnets. Furthermore, electrospinning can be used for fabricating scaffolds with complex architectures such as stacked arrays and tubular conduits. Stacked arrays of nanofiber scaffolds can be achieved by multilayering electrospinning. Aligned nanofibers were stacked into multilayered films with a controllable hierarchical porous structure by depositing fibers on an insulating substrate (e.g. quartz) onto which an array of electrodes have been patterned [30]. Tubular conduits made of electrospun fibers can be fabricated by depositing fibers over a small diameter rod as a collector. These electrospun conduits are usually applied in vascular or neural tissue engineering since they mimic the structure of these tissues. For instance, electrospun fiber conduits were manufactured with a length of $12 \mathrm{~cm}$ and a thickness of $1 \mathrm{~mm}$ via electrospinning a mixture of collagen type I, elastin, and poly( $\mathrm{D}, \mathrm{L}$ lactide-co-glycolide) on a rotating rod of diameter $4.75 \mathrm{~mm}$ [31]. Compliance tests demonstrated that the electrospun fiber conduit possesses a diameter change of 
12 14\% within the physiologic pressure range, which exhibited a compliance behavior similar to that of a native vessel with a diameter change of $9 \%$. Burst pressure testing showed that the burst pressure for the electrospun fiber conduit was $1425 \mathrm{mmHg}$ or nearly 12 times that of normal systolic pressure.

In order to tailor the structures of resultant fibers, the modification of electrospnning set-ups have been carried out not only on the fiber collector but also on the spinneret. Coaxial spinnerets have been designed by many researchers for various aims in electrospinning (Figure 1.1B). Using this technology, core-shell nanofibers can be achieved. By using a spinneret with double coaxial capillaries, two components can be fed through different coaxial capillary channels to generate composite nanofibers in the form of core-sheath structure [32-34]. Some difficult-to-process polymer solutions can be also electrospun to form an ultrafine core within the shell of spinnable polymers. Thus, when the polymer sheath is removed, the desired polymer nanofber core is retained [35]. Using a similar concept, the core solution can be removed instead of the polymer sheath, resulting in hollow nanofibers. For example, an ethanol solution containing poly (vinyl pyrrolidone) (PVP) and tetraisopropyl titanate ( $\mathrm{Ti}(\mathrm{OiPr}) 4)$ was used as sheath solution, while mineral oil was used as core solution. After simultaneous ejection through the inner and outer capillaries, hollow nanofibers were formed through removal of the inner core miner oil phase [36]. Shell-core and hollow nanofibers have shown their potential in tissue engineering and drug delivery due to their unique architecture and properties. Biologically active agents can be encapsulated inside a polymer shell to form a reservoir-type drug delivery device. Therefore, the polymer shell would offer a temporal protection for the bioactive agents and control their sustain release [32]. Tissue regeneration processes can be also facilitated via introduction of biomolecules (e.g. growth factors) into the core of shell-core nanofibrous scaffolds and, eventually, the release of multiple factors can be envisioned by adding different compounds in the shell and in the core fibers.

Electrospun fibers with high surface to volume ratio and structures resembling extracellular matrix (ECM) have shown great potential applications in tissue engineering and drug delivery [37]. Yet, a fine control over nanofiber population distribution is usually lacking and is so far a limit in obtaining standardized scaffolds. Recently, melt electrospinning writing has emerged as a new technology to overcome this problem. Dalton and his colleagues accurately deposited melt electrospun poly( $\varepsilon$-caprolactone) (PCL) fibers to create complex structures having filament diameters typically in the range of 5 to $30 \mu \mathrm{m}$ [38]. The melt electrospun fibers could consistently be stacked in a manner 
like melt-extrusion based additive manufacturing techniques. More recently, through optimization of spinning parameters (e.g. voltage, flow rate, and spinneret diameters), PCL scaffolds with submicron fiber diameter $(817 \pm 165 \mathrm{~nm})$ were produced using melt electrospinning [39].

\subsection{Surface modification of nanofibrous scaffolds}

Surfaces play a vital role in biology and medicine, as most biological reactions take place at the interface between biological systems [40]. In tissue engineering, the chemical and physical characteristics of the biomaterial surface strongly impact on cell behavior, such as migration, attachment, and proliferation [41]. Although various degradable and nondegradable synthetic polymers have been used as tissue engineering scaffolding materials, a shortcoming of these materials is their lack of biological recognition [6]. Currently, several techniques have been developed to modify the scaffold surface including physical coating and blending, plasma treatment, graft polymerization and wet chemical methods (Figure 2). 

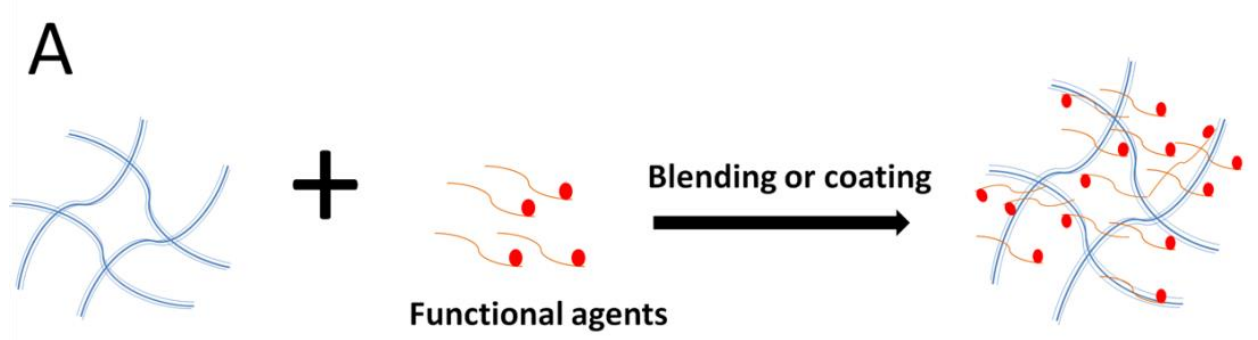

Nanofibers

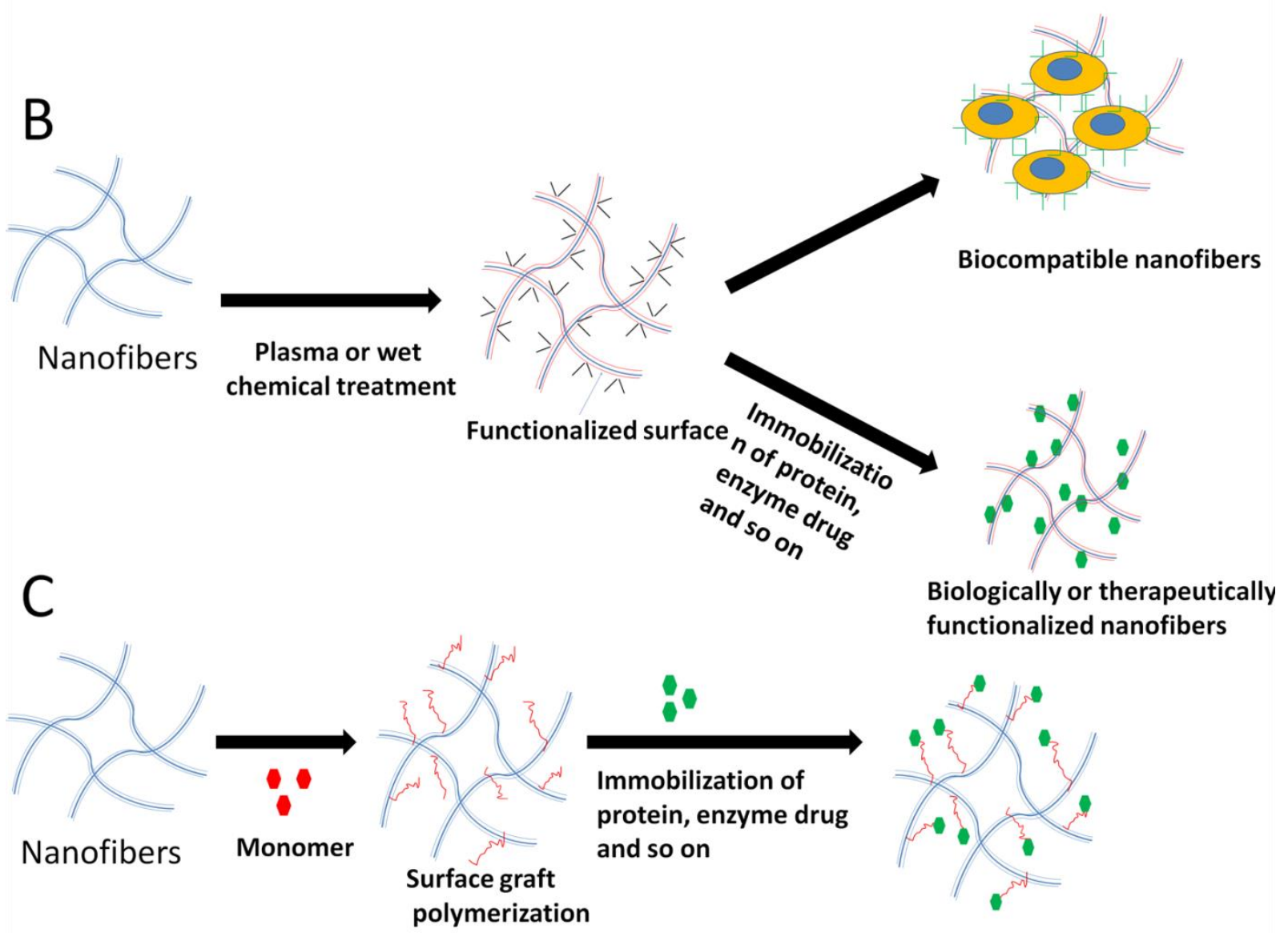

Figure 2. Surface modification techniques of nanofibrous scaffolds. (A) Blending and coating; (B) Plasma or wet chemical treatment; (C) Surface graft polymerization.

\subsubsection{Physical Coating or Blending}

Perhaps the most straightforward and convenient mean of modifying a polymer surface is blending functional molecules and active agents into the bulk polymer or just coating it on the polymer surface. Usually, two or more materials are physically blended together to attain a new biocomposite with superior surface and/or mechanical properties. PLLA nanofibers with hydroxyapatite $(\mathrm{HA})$ particles exposed on their surface were achieved by electrospinning different blended solutions [42, 43]. These composite fibers not only promoted osteoblast adhesion and growth, but also exhibited superior tensile properties as compared to the pure PLLA fibers due to the internal ionic bonding between calcium 
ions in HA particles and ester groups in PLLA [44]. Jun et al. [45] developed electrically conductive blends of Poly (L-lactide-co-caprolactone) (PLCL) and polyaniline (PANi) submicron fibers and investigated their influence on the induction of myoblasts into myotubes. Incorporation of PANi into PLCL fibers significantly increased the conductivity. In addition, the tensile strength and elongation at break of PLCL fibers increased as the concentration of PANi in the fibers decreased, while the Young's modulus exhibited an opposite trend. Silk fibroin/keratin films demonstrated enhanced mechanical properties after blending with gelatin. When further mixed with calcium peroxide, films sustained the release of high level of oxygen over two weeks in vitro, and promoted cellular growth [46]. Taken together, these examples show that blending modifies not only the surface properties of substrate but also their bulk properties.

Coating is another physical approach of functionalizing nanofibrous scaffolds. Although this technique is limited in terms of controllability and coatings maybe prone to detachment from scaffold, it remains one of the easiest and most convenient ways to functionalize biomaterials' surface. In general, a material containing desired functional properties is used to coat nanofibrous scaffolds, aiming at improving their surface biocompatibility and enhancing cell-scaffold interactions. In recent years, coating of functional molecules onto polymers was significantly developed by combining new techniques including coaxial electrospinning and layer-by-layer assembly (LbL). Collagen-coated PCL nanofibers were developed by a coaxial electrospinning technique, with collagen and PCL as inner and outer solutions respectively, and cultured with human dermal fibroblasts [47]. The results demonstrated that collagen-coated PCL nanofibers from coaxial electropinning showed higher efficiency in favoring cell proliferation compared to collagen-coated PCL nanofibers prepared by soaking the PCL matrix in a 10 $\mathrm{mg} / \mathrm{mL}$ collagen solution. The mechanism behind LbL coatings is that positively and negatively charged macromolecules are alternatively introduced onto the substrate surface via strong electrostatic interaction [19]. An environmentally benign surface modification process for poly(ethylene terephthalate) (PET) films was demonstrated by fabricating composite coatings through layer-by-layer assembly with cellulose and chitin nanofibrils [48]. The modified PET films exhibit high light transparency, flexibility, surfacehydrophilicity, and specific nanoporous structures. $\beta$-tricalcium phosphate coating on poly(propylene fumarate) scaffolds showed advantage in terms of osteoconductivity and osteointegration of scaffolds [49]. Aligned and aminolyzed PLA nanofibrous scaffolds, which were coated with graphene oxide, promoted rat Schwann cells growth and 
regulated their orientation[50]. Moreover, this kind of scaffolds induced rat pheochromocytoma 12 cells differentiation and neurite growth in the presence of nerve growth factor. Physical coating could also be used to modulate the scaffolds properties including mechanical property, degradation rate and surface topography. The mechanical strength, degradation rate, and cell behavior of mesoporous bioactive glass could be modulated through controlling the amount of poly(glycerolsebacate) (PGS) coating [51]. Biphasic calcium phosphate scaffolds were coated with PLLA/HA nanocomposites for bone regeneration. Such PLLA/HA coatings improved the compressive strength of scaffolds from 0.31 to $3.56 \mathrm{MPa}$ [52].

\subsubsection{Plasma-based surface modification}

Plasma, also named the forth state of matter, is a gaseous mixture of particles containing charged particles, excited and neutral atoms and molecules, radicals and UV photons [53]. Generally, plasma can be subdivided into two categories according to the temperature of particles: thermal plasma and non-thermal plasma. Non-thermal plasma, which is composed of low temperature particles has been commonly employed to modify the surface of heat-sensitive materials such as biodegradable polymers [54]. By using this technique, diverse functional groups can be effectively incorporated on the target surface of biodegradable polyesters to improve surface adhesion and wetting properties. For example, typical plasma treatment with different gaseous atmospheres such as oxygen, air, and ammonia can introduce carboxyl groups or amine groups to the target surface [55].

After introduction of specific functional groups on the surface of substrates by plasma treatment, synthetic and natural macromolecules could further immobilize monomers (etc. extracellular matrix protein components) on their surface to enhance cellular adhesion and proliferation. This process is called plasma grafting. Nanofibrous scaffolds composed of PLLA and PLGA were fabricated by electrospinning [56]. Their surfaces were treated with oxygen plasma treatment and grafted with hydrophilic acrylic acid. Such surfacemodified scaffolds were shown to improve fibroblast attachment and proliferation in vitro. Plasma polymerization, another plasma-based surface modification approach, is distinct from plasma grafting, since it coats the substrate instead of covalent binding species to a plasma-treated polymer surface [54, 57]. Under plasma condition, gaseous or liquid monomers are converted into reactive fragments, which can in turn recombine to a polymer film deposited onto the substrate exposed to the plasma. To gain more insights on plasma polymerization processes, various $-\mathrm{C}: \mathrm{H}: \mathrm{N}$ thin films were deposited on glass 
under different $\mathrm{NH}_{3} / \mathrm{C}_{2} \mathrm{H}_{4}$ gas ratios, power inputs $\mathrm{W}$, and gas flow rates $\mathrm{F}$ [58]. The results demonstrated that the film growth is determined by the ratio between the reaction parameter $\mathrm{W}$ and $\mathrm{F}$, and the energy dissipated at its surface during the deposition given by ion flux times mean ion energy per deposition rate After treatment with oxygen plasma, PLLA electrospun meshes had an increase in surface roughness as well as surface hydrophilicity [59]. PCL electrospun fibrous scaffolds, which were modified by magnetron discharge plasma during sputtering of a titanium target, increased human endothelial hybrid cell line (EA-hy 926) adhesion and viability within the plasma treatment time of 6 min [60]. However, cell viability would decrease when the plasma treatment increased to 9 min. In another recent study, PCL electrospun scaffolds embedded with PLGA microsphere were treated with cold atmospheric plasma [61]. Human bone marrowderived mesenchymal stem cells showed better cellular growth and total collagen production on this kind of scaffolds compared to the plasma untreated ones.

Plasma treatment, an effective and solvent-free technique, is commonly used for surface modification. The disadvantage of plasma treatment is that only localized surface areas can be treated (in depth from several hundred angstroms to $10 \mathrm{~nm}$ ) without changing the bulk properties of the polymers [41]. Currently, most plasma-based surface modification is performed on two dimensional (2D) polymer substrates, and in some cases on 3D porous scaffold.

\subsubsection{Wet chemical methods}

The cellular response to a biomaterial may be enhanced in synthetic polymer formulations by mimicking the surface roughness created by the associated nanostructured ECM components of natural tissue. PLGA films with nano-structured surface features were developed by treating them in a concentrated $\mathrm{NaOH}$ solution [62]. The results from cell experiments indicated that $\mathrm{NaOH}$ treated PLGA enhanced vascular smooth muscle cell adhesion and proliferation compared to conventional PLGA. This surface modification method is not only used on 2D film surfaces but also on 3D constructs. Chen et al. [63] obtained PCL membranes with nanofibrous topology by coating the membrane surface with electrospun nanofibers. When the nanofibersmodified PCL was treated with $5 \mathrm{M} \mathrm{NaOH}$ for $3 \mathrm{~h}$, a favorable 3T3 fibroblast cells morphology and strong cell attachment were observed on the modified surface, possibly due to the unique hydrophilic surface topography. Electrospun PLLA nanofibrous scaffolds were used as a matrix for mineralization of hydroxyapatite [64]. Carboxylic acid groups were introduced on the surface of PLLA scaffolds by hydrolysis in $\mathrm{NaOH}$ aqueous 
solution. Since calcium ions can bind to the carboxylate groups on the fiber surface, a significant improvement of mineralization was observed on modified PLLA electrospun scaffolds. Hydrophobic polytetrafluoroethylene (PTFE) was wet-chemically treated and subsequently grafted with an endothelial cell specific peptide named arginine-glutamic acid-aspartic acid-valine (REDV). The immobilization of REDV peptide endowed the PTFE substrate a better cellular growth and antifouling properties [65]. Poly(ethylene oxide terephthalate)-poly(butylene terephthalate) rods wre surface-modified by etching chloroform and implanted in the subcutaneous space of pigs to form a fibrocellular tissue capsule. The harvested tissue capsules remodeled towards a vessel after grafting bilaterally as carotid artery interposition graft [66]. PLA electrospun scaffolds were wetchemically treated with polyallylamine to introduce amine groups and subsequently covalently grafted with epidermal growth factor [67]. The functionalized scaffolds supported neural stem-like cells proliferation, which kept viable even in the absence of the soluble growth factor in culture medium.

Although wet chemical methods have been widely applied to modify the surface wettability of biomaterials or to generate new functionalities, some major drawbacks should be noted. This modification technique can cause a partial degradation and scissions of the polymeric chains, resulting in a loss of mechanical properties and a faster degradation process [54]. In addition, the use of hazardous organic solvents might affect cell viability.

\subsubsection{Surface graft polymerization}

Among the surface modification techniques, surface graft polymerization has emerged as a simple, effective and versatile approach in the surface fictionalization of polymers for a wide variety of applications [19]. Grafting exhibits several advantages [19, 68]: Firstly, the ability to modify the polymer surface to possess desired properties through the choice of different monomers. Secondly, the ease and controllable introduction of graft chains with a high density and exact location of graft chains to the surfaces without changing the bulk properties. Finally, a stable chemical bond is formed between the nanofiber surface and the preformed polymer, which offer endurance of the functional component, in contrast to physically coated polymer chains.

Surface graft polymerization is often initiated with plasma discharge, ultraviolet (UV) light, gamma rays, and electron beams [68, 69]. Chua et al. investigated how a functional poly ( $\varepsilon$-caprolactone-co-ethyl ethylene phosphate) (PCLEEP) nanofibrous scaffold with surface-galactose ligand influences rat hepatocytes behavior [70]. The modification of 
nanofibrous scaffolds were achieved by conjugating galactose ligands to a poly (acrylic acid) spacer UV-grafted onto the fiber surface. The functionalized nanofibrous scaffolds exhibited the unique property of promoting hepatocyte aggregates within the mesh and around the fibers. Furthermore, hepatocyte functions are maintained on these functional nanofiber substrates, similar to a galactosylated-film substrate configuration.

The grafting of extra cellular protein like collagen, RGD peptides, and gelatin on nanofiber surfaces has become a popular method to develop biomimicking-tissue scaffolds. For example, the modification of electrospun polyethylene terephthalate (PET) nanofibers involved fiber treatment with formaldehyde to generate hydroxyls on the surface, followed by graft polymerization of methacrylic acid monomers initiated by Cerium (IV). Gelatin was then immobilized on the nanofibers by conjugation to the carboxylic acid moieties on their surface [71]. The grafting of gelatin enhanced the adhesion and spreading of cells on nanofibrous scaffolds. In another study, gelatin was grafted onto PLLA scaffolds via aminolysis of the PLLA fibers following by glutaraldehyde coupling [72].

Despite surface graft polymerization have been established as a universal method for surface modification, some issues arise from its mechanism to initiate grafting. An initiator, which is usually cytotoxic, is involved in grafting initiated by a chemical process. UV light induced grafting may cause polymer degradation, which will compromise the mechanical strength of the scaffold [73]. In addition, grafting induced through high energy irradiation causes scission of the base polymer, which can lead to forming block co-polymer [73, 74].

\subsection{Applications of nanofibrous scaffolds in tissue engineering}

The characteristics of nanofibrous scaffolds that render them promising candidates for tissue engineering include high surface area and porosity, as well as the similarity of their fibrous structure to the physical features of natural ECM [68, 75-77].These features results in a more biologically compatible environment for cells to grow and perform their regular functions. Therefore, nanofibrous scaffolds have been widely used as scaffolds for tissue engineering such as neural, cartilage, vascular, and bone tissue engineering (Table 2). 
Table 2. Nanofibrous scaffold for Tissue Engineering applications

\begin{tabular}{|c|c|c|c|c|}
\hline Materials & $\begin{array}{l}\text { In vitro/In } \\
\text { vivo model }\end{array}$ & Cells & $\begin{array}{l}\text { Potential } \\
\text { application }\end{array}$ & Ref. \\
\hline \multicolumn{5}{|l|}{ (A) Self-assembled nanofiber } \\
\hline $\begin{array}{l}\text { Peptide amphiphile(PA) containing collagen } \\
\text { (PA-collagen) }\end{array}$ & In vitro & $\begin{array}{l}\text { Granule cells } \\
\text { and Purkinje } \\
\text { cells }\end{array}$ & Neural TE & $\begin{array}{l}\text { Sur et al., } \\
2011\end{array}$ \\
\hline $\begin{array}{l}\text { RADA16 (Ac-RADARADARADARADA- } \\
\text { COHN2) }\end{array}$ & In vitro & $\begin{array}{l}\text { neural stem } \\
\text { cells }\end{array}$ & Neural TE & $\begin{array}{l}\text { Gelain et } \\
\text { al., } 2006\end{array}$ \\
\hline RAD16-I peptide & Adult rats & $\begin{array}{l}\text { rat Schwann } \\
\text { cells and } \\
\text { embryonic } \\
\text { NPCs }\end{array}$ & Neural TE & $\begin{array}{l}\text { Guo et al., } \\
2007\end{array}$ \\
\hline PuraMatrix $^{\mathrm{TM}}$ & $\begin{array}{l}\text { Mouse } \\
\text { calvarial bone } \\
\text { defect model }\end{array}$ & & Bone TE & $\begin{array}{l}\text { Misawa et } \\
\text { al., } 2006\end{array}$ \\
\hline $\begin{array}{l}\text { KLD-12 peptide } \\
\text { (AcN-KLDLKLDLKLDL-CNH2) }\end{array}$ & In vitro & $\begin{array}{l}\text { bovine } \\
\text { chondrocytes }\end{array}$ & $\begin{array}{l}\text { Cartilage } \\
\text { TE }\end{array}$ & $\begin{array}{l}\text { Kisiday et } \\
\text { al., } 2002\end{array}$ \\
\hline peptide amphiphile(PA) combining with TGF & $\begin{array}{l}\text { Omentum of } \\
\text { rats }\end{array}$ & $\begin{array}{l}\text { human } \\
\text { mesenchymal } \\
\text { stem cells }\end{array}$ & $\begin{array}{l}\text { Cartilage } \\
\text { TE }\end{array}$ & $\begin{array}{l}\text { Shah et al., } \\
2010 \text { ) }\end{array}$ \\
\hline \multicolumn{5}{|l|}{ (B) Phase separation nanofiber } \\
\hline Tyrosine-derived polycarbonate (TyrPC) & $\begin{array}{l}\text { rabbit } \\
\text { calvarial } \\
\text { critical-sized } \\
\text { defect }\end{array}$ & - & Bone TE & $\begin{array}{l}\text { Kim et al., } \\
2012\end{array}$ \\
\hline Chitosan/poly( $\varepsilon$-caprolactone $)$ blend & In vitro & $\begin{array}{l}\text { Bovine } \\
\text { chondrocyte }\end{array}$ & $\begin{array}{l}\text { Cartilage } \\
\text { TE }\end{array}$ & $\begin{array}{l}\text { Neves et al., } \\
2011\end{array}$ \\
\hline Poly-L-lactide (PLLA) & In vitro & $\begin{array}{l}\text { Human aortic } \\
\text { smooth muscle } \\
\text { cells } \\
\text { (HASMCs) }\end{array}$ & Vascular TE & $\begin{array}{l}\mathrm{Hu} \text { et al., } \\
2010\end{array}$ \\
\hline \multicolumn{5}{|l|}{ (C) Electrospinning nanofiber } \\
\hline Poly(l-lactic acid) (PLLA) & In vitro & $\begin{array}{l}\text { neural stem } \\
\text { cells (NSCs) }\end{array}$ & Neural TE & $\begin{array}{l}\text { Yang et al., } \\
2005\end{array}$ \\
\hline $\operatorname{Poly}(\varepsilon$-caprolactone) (PCL) & In vitro & $\begin{array}{l}\text { human } \\
\text { mesenchymal } \\
\text { stem cells } \\
\text { (hMSCs) }\end{array}$ & $\begin{array}{l}\text { Cartilage } \\
\text { TE }\end{array}$ & $\begin{array}{l}\mathrm{Li} \text { et al., } \\
2005\end{array}$ \\
\hline $\begin{array}{l}\text { Poly(L-lactic-co- } \varepsilon \text {-caprolactone) }[\text { P(LLA-CL) }] \\
(75: 25)\end{array}$ & In vitro & $\begin{array}{l}\text { smooth muscle } \\
\text { cell }\end{array}$ & Vascular TE & $\begin{array}{l}\text { Mo and } \\
\text { Weber, } \\
2004\end{array}$ \\
\hline Gelatin-modified PET nanofibers & In vitro & $\begin{array}{l}\text { endothelial } \\
\text { cells }\end{array}$ & Vascular TE & $\begin{array}{l}\text { Ma et al., } \\
200\end{array}$ \\
\hline PCL-collagen & $\begin{array}{l}\text { Rabbit aortal- } \\
\text { iliac bypass } \\
\text { model }\end{array}$ & vascular cells & Vascular TE & $\begin{array}{l}\text { Tillman et } \\
\text { al., } 2009\end{array}$ \\
\hline $\operatorname{Poly}(\varepsilon$-caprolactone) (PCL) & In vitro & $\begin{array}{l}\text { Rabbit } \\
\text { mesenchymal } \\
\text { stem cells } \\
\text { (MSCs) }\end{array}$ & Bone TE & $\begin{array}{l}\text { Yoshimoto } \\
\text { et al., } 2003\end{array}$ \\
\hline $\operatorname{Poly}(\varepsilon$-caprolactone) (PCL) & $\begin{array}{l}\text { The omentum } \\
\text { of rats }\end{array}$ & $\begin{array}{l}\text { Rats } \\
\text { mesenchymal } \\
\text { stem cells } \\
\text { (MSCs) }\end{array}$ & Bone TE & $\begin{array}{l}\text { Shin et al., } \\
2004\end{array}$ \\
\hline
\end{tabular}




\subsubsection{Nanofibrous scaffolds for neural tissue engineering}

The nervous system coordinates the action of human and transmits signals between different parts of the body. However, the nervous system has a limited capacity to repair itself after an injury. As a result, patients who have injures or traumas in the nervous system often suffer from the loss of sensory or motor function, and neuropathic pains [25, 78]. In order to facilitate the re-growth of nerves, many therapeutic approaches have been attempted. One of the most promising approaches is to adopt neural tissue engineering strategy that employs a scaffold or conduit to facilitate the nerve regeneration.

Yang et al. [79] studied aligned and random PLLA electrospun nanofibrous scaffolds for neural tissue engineering. Their result indicated that neural stem cell (NSCs) elongated and their neurites out grew along the direction of the fiber orientation of the aligned nanofibers (Figure 3). Furthermore, NSCs on aligned nanofibrous scaffolds showed higher rate of differentiation than on microfibers. Thus, the aligned PLLA nanofibrous scaffolds showed potential application in neural tissue engineering. ArginineAlanine-Aspartate (RAD)16-I are the most commonly used peptides in self-assembled peptide scaffolds for neural cell culture [80-82]. Semino et al. [81] developed RAD16-I self-assembled peptide scaffolds as a 3D culture system for cell encapsulation. Primary rat hippocampal cells were cultured on top of the self-assembled nanofibrous scaffold. Immunochemistry assays showed that glial cells and neurons increasingly migrated into the peptide scaffolds to an approximate depth of $400-500 \mu \mathrm{m}$ from the edge of the tissue after 1 week of culture. Furthermore, mitotic activity of neural cells was maintained for 3 days after migration, which was attributed to the presence of the nanofibrous scaffold environment resembling the native ECM. These results revealed that self-assemble peptide nanofibrous scaffolds are potential substrate for supporting neural tissue regeneration. A hybrid nanofibrous matrix with homogeneous fiber diameter of 20-30 nm was designed by self-assembly of PA with the ability of presenting laminin epitopes (IKVAV or YIGSR) and collagen molecules [83]. Granule cells and Purkinje cells, two major neuronal subtypes of the cerebellar cortex, were cultured on the hybrid scaffold. The result showed the ability to modulate neuron survival and maturation by easy manipulation of epitope density. 

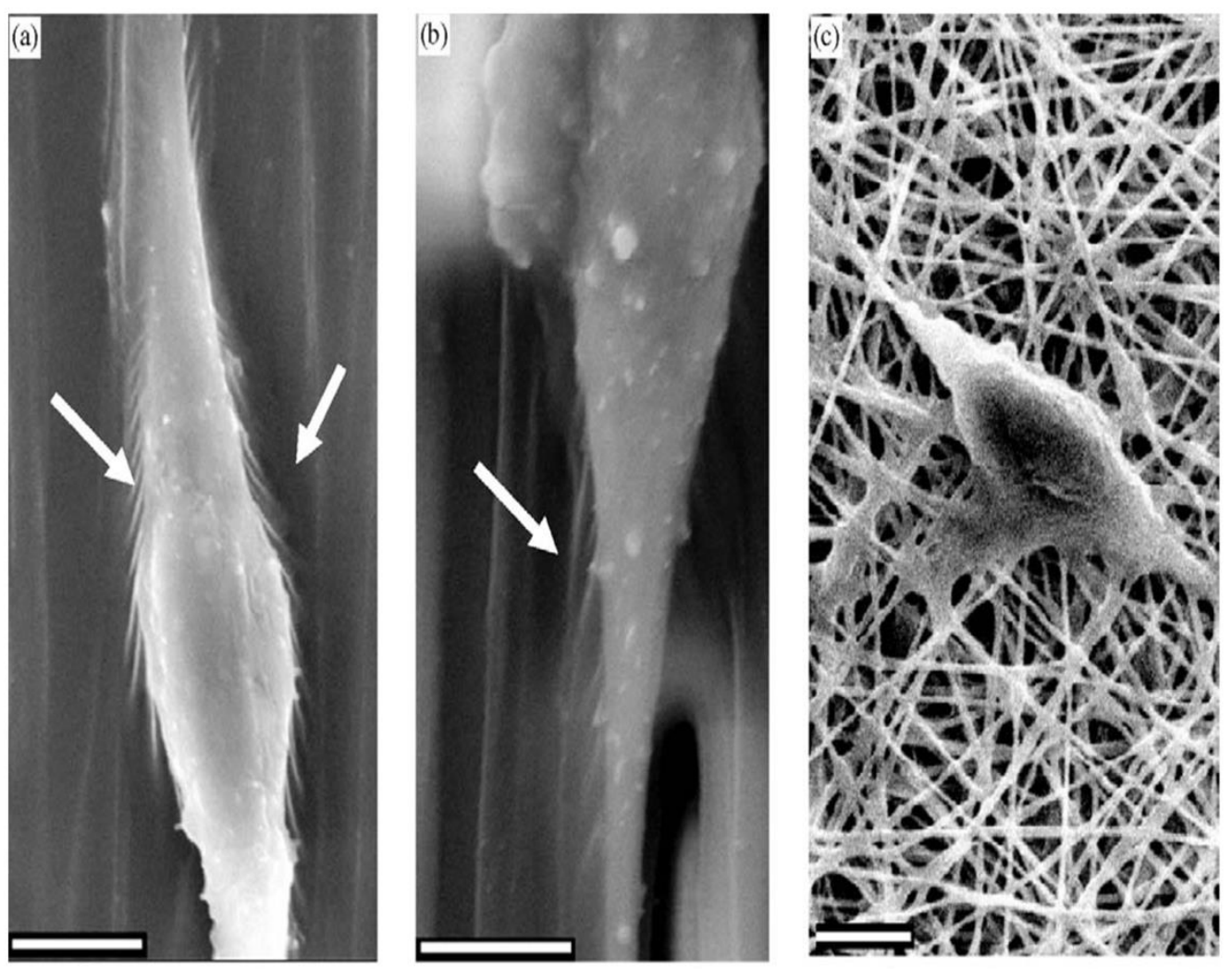

Figure 3. SEM micrographs of NSCs seeded on (a) nano scale aligned fibers; (b) micro scale aligned fibers and; (c) nano scale ramdom fibers for 2 days showing the cell-matrix adhesion between the NSCs and the PLLA fibers Bar $=5 \mathrm{~mm}$ (Yang et al. 2005).

In addition to in vitro experiments, self-assembled nanofibrous scaffolds have been transplanted into animal models for the treatment of central nervous system injuries. Selfassembled RAD16-I peptide scaffold combined with adult rat Schwann cells and embryonic NPCs were transplanted into adult rats [15]. The results indicated that the scaffolds integrated well with the host tissue with no obvious gap between the implants and the injured sites. A noteworthy observation from these experiments was that large amount of host cells migrated into the scaffolds and extensive blood vessel formation was observed in the implants. Functionalized self-assembling peptides were incorporated into electrospun scaffolds via coaxial electrospinning [84]. The scaffold was fabricated from an emulsion of PCL/PLGA composite together with a functionalized self-assembling peptide as a shell, while PCL/PLGA composite as core demonstrated a better cell viability of mice neural stem cells in vitro and in vivo inflammatory response in a rodent spinal cord injury model. Silk-gold based artificial conduits, which were fabricated by electrospinning, promoted cellular adhesion and proliferation of Schwann cells in vitro [85]. Moreover, the rat sciatic nerve injury model demonstrated that the artificial conduits did not evoke any toxic or immunogenic responses. 


\subsubsection{Nanofibrous scaffolds for cartilage tissue engineering}

Cartilage, a predominantly avascular, alymphatic, and aneural tissue, is composed of chondroblasts embedded within a dense extracellular matrix (ECM) [86]. Cartilage is classified in three types: elastic cartilage, hyaline cartilage and fibrocartilage. Cartilage damage resulting from developmental abnormalities, trauma, aging related degeneration and joint injury cause disability and extensive pain [78, 87]. Due to its limited capacity to self-regenerate, cartilage is an ideal candidate for tissue engineering. In cartilage tissue engineering, chondrocytes and mesenchymal stem cells (MSCs) are commonly used for cartilage repair. Electrospun PCL nanofibrous scaffolds were seeded with human bone marrow-derived MSCs to investigate their ability to support in vitro MSCs chondrogenesis [88]. The results demonstrated that $P C L$ nanofibrous scaffolds in the presence of transforming growth factor- $\beta 1$ (TGF- $\beta 1$ ) induced the differentiation of MSCs to chondrocytes comparable to pellet cultures. Although no inductive property of PCL nanofibrous scaffolds was observed, these meshes had better mechanical properties than cell pellets making them suitable for cartilage tissue engineering. Kisiday et al. [89] investigated a self-assembling peptide hydrogel scaffold for cartilage regeneration. They combined the peptide KDK-12 with bovine chondrocytes and allowed to self-assemble into a hydrogel. Their results indicated that chodrocytes proliferated and maintained a chondrocytic phenotype in the hydrogel. Cells were able to produce cartilage-like ECM which was rich in type-Il collagen and proteoglycans. PAs synthesized with a peptide binding sequence to TGF- $\beta 1$ were design to form nanofibers via self-assembling for cartilage regeneration [17]. The study demonstrated that these scaffolds support the survival and promote the chondrogenic differentiation of human MSCs. In vivo experiments showed that these scaffolds promoted regeneration of articular cartilage in a rabbit model with or even in the absence of exogenous growth factor (Figure 4). 

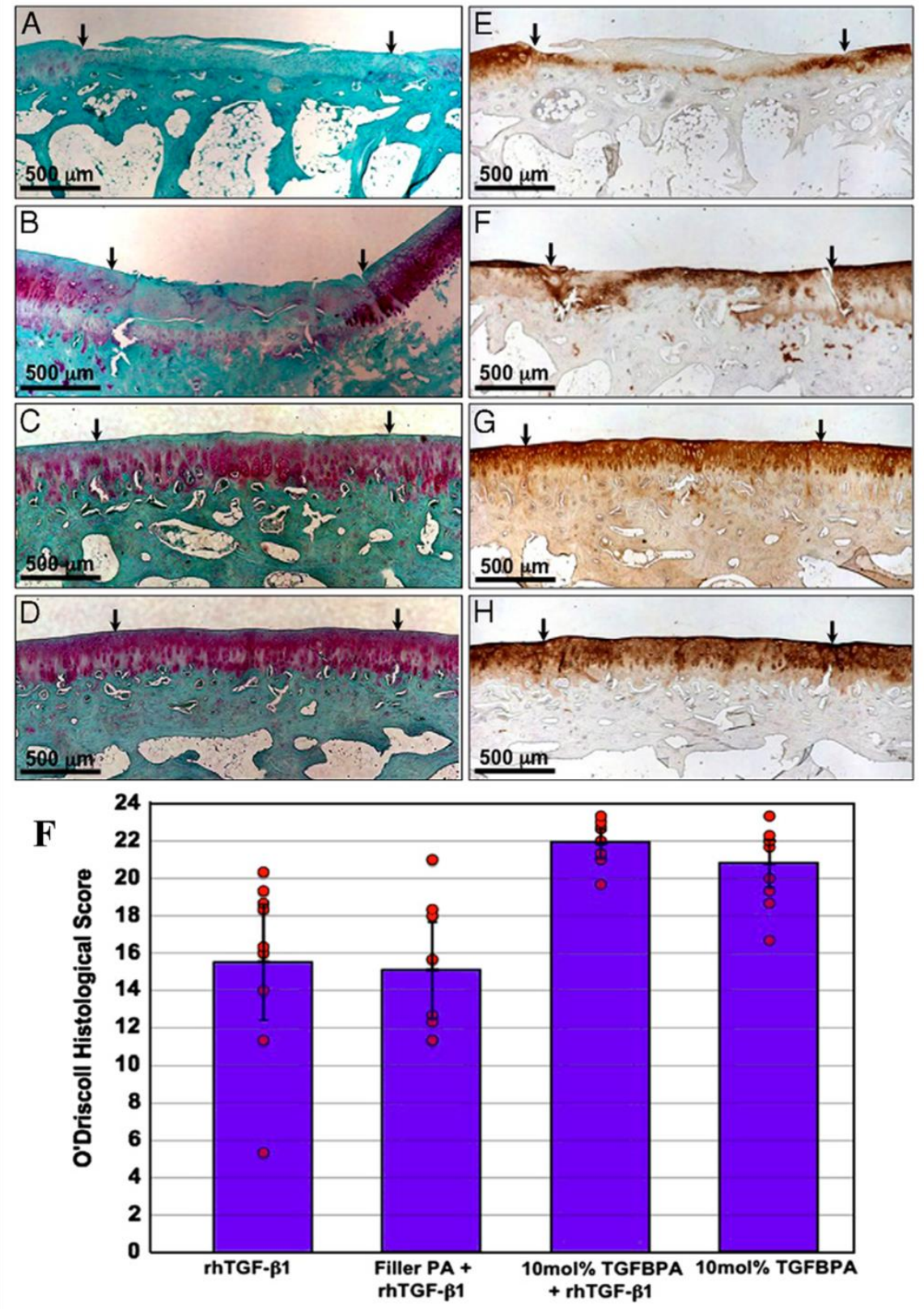

Figure 4. (A-H) Histological evaluation of sample sections 12 wks after treatment. Safranin-O staining for glycosaminoglycans $(A-D)$ and type II collagen staining $(E-H)$ in articular cartilage defects treated with (A, E) $100 \mathrm{ng} / \mathrm{mL}$ TGF- $\beta 1$ (100TGF), (B, F) filler PA+ TGF- $\beta 1,(C, G) 10 \%$ TGFBPA+ TGF- $\beta 1$, and (D, H) $10 \%$ TGFBPA alone 12 weeks postop; (F) Histological scores of 12 week in vivo samples. Circles represent scores for individual specimens in each group $(n=8-10)$ [17].

\subsubsection{Nanofibrous scaffolds for vascular tissue engineering}

Blood vessels perform a very important function of carrying and transporting blood to and from the heart. In addition, they are also important places for the exchange of water and other chemicals between blood and the tissue [19, 37]. Mo et al. [90] developed an aligned electrospun nanofibrous scaffold from biodegradable PLLA-CL $(75: 25)$ with the goal of developing constructs for vascular tissue engineering. The fabricated nanometric 
fibers resembled the dimensions of natural ECM, possessed mechanical properties comparable to human coronary artery, and supported smooth muscle cell adhesion and proliferation.

A major reason for graft failing is that the graft surface is only partially covered by endothelial cells following a degenerative state. To overcome this problem, developed PET nanofibrous by electrospinning and modified its surface by grafting gelatin. Their study indicated that gelatin-modified PET nanofibers were favorable for spreading and proliferation of endothelial cells and maintained cell phenotype [71]. Tillman et al. [91] studied the in vivo stability of electrospun PCL-collagen scaffolds in vascular regeneration in a rabbit aortal-iliac bypass model. Their finding suggested that PCL-collagen scaffolds maintained enough mechanical strength for cell growth and other physiologic conditions.

\subsubsection{Nanofibrous scaffolds for bone tissue engineering}

Bone is a composite material made from an organic phase of collagen and glycoproteins and an inorganic phase primarily consisting of hydroxyapatite (HA) [92]. The organic phase provides bone resilient nature and the inorganic minerals is responsible for bone hardness [19, 93]. Bone may be lost caused by trauma, fractures, periodontitis, cancer, infectious disease, and osteoporosis [94]. Presently, many approaches have been developed for bone regeneration, such as autografts, metal implants, and allografts. However, all these methods have obvious drawbacks. For example, autografts present problems associated with a limited donor source as well as a secondary surgery site. Therefore, tissue engineering approaches are presently being investigated as a promising method for bone regeneration. Yoshimoto et al. [95] studied the potential of non-woven PCL scaffolds generated by electrospinning for bone tissue engineering. MSCs derived from bone marrow of neonatal rats were seeded on the nanofibers. The results demonstrated that MSCs migrated into the scaffolds and produced abundant ECM. Based on this study, Shin et al. [96] implanted MSCs along with PCL nanofibrous scaffolds into the omentum of rats. Their study indicated ECM formation throughout the scaffolds along with the evidence of mineralization and type I collagen synthesis. When $\mathrm{HA}$ is incorporated into nanofibrous scaffolds it does not only improve the mechanical properties, but also creates more biomimetic constructs. Either nanoparticles of HA, morphogenetic proteins (e.g. BMP-2) or both were incorporated in electrospun nanofibrous scaffolds of silk fibroin [97]. Scaffolds were seeded with human MSCs for 31 days in osteogenic medium. HA nanoparticels were associated with improved bone formation. Furthermore, when silk fibroin scaffolds were combined with both nanoparticles 
of $\mathrm{HA}$ and BMP-2, they were associated with the highest observed mineralization.

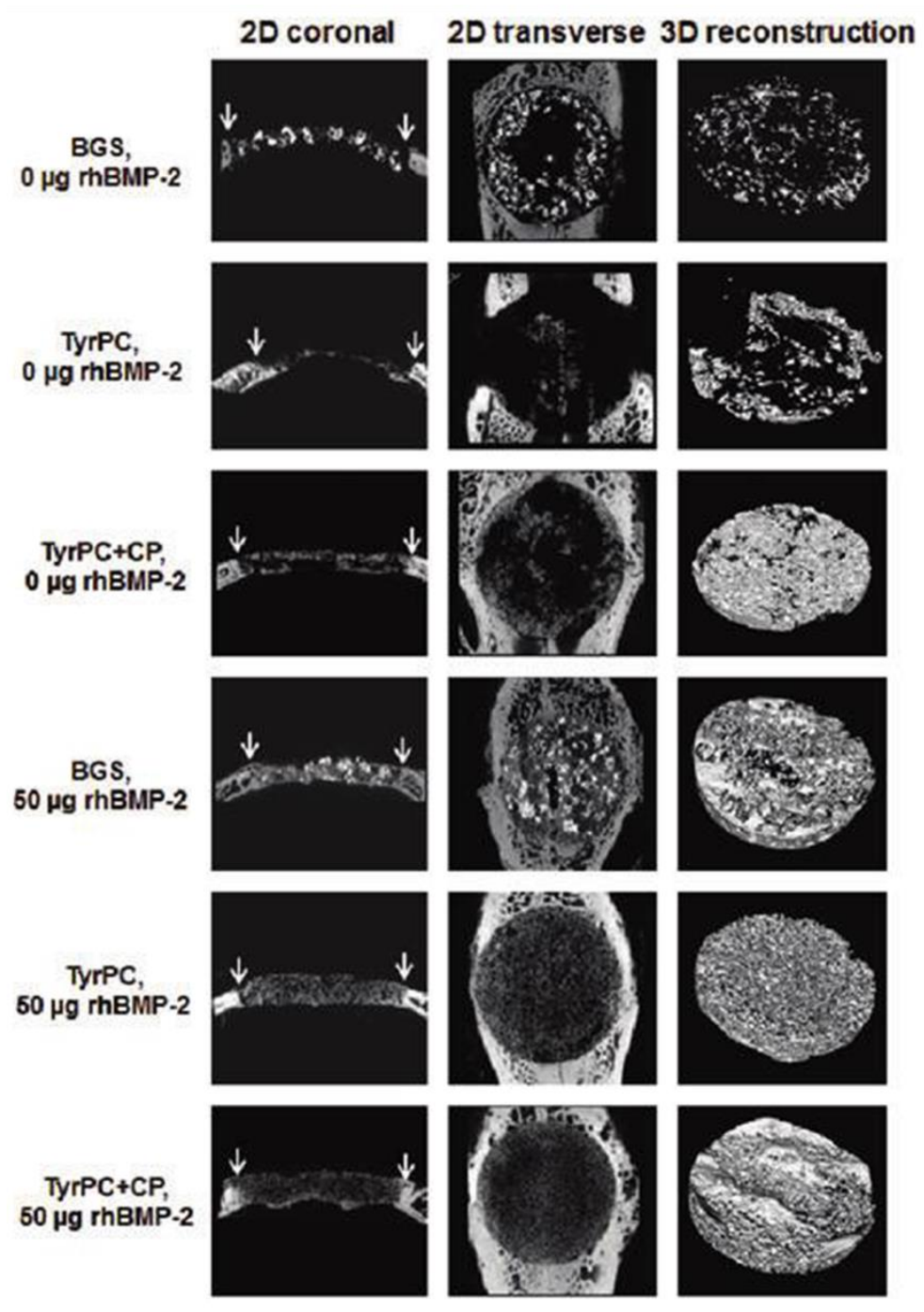

Figure 5. Representative microcomputed tomography images of bone regeneration in the rabbit model at 6 weeks postimplantation. White arrows in the first column identify the defect site. Remaining b-tricalcium phosphate fragments are identified in the $2 \mathrm{D}$ transverse images of the bone graft substitute (BGS) scaffolds [98].

Porous three-dimensional tyrosine-derived polycarbonate (TyrPC) scaffolds were developed for bone regeneration using a combination of salt-leaching and phase separation techniques [98]. The TyrPC scaffolds treated with or without recombinant human bone morphogenetic protein-2 (rhBMP-2) were implanted in a rabbit calvarial critical-sized defect model for 6 weeks. The in vivo studies showed that TyrPC scaffolds treated with rhBMP-2 or coated with calcium phosphate alone promoted bone regeneration at 6 weeks post-implantation (Figure 5). Moreover, TyrPC polymeric scaffolds even without addition any biological agents induced similar bone regeneration to a commercially available bone graft substitute. Biomimetic nanofibrous scaffolds made 
of hydroxyapatite/collagen/chitosan (HAp/Col/CTS) showed higher capacity than control scaffolds (tissue culture plate, CTS, and HAp/CTS) for the osteogenesis of induced pluripotent stem cell-derived mesenchymal stem cells (iPSC-MSCs) in vitro [99]. Furthermore, mouse cranial defects were created to investigating the efficacy of biomimetic scaffolds for bone repair in vivo. After six weeks implantation, HAp/Col/CTS scaffolds showed nearly a 2-fold higher bone mineral density than other scaffolds. Mimicking the formation of vertebrate bone during early stage, Rajamani et al. developed nanofibrous collagen scaffolds containing catecholamines and $\mathrm{Ca}^{2+}$ for bone regeneration [100]. The developed collagen composite scaffolds had outstanding mechanical properties with a Young's modulus close to cancellous bone. Human fetal osteoblasts displayed increased cell proliferation and differentiation in the collagen composite scaffolds compared to tissue culture plate or pristine collagen mats.

\subsection{Scope and Outline of Thesis}

Mimicking the architecture of ECM is one of the key challenges of tissue engineering. Amongst all the methods used to generate artificial ECM, nanofibrous scaffolds demonstrated the most promising results. Nanofibrous scaffolds, irrespective of their method of synthesis, are characterized by high surface area and enhanced porosity, which are highly desired for tissue engineering and drug delivery applications.

At present, there are three dominant approaches to generate nanofibrous scaffolds: molecular self-assembly, phase separation, and electrospinning. Among these approaches, electrospinning is the most widely studied approach since it is a simple and versatile technique that can produce fibers with a diameter from the micrometer down to nanometer range. Furthermore, it allows one to tailor many aspects of the resulting scaffolds [29, 101, 102]: (1) the fiber diameter can easily be controlled by varying the solution properties and the processing parameters; (2) the nanofibers can be collected with a rich variety of aligned/random structures using specially designed collectors; (3) they can be stacked and/or folded to form complex structures or architectures; (4) they can be obtained with a hollow or core-sheath structure by changing the configuration of the spinneret; (5) nanofibers with porous surface structure can be achieved by altering the parameters such as solvent and humidity. The potential of nanofibrous scaffolds for the regeneration of various tissues are now under extensive investigation. In spite of the great achievements behind the designing of nanofibrous scaffolds via electrospining, there is still plenty of room for improvement. The further development of meltelectrospinning direct writing in the last few years has shown the advantage of creating 
nanofiber patterns that can guide tissue organization. In fabricating smart scaffolds to direct cell fate, more knowledge over chemical and physical stimuli that can be built-in the biomaterial formulation of choice is desirable. In developing new techniques to fabricate smart nanofibrous scaffolds, the configuration seen in native tissues should be taken into consideration if we want to achieve the highest degree of biomimicry possible. Finally, charge repulsion should be improved when aiming at clinically relevant large scaffold fabrication.

To better restore and recover the damaged tissues or organs, there is a great need for bioactive scaffolds which not only provide space and mechanical properties for cellular growth and migration, but can also offer chemical and physical cues to control cell fate and finally direct tissue formation. The work presented hereafter is aimed to provide a small library of possible strategies for designing bioactive scaffolds for skeletal tissue engineering through electrospuning-based techniques.

Human mesenchymal stem/stromal cells (hMSCs) have been chosen as a cell model, because of their known potential as a cell source for regenerative medicine due to their capacity to differentiate into a variety of cell type, including adipocytes, chondrocytes, and osteoblasts[103]. The approaches to achieve this goal are developed as follows: (1) investigate the effect of chemical composition of a scaffold and correlate its degradation and wettability properties to the response of seeded cells; (2) fabricate novel flexible ceramic fibrous mats with tailored surface roughness and study the influence of surface roughness of electrospun scaffolds on cell faith; (3) modify the surface roughness of electrospun fibers via regulating humidity during electrospinning; (4) fabricate buckled fibrous meshes to mimic the crimped feature of collagen fibers in native tissue using thermal shrinkable films; (5) and develop a scaffold mimicking the zonal organization of articular cartilage using a new direct writing electrospun technique.

Scaffold-based tissue engineering is nowadays an established strategy for repairing damaged tissues and organs. Therefore, there is a great need for new biodegradable polymers, like polyesters, to fabricate scaffolds with enhanced bioactivity Such new bioactive scaffolds should be characterized by having more flexibility in tuning their physical (e.g. mechanical and hydrolysis properties) and biological (e.g. bioactive cues for triggering stem cell differentiation) properties. When achieved, this will give the unprecedented opportunity to steer cell differentiation through the rationale design of synthetic functional materials, whereas today this is mostly done by biological molecules in solution or coupled to synthetic materials. To this end, in chapter 2 electrospun 
scaffolds were fabricated from a new copolyester family containing either butylene thiodiglycolate (BTDG) or butylene diglycolate (BDG) sequences by reactive blending. The synthesized polyesters displayed tunable mechanical properties and hydrolysis rate depending on the molecular architecture and on the kind of etheroatom introduced along the polymer backbone. The potential of these new scaffolds in skeletal tissue engineering was examined in vitro by culturing hMSCs on them in basic or differentiation media.

The main drawback in hard tissue regeneration of bioactive ceramic scaffolds is their low mechanical strength. Stabilized zirconia, especially the tetragonal $3 \%$ yttriumstabilized zirconia (YSZ) phase, has been considered as a bioinert ceramic material with high mechanical strength. In chapter 3 , novel flexible nanofibrous YSZ scaffolds were fabricated by electrospinning. Their surface roughness could be tailored by varying the heat treatment methods. The performence of this new ceramic scaffold in bone tissue engineering was further investigated. It has been fully demonstrated that the surface roughness of 2D materials have influence on cellular response, such as cell proliferation, attachment, and differentiation. However, the biological environment for cells in a tissue or organ is a 3D structure instead of a 2D flat substrate. To investigate the effect of surface roughness of flexible ceramic 3D nanofibrous scaffolds on the skeletal differentiation of hMSCs, electrospun meshes with same surface chemistry, yet different surface roughness, were obtained by varying the relative humidity levels during electrospinning (Chapter 4).

The periodic waviness of native collagen fibrils is observed in nearly all human tissue, such as tendon, blood vessels, and valve leaflets. The features of such a waived or crimped structure ensure that loads are sustained both by the surrounding matrix and the fiber network at low levels of tensile strain. To get a better resemblance of natural collagen fibrils, in chapter 5 , we exploit a simple but efficient and versatile method to produce crimp patterned electrospun fibers by using thermally shrinkable films. hMSCs were cultured on the wavy fibers to evaluate whether cell infiltration into the scaffolds could be also improved, which is nowadays still one of the major drawback of electrospun meshes. Given the resemblance of the obtained fibers with the crimp patterns typical of tendons and ligaments, we also investigated if the wavy patterns could regulate the TGF$\beta$ pathway, a key signaling pathway involved in tendon and ligament maintenance.

One of the limitations of traditional solution electrospinning technique lies in designing of scaffold's architecture, which often results in a densely packed structure. However, the structure of most human tissue or organ is a more complex hierarchical structure. For 
example, articular cartilage contains four main zones: the superficial, middle, deep and calcified cartilage zone. To overcome this problem, in chapter 6 , a new technique for the fabrication of microfibrous structures in a precise and controllable way was developed, using a direct-write electrospinning process. Furthermore, a scaffold mimicking the zonal organization of articular cartilage was produced by this method and its performance on cartilage tissue formation was evaluated through the chondrogenic differentiation of seeded hMSCs in vitro.

Chapter 7 gives an overview of the obtained results and of future perspective in designing scaffold for skeletal tissue engineering.

Finally, chapter 8 discuss the valorisation potential of the research presented in this thesis. 


\section{References}

[1] T. Subbiah, G. Bhat, R. Tock, S. Parameswaran, S. Ramkumar, Electrospinning of nanofibers, Journal of Applied Polymer Science 96(2) (2005) 557-569.

[2] S. Watari, K. Hayashi, J.A. Wood, P. Russell, P.F. Nealey, C.J. Murphy, D.C. Genetos, Modulation of osteogenic differentiation in hMSCs cells by submicron topographically-patterned ridges and grooves, Biomaterials (2011).

[3] M. Goldberg, R. Langer, X. Jia, Nanostructured materials for applications in drug delivery and tissue engineering, Journal of biomaterials science. Polymer edition 18(3) (2007) 241.

[4] D. Philp, J.F. Stoddart, Self-assembly in natural and unnatural systems, Angewandte Chemie International Edition in English 35(11) (1996) 1154-1196.

[5] J.D. Hartgerink, E. Beniash, S.I. Stupp, Self-assembly and mineralization of peptide-amphiphile nanofibers, Science 294(5547) (2001) 1684-1688.

[6] L.A. Smith, X. Liu, P.X. Ma, Tissue engineering with nano-fibrous scaffolds, Soft matter 4(11) (2008) 2144-2149.

[7] H. Cui, M.J. Webber, S.I. Stupp, Self-assembly of peptide amphiphiles: From molecules to nanostructures to biomaterials, Peptide Science 94(1) (2010) 1-18.

[8] A.M. Hung, S.I. Stupp, Simultaneous self-assembly, orientation, and patterning of peptide-amphiphile nanofibers by soft lithography, Nano letters 7(5) (2007) 1165-1171.

[9] H. Cui, T. Muraoka, A.G. Cheetham, S.I. Stupp, Self-assembly of giant peptide nanobelts, Nano letters 9(3) (2009) 945-951.

[10] Y. Zhao, M. Tanaka, T. Kinoshita, M. Higuchi, T. Tan, Nanofibrous scaffold from self-assembly of [beta]-sheet peptides containing phenylalanine for controlled release, Journal of Controlled Release 142(3) (2010) 354-360.

[11] C. Zhong, A. Cooper, A. Kapetanovic, Z. Fang, M. Zhang, M. Rolandi, A facile bottom-up route to selfassembled biogenic chitin nanofibers, Soft matter 6(21) (2010) 5298-5301.

[12] B. Sarkar, L.E. O'Leary, J.D. Hartgerink, Self-assembly of fiber-forming collagen mimetic peptides controlled by triple-helical nucleation, Journal of the American Chemical Society 136(41) (2014) 1441714424.

[13] K.-H. Shin, J.-W. Kim, Y.-H. Koh, H.-E. Kim, Novel self-assembly-induced 3D plotting for macro/nanoporous collagen scaffolds comprised of nanofibrous collagen filaments, Materials Letters 143 (2015) 265268.

[14] Y. Loo, A. Lakshmanan, M. Ni, L.L. Toh, S. Wang, C.A. Hauser, Peptide bioink: self-assembling nanofibrous scaffolds for three-dimensional organotypic cultures, Nano letters 15(10) (2015) 6919-6925.

[15] J. Guo, H. Su, Y. Zeng, Y.X. Liang, W.M. Wong, R.G. Ellis-Behnke, K.F. So, W. Wu, Reknitting the injured spinal cord by self-assembling peptide nanofiber scaffold, Nanomedicine: Nanotechnology, Biology and Medicine 3(4) (2007) 311-321.

[16] T.D. Sargeant, M.O. Guler, S.M. Oppenheimer, A. Mata, R.L. Satcher, D.C. Dunand, S.I. Stupp, Hybrid bone implants: self-assembly of peptide amphiphile nanofibers within porous titanium, Biomaterials 29(2) (2008) 161-171. 
[17] R.N. Shah, N.A. Shah, M.M.D.R. Lim, C. Hsieh, G. Nuber, S.I. Stupp, Supramolecular design of selfassembling nanofibers for cartilage regeneration, Proceedings of the National Academy of Sciences 107(8) (2010) 3293-3298.

[18] I. Smith, X. Liu, L. Smith, P. Ma, Nanostructured polymer scaffolds for tissue engineering and regenerative medicine, Wiley Interdisciplinary Reviews: Nanomedicine and Nanobiotechnology 1(2) (2009) 226-236.

[19] S. Ramakrishna, An introduction to electrospinning and nanofibers, World Scientific Pub Co Inc2005.

[20] Z. Zhang, J. Hu, P.X. Ma, Nanofiber-based delivery of bioactive agents and stem cells to bone sites, Advanced drug delivery reviews (2012).

[21] J.M. Holzwarth, P.X. Ma, 3D nanofibrous scaffolds for tissue engineering, J. Mater. Chem. 21(28) (2011) 10243-10251.

[22] J. Mao, S. Duan, A. Song, Q. Cai, X. Deng, X. Yang, Macroporous and Nanofribrous Poly (Lactide-coGlycolide)(50/50) Scaffolds Via Phase Separation Combined with Particle-Leaching, Materials Science and Engineering: C (2012).

[23] K.M. Woo, V.J. Chen, P.X. Ma, Nano-fibrous scaffolding architecture selectively enhances protein adsorption contributing to cell attachment, Journal of Biomedical Materials Research Part A 67(2) (2003) 531-537.

[24] Z. Zhang, M.J. Gupte, X. Jin, P.X. Ma, Injectable Peptide Decorated Functional Nanofibrous Hollow Microspheres to Direct Stem Cell Differentiation and Tissue Regeneration, Advanced Functional Materials 25(3) (2015) 350-360.

[25] M. Prabaharan, R. Jayakumar, S. Nair, Electrospun Nanofibrous Scaffolds-Current Status and Prospects in Drug Delivery, Adv Polym Sci (2011) 1-22.

[26] T.J. Sill, H.A. Von Recum, Electrospinning: applications in drug delivery and tissue engineering, Biomaterials 29(13) (2008) 1989-2006.

[27] J. Deitzel, W. Kosik, S. McKnight, N. Beck Tan, J. DeSimone, S. Crette, Electrospinning of polymer nanofibers with specific surface chemistry, Polymer 43(3) (2002) 1025-1029.

[28] K. Lee, H. Kim, M. Khil, Y. Ra, D. Lee, Characterization of nano-structured poly ( $\varepsilon$-caprolactone) nonwoven mats via electrospinning, Polymer 44(4) (2003) 1287-1294.

[29] W. Liu, S. Thomopoulos, Y. Xia, Electrospun Nanofibers for Regenerative Medicine, Advanced Healthcare Materials (2011).

[30] D. Li, Y. Wang, Y. Xia, Electrospinning nanofibers as uniaxially aligned arrays and layer-by-layer stacked films, Advanced Materials 16(4) (2004) 361-366.

[31] J. Stitzel, J. Liu, S.J. Lee, M. Komura, J. Berry, S. Soker, G. Lim, M. Van Dyke, R. Czerw, J.J. Yoo, Controlled fabrication of a biological vascular substitute, Biomaterials 27(7) (2006) 1088-1094.

[32] C.L. He, Z.M. Huang, X.J. Han, L. Liu, H.S. Zhang, L.S. Chen, Coaxial electrospun poly (L-lactic acid) ultrafine fibers for sustained drug delivery, Journal of Macromolecular Science Part B--Physics 45(4) (2006) 515-524.

[33] Y. Zhang, X. Wang, Y. Feng, J. Li, C. Lim, S. Ramakrishna, Coaxial electrospinning of (fluorescein isothiocyanate-conjugated bovine serum albumin)-encapsulated poly ( $\varepsilon$-caprolactone) nanofibers for sustained release, Biomacromolecules 7(4) (2006) 1049-1057. 
[34] J. Venugopal, S. Low, A.T. Choon, S. Ramakrishna, Interaction of cells and nanofiber scaffolds in tissue engineering, Journal of Biomedical Materials Research Part B: Applied Biomaterials 84(1) (2008) 3448.

[35] M. Wang, N. Jing, C.B. Su, J. Kameoka, C.K. Chou, M.C. Hung, K.A. Chang, Electrospinning of silica nanochannels for single molecule detection, Applied physics letters 88 (2006) 033106.

[36] D. Li, Y. Xia, Direct fabrication of composite and ceramic hollow nanofibers by electrospinning, Nano letters 4(5) (2004) 933-938.

[37] W. Cui, Y. Zhou, J. Chang, Electrospun nanofibrous materials for tissue engineering and drug delivery, Science and Technology of Advanced Materials 11 (2010) 014108.

[38] T.D. Brown, P.D. Dalton, D.W. Hutmacher, Direct writing by way of melt electrospinning, Advanced Materials 23(47) (2011) 5651-5657.

[39] G. Hochleitner, T. Jüngst, T.D. Brown, K. Hahn, C. Moseke, F. Jakob, P.D. Dalton, J. Groll, Additive manufacturing of scaffolds with sub-micron filaments via melt electrospinning writing, Biofabrication 7(3) (2015) 035002.

[40] D.G. Castner, B.D. Ratner, Biomedical surface science: Foundations to frontiers, Surface Science 500(1-3) (2002) 28-60.

[41] Y.P. Jiao, F.Z. Cui, Surface modification of polyester biomaterials for tissue engineering, Biomedical Materials 2 (2007) R24.

[42] N.D. Luong, I.S. Moon, D.S. Lee, Y.K. Lee, J.D. Nam, Surface modification of poly (I-lactide) electrospun fibers with nanocrystal hydroxyapatite for engineered scaffold applications, Materials Science and Engineering: C 28(8) (2008) 1242-1249.

[43] G. Sui, X. Yang, F. Mei, X. Hu, G. Chen, X. Deng, S. Ryu, Poly-L-lactic acid/hydroxyapatite hybrid membrane for bone tissue regeneration, Journal of Biomedical Materials Research Part A 82(2) (2007) 445-454.

[44] X.L. Deng, G. Sui, M.L. Zhao, G.Q. Chen, X.P. Yang, Poly (L-lactic acid)/hydroxyapatite hybrid nanofibrous scaffolds prepared by electrospinning, Journal of Biomaterials Science, Polymer Edition 18(1) (2007) 117-130.

[45] I. Jun, S. Jeong, H. Shin, The stimulation of myoblast differentiation by electrically conductive submicron fibers, Biomaterials 30(11) (2009) 2038-2047.

[46] X. Lv, Z. Li, S. Chen, M. Xie, J. Huang, X. Peng, R. Yang, H. Wang, Y. Xu, C. Feng, Structural and functional evaluation of oxygenating keratin/silk fibroin scaffold and initial assessment of their potential for urethral tissue engineering, Biomaterials 84 (2016) 99-110.

[47] Y. Zhang, J. Venugopal, Z.M. Huang, C. Lim, S. Ramakrishna, Characterization of the surface biocompatibility of the electrospun PCL-collagen nanofibers using fibroblasts, Biomacromolecules 6(5) (2005) 2583-2589.

[48] Z.D. Qi, T. Saito, Y. Fan, A. Isogai, Multifunctional Coating Films by Layer-by-Layer Deposition of Cellulose and Chitin Nanofibrils, Biomacromolecules (2012) 553-558.

[49] M. Dadsetan, T. Guda, M.B. Runge, D. Mijares, R.Z. LeGeros, J.P. LeGeros, D.T. Silliman, L. Lu, J.C. Wenke, P.R.B. Baer, Effect of calcium phosphate coating and rhBMP-2 on bone regeneration in rabbit calvaria using poly (propylene fumarate) scaffolds, Acta biomaterialia 18 (2015) 9-20. 
[50] K. Zhang, H. Zheng, S. Liang, C. Gao, Aligned PLLA nanofibrous scaffolds coated with graphene oxide for promoting neural cell growth, Acta biomaterialia 37 (2016) 131-142.

[51] D. Lin, K. Yang, W. Tang, Y. Liu, Y. Yuan, C. Liu, A poly (glycerol sebacate)-coated mesoporous bioactive glass scaffold with adjustable mechanical strength, degradation rate, controlled-release and cell behavior for bone tissue engineering, Colloids and Surfaces B: Biointerfaces 131 (2015) 1-11.

[52] L. Nie, D. Chen, J. Fu, S. Yang, R. Hou, J. Suo, Macroporous biphasic calcium phosphate scaffolds reinforced by poly-L-lactic acid/hydroxyapatite nanocomposite coatings for bone regeneration, Biochemical Engineering Journal 98 (2015) 29-37.

[53] F.S. Denes, S. Manolache, Macromolecular plasma-chemistry: an emerging field of polymer science, Progress in polymer science 29(8) (2004) 815-885.

[54] R. Morent, N. De Geyter, T. Desmet, P. Dubruel, C. Leys, Plasma Surface Modification of Biodegradable Polymers: A Review, Plasma Processes and Polymers (2011).

[55] X. Zhu, K.S. Chian, M.B.E. Chan-Park, S.T. Lee, Effect of argon-plasma treatment on proliferation of human-skin-derived fibroblast on chitosan membrane in vitro, Journal of Biomedical Materials Research Part A 73(3) (2005) 264-274.

[56] K. Park, Y.M. Ju, J.S. Son, K.D. Ahn, D.K. Han, Surface modification of biodegradable electrospun nanofiber scaffolds and their interaction with fibroblasts, Journal of Biomaterials Science, Polymer Edition 18(4) (2007) 369-382.

[57] J.J.A. Barry, D. Howard, K.M. Shakesheff, S.M. Howdle, M.R. Alexander, Using a Core-Sheath Distribution of Surface Chemistry through 3D Tissue Engineering Scaffolds to Control Cell Ingress, Advanced Materials 18(11) (2006) 1406-1410.

[58] S. Guimond, U. Schütz, B. Hanselmann, E. Körner, D. Hegemann, Influence of gas phase and surface reactions on plasma polymerization, Surface and Coatings Technology (2011).

[59] D. Correia, C. Ribeiro, G. Botelho, J. Borges, C. Lopes, F. Vaz, S. Carabineiro, A. Machado, S. Lanceros-Méndez, Superhydrophilic poly (I-lactic acid) electrospun membranes for biomedical applications obtained by argon and oxygen plasma treatment, Applied Surface Science 371 (2016) 74-82.

[60] L. Barbarash, E.N. Bolbasov, L. Antonova, V. Matveeva, E. Velikanova, E.V. Shesterikov, Y. Anissimov, S.I. Tverdokhlebov, Surface modification of poly- $\varepsilon$-caprolactone electrospun fibrous scaffolds using plasma discharge with sputter deposition of a titanium target, Materials Letters 171 (2016) 87-90.

[61] W. Zhu, N.J. Castro, X. Cheng, M. Keidar, L.G. Zhang, Cold atmospheric plasma modified electrospun scaffolds with embedded microspheres for improved cartilage regeneration, PloS one 10(7) (2015) e0134729.

[62] D.C. Miller, A. Thapa, K.M. Haberstroh, T.J. Webster, Endothelial and vascular smooth muscle cell function on poly (lactic-co-glycolic acid) with nano-structured surface features, Biomaterials 25(1) (2004) 53-61.

[63] F. Chen, C. Lee, S. Teoh, Nanofibrous modification on ultra-thin poly (e-caprolactone) membrane via electrospinning, Materials Science and Engineering: C 27(2) (2007) 325-332.

[64] Y. Zhu, C. Gao, X. Liu, J. Shen, Surface modification of polycaprolactone membrane via aminolysis and biomacromolecule immobilization for promoting cytocompatibility of human endothelial cells, Biomacromolecules 3(6) (2002) 1312-1319. 
[65] M. Gabriel, K. Niederer, M. Becker, C.M. Raynaud, C.-F. Vahl, H. Frey, Tailoring Novel PTFE Surface Properties: Promoting Cell Adhesion and Antifouling Properties via a Wet Chemical Approach, Bioconjugate chemistry 27(5) (2016) 1216-1221.

[66] T.C. Rothuizen, F.F. Damanik, T. Lavrijsen, M.J. Visser, J.F. Hamming, R.A. Lalai, J.M. Duijs, A.J. van Zonneveld, I.E. Hoefer, C.A. van Blitterswijk, Development and evaluation of in vivo tissue engineered blood vessels in a porcine model, Biomaterials 75 (2016) 82-90.

[67] T. Haddad, S. Noel, B. Liberelle, R. El Ayoubi, A. Ajji, G. De Crescenzo, Fabrication and surface modification of poly lactic acid (PLA) scaffolds with epidermal growth factor for neural tissue engineering, Biomatter (2016) e1231276.

[68] J.T.S. Pettikiriarachchi, C.L. Parish, D.R. Nisbet, J.S. Forsythe, Architectural and Surface Modification of Nanofibrous Scaffolds for Tissue Engineering, Nanotechnologies for the Life Sciences.

[69] H.S. Yoo, T.G. Kim, T.G. Park, Surface-functionalized electrospun nanofibers for tissue engineering and drug delivery, Advanced drug delivery reviews 61(12) (2009) 1033-1042.

[70] K.N. Chua, W.S. Lim, P. Zhang, H. Lu, J. Wen, S. Ramakrishna, K.W. Leong, H.Q. Mao, Stable immobilization of rat hepatocyte spheroids on galactosylated nanofiber scaffold, Biomaterials 26(15) (2005) 2537-2547.

[71] Z. Ma, M. Kotaki, T. Yong, W. He, S. Ramakrishna, Surface engineering of electrospun polyethylene terephthalate (PET) nanofibers towards development of a new material for blood vessel engineering, Biomaterials 26(15) (2005) 2527-2536.

[72] W. Cui, X. Li, J. Chen, S. Zhou, J. Weng, In situ growth kinetics of hydroxyapatite on electrospun poly (DL-lactide) fibers with gelatin grafted, Crystal Growth and Design 8(12) (2008) 4576-4582.

[73] A. Bhattacharya, B. Misra, Grafting: a versatile means to modify polymers: techniques, factors and applications, Progress in polymer science 29(8) (2004) 767-814.

[74] Z. Xu, L. Wan, X. Huang, Surface Modification by Graft Polymerization, Surface Engineering of Polymer Membranes (2009) 80-149.

[75] P.X. Ma, R. Zhang, Synthetic nano-scale fibrous extracellular matrix, (1999).

[76] H. Park, C. Cannizzaro, G. Vunjak-Novakovic, R. Langer, C.A. Vacanti, O.C. Farokhzad, Nanofabrication and microfabrication of functional materials for tissue engineering, Tissue engineering 13(8) (2007) 1867-1877.

[77] S. Agarwal, A. Greiner, J.H. Wendorff, Electrospinning of manmade and biopolymer nanofibersProgress in techniques, materials, and applications, Advanced functional materials 19(18) (2009) 28632879.

[78] J. Venugopal, S. Ramakrishna, Applications of polymer nanofibers in biomedicine and biotechnology, Applied biochemistry and biotechnology 125(3) (2005) 147-157.

[79] F. Yang, R. Murugan, S. Wang, S. Ramakrishna, Electrospinning of nano/micro scale poly (L-lactic acid) aligned fibers and their potential in neural tissue engineering, Biomaterials 26(15) (2005) 2603-2610.

[80] G.A. Silva, C. Czeisler, K.L. Niece, E. Beniash, D.A. Harrington, J.A. Kessler, S.I. Stupp, Selective differentiation of neural progenitor cells by high-epitope density nanofibers, Science 303(5662) (2004) 1352-1355.

[81] C.E. Semino, J. Kasahara, Y. Hayashi, S. Zhang, Entrapment of migrating hippocampal neural cells in three-dimensional peptide nanofiber scaffold, Tissue engineering 10(3-4) (2004) 643-655. 
[82] F. Gelain, D. Bottai, A. Vescovi, S. Zhang, Designer self-assembling peptide nanofiber scaffolds for adult mouse neural stem cell 3-dimensional cultures, PLoS One 1(1) (2006) e119.

[83] S. Sur, E.T. Pashuck, M.O. Guler, M. Ito, S.I. Stupp, T. Launey, A hybrid nanofiber matrix to control the survival and maturation of brain neurons, Biomaterials (2011).

[84] A. Raspa, A. Marchini, R. Pugliese, M. Mauri, M. Maleki, R. Vasita, F. Gelain, A biocompatibility study of new nanofibrous scaffolds for nervous system regeneration, Nanoscale 8(1) (2016) 253-265.

[85] S. Das, M. Sharma, D. Saharia, K.K. Sarma, M.G. Sarma, B.B. Borthakur, U. Bora, In vivo studies of silk based gold nano-composite conduits for functional peripheral nerve regeneration, Biomaterials 62 (2015) 66-75.

[86] C. Chung, J.A. Burdick, Engineering cartilage tissue, Advanced drug delivery reviews 60(2) (2008) 243-262.

[87] Y. Wang, U.J. Kim, D.J. Blasioli, H.J. Kim, D.L. Kaplan, In vitro cartilage tissue engineering with 3D porous aqueous-derived silk scaffolds and mesenchymal stem cells, Biomaterials 26(34) (2005) 7082-7094. [88] W.J. Li, R. Tuli, C. Okafor, A. Derfoul, K.G. Danielson, D.J. Hall, R.S. Tuan, A three-dimensional nanofibrous scaffold for cartilage tissue engineering using human mesenchymal stem cells, Biomaterials 26(6) (2005) 599-609.

[89] J. Kisiday, M. Jin, B. Kurz, H. Hung, C. Semino, S. Zhang, A. Grodzinsky, Self-assembling peptide hydrogel fosters chondrocyte extracellular matrix production and cell division: implications for cartilage tissue repair, Proceedings of the National Academy of Sciences 99(15) (2002) 9996.

[90] X. Mo, H.J. Weber, Electrospinning P (LLA-CL) Nanofiber: A Tubular Scaffold Fabrication with Circumferential Alignment, Wiley Online Library, 2004, pp. 413-416.

[91] B.W. Tillman, S.K. Yazdani, S.J. Lee, R.L. Geary, A. Atala, J.J. Yoo, The in vivo stability of electrospun polycaprolactone-collagen scaffolds in vascular reconstruction, Biomaterials 30(4) (2009) 583-588.

[92] R. Rhoades, R.G. Pflanzer, Human physiology, Saunders College Pub1996.

[93] D. Nisbet, J. Forsythe, W. Shen, D. Finkelstein, M. Horne, Review paper: a review of the cellular response on electrospun nanofibers for tissue engineering, Journal of biomaterials applications 24(1) (2009) 7-29.

[94] S. Kimakhe, S. Bohic, C. Larrose, A. Reynaud, P. Pilet, B. Giumelli, D. Heymann, G. Daculsi, Biological activities of sustained polymyxin $B$ release from calcium phosphate biomaterial prepared by dynamic compaction: An in vitro study, Journal of biomedical materials research 47(1) (1999) 18-27.

[95] H. Yoshimoto, Y. Shin, H. Terai, J. Vacanti, A biodegradable nanofiber scaffold by electrospinning and its potential for bone tissue engineering, Biomaterials 24(12) (2003) 2077-2082.

[96] M. Shin, H. Yoshimoto, J.P. Vacanti, In vivo bone tissue engineering using mesenchymal stem cells on a novel electrospun nanofibrous scaffold, Tissue engineering 10(1-2) (2004) 33-41.

[97] C. Li, C. Vepari, H.J. Jin, H.J. Kim, D.L. Kaplan, Electrospun silk-BMP-2 scaffolds for bone tissue engineering, Biomaterials 27(16) (2006) 3115-3124.

[98] J. Kim, M.H.R. Magno, H. Waters, B.A. Doll, S. McBride, P. Alvarez, A. Darr, A. Vasanji, J. Kohn, J.O. Hollinger, Bone Regeneration in a Rabbit Critical-Size Calvarial Model Using Tyrosine-Derived Polycarbonate Scaffolds, Tissue engineering (ja) (2012). 
[99] J. Xie, C. Peng, Q. Zhao, X. Wang, H. Yuan, L. Yang, K. Li, X. Lou, Y. Zhang, Osteogenic differentiation and bone regeneration of iPSC-MSCs supported by a biomimetic nanofibrous scaffold, Acta biomaterialia 29 (2016) 365-379.

[100] C. Dhand, S.T. Ong, N. Dwivedi, S.M. Diaz, J.R. Venugopal, B. Navaneethan, M.H. Fazil, S. Liu, V. Seitz, E. Wintermantel, Bio-inspired in situ crosslinking and mineralization of electrospun collagen scaffolds for bone tissue engineering, Biomaterials 104 (2016) 323-338.

[101] L. Moroni, R. Licht, J. De Boer, J.R. De Wijn, C.A. Van Blitterswijk, Fiber diameter and texture of electrospun PEOT/PBT scaffolds influence human mesenchymal stem cell proliferation and morphology, and the release of incorporated compounds, Biomaterials 27(28) (2006) 4911-4922.

[102] C.L. Casper, J.S. Stephens, N.G. Tassi, D.B. Chase, J.F. Rabolt, Controlling surface morphology of electrospun polystyrene fibers: effect of humidity and molecular weight in the electrospinning process, Macromolecules 37(2) (2004) 573-578.

[103] J.E. Phillips, T.A. Petrie, F.P. Creighton, A.J. García, Human mesenchymal stem cell differentiation on self-assembled monolayers presenting different surface chemistries, Acta biomaterialia 6(1) (2010) 1220. 


\title{
Chapter 2
}

\section{Tailoring Chemical and Physical Properties of Fibrous Scaffolds from Block Copolyesters Containing Ether and Thio- ether Linkages for Skeletal Differentiation of Human Mesenchymal Stromal Cells}

\begin{abstract}
Honglin Chen ${ }^{a}$, Matteo Giglib, Chiara Gualandic, Roman Truckenmüllera, Clemens van Blitterswijk ${ }^{a}$,
\end{abstract} Nadia Lotti ${ }^{b}$, Andrea Munari ${ }^{b}$, Maria Letizia Focarete ${ }^{c, d}$, Lorenzo Moroni ${ }^{a}$

aDepartment of Complex Tissue Regeneration

MERLN Institute for Technology Inspired Regenerative Medicine

6200 MD Maastricht, The Netherlands

${ }^{b}$ Department of Civil, Chemical, Environmental and Materials Engineering

University of Bologna, 40127 Bologna, Italy

"Department of Chemistry "G. Ciamician" and INSTM UdR of Bologna

University of Bologna,40126 Bologna, Italy

dHealth Sciences and Technologies - Interdepartmental Center for Industrial Research (HST-ICIR), University of Bologna, 40064 - Ozzano dell'Emilia (Bologna), Italy 


\section{Abstract}

Bioactive scaffolds for tissue engineering call for demands on new materials which can enhance traditional biocompatibility requirements previously considered for clinical implantation. The current commercially available thermoplastic materials, such as poly(lactic acid) (PLA), poly(glycolic acid) (PGA), poly( $\varepsilon$-caprolactone) (PCL) and their copolymers, have been used to fabricate scaffolds for regenerative medicine. However, these polymers have limitations including lacking of broadly tuning mechanical and degradable properties, and activation of specific cell-scaffold interactions, which limit their further application in tissue engineering. In the present study, electrospun scaffolds were successfully fabricated from a new class of block poly(butylene succinate)-based (PBSbased) copolyesters containing either butylene thiodiglycolate (BTDG) or butylene diglycolate (BDG) sequences. The polyesters displayed tunable mechanical properties and hydrolysis rate depending on the molecular architecture and on the kind of heteroatom introduced along the polymer backbone. To investigate their potential for skeletal regeneration, human mesenchymal stromal cells (hMSCs) were cultured on the scaffolds in basic, osteogenic and chondrogenic media. Our results demonstrated that PBS-based copolyesters containing thio-ether linkages (i.e. BTDG segments) were more favorable for chondrogenesis of hMSCs than those containing ether linkages (i.e. BDG sequences). In contrast, PBS-based copolyesters containing ether linkages showed enhanced mineralization. Therefore, these new functional scaffolds might hold, potential for osteochondral tissue engineering applications.

\section{Keywords:}

Aliphatic copolyesters, ether and thioether linkages, electrospun scaffolds, human mesenchymal stromal cells, skeletal differentiation 


\subsection{Introduction}

A promising strategy for repairing damaged tissues and organs is to design scaffolds able to support and eventually control cell activity, i.e. adhesion, proliferation and differentiation. Electrospinning is a simple technique capable of producing non-woven scaffolds made of continuous fibers with diameters ranging from few tens of nanometers up to several micrometers[1]. The resulting fibrillary networks mimic the morphology of native extracellular matrix (ECM), which serves to organize cells and provides signals for cellular responses [2].

Among the manifold of current commercially available thermoplastic materials used to fabricate three-dimensional (3D) scaffolds for regenerative medicine, aliphatic polyesters undoubtedly represent so far the most extensive studied class, since they combine good physico-chemical and mechanical properties with assessed biocompatibility[3]. Poly(lactic acid) (PLA), poly(glycolic acid) (PGA), poly( $\varepsilon$-caprolactone) (PCL) and their copolymers are typically used[4] and their hydrolytic and enzymatic degradation under physiological conditions has been extensively proven[3]. Although these polymers have found many applications in clinical practice, the wide tuning of their mechanical, physico-chemical and biological properties is somehow limited[4]. For instance, the copolymerization of PLA with PCL enables to decrease polymer elastic modulus, thus obtaining more deformable materials that, however, are highly hydrophobic and display a very slow degradation rate. Hydrophilicity and hydrolysis rate can be significantly enhanced through copolymerization of PLA with PGA but, in this case, the rigidity of the material persists. This hampers the fabrication of 3D scaffolds capable of adapting to the needs of the specific tissue to be regenerated. Therefore, new polyesters with more flexibility in tuning their properties attract considerable attention in regenerative medicine.

In the last few years, an increasing number of researchers proposed poly(butylene succinate) (PBS) as a possible alternative to the above mentioned aliphatic polyesters due to the following advantages: (i) simple synthetic strategy through melt polycondensation; (ii) low cost of raw materials (1,4-butanediol and succinic acid), which can be also obtained from renewable resources[5]; (iii) high melting temperatures among poly(alkylene dicarboxylate)s $\left(T_{m}=113-115^{\circ} \mathrm{C}\right)$; (iv) good thermal stability (thus a wide processing temperature range); (v) proven biodegradability and biocompatibility[6]. Furthermore, PBS is already commercialized by different companies for various applications such as compost bags and mulching films, but also fishery, civil engineering and construction and for common household goods. 
Many efforts have been recently devoted to target PBS modification aiming to achieve better performances with respect to the intended application. For biomedical applications, composites have been realized by incorporating organic or inorganic fillers such as chitosan, collagen, calcium phosphate or hydroxyapatite along the PBS macromolecular chain[6]. A plethora of PBS-based blends and copolymers have been also obtained[6]. Among other copolymerization techniques, reactive blending is a versatile and solvent free approach which permits to prepare block copolymers of different molecular architecture by simply varying the reaction time [7].

Recent studies have demonstrated that the properties of scaffold, such as scaffold elasticity, structure and organization, and chemistry, can direct stem cell differentiation into specific lineage[8-10]. In this study, electrospun scaffolds fabricated from a class of block PBS-based copolyesters containing either butylene thiodiglycolate (BTDG) or butylene diglycolate (BDG) sequences, obtained by reactive blending, are presented. In particular, the possibility to use the synthesized copolymers as an alternative to conventional polyesters was explored to achieve enhanced control over stem cell differentiation. In these systems, the addition of heteroatoms along the macromolecular chains has been demonstrated to tune chain flexibility, thus affecting polymer crystallinity and mechanical properties of aliphatic polyesters [11,12]. Moreover, through this strategy surface hydrophilicity has been modified, due to the presence of electronegative oxygen and sulphur atoms[13-15]. All these factors significantly impact the biodegradation rate and biocompatibility of the final materials [16, 17]. Scaffolds with tailored chemical composition and physical properties have been prepared and characterized. Their hydrolytic degradation profile has been evaluated under physiological conditions. Finally, we show that these copolymers may have the potential to steer stem cell differentiation towards skeletal lineages.

\subsection{Materials and Methods}

\subsubsection{Materials}

Dimethylsuccinate (DMS), 1,4-butanediol (BD), diglycolic acid (DGA), thiodiglycolic acid (TDGA), and titanium tetrabutoxide (TBT) (Sigma-Aldrich) were reagent grade products. All the reagents were used as supplied with the exception of TBT which was distilled before use. Chloroform, dichloromethane (DCM), 2-chloroethanol (CE) and hexafluoro-2propanol (HFIP) were purchased from Sigma-Aldrich and were used without any further purification. 


\subsubsection{Polymer synthesis}

Starting homopolymers, i.e. PBS, poly(butylene diglycolate) (PBDG) and poly(butylene thiodiglycolate) (PBTDG) were synthesized in bulk by the usual two steps melt polycondensation, starting from 1,4-butanediol (BD) and DMS, DGA or TDGA, as reported elsewhere[11, 12]. Two blends, PBS/PBDG and PBS/PBTDG, were obtained by melt mixing for 10 min equimolar amounts of PBS with PBDG and PBTDG, respectively, according to the procedures previously described [11,12]. Poly(butylene succinate/diglycolate) $\left(\mathrm{P}\left(\mathrm{BS}_{11} \mathrm{BDG}_{11}\right)\right)$ and poly(butylene succinate/thiodiglycolate) $\left(\mathrm{P}\left(\mathrm{BS}_{10} \mathrm{BTDG}_{10}\right)\right)$ copolyesters were obtained by melt mixing equimolar amounts of $\mathrm{PBS}$ with either PBDG (for $30 \mathrm{~min}$ ) or PBTDG (for $45 \mathrm{~min}$ ), respectively[11, 12]. The reactions were carried out at $225^{\circ} \mathrm{C}$, under dry nitrogen atmosphere to prevent polymer degradation. Copolymer formation was catalysed by the residual titanium tetrabutoxide used for the synthesis of the parent homopolymers. The reactive blending provided two block copolymers $\left(P\left(B S_{11} B D G_{11}\right)\right.$ and $\left.P\left(B S_{10} B T D G_{10}\right)\right)$ with similar block length.

\subsubsection{Scaffold and film preparation}

PBS, PBS/PBDG and PBS/PBTDG blends and $P\left(B S_{11} B D G_{11}\right)$ and $P\left(B S_{10} B D_{D G}\right)$ copolymers were subjected to electrospinning to obtain 3D mats. Scaffolds were produced by using a customized electrospinning apparatus, comprised of a high-voltage power supply (SL 50 P 10/CE/230; Spellman), a syringe pump (KD Scientific 200 series), a glass syringe, a stainless steel blunt-ended needle (inner diameter $=0.84 \mathrm{~mm}$ ) connected with the power supply electrode, and a grounded aluminium plate-type collector $(7 \mathrm{~cm} \times 7 \mathrm{~cm})$. Polymer solution was dispensed through a Teflon tube to the needle which was placed vertically on the collecting plate at a measured distance (D). The scaffolds were produced at room temperature (RT) and a relative humidity of $40 \pm$ $5 \%$. The electrospun solution and the operating conditions for each polymer were selected after optimization experiments aimed at obtaining bead-free fibers and are reported in Table S1. Electrospun mats were kept under vacuum over $\mathrm{P}_{2} \mathrm{O}_{5}$ at $\mathrm{RT}$ overnight to remove residual solvents.

Films ( $0.2 \mathrm{~mm}$ thick) of PBS, blends and copolymers were obtained by compression moulding the polymers between Teflon plates, with an appropriate spacer, at $140^{\circ} \mathrm{C}$ for 1 min under a pressure of 2 ton $\mathrm{m}^{-2}$ (Carver $\mathrm{C} 12$, laboratory press). 


\subsubsection{Material characterization}

Polymer structure and composition were determined by means of proton nuclear magnetic resonance $\left({ }^{1} \mathrm{H}-\mathrm{NMR}\right)$ spectroscopy, whereas the distribution of the comonomeric sequences along the polymer chain was evaluated by ${ }^{13} \mathrm{C}-\mathrm{NMR}$ spectroscopy. The measurements were carried out at RT by employing a Varian Inova $400-\mathrm{MHz}$ instrument (Agilent Technologies, USA). Molecular weights were evaluated by gel permeation chromatography (GPC) at $30^{\circ} \mathrm{C}$ using a $1100 \mathrm{HPLC}$ system (Agilent Technologies, USA) equipped with PLgel 5- $\mu$ m MiniMIX-C column (Agilent Technologies, USA). A refractive index was employed as detector. Chloroform was used as eluent with a $0.3 \mathrm{~mL} / \mathrm{min}$ flow and sample concentrations of about $2 \mathrm{mg} / \mathrm{mL}$. A molecular weight calibration curve was obtained with polystyrene standards in the range of molecular weight 2000-100,000 g/mol. Thermogravimetric analysis (TGA) was carried out under nitrogen atmosphere using a Perkin Elmer TGA7 apparatus (gas flow: $30 \mathrm{~mL} / \mathrm{min}$ ) at $10^{\circ} \mathrm{C} / \mathrm{min}$ heating rate, up to $900{ }^{\circ} \mathrm{C}$. Calorimetric measurements were conducted by using a Perkin Elmer DSC7 instrument. In the typical setup, the external block temperature control was set at $-120^{\circ} \mathrm{C}$ and weighed samples of c.a. $10 \mathrm{mg}$ were heated up to $40{ }^{\circ} \mathrm{C}$ above fusion temperature at a rate of $20{ }^{\circ} \mathrm{C} / \mathrm{min}$. Scanning electron microscopic (SEM) observations were carried out using a Philips 515 SEM (FEl, Eindhoven, The Netherlands) at an accelerating voltage of $15 \mathrm{kV}$, on samples sputter coated with gold. The distribution of fibre diameters was determined through the measurement of approximately 150 fibres by means of an acquisition and image analysis software (EDAX Genesis, New York, USA), and the results were given as the average diameter \pm standard deviation. Static contact angle measurements were performed on polymer films using a KSV CAM101 (KSV, Espoo, Finland) instrument at ambient conditions, by recording the side profiles of deionized water drops for image analysis. Five drops were observed on different areas for each film, and contact angles were reported as the average value \pm standard deviation. Stress-strain measurements were performed with an Instron 4465 tensile testing machine equipped with a $100 \mathrm{~N}$ load cell on rectangular films, according to ASTM D882-02 standard. Sample were $5 \mathrm{~mm}$ wide, the gauge length was $20 \mathrm{~mm}$ and the cross-head speed was $5 \mathrm{~mm} / \mathrm{min}$. Load-displacement curves were obtained and converted to stress-strain curves. At least six replicate specimens were run for each sample and results were provided as the average value \pm standard deviation. 


\subsubsection{Hydrolytic degradation studies}

Hydrolytic degradation studies were carried out on rectangular mats of PBS, blends and copolymers in triplicate. Prior to degradation experiments, each specimen was dried over $\mathrm{P}_{2} \mathrm{O}_{5}$ under vacuum at $\mathrm{RT}$, and weighed to obtain the sample initial mass. Mats were individually immersed in phosphate buffered solution $(0.1 \mathrm{M}, \mathrm{pH}=7.4)$ and incubated in a SW22 Julabo shaking water bath at $37{ }^{\circ} \mathrm{C}$ and $50 \mathrm{rpm}$. The buffer solution was periodically changed to keep the $\mathrm{pH}$ constant during the entire time scale of the degradation experiments. At different time intervals, triplicate specimens for each sample were recovered from the bath, repeatedly washed with deionized water and dried over $\mathrm{P}_{2} \mathrm{O}_{5}$ under vacuum to constant weight. The mass loss was determined gravimetrically by comparing the dry weight remaining at a specific time with the initial sample weight. The molecular weight of each recovered sample was determined by GPC. ${ }^{1} \mathrm{H}-\mathrm{NMR}$ analysis was performed to detect changes in the molecular composition during the hydrolysis experiments and differential scanning calorimetry (DSC) was used to evaluate the change in crystallinity degree.

\subsubsection{Cell culture and seeding}

Human mesenchymal stromal cells (hMSCs, donor No. 8001L) were provided from Texas A\&M Health Science Center, which has supplied standardized preparations of MSCs to hundreds of laboratories under the auspices of an NIH/NCRR grant (P40 RR 17447-06). Briefly, human bone marrow aspirates were obtained under a protocol approved by an institutional review board. Mononuclear cells were separated from bone marrow aspirate using density centrifugation. They were plated to achieve adherent hMSCs and harvested when cells reached $60-80 \%$ confluence. These cells were called passage zero (P0) cells and were further expanded, harvested and frozen at passage one (P1). Prior to release, the frozen P1 cells were analyzed over three passages to assess successful expression of MSCs surface markers[18, 19]. To achieve sufficient cells for seeding onto scaffolds, $\mathrm{P} 1$ cells were expanded in proliferation medium (PM), consisting of a-MEM (Gibco), 10\% fetal bovine serum (Lonza), $2 \mathrm{mM}$ L-glutamin (Gibco), $0.2 \mathrm{mM}$ ascorbic acid (SigmaAldrich), $100 \mathrm{U} / \mathrm{mL}$ penicillin and $100 \mathrm{mg} / \mathrm{mL}$ streptomycin (Gibco) and $1 \mathrm{ng} / \mathrm{mL}$ recombinant basic fibroblast growth factor (bFGF, Instruchemie). Cells were harvested for further sub-cultures when they became $80-90 \%$ confluence. Electrospun scaffolds were placed in cell culture plates and pressed with rubber O-rings (Eriks BV, The Netherlands) to prevent them from floating. The specimens were sterilized by immersion in $70 \%(\mathrm{v} / \mathrm{v})$ 
ethanol for $15 \mathrm{~min}$ for three times. Subsequently, scaffolds were washed for $1 \mathrm{~min}$ with PBS for three times and immersed in basic medium (BM) overnight before cell seeding. A density of $5 \times 10^{3}$ cells $/ \mathrm{cm}^{2}$ was used to seed hMSCs onto electrospun scaffolds. Cellscaffold constructs were cultured for up to 28 days in BM consisting of PM without bFGF, osteogenic medium (OM) comprising BM supplemented with $1 \times 10^{-6} \mathrm{M}$ dexamethasone), mineralization medium ( $\mathrm{MM}$ ) composed of $\mathrm{OM}$ plus b-glycerol phosphate, and chondrogenic medium (CM) containing DMEM (Gibco,) supplemented with $1 \%$ ITS premix (Micronic BV), $50 \mu \mathrm{g} / \mathrm{mL}$ ascorbic acid (Sigma-Aldrich), 100 units/mL penicillin (Life technologies), $100 \mu \mathrm{g} / \mathrm{mL}$ streptomycin (Life technologies), $100 \mathrm{nM}$ dexamethasone (Sigma-Aldrich), $40 \mu \mathrm{g} / \mathrm{mL}$ proline (Sigma-Aldrich), $100 \mu \mathrm{g} / \mathrm{mL}$ sodium pyruvate (Life technologies), $0.01 \mu \mathrm{g} / \mathrm{mL}$ TGF $\beta 1$ (R\&D systems). Culture experiments were performed in an incubator with $5 \% \mathrm{CO}_{2}$ humid atmosphere at $37^{\circ} \mathrm{C}$. Culture medium was refreshed every 2 days.

\subsubsection{Metabolic activity}

Cell culture medium was removed from cell-scaffold constructs and supplemented with a medium containing $10 \%(\mathrm{v} / \mathrm{v})$ PrestoBlue ${ }^{\mathrm{TM}}$ reagent (Life technologies). The cell-scaffold constructs were incubated at $37{ }^{\circ} \mathrm{C}$ for $1 \mathrm{~h}$ in the dark. Fluorescence was measured at $590 \mathrm{~nm}$ using a multi-label plate reader (Perkin Elmer Victor3 1420, USA).

\subsubsection{Cell morphology analysis}

Cell morphology and attachment on scaffolds was determined by SEM using an XL 30 ESEM-FEG (Philips, The Netherlands). Briefly, samples were rinsed twice with PBS, fixed with $10 \%$ formalin for $2 \mathrm{~h}$. After washing with PBS two times, the samples were dehydrated with a gradient ethanol series, dried by using critical point drying (Balzers CPD 030) and sputtered with gold (Cressington) before SEM analysis.

\subsubsection{Quantification of $D N A$}

Samples were washed with PBS three times and stored at $-80^{\circ} \mathrm{C}$ overnight. After thawing, samples were lysated for $16 \mathrm{~h}$ with a Tris/EDTA buffer containing $1 \mathrm{mg} / \mathrm{mL}$ proteinase $\mathrm{K}$, $185 \mu \mathrm{g} / \mathrm{mL}$ iodoacetamine (Sigma-Aldrich) and $10 \mu \mathrm{g} / \mathrm{mL}$ Pepstatin A (Sigma-Aldrich). Subsequently, the quantification of the total amount of DNA was examined by using the CyQuant ${ }^{\circledR}$ DNA assay (Molecular Probes, Eugene, USA) as previously reported[20]. Lysate was pipetted in duplicate to a black 96-well plate, followed by addition of $\mathrm{NaCl}$ EDTA buffer containing component B of the kit (20x) and RNase (1000x). The plate was 
incubated for $1 \mathrm{~h}$ at RT. Subsequently, Gr-dye solution was added and the samples were incubated for $15 \mathrm{~min}$. The fluorescence signal was determined using a spectrophotometer (Victor3 1420, Perkin Elmer, USA) at an excitation wavelength of $480 \mathrm{~nm}$ and emission wavelength of $520 \mathrm{~nm}$.

\subsubsection{Alkaline phosphatase activity (ALP) assay}

The protocol of measuring ALP activity was similar to a previous study[21]. Briefly, the cell-scaffold constructs were digested for $1 \mathrm{~h}$ at $\mathrm{RT}$ in a cell lysis buffer at a $\mathrm{pH} 7.8$ composed of $0.1 \mathrm{M} \mathrm{KH}_{2} \mathrm{PO}_{4}, 0.1 \mathrm{M} \mathrm{K}_{2} \mathrm{HPO}_{4}$ and $0.1 \%$ Triton $\mathrm{X}-100$. Then, cell lysates were pipetted in duplicate to 96-well plate. After addition of CDP-star ${ }^{\circledR}$ (Roche Life Science), the lysate were incubated for $15 \mathrm{~min}$ at RT. The measurements were performed on a Victor3 1420 (Perkin Elmer, USA) plate reader. The value of ALP activity was normalized to DNA quantity per scaffold.

\subsubsection{Glycosaminoglycan (GAG) assay}

Cell-scaffold constructs were washed with PBS three times and stored at $-80{ }^{\circ} \mathrm{C}$ for at least $24 \mathrm{~h}$. After thawing, the constructs were digested for $16 \mathrm{~h}$ with Tris/EDTA buffer as mentioned above. The amount of GAG was determined spectrophotometrically after reaction with $16 \mathrm{mg}$ of dimethylmethylene blue dye (DMMB, Sigma-Aldrich) in a $10 \mathrm{mM}$ hydrochloric acid solution containing $3.04 \mathrm{~g} / \mathrm{L}$ of glycine and $2.37 \mathrm{~g} / \mathrm{L}$ of $\mathrm{NaCl}$. The absorbance was measured on a micro plate reader (Multiskan GO, Thermo Fisher, USA). The amount of GAG was calculated using a standard of chondroitin sulfate (SigmaAldrich).

\subsubsection{Histological staining}

ALP staining was carried out by using an alkaline phosphatase kit (Sigma-Aldrich). Samples were fixed in $40 \%$ citrate working solution in acetone for $30 \mathrm{~s}$ and rinsed with deionized water three times. Alkaline dye mixture was added and incubated for $30 \mathrm{~min}$ at $\mathrm{RT}$ in the dark. Samples were finally rinsed thoroughly with deionized water three times. For alizarin red staining (ARS) assay, after fixation in $10 \%$ formalin for $1 \mathrm{~h}$, samples were rinsed with PBS three times, washed twice with distilled water and stained with a freshly filtered $2 \%$ aqueous Alizarin red solution $(\mathrm{pH} \mathrm{4.2)}$ for $2 \mathrm{~min}$. The excess stain was washed off thoroughly with distilled water. To quantify the orange-red coloration of ARS, the protocol was performed as described in a previous study [22, 23]. After removing excess water, samples were transferred to a microcentrifuge tube. A $10 \%$ acetic acid 
solution was added and incubated for $30 \mathrm{~min}$ at RT with shaking. The slurry was centrifuged for $15 \mathrm{~min}$ at $20,000 \mathrm{~g}$ after vortexing for $30 \mathrm{~s}$. Then, the supernatant was transferred to a new 1.5-mL microcentrifuge tube. A $10 \%$ ammonium hydroxide was utilized to neutralize the acid. Aliquots of the supernatant were read in triplicate at $405 \mathrm{~nm}$ in 96-well format using opaque-walled, transparent-bottomed plates (Fisher Lifesciences). All the images for ALP staining and ARS staining were taken using a stereomicroscope (Nikon SMZ800 with Q-imaging Retiga 1300 camera).

\subsubsection{Quantitative PCR analysis}

To analyze the gene expression of hMSCs on scaffolds, total RNA was isolated using a combination of TRIzol ${ }^{\circledR}$ with a NucleoSpinRNAII ISOLATON ${ }^{\circledR}$ kit (Bioke). Briefly, scaffolds at day 7 and day 28 were rinsed with PBS three times and $1 \mathrm{~mL}$ of TRIzol was added to each sample. After addition of chloroform, phase separation occurred by centrifugation at $12,000 \mathrm{~g}$. The aqueous phase containing RNA was collected, mixed with equal volume of $75 \%(\mathrm{v} / \mathrm{v})$ ethanol and loaded onto an RNA binding column of the kit. After a series of purification steps, RNA was re-suspended in RNase-free water and collected using a clean Eppendorf ${ }^{\circledR}$ tube by centrifugation at $12,000 \mathrm{~g}$. The concentration and purity of RNA were measured by using a spectrophotometer (ND 1000, Nanodrop Technologies, USA). iScript (Bio-Rad, Veenendaal, the Netherlands) was used for the cDNA synthesis following the manufacturer's instruction. Quantitative polymerase chain reaction (qPCR) was carried out using SYBR-green supermix (Bio-Rad). Cycling parameters for running qPCR were set as follows: initial denaturation at $95^{\circ} \mathrm{C}$ for $10 \mathrm{~min}$, followed by 40 cycles of $15 \mathrm{~s}$ at $95^{\circ} \mathrm{C}$ and $1 \mathrm{~min}$ at $60^{\circ} \mathrm{C}$. The sequences of the analyzed primers are shown in Table S3. Data are expressed as fold change relative to controls, after normalizing to B2M.

\subsubsection{Statistical analysis}

All data points are expressed as mean \pm standard deviation. Statistical difference was determined by using two-way analysis of variance (ANOVA), followed by a Tukey's posthoc test. A value of $p<0.05$ was considered statistically significant.

\subsection{Results and discussion}

\subsubsection{Electrospinning and sample characterization}

PBS, PBDG and PBTDG displayed a similar chemical structure, all containing two ester groups per repeating unit along a saturated aliphatic chain (Figure 1). With respect to 
PBS, differences can be observed in the acid sub-units: PBDG contains an ether bond while PBTDG a thio-ether bond. We previously demonstrated that these minimal differences in the polymer backbone have a remarkable effect on polymer thermomechanical properties[11, 12]. In the present study, physical blends of PBS with PBDG and PBTDG and copolymers of PBS containing either BDG or BTDG units with long block length were considered for scaffold fabrication, since these polymeric systems possess physico-chemical and mechanical characteristics significantly different from each other and from the PBS homopolymer. The molecular characterization of the polymeric systems investigated in the present work is reported in Table 1.

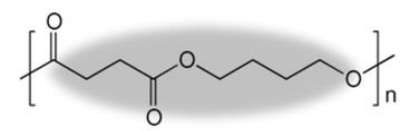

PBS

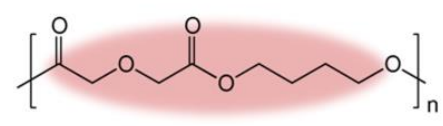

PBDG

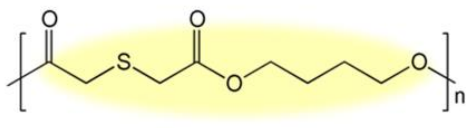

PBTDG

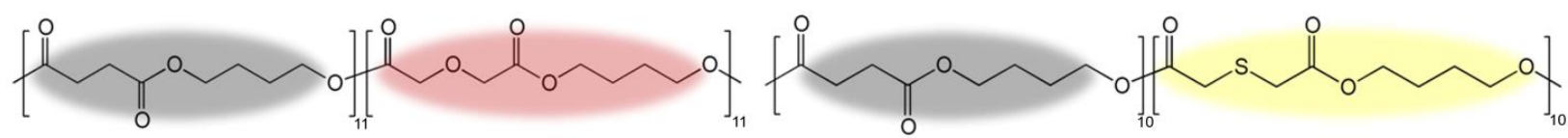

$\mathrm{P}\left(\mathrm{BS}_{11} \mathrm{BDG}_{11}\right)$

$\mathrm{P}\left(\mathrm{BS}_{10} \mathrm{BTDG}_{10}\right)$

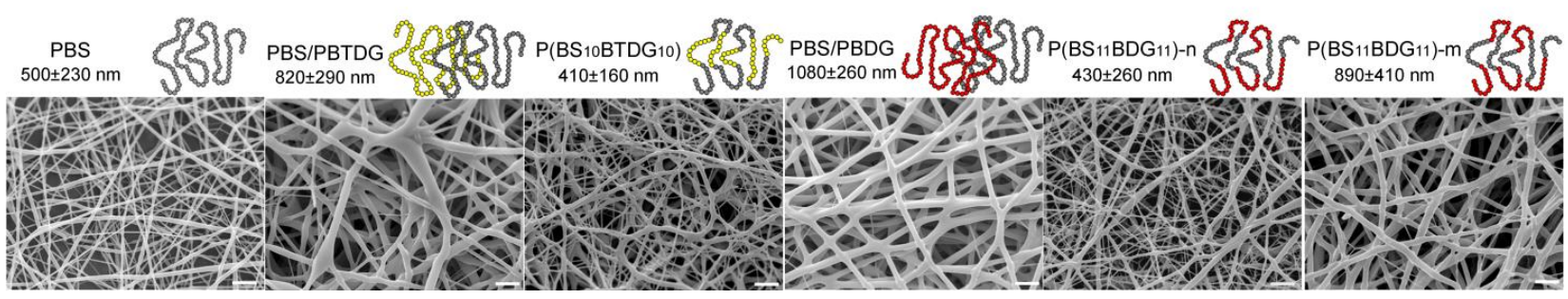

Figure 1. Polymer chemical formulas, SEM micrographs and fibre dimensions of the obtained scaffolds.

Both the blends and the copolyesters are characterized by relatively high molecular weights. This result indicates that no relevant thermal degradation occurred during the reactive blending. Looking into more detail, a slight decrease of molecular weight can be observed for the block copolymers. This result has been previously described and can be explained on the basis of two opposite effects: chain scission and transesterification reactions $[11,12]$. For short mixing times, i.e. those here employed, the chain scission reactions prevailed over transesterifications and therefore a decrease of molecular weight could be detected.

The chemical structure and composition of all copolyesters, determined by ${ }^{1} \mathrm{H}-\mathrm{NMR}$ were found to be consistent with the expected ones. ${ }^{13} \mathrm{C}-\mathrm{NMR}$ measurements were performed to study the structural changes due to the reactive blending. As far as the two 
blends are concerned, the NMR spectrum is the simple addition of PBS and PBTDG ones, indicating that no appreciable chemical reactions took place during the mixing. On the contrary, in $\mathrm{P}\left(\mathrm{BS}_{10} \mathrm{BTDG_{10 }}\right)$ and $\mathrm{P}\left(\mathrm{BS}_{11} \mathrm{BDG}_{11}\right)$ two new peaks developed in the region between 64.1 and $65.5 \mathrm{ppm}$ (where the signals due to the carbon atoms of the 1,4butanediol glycol subunit are located), due to the BS-BTDG and BS-BDG mixed sequences, respectively[11, 12]. Lastly, the average block length has been calculated from the equations previously reported [11, 12].

Electrospinning conditions parameters were optimized in order to obtain defect-free fibres. The scaffolds were highly porous with microscale interstitial pores made of randomly oriented fibres. In the case of $\mathrm{P}\left(\mathrm{BS}_{11} \mathrm{BDG}_{11}\right)$, two different polymeric solutions were prepared to obtain scaffolds with either micrometric or nanometric diameters (i.e. $890 \pm 410 \mathrm{~nm}$ and $410 \pm 160 \mathrm{~nm})$ that were labelled $P\left(B S_{11} B D G_{11}\right)-m$ and $P\left(B S_{11} B B_{11}\right)-$ $\mathrm{n}$, respectively (Figure 1 and Table S1).

Electrospun fibres were characterized by TGA and DSC. TGA did not reveal residual solvents in the fabricated fibres and confirmed that electrospinning process did not affect polymer thermal degradation behaviour. DSC was used to evaluate thermal transitions in the electrospun mats (Table 1). The calorimetric data of $P\left(B S_{11} B D G_{11}\right)-m$ and $P\left(B S_{11} B D G_{11}\right)-n$ were identical within the experimental errors. The phase behaviour of the polymers under investigation was similar. As far as the glass transition phenomenon is concerned, only one glass transition temperature $\left(T_{g}\right)$ was always clearly evident suggesting the presence of a homogeneous amorphous state. All polymers were semicrystalline, being the corresponding calorimetric traces (Figure S1) characterized by a significant melting endotherm. The overall heat of fusion $\left(\Delta \mathrm{H}_{\mathrm{m}}\right)$ decreased from blends to block copolymers because of the reduced length of crystallisable sequences. The copolymer containing ether-oxygen showed a slightly bigger $\Delta \mathrm{H}_{\mathrm{m}}$ associated to the PBS crystal phase compared to the thio-ether containing copolymer. This implied that the PBS crystallization was less hampered in the copolymer containing BDG co-units. Interestingly, in the PBS/PBDG blend two distinct melting phenomena were evident, suggesting the simultaneous presence of the crystalline phases of the two homopolymers. This result has been previously confirmed by X-ray analyses [12]. For the blends, the melting temperature $\left(\mathrm{T}_{\mathrm{m}}\right)$ was equal to that of PBS (and of PBDG homopolymer in the case of $\mathrm{PBS} / \mathrm{PBDG}$ ), while it decreased in the block copolymers because of the formation of a less perfect crystalline phase. 
Table 1. Polymer and scaffold characterization.

\begin{tabular}{|c|c|c|c|c|c|c|c|c|}
\hline Polymer & $\begin{array}{c}\mathrm{M}_{\mathrm{n}}{ }^{\mathrm{a}} \\
(\mathrm{g} / \mathrm{mol})\end{array}$ & $\mathrm{PDI}^{\mathrm{a}}$ & $\begin{array}{l}\mathrm{T}_{\mathrm{g}}^{\mathrm{b}} \\
\left({ }^{\circ} \mathrm{C}\right)\end{array}$ & $\begin{array}{c}T_{m}, B D G^{b} \\
\left({ }^{\circ} \mathrm{C}\right)\end{array}$ & $\begin{array}{c}\mathrm{T}_{\mathrm{m}, \mathrm{BS}}{ }^{\mathrm{b}} \\
\left({ }^{\circ} \mathrm{C}\right)\end{array}$ & $\begin{array}{c}\Delta \mathrm{H}_{\mathrm{m}, \mathrm{BDG}^{\mathrm{b}}} \\
(\mathrm{J} / \mathrm{g})\end{array}$ & $\begin{array}{c}\Delta \mathrm{H}_{\mathrm{m}, \mathrm{BS}}{ }^{\mathrm{b}} \\
(\mathrm{J} / \mathrm{g})\end{array}$ & $\begin{array}{c}\text { WCA }^{\mathrm{c}} \\
\left({ }^{\circ}\right)\end{array}$ \\
\hline PBS & 34600 & 2.5 & -32 & 1 & 115 & 1 & 75 & $96 \pm 1$ \\
\hline PBS/PBTDG & 37000 & 2.6 & -45 & / & 113 & I & 33 & $81 \pm 2$ \\
\hline $\mathrm{P}\left(\mathrm{BS}_{10} \mathrm{BTDG}_{10}\right)$ & 30200 & 2.5 & -44 & I & 100 & I & 30 & $79 \pm 4$ \\
\hline PBS/PBDG & 23600 & 2.8 & -29 & 63 & 114 & 28 & 34 & $72 \pm 2$ \\
\hline $\mathrm{P}\left(\mathrm{BS}_{11} \mathrm{BDG}_{11}\right)$ & 19700 & 2.3 & -30 & I & 106 & l & 35 & $74 \pm 2$ \\
\hline
\end{tabular}

a) by GPC performed on polymers in form of powder; b) by DSC performed on polymeric scaffolds; c) by WCA performed on polymers in form of film

To investigate the effect of polymer chemistry on material hydrophilicity, water contact angle (WCA) measurements were performed on hot-pressed films and the results reported in Table 1. The significant decrease of WCA values in blends and copolymers was due to the introduction of highly electronegative atoms ( $O$ and $S$ ) along the PBS polymeric chain, which has the effect of increasing material surface wettability, as previously reported for other polymeric systems[13-17, 24]. The polymers containing BDG repeating units displayed a lower WCA than those containing BTDG units. Thus, the former were more hydrophilic as a consequence of the higher electronegativity of oxygen atoms with respect to sulphur ones.

In order to correlate bulk mechanical properties with polymer chemical structure, tensile stress-strain measurements were carried out on polymeric films rather than electrospun fibers. Indeed, it is somewhat difficult to make a direct comparison among stress-strain data of non-woven electrospun fibrous matrices since it is already established that mechanical data depend not only on the type of polymeric material and on single fiber features, but also on the arrangement of the fibers in the nonwoven matrices, such as fiber direction in the mat, fiber curvature, fiber interconnections, fiber fusion at contact points and mat porosity $[25,26]$. Elastic modulus $(E)$, stress at break $\left(\sigma_{b}\right)$ and elongation at break $\left(\varepsilon_{b}\right)$ of PBS, PBS/PBTDG, P(BS $\left.{ }_{10} B T D G_{10}\right)$, PBS/PBDG, and $P\left(B S_{11} B D G_{11}\right)$ are reported in Figure 2. Both the two blends and the copolymers were characterized by a lower elastic modulus than PBS and by a higher elongation at break (with the only exception of PBS/PBDG). Moreover, E and $\varepsilon_{b}$ were respectively lower and higher in the case of block copolymers compared to the corresponding blends. This behaviour could be due to the decrease in the amount of hard crystal phase when comparing the blend with the corresponding copolymer (Table 1). The presence of thioether bonds along the polymer backbone caused an increase of the elongation at 
break and a decrease of the elastic modulus compared to the polymers containing ether linkages with the same molecular architecture. This result could be ascribed to the higher chain flexibility of BTDG units due to the presence along the polymeric chain of C-S bonds longer than the $\mathrm{C}-\mathrm{O}$ ones and due to weaker interchain interactions because of the lower electronegativity of sulphur atoms compared to oxygen ones.
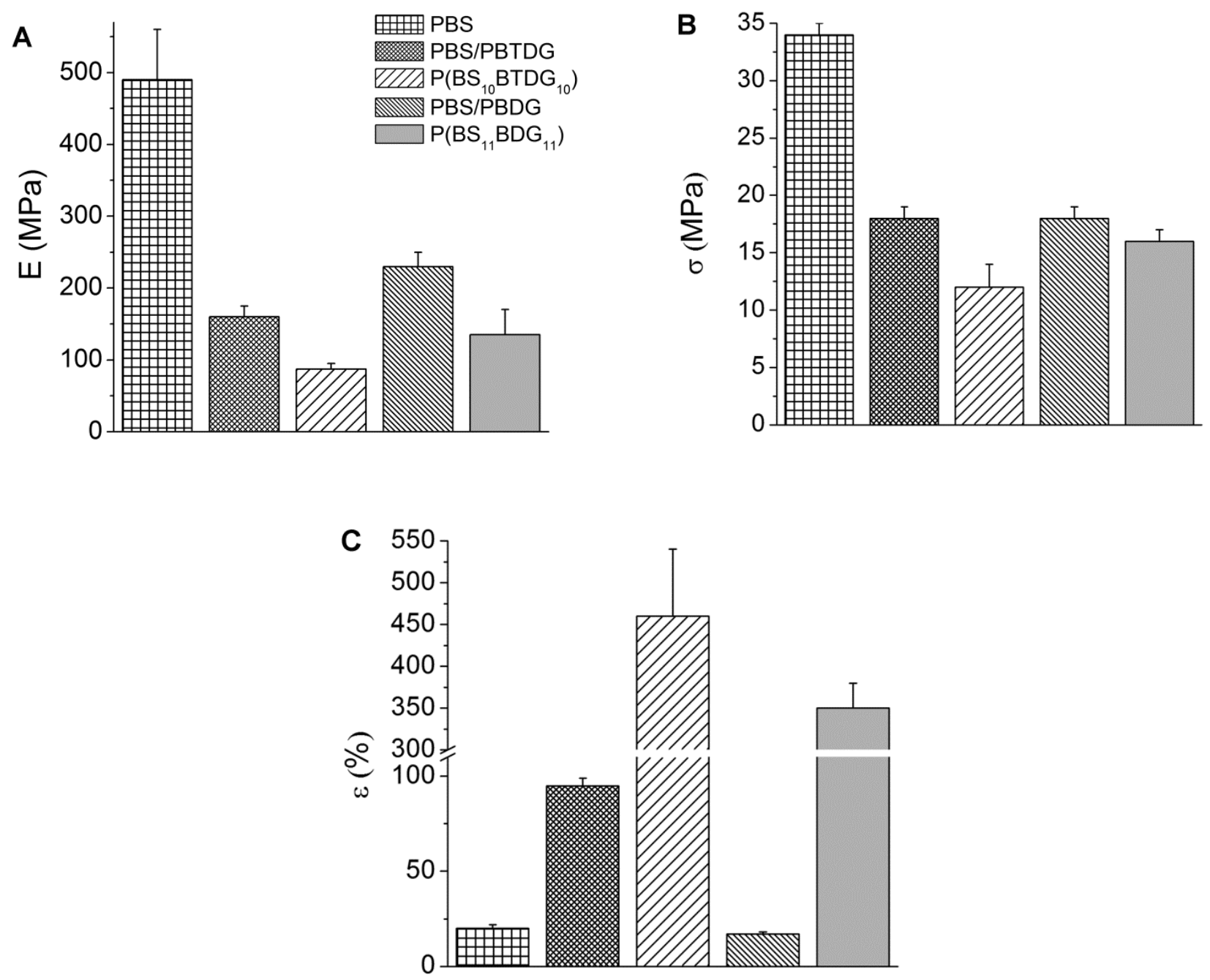

Figure 2. (A) Elastic modulus $E$; (B) stress at break $\sigma_{b}$ and $(C)$ deformation to break $\varepsilon_{b}$ of the polymers under investigation.

\subsubsection{Hydrolytic degradation}

Hydrolytic degradation experiments were performed under physiological conditions on the electrospun mats in order to evaluate the effect of the following factors on the hydrolysis rate: (i) introduction of hydrophilic BDG and BTDG co-units in the PBS macromolecular chain; (ii) type of heteroatom (O or $\mathrm{S}$ ) introduced; (iii) different molecular architecture 
(blend or block copolymer). Hydrolytic degradation of these scaffolds can be interpreted on the basis of two different factors: crystallinity degree and surface hydrophilicity [27].
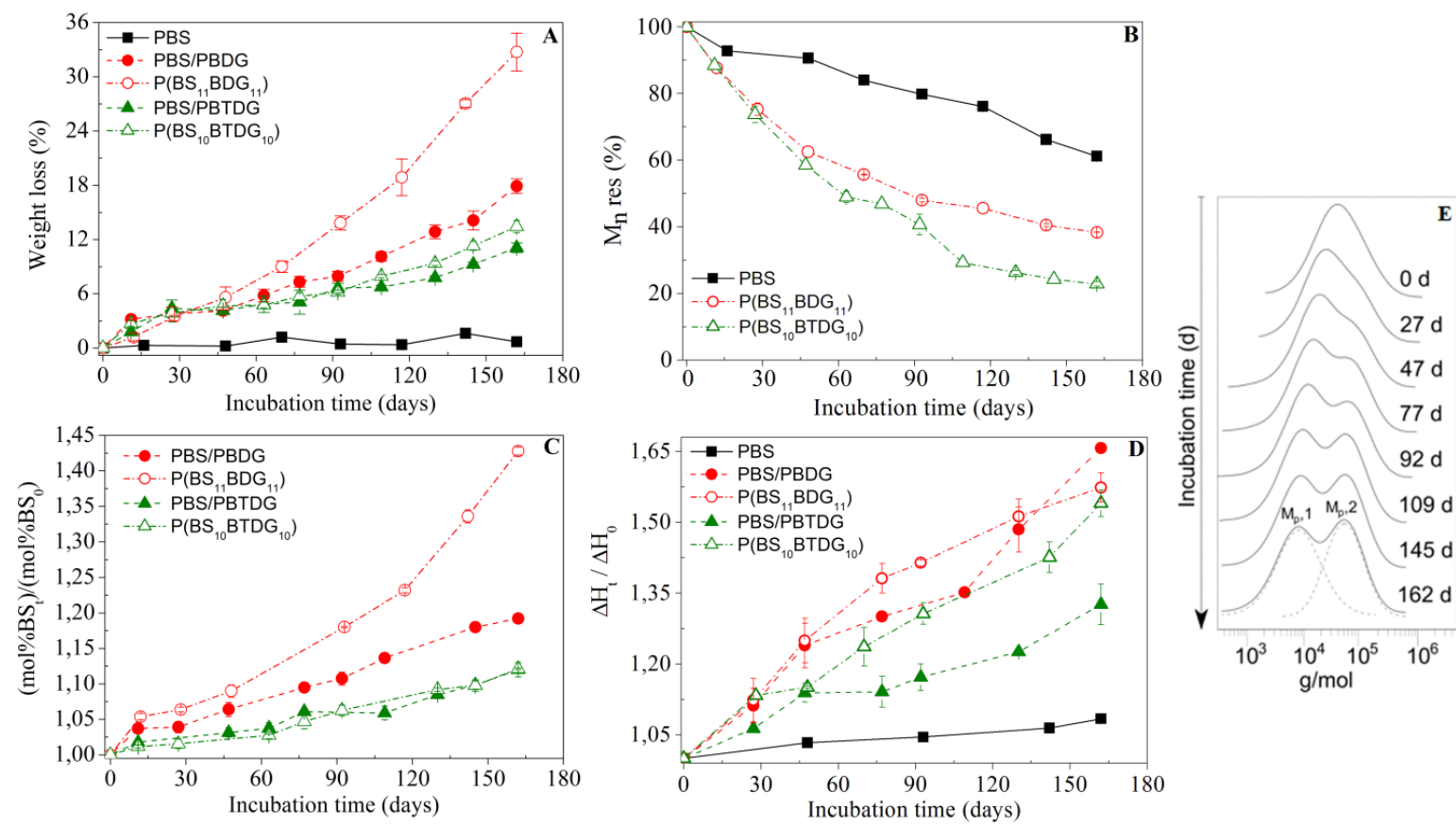

Figure 3. Hydrolytic degradation of polymeric scaffolds under investigation as a function of incubation time. (A) percentage weight loss; (B) percentage residual number molecular weight; (C) increase in BS mol\% content; (D) normalized heat of fusion; (E) GPC traces of PBS/PBDG blend as a function of incubation time.

Comparable results were obtained for both $P\left(B S_{11} B D G_{11}\right)-m$ and $P\left(B S_{11} B D G_{11}\right)-n$ indicating that the fiber diameters considered in the present work did not affect degradation rate. Therefore, for this polymer, average results of the two mats were reported (Figure 3). After 162 days of incubation, PBS practically did not lose weight whereas polymer blends and block copolymers appreciably degraded. In particular, etheroxygen containing polyesters showed a higher degradation rate with respect to sulphur containing ones. Moreover, Figure 3A shows that the weight loss of block copolymer is higher than the corresponding blend. The trend was particularly evident if we consider $P B S / P B D G$ and $P\left(B S_{11} B D G_{11}\right)$. On the contrary, only a small difference has been recorded in the case of PBS/PBTDG and $P\left(B S_{10} B T D G_{10}\right)$.

Overall, copolymers containing BDG sequences degraded faster than those containing BTDG ones because of the higher hydrophilicity of the former (Table 1). By comparing the blend with the corresponding copolymer, $\mathrm{P}\left(\mathrm{BS}_{11} \mathrm{BDG}_{11}\right)$ degraded to a higher extent than PBS/PBDG because of the lower crystallinity degree, while the two sulphur containing polymers underwent a similar degradation profile because of their comparable 
crystallinity degree and hydrophilicity (Table 1). The percentage of residual number average molecular weight for $P B S, P\left(B S_{11} B D G_{11}\right)$ and $P\left(B S_{10} B T D G_{10}\right)\left(M_{n}\right.$ res\%) is reported in Figure $3 \mathrm{~B}$ as a function of incubation time. The three samples underwent a decrease of $\mathrm{M}_{n}$ with time, although PBS did not show any weight loss in the time range investigated (Figure 3A). This is not surprising, taking into account that in the first stages of hydrolytic degradation a substantial decrease in molecular weight occurs even if weight losses are still negligible[28]. PBS decrease of $M_{n}$ was lower due to its high crystallinity degree, high crystal perfection and hydrophobicity, while in the case of copolymers the change of molecular weight was more evident and seemed to be affected by the type of polymer investigated: for $P\left(B S_{11} B_{D G} G_{11}\right)$ the $M_{n}$ decrease was higher than that of $\mathrm{P}\left(\mathrm{BS}_{10} \mathrm{BTDG}_{10}\right)$. The calculation of $\mathrm{Mn}_{\mathrm{n}}$ was not possible for the two blends, because the GPC traces of the analysed polymers revealed a shoulder since the very beginning of the incubation period that turned into two partially overlapped peaks. With increasing of the incubation time, a second peak was detectable (Figure 3E). Therefore, to monitor the degradation process, the molecular weight of both peaks $\left(M_{p}, 1\right.$ and $\left.M_{p}, 2\right)$ was calculated (Table S2). For both blends the position of one peak slightly changed $\left(M_{p}, 2\right)$, while $M_{p}, 1$ remarkably decreased with the increase of incubation time. By considering the difference in repeating units hydrophilicity, we can reasonably assume that $M_{p}, 1$ is related to either PBDG or PBTDG macromolecular chains that underwent a substantial hydrolytic degradation, while $\mathrm{Mp}_{\mathrm{p}} 2$ corresponds to PBS macromolecular chains, whose molecular weight decrease was significantly lower.

In order to gain a better understanding of the hydrolysis mechanism occurring in the polymers, ${ }^{1} \mathrm{H}-\mathrm{NMR}$ measurements were performed on degraded retrieved samples. Figure $3 \mathrm{C}$ reports the content of $\mathrm{BS}$ units in mol\%, at a certain time of degradation, normalized to the initial mol\% BS (mol\%BSt/mol\%BSo) as a function of degradation time. In all samples, an evident increase of BS content and a consequent decrease in the BDG and BTDG content was observed during degradation. The polymeric systems containing ether linkages (PBS/PBDG and $P\left(B_{11} B_{1} G_{11}\right)$ ) underwent a significant change of molar composition, more evident in the blend compared to the copolymer. On the other hand, PBS/PBTDG and P(BS $\left.{ }_{10} B D_{D G}\right)$, underwent a similar decrease of BTDG content. By comparing weight loss (Figure $3 \mathrm{~A}$ ) and chemical composition of the retrieved samples (Figure $3 \mathrm{C}$ ), it is evident that samples undergoing higher weight loss concomitantly displayed a higher increase of BS units. Therefore, the weight loss was mainly due to the solubilisation of either BDG or BTDG chain fragments since ester cleavage preferentially 
occurred on heteroatom-containing chain segments, which were easily solubilized in water due to their hydrophilic nature. Moreover, as expected, ether-oxygen-containing sequences demonstrated higher solubility in water with respect to sulphur-containing ones: the lower decrease in molecular weight and the contemporary higher weight loss of $P\left(B S_{11} B D G_{11}\right)$ with respect to $P\left(B S_{10} B T D G_{10}\right)$, indicated that longer $B D G$ sequences were solubilized in water generating a more pronounced change in $\mathrm{BS} \mathrm{mol} \%$.

A confirmation that the amorphous regions of a polymer degraded more quickly than the crystalline ones was obtained by analysing the degraded samples with DSC. All the calorimetric traces were found to be characterized by an endothermic peak associated with the fusion process of the crystalline portion of the material (similar to Figure S1). The corresponding heat of fusion, at a certain time of degradation, was normalized to the heat of fusion of the corresponding non-degraded sample $\left(\Delta \mathrm{H}_{\mathrm{t}} / \Delta \mathrm{H}_{0}\right)$. In all the polymeric compositions, the normalized heat of fusion regularly increased with incubation time, with the exception of PBS, whose crystallinity degree was not significantly affected (Figure 3D). It is worth remembering that the increment of the crystalline/amorphous ratio can be also due to annealing, which occurs at a temperature comprised between $T_{g}$ and $T_{m}$ (i.e. $37^{\circ} \mathrm{C}$ in our case). The increase of $\Delta \mathrm{H}_{t} / \Delta \mathrm{H}_{0}$ can, therefore, be considered as the sum of two contributions: (i) the higher degradation of the amorphous region with respect to crystalline one and (ii) annealing. In block copolymers, the annealing process plays a greater role than in the blends because of the lower crystallization rate of the former, as previously reported[12, 13]. As a matter of fact, the crystallization process becomes more difficult as the BS block became shorter due to the effect of the second block (either BDG or BTDG), which limited the transport of the BS blocks on the crystal surface and acted as defect during chain folding. In this respect, the more pronounced increase in the heat of fusion of $P\left(B S_{10} B T D G_{10}\right)$ compared to PBS/PBTDG is not surprising, although they showed a comparable weight loss (Figure $3 \mathrm{~A}$ ). From a comparison between etheroxygen-containing polymers and sulphur-containing ones, the higher increase in the heat of fusion of the former can be explained on the basis of their higher degradation rate.

\subsubsection{Seeding hMSCs on scaffolds}

To examine the performance of these new polyesters for skeletal tissue engineering, hMSCs were cultured on the samples for 28 days in BM, OM, CM, or MM. BM is a maintenance medium that does not trigger the osteogenic and chondrogenic differentiation of hMSCs, while CM is able to support the chondrogenic differentiation of 
hMSCs $[29,30] . \mathrm{OM}$ is a medium that supports the osteogenic differentiation of hMSCs and $\mathrm{MM}$ is a medium that also promotes the formation of a mineralized matrix[30, 31]. The bioactivity of hMSCs was assessed by monitoring the GAG production, cellular morphology, cellular metabolism, ALP activity, and relative osteogenic and chondrogenic gene expressions. Polystyrene tissue culture plates were used as a positive control (data not shown). To investigate for a possible influence of fiber diameter, scaffolds of the same chemistry but different fiber diameter were assessed between $\mathrm{P}\left(\mathrm{BS}_{11} \mathrm{BDG}_{11}\right)-\mathrm{n}(430 \pm$ $260 \mathrm{~nm})$ and $P\left(B S_{11} B D G_{11}\right)-m(890 \pm 410 \mathrm{~nm})$. To analyze the effect of the introduction of a co-monomer (either BDG or BTDG) in the PBS chain, scaffolds of similar fiber dimension but different chemical group were examined among PBS, $P\left(B S_{10} B T D G_{10}\right)$ and $P\left(B S_{11} B D G_{11}\right)-n$. To investigate the effect of reactive blending, PBS/PBTDG and PBS/PBDG scaffolds were considered. Finally, to evaluate the effect of blending versus copolymerization, $P B S / P B D G$ blend and $P\left(B_{11} B_{D G} G_{11}\right)-m$, which had a comparable fiber dimension, were considered.

\subsubsection{Cell morphology and metabolic activity}

Cell attachment and morphology on scaffolds were examined by SEM (Figure S2, Figure S3 and Figure S4). After 28 days culture, it could be seen that the hMSCs migrated, attached and proliferated on all types of scaffolds in all investigated media. No significant difference in cell morphology was observed between $P\left(B S_{11} B D G_{11}\right)-n$ and $P\left(B S_{11} B D G_{11}\right)-$ $\mathrm{m}$ in all media, indicating that the fiber dimension considered in the present work did not affect cell morphology. In OM, hMSCs on PBS/PBTDG and P(BS 10BTDG $\left._{10}\right)$ had a more spread morphology compared to $P B S / P B D G$ and $P\left(B S_{11} B D G_{11}\right)-n$ respectively, therefore revealing an effect of BTDG or BDG presence on cell morphology. However, in CM, hMSCs displayed morphology with more bi-polar or multipolar extensions on PBS/PBTDG scaffolds in comparison with PBS/PBDG. In addition, no difference in cell morphology was observed between PBS/PBDG blend and its corresponding block copolymer of $\mathrm{P}\left(\mathrm{BS}_{11} \mathrm{BDG}_{11}\right)$ in all media.

Cell metabolic activity on seeded scaffolds was evaluated at days 7 and 28 using a Presto Blue assay (Figure S5). Metabolic activity, which is indirectly related to cell viability, increased over the cell culture period in all media for all scaffolds. $P\left(B S_{11} B D G_{11}\right)-n$ and $P\left(B S_{11} B D G_{11}\right)-m$ had a similar metabolic activity cultured in both $B M$ and $C M$ through the culture time points. In $\mathrm{OM}, \mathrm{P}\left(\mathrm{BS}_{11} \mathrm{BDG}_{11}\right)-\mathrm{n}$ showed a significantly higher metabolic activity at day 28 compared with $P\left(B S_{11} B D G_{11}\right)-m$ due to their difference in fiber 
dimension. PBS constructs displayed a slightly higher metabolic activity compared to $P\left(B S_{10} B T D G_{10}\right)$ in both $B M$ and $C M$ at day 28 due to the presence of co-units of BTDG. Similarly, the presence of BDG blocks along polymer chain did not enhance the cell metabolism in comparison with PBS under the same conditions. Interestingly, in the copolymers the introduction of BDG blocks generated a higher cell metabolism in $\mathrm{CM}$ compared to BTDG blocks, while in OM PBS/PBDG displayed higher cell metabolic activity compared to PBS/PBTDG.

\subsubsection{ALP activity}

To investigate early osteogenic differentiation of hMSCs cultured onto different scaffolds either in BM or in OM, we performed quantitative analysis of ALP at days 7 and 28 (Figure $4 \mathrm{~A}$ and $4 \mathrm{~B}$ ). ALP is commonly known as an early marker of osteogenic differentiation, which is involved in the formation of calcium phosphate crystals [32]. The ALP activity increased during cell culture in $B M$ and $O M$ and showed no remarkable difference between scaffolds at day 7 . After 28 days, $P\left(B S_{11} B D G_{11}\right)$-m showed a similar ALP activity as $P\left(B S_{11} B D G_{11}\right)-n$ in OM. Shih et al. grew human $h M S C$ s on electrospun type I collagen nanofibers[33]. They demonstrated that no significant difference of ALP activity of hMSCs after 12 days of culture in OM was observed between scaffolds with fiber diameter of 200-500 nm and fiber diameter of 500-1,000 nm. PBS, $P\left(B S_{10} B_{T D G_{10}}\right)$ and $P\left(B S_{11} B D G_{11}\right)-n$ scaffolds supported a similar $A L P$ activity in both $B M$ and $O M$, which indicated that the presence of block units of BTDG and BDG do not elicit a specific effect on ALP activity. We also observed that ALP activity in both BM and OM was comparable for both PBS/PBTDG and PBS/PBDG. This suggested that the introduction of a second component of the blend, being either PBTDG or PBDG, has a similar effect on ALP activity. It is worth mentioning that the ALP activity on $P\left(B S_{11} B D G_{11}\right)-m$ was higher than the corresponding blend in both investigated media.

The results of ALP activity were further confirmed by staining cells with ALP (Figure S6 and S7). Low levels of ALP staining were present on all types of scaffolds at day 7 in both media without significant difference. $P\left(B S_{11} B D G_{11}\right)-n$ showed similar density in ALP staining during the cell culture period in all media compared to $P\left(B S_{11} B D G_{11}\right)-m$. At day 28 , the intensity of $A L P$ staining remarkably increased on $P B S$ and $P\left(B S_{10} B T D G_{10}\right)$ scaffolds in $B M$ compared to $P\left(B S_{11} B D G_{11}\right)-n$, suggesting a possible inductive effect on stem cell early osteogenic differentiation of these two chemical compositions. Furthermore, ALP staining increased in intensity on PBS, $P\left(B S_{10} T D G_{10}\right)$, and 
$\mathrm{P}\left(\mathrm{BS}_{11} \mathrm{BDG}_{11}\right)-\mathrm{n}$ scaffolds in OM. Interestingly, PBS/PBDG and PBS/PBTDG scaffolds exhibited a constantly lower ALP activity at both time point in all media. In addition, $\mathrm{P}\left(\mathrm{BS}_{11} \mathrm{BDG}_{11}\right)-\mathrm{m}$ showed a higher ALP staining in OM than the corresponding blend.

A

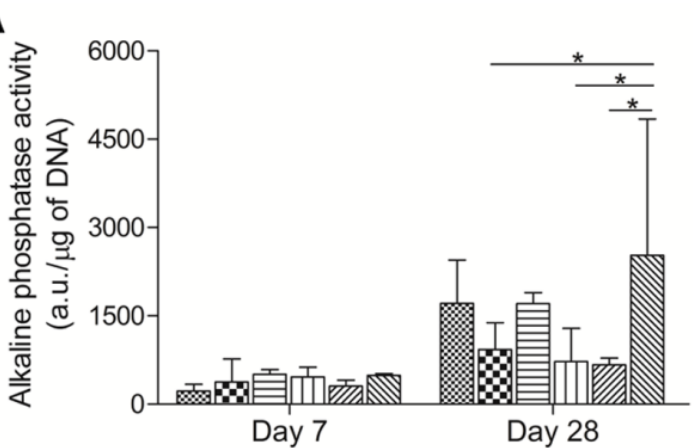

C

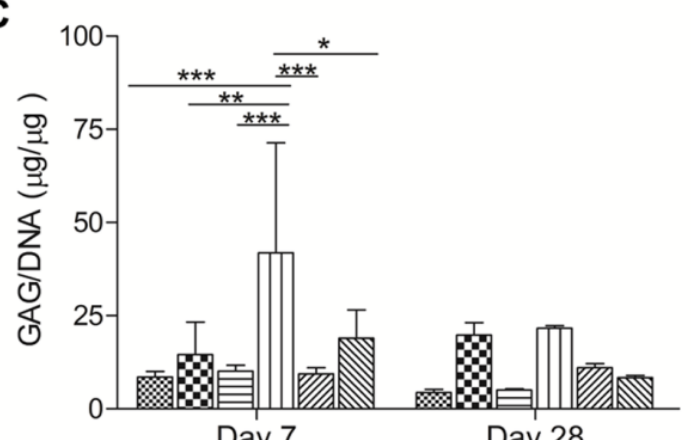

B

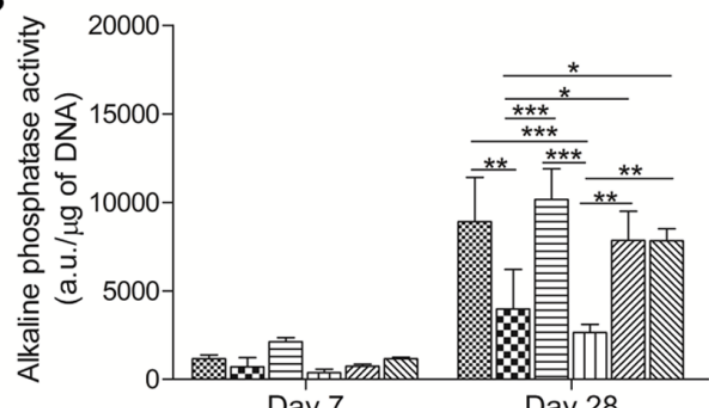

D

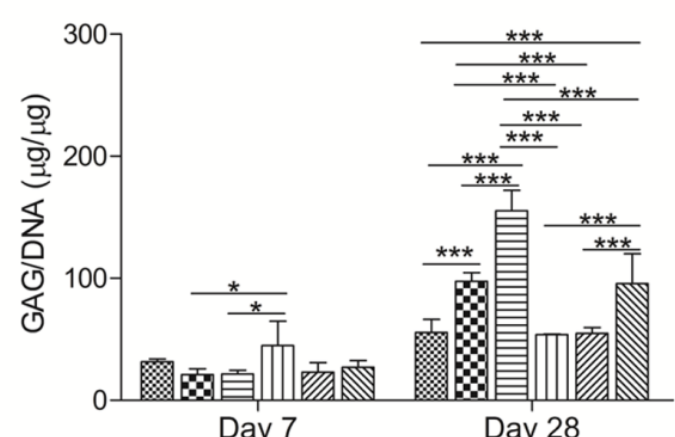

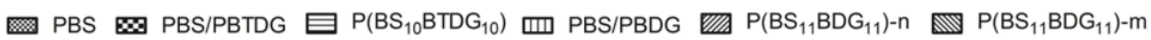

Figure 4. ALP activity of hMSCs cultured on scaffolds in $B M(A)$ and $O M(B)$ at days 7 and 28. The final results of ALP activity level was normalized by DNA amount. GAG production of hMSCs cultured on scaffolds in BM (C) and CM (D) at day 7 and day $28 .{ }^{*} p<0.05,{ }^{* *} p<0.01,{ }^{* * *} p<0.001$.

\subsubsection{GAG assay}

The quantification of GAG synthesis is shown in Figure 4C and 4D. No significant differences were found between $P\left(B S_{11} B D G_{11}\right)-m$ and $P\left(B S_{11} B D G_{11}\right)-n$ in $B M$. However, after 28 days, $P\left(B S_{11} B D G_{11}\right)-m$ had a significant higher $G A G$ deposition than $P\left(B S_{11} B D G_{11}\right)-n$ in $C M$, due to their difference in fiber dimension. This results is consistent with the findings by Bean et al.[34], who demonstrated that seeding hMSCs onto microfibers at a higher cell density promotes GAG production better than nanofibers. In $\mathrm{CM}$, at day $28, \mathrm{P}\left(\mathrm{BS}_{10} \mathrm{BTDG}_{10}\right)$ scaffold presented a significant increase in $\mathrm{GAG}$ production compared to $\mathrm{PBS}$ and $\mathrm{P}\left(\mathrm{BS}_{11} \mathrm{BDG}_{11}\right)-\mathrm{n}$. In addition, PBS/PBTDG displayed a significantly higher GAG amount than PBS/PBDG in CM. Both these results may indicate that the systems containing BTDG units may have a better effect on production of GAG in $\mathrm{CM}$ with respect to those containing BDG units. This result could be due to the presence 
of sulphur atoms along the polymer chain which are always observed in all of GAG[35]. $\mathrm{P}\left(\mathrm{BS}_{11} \mathrm{BDG}_{11}\right)$ - $\mathrm{m}$ highlighted a lower $\mathrm{GAG}$ production than the corresponding blend in all media, which is in contrast to ALP activity. A recent study showed that hMSCs cultured on PCL electrospun scaffolds cultured for 4 weeks in chondrogenic medium had a GAG/DNA concentration of about $15 \mu \mathrm{g} / \mu \mathrm{g}[36]$. Compared to this study, our scaffolds showed a higher GAG production at the same time point, However, in doing such comparison with other scaffolds used in the literature, we may still be limited by the potentially different cell donors and related intrinsic variability between patients.

\subsubsection{The mineralization of $h M S C s$}

ARS has been used to examine the level of calcium deposition after 28 days (Figure 5). There were no apparently differences in ARS between scaffolds in BM. The intensity of ARS onto $P\left(B S_{11} B D G_{11}\right)-n$ scaffolds was higher than both $P\left(B S_{10} B T D G_{10}\right)$ and $P B S$ scaffolds and no significant differences were found between $P\left(B S_{10} B T D G_{10}\right)$ and $P B S$ in MM. These results suggested that the introduction of BDG blocks supported better mineralization when compared to the introduction of BTDG blocks. No appreciable differences in ARS were observed between PBS/PBTDG and PBS/PBDG, as well as between $P B S / P B D G$ and $P\left(B S_{11} B D G_{11}\right)-m$ for all media. Xuejun et al. determined the cell viability, growth and differentiation of hMSCs in PLGA (PLA: PGA ratio of 85:15) nanofibers [37]. They demonstrated that hMSCs treated with osteogenic medium showed positive staining to alizarin red after 4 weeks. The intensity of alizarin red staining was lower than that of our scaffolds. However, as explained earlier for chondrogenic studies, different cell donors were used, which should be taken into consideration when comparing our results with the literature. 

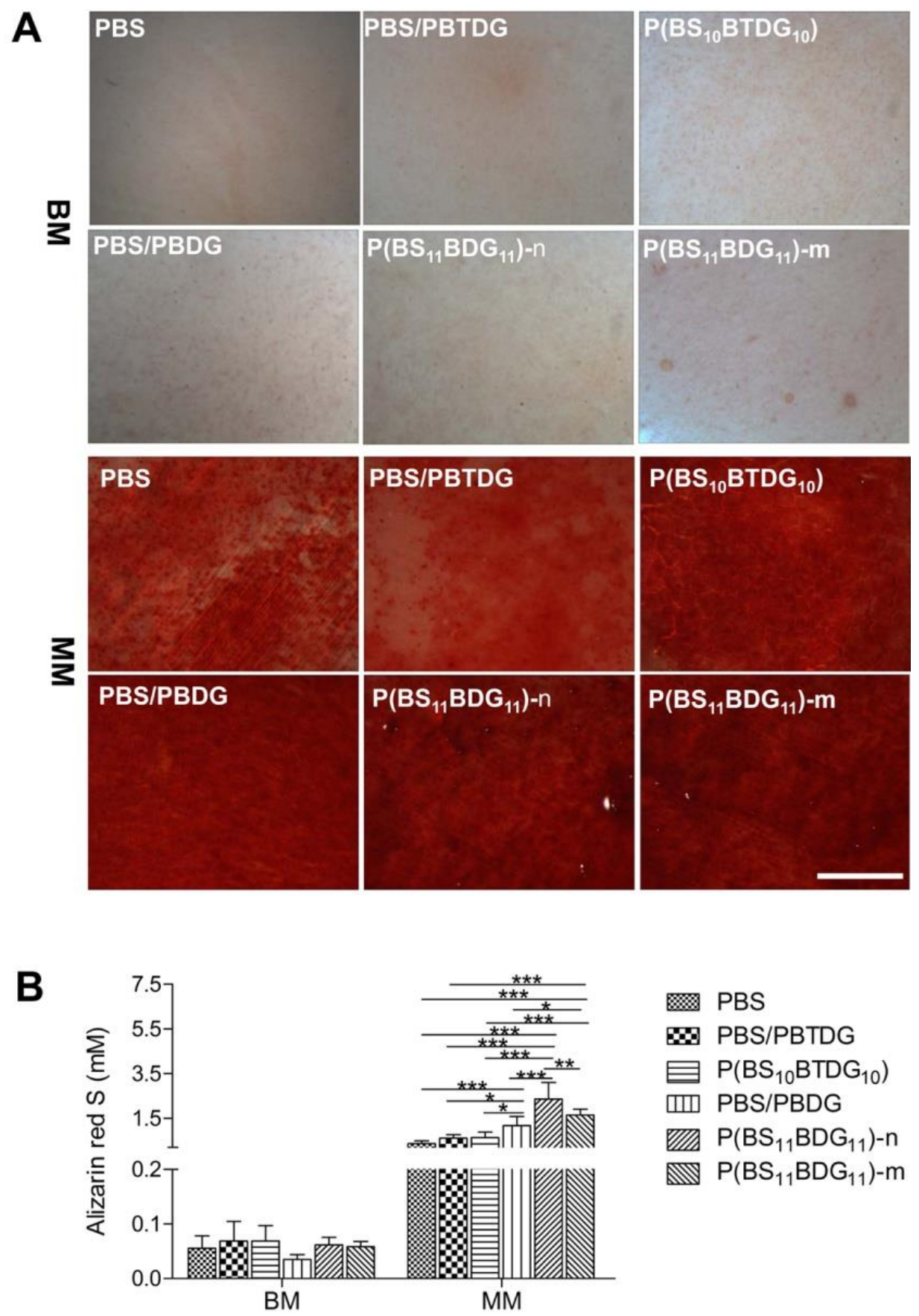

Figure 5. Mineralization of hMSCs on scaffolds was investigated after 28 days of culture in BM and MM. The production of calcium was stained with ARS (A), Scale bar $=1 \mathrm{~mm}$. (B) Quantification of the calcium deposition per scaffold by ARS assay at day 28. ${ }^{*} \mathrm{p}<0.05,{ }^{* *} \mathrm{p}<0.01,{ }^{* * *} \mathrm{p}<0.001$

\subsubsection{Osteogenic gene expression}

The investigation of osteoblast-related gene expression for hMSCs on scaffolds was performed at day 7 and day 28 (Figure 6). In both media investigated, $P\left(B S_{11} B_{D} G_{11}\right)-n$ and $P\left(B S_{11} B D G_{11}\right)-m$ showed a similar osteogenic gene expression including $A L P$, Runtrelated transcription factor 2(RUNX2), Collagen type I alpha 1 (COL1A1) and Bone 
sialoprotein (BSP), thus confirming that the difference in fiber dimension in the present work did not affect osteogenic differentiation. ALP expression for PBS, $P\left(B S_{10} T_{D G_{10}}\right)$ and $P\left(B S_{11} B D G_{11}\right)-n$ scaffolds were almost the same in BM. However, ALP expression on PBS was higher than on $P\left(B S_{11} B D G_{11}\right)$-n scaffolds at day 28 in OM. RUNX2 strongly affects hMSCs osteogenic differentiation in the early stage, resulting in enhanced ALP, osteocalcin (OCN), osteopontin (OPN), and BSP expression [38]. The expression of RUNX2 on PBS was higher than that on $P\left(B S_{11} B D G_{11}\right)-n$ scaffolds at both time points in $B M . P\left(B S_{10} B T D G_{10}\right)$ displayed a significant higher expression of RUNX2 at day 7 in $B M$ in comparison with $P\left(B S_{11} B D G_{11}\right)-n$. COL1A1 is associated with the formation of the $E C M$, being considered essential for the development of an osteoblastic phenotype[39]. PBS showed a significant higher COL1A1 expression than $P\left(B S_{11} B D G_{11}\right)-n$ after 28 days in $\mathrm{BM}$, although no significant differences were found in OM. BSP, a late marker of hMSCs osteogenic differentiation, is a highly sulphated and glycosylated phosphoprotein in bone matrix [40]. BSP expression on PBS was significantly higher than that on $P\left(B S_{11} B B_{11}\right)-n$ at day 28 in OM. All gene expression tested did not highlight significant differences between PBS/PBDG and $P\left(B S_{11} B D G_{11}\right)-m$. These results suggest that PBS scaffolds may elicit a higher early osteogenic differentiation than copolymers containing BDG or BTDG units.
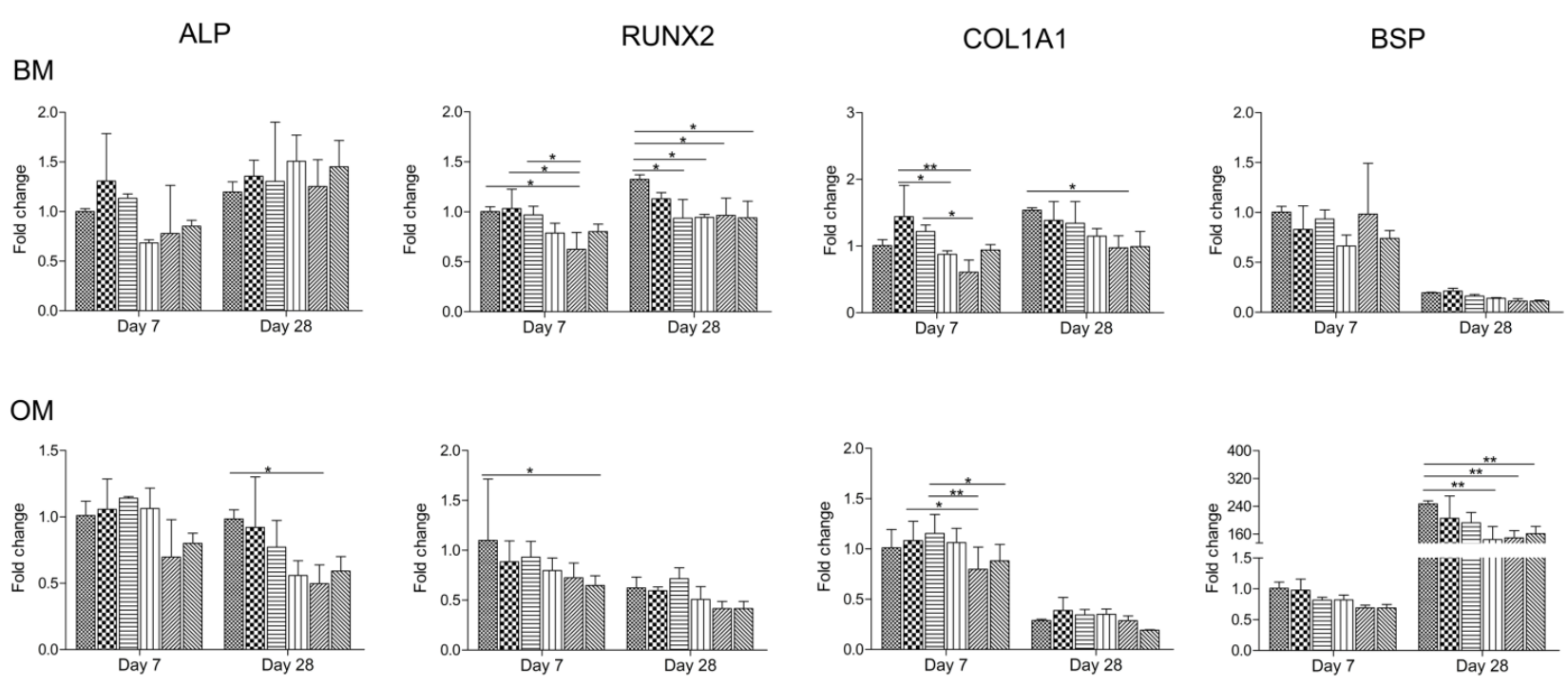

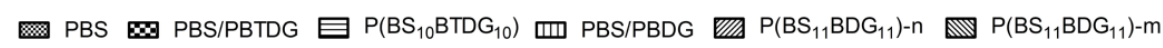

Figure 6. Quantitative RT-PCR gene expression analysis of osteoblast-related genes in BM and OM at days7 and 28. ${ }^{*} \mathrm{p}<0.05,{ }^{* *} \mathrm{p}<0.01$. 


\subsubsection{Chondrogenic gene expression}

To know the potential of chondrogenic differentiation of hMSCs on the scaffolds under investigation, we evaluated a panel of chondrocyte-related genes: collagen, type II alpha 1 (COL2A1), SOX9, aggrecan (ACAN) and activated leukocyte cell adhesion molecule (ALCAM) (Figure 7). No significant difference in expression of the investigated gene profiles was observed between $P\left(B S_{11} B D G_{11}\right)-n$ and $P\left(B S_{11} B D G_{11}\right)-m$ despite of their difference in fiber dimension. COL2A1 is a specific marker of hyaline cartilage ECM[41]. $\mathrm{P}\left(\mathrm{BS}_{11} \mathrm{BDG}_{11}\right)$ and $\mathrm{P}\left(\mathrm{BS}_{10} \mathrm{BTDG_{10 }}\right)$ scaffolds displayed a higher expression of COL2A1 than PBS scaffolds at day 28 in BM. It could be inferred that the introduction of BTDG and BDG blocks along the PBS macromolecular chain was favourable for COL2A1 expression. The expression of COL2A1 on PBS/PBTDG scaffold was consistently higher than that on PBS/PBDG scaffolds, indicating that the blend containing BTDG units better supported COL2A1 expression compared to BDG units. SOX9, an early marker for chondrogenesis, is the transcriptional activator of COL2A1, COL9A1 and ACAN[42, 43]. The expression of SOX9 was similar for all scaffolds at both time points in BM, while the expression of this gene significantly increased onto PBS/PBTDG scaffold at day 28 in CM. ALCAM is expressed on human articular chondrocytes and function as a marker for stemness[44]. At day 28, PBS/PBTDG scaffolds supported a significant higher expression of ALCAM in both BM and CM compared to PBS/PBDG scaffolds, thus suggesting that the blend containing BTDG units better supports ALCAM expression compared to BDG units. ACAN is one of the main proteoglycan in the articular cartilage matrix [45]. In BM, $A C A N$ on $P\left(B S_{10} T D G_{10}\right)$ scaffold showed a significant up-regulation compared to PBS, due to the presence of BTDG units along the polymer chain. A significant higher expression of ACAN was observed on PBS/PBTDG scaffold compared to that on PBS/PBDG in both condition media at day 28. Together, gene expressions results indicated that BTDG units displayed better support of hMSCs chondrogenesis with respect to BDG units. This result could be ascribed to the presence of sulphur atoms in the BTDG units. It is also worth noticing that PBS/PBTDG blends showed better performances than the corresponding block copolymer. 

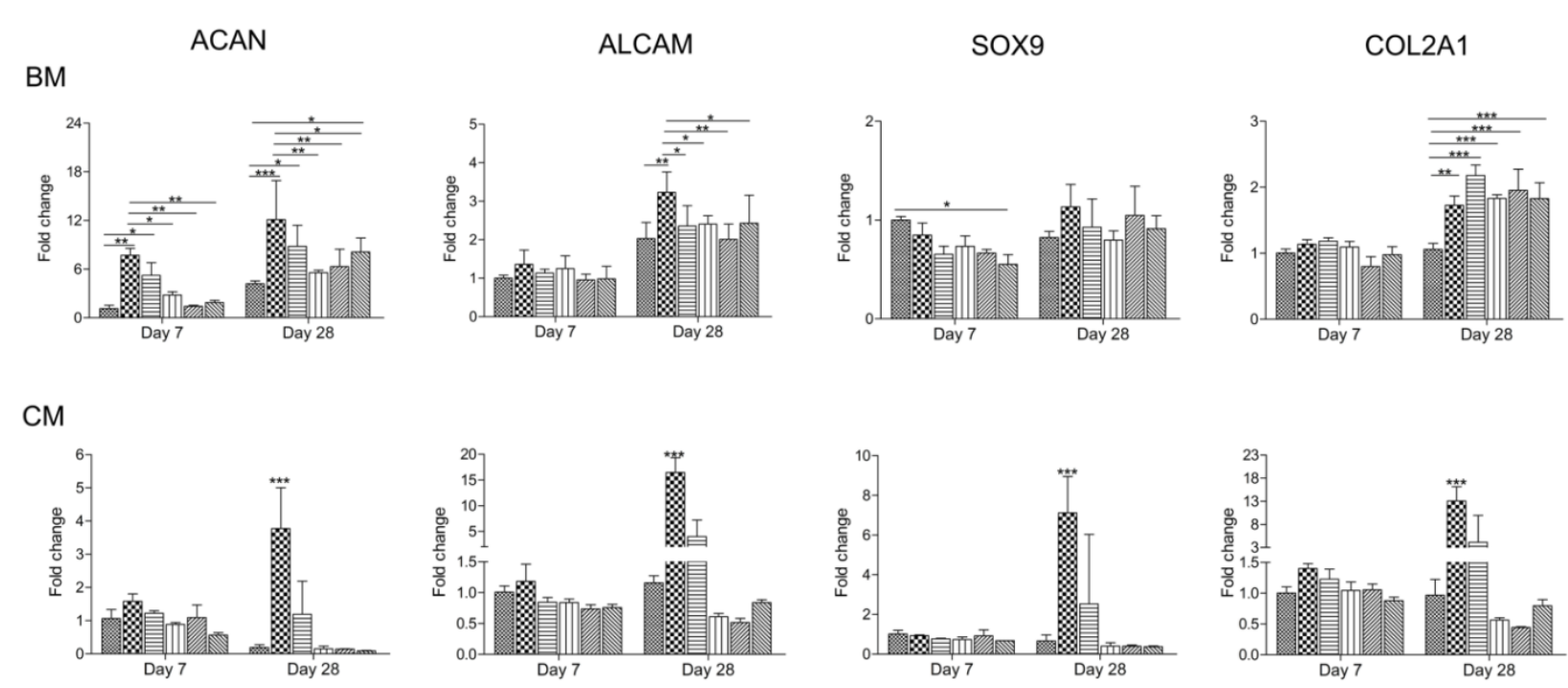

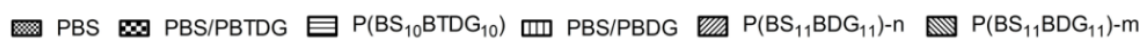

Figure 7. Quantitative RT-PCR gene expression analysis of chondrogenisis-related genes in BM and CM at days 7 and $28 .{ }^{*} p<0.05,{ }^{* *} p<0.01,{ }^{* * *} p<0.001$.

\subsection{Conclusions}

In the present study, PBS-based scaffolds from block copolymers containing heteroatoms have been successfully prepared by electrospinning. Hydrolytic degradation studies under physiological conditions showed that the hydrolysis rate can be tuned by acting on the kind of heteroatom introduced and on the molecular architecture, as these factors deeply influence both the crystallinity and the surface wettability of the investigated materials. The presence of BTDG units in the copolymers demonstrated a better support of chondrogenic differentiation of hMSCs compared to the presence of BDG units. Additionally, PBS scaffolds induced enhanced early osteogenic differentiation of hMSCs and the additional presence of ether units (BDG) resulted in enhanced mineralization. It is also worth mentioning that the same chemical composition, but a different molecular architecture (i.e. blend or block copolymer) highlighted a very different effect on hMSCs cell function such as differentiation. Therefore, molecular architecture revealed as an important parameter in terms of modulating the chemistry of scaffold. Taken together, these results demonstrated that the new copolymer family may therefore find applications for the regeneration of skeletal tissues in regenerative medicine field. In the present work, we primarily focused on understanding cell-materials interactions in these new polymeric scaffolds, which is a fundamental required step in biomaterial science before continuing with more translational in vivo studies. In this respect, in vivo studies will be planned for future translational work. 


\section{References}

[1] Chen H, Fan X, Xia J, Chen P, Zhou X, Huang J, et al. Electrospun chitosan-graft-poly ( $\varepsilon$ caprolactone)/poly ( $\varepsilon$-caprolactone) nanofibrous scaffolds for retinal tissue engineering. International journal of nanomedicine. 2011;6:453.

[2] Agarwal S, Wendorff $\mathrm{JH}$, Greiner A. Use of electrospinning technique for biomedical applications. Polymer. 2008;49:5603-21.

[3] Ulery BD, Nair LS, Laurencin CT. Biomedical applications of biodegradable polymers. J Polym Sci, Part B: Polym Phys. 2011;49:832-64.

[4] Holzapfel BM, Reichert JC, Schantz J-T, Gbureck U, Rackwitz L, Nöth U, et al. How smart do biomaterials need to be? A translational science and clinical point of view. Adv Drug Del Rev. 2013;65:581603.

[5] Bechthold I, Bretz K, Kabasci S, Kopitzky R, Springer A. Succinic acid: a new platform chemical for biobased polymers from renewable resources. Chemical engineering \& technology. 2008;31:647-54.

[6] M. Gigli, M. Fabbri, N. Lotti, R. Gamberini, B. Rimini, A. Munari. Poly(butylene succinate)-based polyesters for biomedical applications: a review Eur. Polym. J. 2015; Ref. No.: EUROPOL-D-15-00633.

[7] Soccio M, Lotti N, Gigli M, Finelli L, Gazzano M, Munari A. Reactive blending of poly (butylene succinate) and poly (triethylene succinate): characterization of the copolymers obtained. Polym Int. 2012;61:1163-9.

[8] Reilly GC, Engler AJ. Intrinsic extracellular matrix properties regulate stem cell differentiation. J Biomech. 2010;43:55-62.

[9] Faia-Torres AB, Guimond-Lischer S, Rottmar M, Charnley M, Goren T, Maniura-Weber K, et al. Differential regulation of osteogenic differentiation of stem cells on surface roughness gradients. Biomaterials. 2014;35:9023-32.

[10] Higuchi A, Ling Q-D, Chang Y, Hsu S-T, Umezawa A. Physical cues of biomaterials guide stem cell differentiation fate. Chem Rev. 2013;113:3297-328.

[11] Gigli M, Lotti N, Gazzano M, Finelli L, Munari A. Macromolecular design of novel sulfur-containing copolyesters with promising mechanical properties. J Appl Polym Sci. 2012;126:686-96.

[12] Gigli M, Lotti N, Gazzano M, Finelli L, Munari A. Synthesis and characterization of novel poly (butylene succinate)-based copolyesters designed as potential candidates for soft tissue engineering. Polymer Engineering \& Science. 2013;53:491-501.

[13] Soccio M, Lotti N, Gazzano M, Govoni M, Giordano E, Munari A. Molecular architecture and solid-state properties of novel biocompatible PBS-based copolyesters containing sulphur atoms. React Funct Polym. 2012;72:856-67.

[14] Gualandi C, Soccio M, Govoni M, Valente S, Lotti N, Munari A, et al. Poly (butylene/diethylene glycol succinate) multiblock copolyester as a candidate biomaterial for soft tissue engineering: Solid-state properties, degradability, and biocompatibility. J Bioact Compat Polym. 2012;27:244-64.

[15] Gualandi C, Soccio M, Saino E, Focarete ML, Lotti N, Munari A, et al. Easily synthesized novel biodegradable copolyesters with adjustable properties for biomedical applications. Soft Matter. 2012;8:5466-76.

[16] Gigli M, Govoni M, Lotti N, Giordano ED, Gazzano M, Munari A. Biocompatible multiblock aliphatic polyesters containing ether-linkages: influence of molecular architecture on solid-state properties and hydrolysis rate. RSC Advances. 2014;4:32965-76. 

and Thio-ether Linkages for Skeletal Differentiation of Human Mesenchymal Stromal Cells

[17] Gigli M, Lotti N, Vercellino M, Visai L, Munari A. Novel ether-linkages containing aliphatic copolyesters of poly (butylene 1, 4-cyclohexanedicarboxylate) as promising candidates for biomedical applications. Materials Science and Engineering: C. 2014;34:86-97.

[18] Sekiya I, Larson BL, Smith JR, Pochampally R, Cui JG, Prockop DJ. Expansion of human adult stem cells from bone marrow stroma: conditions that maximize the yields of early progenitors and evaluate their quality. Stem Cells. 2002;20:530-41.

[19] Smith JR, Pochampally R, Perry A, Hsu SC, Prockop DJ. Isolation of a highly clonogenic and multipotential subfraction of adult stem cells from bone marrow stroma. Stem Cells. 2004;22:823-31.

[20] Leferink A, Hendrikson W, Rouwkema J, Karperien M, Blitterswijk vC, Moroni L. Increased cell seeding efficiency in bioplotted three-dimensional PEOT/PBT scaffolds. J Tissue Eng Regen Med. 2013.

[21] Zhang J, Luo X, Barbieri D, Barradas AM, de Bruijn JD, Van Blitterswijk CA, et al. The size of surface microstructures as an osteogenic factor in calcium phosphate ceramics. Acta Biomater. 2014;10:3254-63.

[22] Gregory CA, Gunn WG, Peister A, Prockop DJ. An Alizarin red-based assay of mineralization by adherent cells in culture: comparison with cetylpyridinium chloride extraction. Anal Biochem. 2004;329:7784.

[23] Gaharwar AK, Mihaila SM, Swami A, Patel A, Sant S, Reis RL, et al. Bioactive silicate nanoplatelets for osteogenic differentiation of human mesenchymal stem cells. Adv Mater. 2013;25:3329-36.

[24] Gigli M, Negroni A, Zanaroli G, Lotti N, Fava F, Munari A. Environmentally friendly PBS-based copolyesters containing PEG-like subunit: Effect of block length on solid-state properties and enzymatic degradation. React Funct Polym. 2013;73:764-71.

[25] Rizvi M, Kumar P, Katti D, Pal A. Mathematical model of mechanical behavior of micro/nanofibrous materials designed for extracellular matrix substitutes. Acta Biomater. 2012;8:4111-22.

[26] Wei X, Xia Z, Wong S-C, Baji A. Modelling of mechanical properties of electrospun nanofibre network. International Journal of Experimental and Computational Biomechanics. 2009;1:45-57.

[27] Gigli M, Negroni A, Soccio M, Zanaroli G, Lotti N, Fava F, et al. Influence of chemical and architectural modifications on the enzymatic hydrolysis of poly (butylene succinate). Green Chemistry. 2012;14:2885-93.

[28] Mochizuki M, Hirami M. Structural effects on the biodegradation of aliphatic polyesters. Polym Adv Technol. 1997;8:203-9.

[29] Mentink A, Hulsman M, Groen N, Licht R, Dechering KJ, van der Stok J, et al. Predicting the therapeutic efficacy of MSC in bone tissue engineering using the molecular marker CADM1. Biomaterials. 2013;34:4592-601.

[30] Nandakumar A, Yang L, Habibovic P, van Blitterswijk C. Calcium phosphate coated electrospun fiber matrices as scaffolds for bone tissue engineering. Langmuir. 2009;26:7380-7.

[31] Nandakumar A, Barradas A, de Boer J, Moroni L, van Blitterswijk C, Habibovic P. Combining technologies to create bioactive hybrid scaffolds for bone tissue engineering. Biomatter. 2013;3.

[32] Liu W, Lipner J, Xie J, Manning CN, Thomopoulos S, Xia Y. Nanofiber scaffolds with gradients in mineral content for spatial control of osteogenesis. ACS applied materials \& interfaces. 2014;6:2842-9.

[33] Shih YRV, Chen CN, Tsai SW, Wang YJ, Lee OK. Growth of mesenchymal stem cells on electrospun type I collagen nanofibers. Stem Cells. 2006;24:2391-7. 
[34] Bean AC, Tuan RS. Fiber diameter and seeding density influence chondrogenic differentiation of mesenchymal stem cells seeded on electrospun poly ( $\varepsilon$-caprolactone) scaffolds. Biomedical Materials. 2015;10:015018.

[35] Heng BC, Cao T, Lee EH. Directing stem cell differentiation into the chondrogenic lineage in vitro. Stem Cells. 2004;22:1152-67.

[36] Ousema PH, Moutos FT, Estes BT, Caplan Al, Lennon DP, Guilak F, et al. The inhibition by interleukin 1 of MSC chondrogenesis and the development of biomechanical properties in biomimetic 3D woven PCL scaffolds. Biomaterials. 2012;33:8967-74.

[37] Xin X, Hussain M, Mao JJ. Continuing differentiation of human mesenchymal stem cells and induced chondrogenic and osteogenic lineages in electrospun PLGA nanofiber scaffold. Biomaterials. 2007;28:31625.

[38] Teplyuk NM, Galindo M, Teplyuk VI, Pratap J, Young DW, Lapointe D, et al. Runx2 regulates G protein-coupled signaling pathways to control growth of osteoblast progenitors. J Biol Chem. 2008;283:27585-97.

[39] Polini A, Pisignano D, Parodi M, Quarto R, Scaglione S. Osteoinduction of human mesenchymal stem cells by bioactive composite scaffolds without supplemental osteogenic growth factors. PLoS One. $2011 ; 6:$ e26211.

[40] Franceschi R. The developmental control of osteoblast-specific gene expression: role of specific transcription factors and the extracellular matrix environment. Crit Rev Oral Biol Medicine. 1999;10:40-57.

[41] Fayol D, Frasca G, Le Visage C, Gazeau F, Luciani N, Wilhelm C. Use of magnetic forces to promote stem cell aggregation during differentiation, and cartilage tissue modeling. Adv Mater. 2013;25:2611-6.

[42] Akiyama $\mathrm{H}$. Control of chondrogenesis by the transcription factor Sox9. Modern Rheumatology. 2008;18:213-9.

[43] Liu SQ, Tian Q, Hedrick JL, Hui JHP, Ee PLR, Yang YY. Biomimetic hydrogels for chondrogenic differentiation of human mesenchymal stem cells to neocartilage. Biomaterials. 2010;31:7298-307.

[44] Siegel G, Kluba T, Hermanutz-Klein U, Bieback K, Northoff H, Schäfer R. Phenotype, donor age and gender affect function of human bone marrow-derived mesenchymal stromal cells. BMC Med. 2013;11:146. [45] Kiani C, Liwen C, WU YJ, Albert JY, Burton BY. Structure and function of aggrecan. Cell Res. 2002;12:19-32. 


\section{Supporting Information}

Table S1. Electrospinning operating conditions.

\begin{tabular}{lccccc}
\hline Polymer & $\begin{array}{c}\text { solvent mixture } \\
(\mathrm{v}: \mathrm{v})\end{array}$ & $\begin{array}{c}\text { conc. } \\
(\mathrm{w} / \mathrm{v} \%)\end{array}$ & $\begin{array}{c}\text { voltage } \\
(\mathrm{kV})\end{array}$ & $\begin{array}{c}\text { flow rate } \\
(\mathrm{ml} / \mathrm{h})\end{array}$ & $\begin{array}{c}\mathrm{D}^{\mathrm{a}} \\
(\mathrm{cm})\end{array}$ \\
\hline PBS & DCM:2-CE $(80: 20)$ & 23 & 14 & 0.6 & 15 \\
PBS/PBDG & HFIP & 25 & 20 & 0.3 & 15 \\
P(BS ${ }_{11}$ BDG $\left._{11}\right)-\mathrm{m}^{\mathrm{b}}$ & DCM:2-CE $(90: 10)$ & 35 & 17 & 0.6 & 20 \\
P(BS BDDG $\left._{11}\right)-n^{\mathrm{c}}$ & HFIP & 25 & 22 & 0.5 & 15 \\
PBS/PBTDG & HFIP & 25 & 19 & 0.5 & 15 \\
P(BS ${ }_{10}$ BTDG $\left._{10}\right)$ & HFIP & 25 & 23 & 0.5 & 15 \\
\hline
\end{tabular}

a) $D=$ needle-to-collector distance; b) " $m$ " indicates micrometric fibers; c) " $n$ " indicates nanometric fibers.

Table S2. Peak molecular weight as a function of incubation time for PBS/PBDG andPBS/PBTDG.

\begin{tabular}{|c|c|c|c|c|}
\hline & \multicolumn{2}{|c|}{ PBS/PBDG } & \multicolumn{2}{|c|}{ PBS/PBTDG } \\
\hline Incubation time (days) & $M_{p}, 1$ & $M_{p}, 2$ & $M_{p}, 1$ & $M_{p}, 2$ \\
\hline 0 & 40600 & l & 51400 & l \\
\hline 27 & 24700 & 58800 & 37900 & I \\
\hline 47 & 18500 & 58600 & 32000 & 59300 \\
\hline 77 & 14200 & 58600 & 26100 & 59400 \\
\hline 92 & 12000 & 58100 & 18800 & 59000 \\
\hline 109 & 10700 & 56600 & 12500 & 57500 \\
\hline 145 & 8200 & 51100 & 9700 & 52100 \\
\hline 162 & 5400 & 50900 & 8700 & 51000 \\
\hline
\end{tabular}

Table S3. Sequences of primers used in real time RT-PCR.

\begin{tabular}{lll}
\hline Gene & Forward primer $5^{\prime}$ to $3^{\prime}$ & Reverse primer $5^{\prime}$ to $3^{\prime}$ \\
\hline B2M & ACAAAGTCACATGGTTCACA & GACTTGTCTTTCAGCAAGGA \\
ALP & ACAAGCACTCCCACTTCATC & TTCAGCTCGTACTGCATGTC \\
RUNX2 & TGGTTACTGTCATGGCGGGTA & TCTCAGATCGTTGAACCTTGCTA \\
COL1A1 & GAGGGCCAAGACGAAGACATC & CAGATCACGTCATCGCACAAC \\
BSP & CCCCACCTTTTGGGAAAACCA & TCCCCGTTCTCACTTTCATAGAT \\
COL2A1 & CGTCCAGATGACCTTCCTACG & TGAGCAGGGCCTTCTTGAG \\
SOX9 & TGGGCAAGCTCTGGAGACTTC & ATCCGGGTGGTCCTTCTTGTG \\
ACAN & AGGCAGCGTGATCCTTACC & GGCCTCTCCAGTCTCATTCTC \\
ALCAM & ACGATGAGGCAGACGAGATAAGT & CAGCAAGGAGGAGACCAACAAC \\
\hline
\end{tabular}




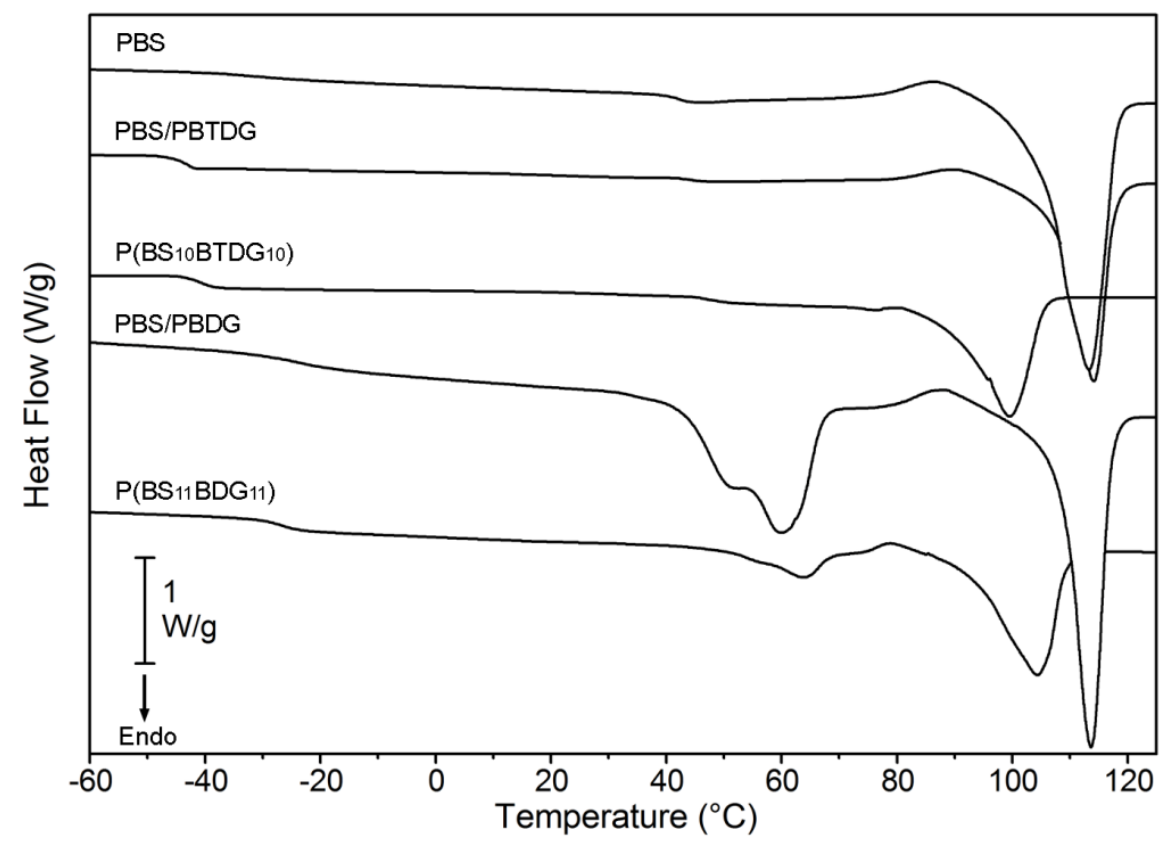

Figure S1. Calorimetric curves of the polymeric scaffolds under investigation.
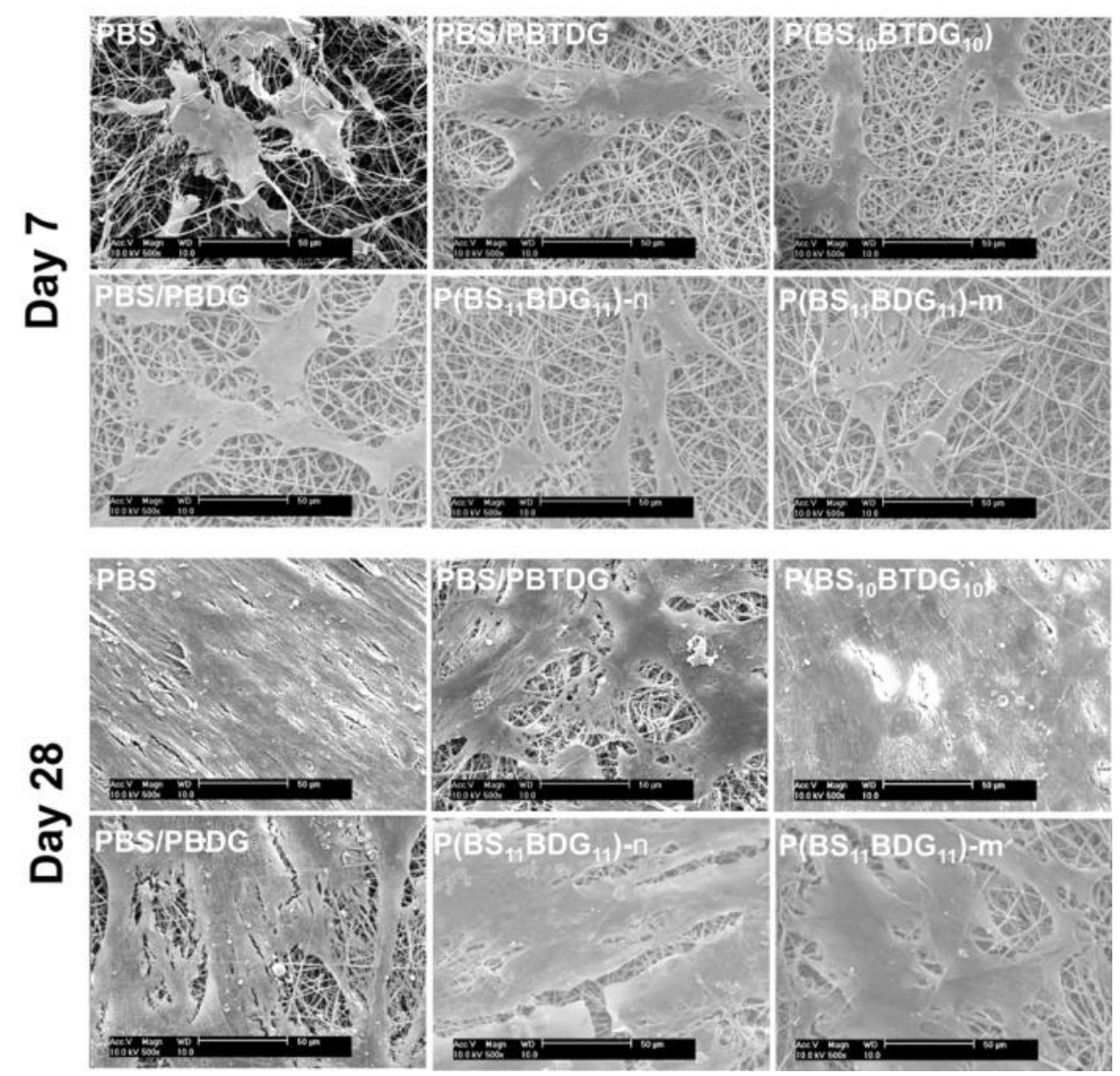

Figure S2. SEM images of hMSCs cultured on scaffolds in BM at day 7 and day 28. 

and Thio-ether Linkages for Skeletal Differentiation of Human Mesenchymal Stromal Cells
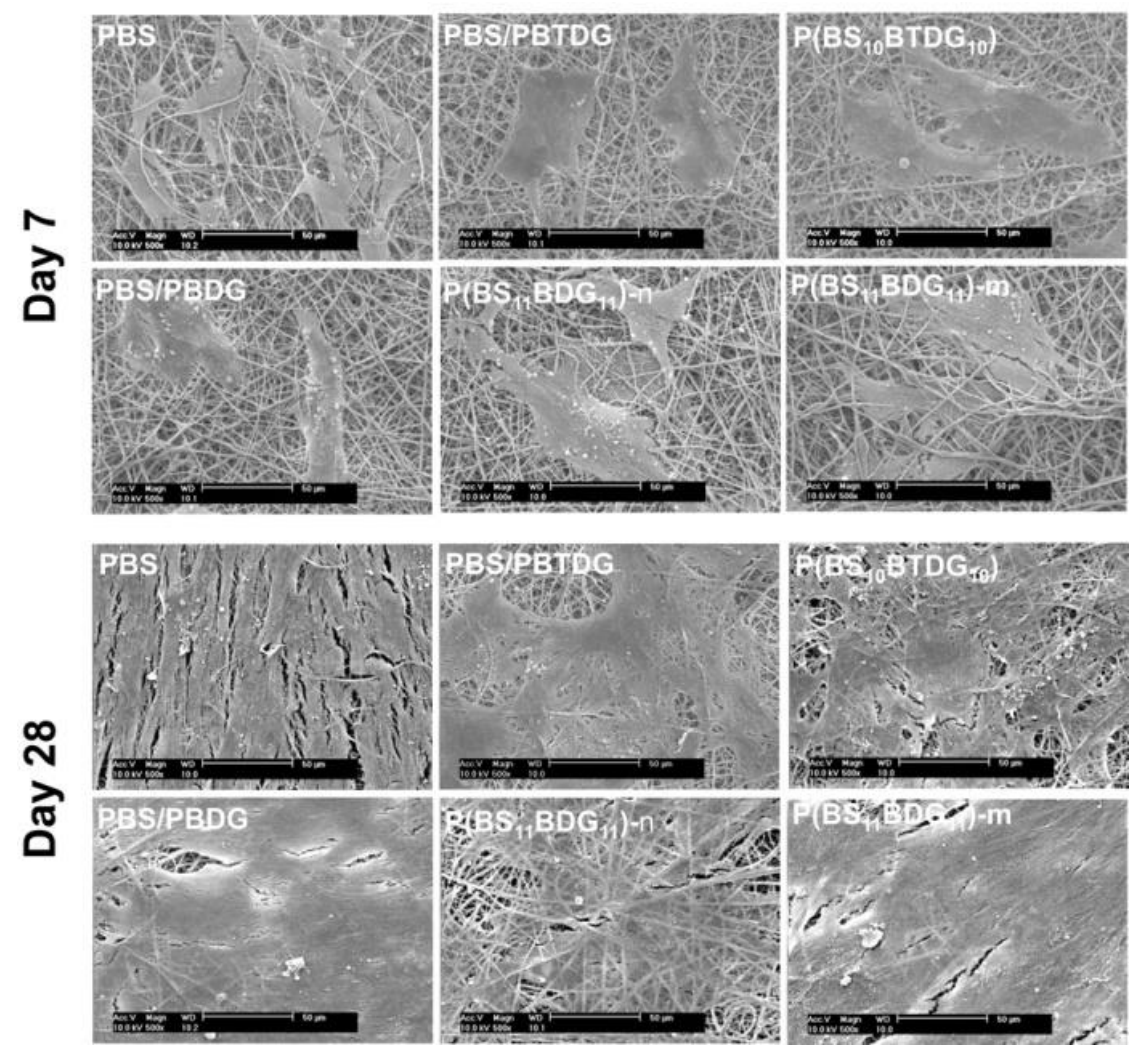

Figure S3. SEM images of hMSCs cultured on scaffolds in OM at day 7 and day 28.

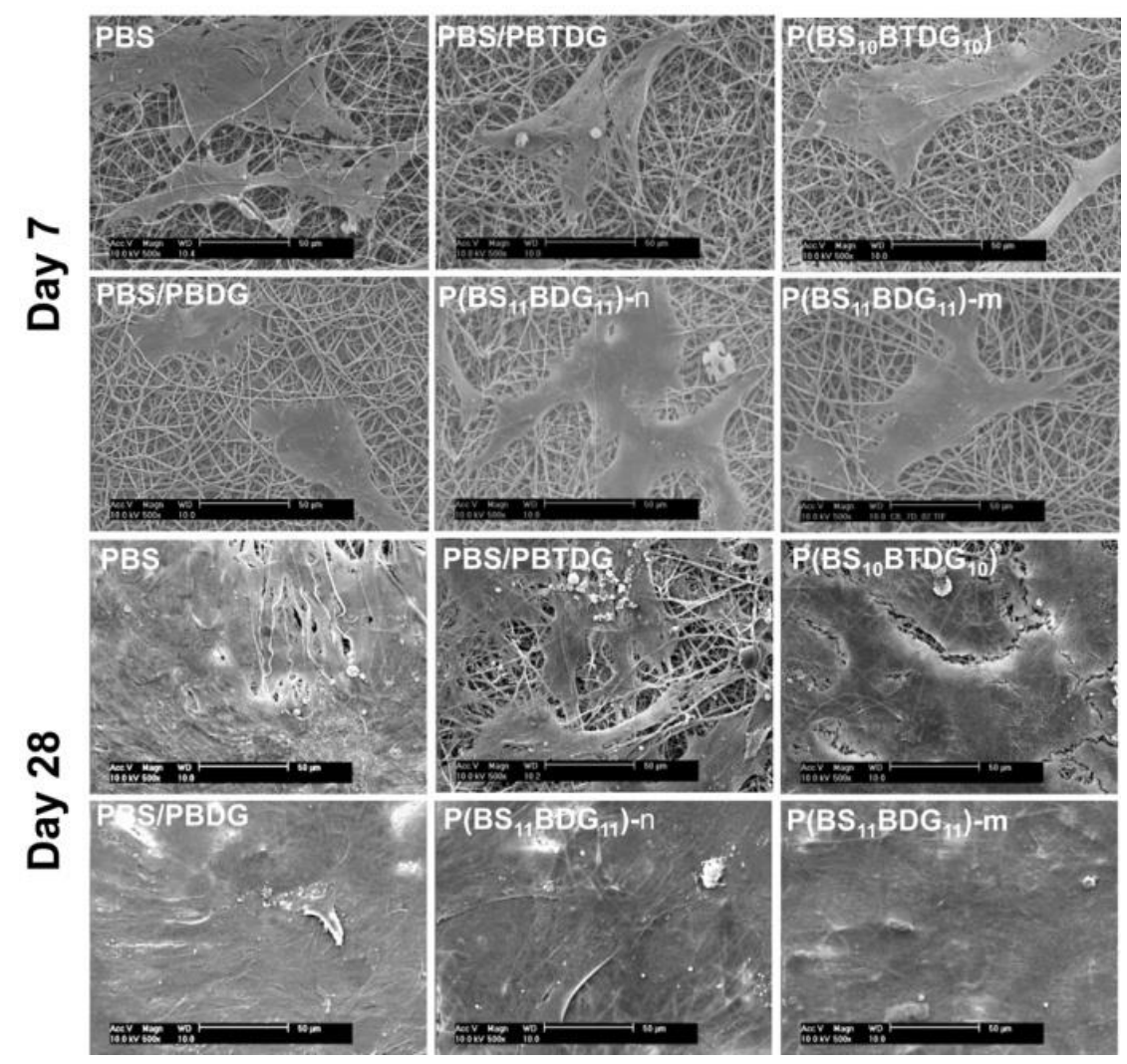

Figure. S4. SEM images of hMSCs cultured on scaffolds in CM at day 7 and day 28. 
A

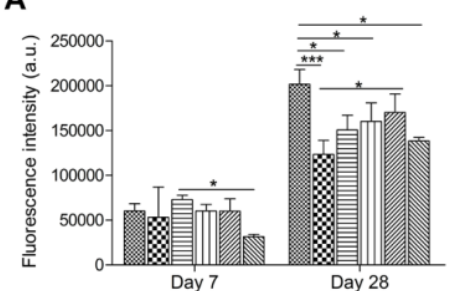

B

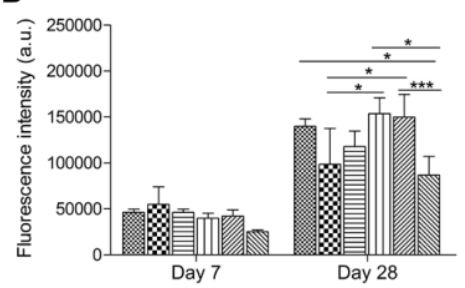

C

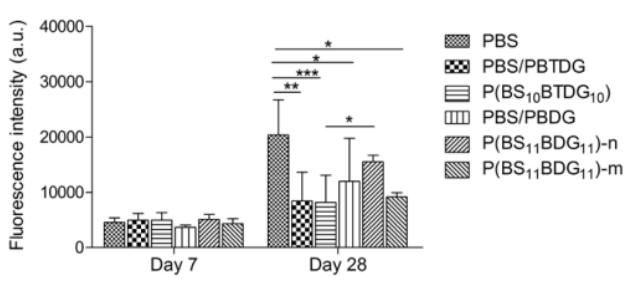

Figure. S5. Metabolic activity of hMSCs cultured on scaffolds in $B M(A), O M(B)$ and $C M(C)$ at day 7 and day 28. ${ }^{*} p<0.05,{ }^{* *} p<0.01,{ }^{* * *} p<0.001$.

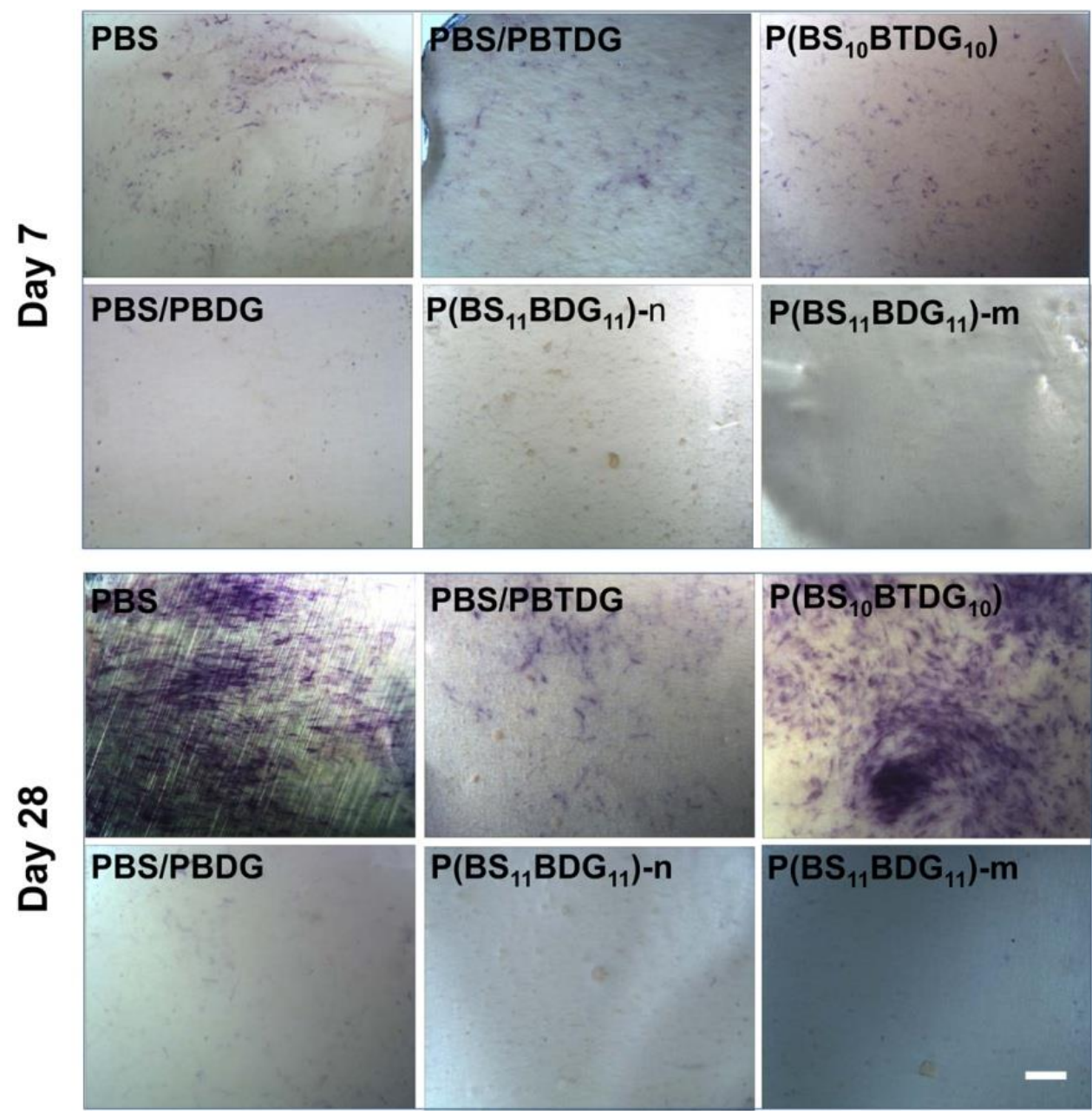

Figure. S6. The staining of alkaline phosphatase enzyme activity for hMSCs on scaffolds in BM at day 7 and day 28. Scale bar $=1 \mathrm{~mm}$. 

and Thio-ether Linkages for Skeletal Differentiation of Human Mesenchymal Stromal Cells
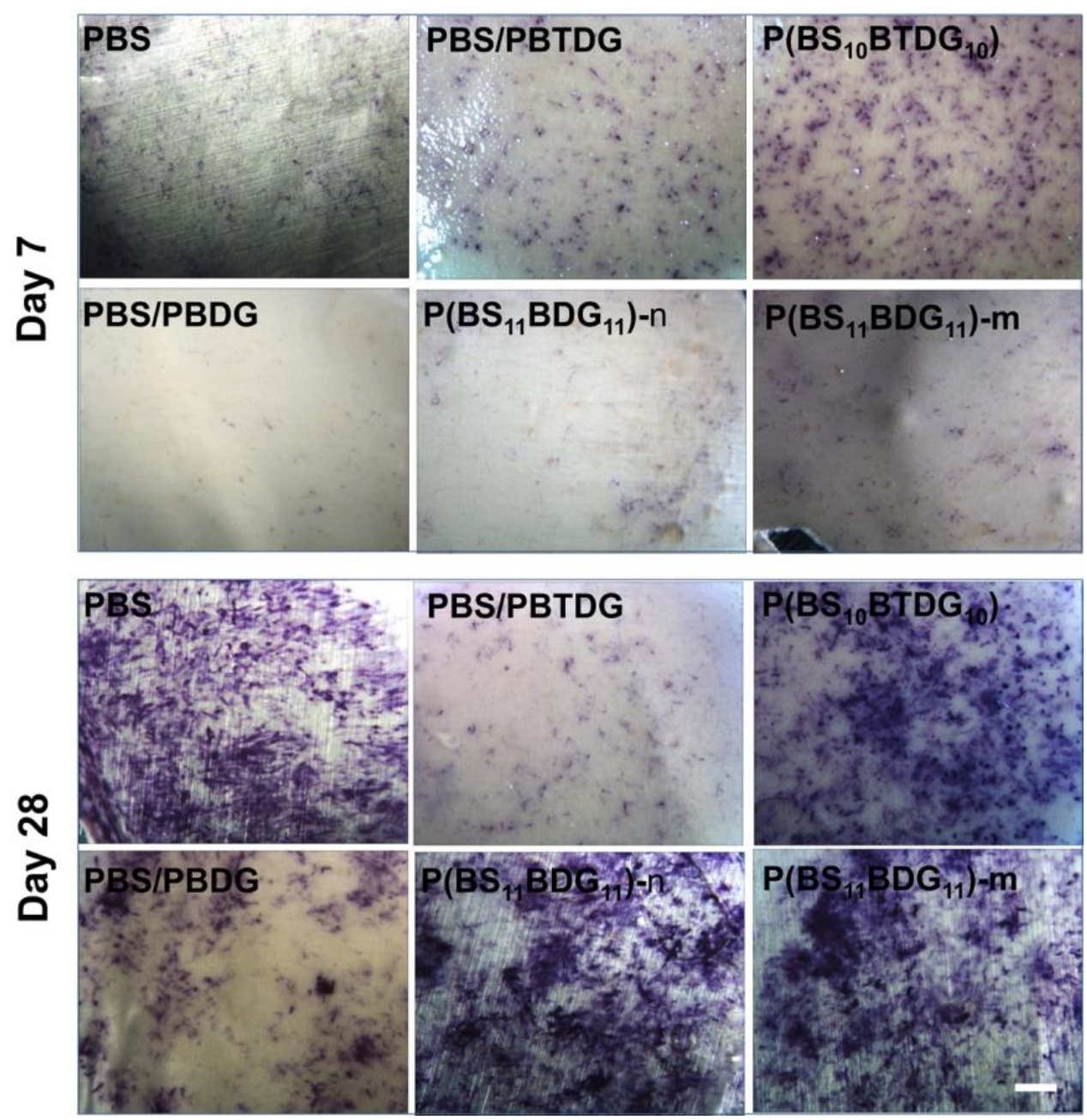

Figure. S7. The staining of alkaline phosphatase enzyme activity for hMSCs on scaffolds in OM at day 7 and day 28. Scale bar $=1 \mathrm{~mm}$ 


\section{Chapter 3}

\section{Flexible Yttrium-Stabilized Zirconia Nanofibers Offer Bioactive Cues for Osteogenic Differentiation of Human Mesenchymal Stromal Cells}

Gerard Cadafalch Gazquez ${ }^{1+]}$, Honglin Chen ${ }^{2[+]}$, Sjoerd A. Veldhuis ${ }^{1}$, Alim Solmaz', Carlos Mota ${ }^{2}$, Bernard A. Boukamp ${ }^{1}$, Clemens A. van Blitterswijk², Johan E. ten Elshof ${ }^{1}$, and Lorenzo Moroni ${ }^{2}$

${ }^{1}$ Inorganic Materials Science Group, MESA+ Institute for Nanotechnology, University of Twente, $7500 \mathrm{AE}$ Enschede, The Netherlands

${ }^{2}$ Department of Complex Tissue Regeneration, MERLN Institute for Technology Inspired Regenerative Medicine, 6200 MD Maastricht, The Netherlands

${ }^{[+]}$These authors contributed equally to this work. 


\section{Abstract}

Currently, the main drawback of ceramic scaffolds used in hard tissue regeneration is their low mechanical strength. Stabilized zirconia, especially the tetragonal $3 \%$ yttriumstabilized zirconia (YSZ) phase, has been considered as a bioinert ceramic material with high mechanical strength. In the present work, novel flexible nanofibrous YSZ scaffolds were prepared by electrospinning. The obtained scaffolds showed remarkable flexibility at the macroscopic scale, while retaining their stiffness at the microscopic scale. The surface nanoroughness of the scaffolds could be tailored by varying the heat treatment methods. Our results demonstrate the osteogenic differentiation and mineralization of seeded human mesenchymal stromal cells (hMSCs) were supported by the nanofibrous YSZ scaffolds, in contrast to the well-known bioinert behavior of bulk YSZ. These findings highlight that flexible ceramic scaffolds are an appealing alternative to the current brittle ceramics for bone regeneration applications.

\section{Keywords:}

Flexible ceramic scaffolds, electrospinning ceramic, surface roughness, human mesenchymal stromal cells, osteogenic differentiation 


\subsection{Introduction}

Bioceramics have been widely used for several decades to repair bone damage[1-3], since they are promising materials due to their similarity to bone in terms of chemical properties[4-7]. Within the area of bioceramics, a clear distinction is made between bioinert and bioactive materials. Bioinert ceramics can be defined as those that do not bond with the surrounding tissues, nor induce a cell response. Examples of bioinert materials are alumina and yttria-stabilized zirconia (YSZ) [2, 3]. Conversely, bioactive ceramics are materials that can actively induce a specific cell response (e.g. cell differentiation) and, in some cases, are resorbable. These materials include hydroxyapatite (HA), $\beta$-calcium phosphates and bioactive glasses [1-3]. One of the major limitations of ceramic scaffolds for hard tissue regeneration is that they suffer from low mechanical strength, which may facilitate fragile failure when implanted [4, 6, 8]. Among the aforementioned bioceramic materials, zirconia has the highest mechanical strength, especially the $3 \%$ yttrium-stabilized tetragonal zirconia (YSZ) phase $[9,10]$.

The scaffold's microstructure also plays a crucial role. Microporosity provides a large surface area for cell adhesion, thus improving the biological response [11]. However, the scaffolds' mechanical properties are negatively affected [4, 7]. Porosity reduces the mechanical strength of materials [7]. Tailor-made design of the nanoarchitecture of porous biomaterials may help to overcome these issues [9, 12-14]. A recent study demonstrated a nanoarchitectured aluminosilicate ceramic with improved ductility and flexible recovery after compressive stresses [12]. Nanofiber mats of titania, silica and zirconia with enhanced flexibility have also been reported [13-20].

To fabricate scaffolds with fiber dimensions on the nano- to microscale mimicking the features of the natural extracellular matrix (ECM), electrospinning has been used broadly in the field of scaffold tissue engineering [21-23]. Cells do not only respond to chemical and physical stimuli from their neighboring cells, but also from the surrounding fluid and ECMs [24]. The characterization and control of surface properties, such as surface roughness, can therefore be used to optimize cell attachment, proliferation and differentiation.

In this article, we propose the fabrication of a flexible YSZ nanofiber material for bone tissue engineering. Recently, YSZ-based fibers have been fabricated by electrospinning for various applications, such as fuel cells [25] and filtration [18], but their application in the biological and biomedical field has not been explored to our knowledge. Herein, we introduce novel YSZ ceramic nanofibers in the field of regenerative medicine. The YSZ 
fibers typically have lengths of the order of meters, instead of just a few millimetres as reported earlier [25], and a high gravimetric density. The sintering temperature that we employed to densify the ceramic nanofibers was much lower than in earlier studies [25], so that sintering between fibers was avoided, which contributed further to their flexibility and retention of mechanical properties upon cyclic deformation. Indeed, these YSZ ceramic nanofibers have remarkable flexibility compared to the traditional bioactive ceramics and their surface nanoroughnesses can be tailored for cellular response. Interestingly, in contrast to the well-known bioinert behavior of bulk YSZ, nanofiber meshes support in vitro osteogenic differentiation and mineralization of human mesenchymal stromal cells (hMSCs). This is the first study showing that flexible YSZ ceramic fibers with tailored surface nanoroughness can conduct osteogenic differentiation of hMSCs. The flexibility of the scaffold helps to overcome the problem of the fragile fracture that is characteristic for traditional bioceramic scaffolds. Therefore, it provides a complement to the currently available library of bioactive ceramic materials for bone tissue engineering.

\subsection{Experimental procedures}

\subsubsection{Electrospinning}

Zirconium n-propoxide (Alfa Aesar, Lancashire, U.K.) and yttrium acetate hexahydrate (Alfa Aesar, Lancashire, U.K.) were dissolved in n-propanol (Sigma Aldrich) in a 97 to 3 $\%$ molar ratio. Then, polyvinyl pyrrolidone (PVP) (M.W. $1.3 \mathrm{~kg} / \mathrm{mol}$, Sigma Aldrich, St. Louis, U.S.A.) was added to the solution and left stirring overnight to ensure complete dissolution of the PVP. The solution preparation was performed in a nitrogen atmosphere. Subsequently, the solution was placed into a syringe, which was later connected to Teflon tubing with a $0.8 \mathrm{~mm}$ needle and place in the needle holder of the electrospinning setup. The flow rate was set at $1 \mathrm{~mL} / \mathrm{h}$, the air gap was $20 \mathrm{~cm}$, the voltage was $15 \mathrm{kV}$ and the temperature and the relative humidity were set to $25{ }^{\circ} \mathrm{C}$ and $30 \%$, respectively.

\subsubsection{Thermal annealing}

The YSZ- PVP fibres were annealed to burn out the organic phase and to obtain a pure YSZ crystalline phase. The samples were annealed either in a conventional oven (CO) (Carbolite, Hope Valley, U.K.) or a microwave oven (MW) (Milestone MicroSynth, Shelton, U.S.A.) at different heating rates. The samples annealed in the $\mathrm{CO}$ were heated at 1 and $5 \stackrel{\circ}{\circ} \mathrm{C} / \mathrm{min}$ to $850{ }^{\circ} \mathrm{C}$. The samples annealed in the MW were either heated at 5 
${ }^{\circ} \mathrm{C} /$ min to $850^{\circ} \mathrm{C}$, or by rapid thermal annealing (RTA). RTA consisted on placing the sample directly in a pre-heated $\mathrm{MW}$ at $850{ }^{\circ} \mathrm{C}$, and after annealing the samples were cooled to room temperature without a temperature program.

\subsubsection{Fiber morphology and diameter}

The fiber morphology was studied by a Zeiss Merlin (Oberkochen, Germany) scanning electron microscope at high magnification. The fiber diameter was determined from low magnification SEM pictures. The imaging was done at $1.4 \mathrm{keV}$. Fifty measurements were performed per micrograph and per sample, respectively.

\subsubsection{Phase identification and crystallite size}

The crystallinity and phase purity of the samples were studied by $\mathrm{X}$-ray diffraction in the $2 \theta$ range between $10^{\circ}$ and $100^{\circ}$ using a PANAlytical X'Pert (Almelo, The Netherlands), with a step size of $0.015^{\circ}$ and $8 \mathrm{~s}$ per step. The crystallite size was estimated using the Scherrer equation from the full width at half maximum of the (111) peak. The Scherrer equation is presented in equation 1.

$\tau=\frac{K \lambda}{\beta \cos \theta}$

Where $\mathrm{T}$ is the crystallite size, $K$ is the dimensionless shape factor, $\lambda$ is the $X$-ray wavelength, $\beta$ the full width at half maximum of the peak and, $\theta$ is the Bragg's diffraction angle.

\subsubsection{Macroscopic mechanical properties}

The mechanical properties of the ceramic nanofiber mats $(n=3)$ were measured by means of microindentation. A Piuma microindenter (Optics 11, Amsterdam, The Netherlands) equipped with a tip with a radius of $37.5 \mu \mathrm{m}$ and a spring constant of $199 \mathrm{~N} / \mathrm{m}$ was utilized. 25 points were scanned throughout $2500 \mu \mathrm{m} 2$ for each sample.

\subsubsection{Microscopic mechanical properties and surface morphology}

The microscopic mechanical properties were measured by a 3-point bending test on a single nanofiber, as previously reported elsewhere [26, 27]. A polycarbonate substrate with $90 \mu \mathrm{m}$ wide channels was used as a substrate to suspend the fibers. An atomic force microscope (Bruker Icon, Massachusetts, U.S.A.) equipped with a ScanAsyst-AIR (Bruker, Massachusetts, U.S.A.) tip was used to measure the force/displacement curves of the fiber. The tip had a spring constant of $0.4 \mathrm{~N} / \mathrm{m}$ and a resonance frequency of 70 
$\mathrm{kHz}$. The apparent Young's modulus was calculated with the Euler/Bernulli beam theory (Equation 2) using the average fiber diameter obtained from SEM.

$E^{*}=\left(\frac{F}{d}\right) \frac{L^{3}}{12 \pi r D^{4}}$

Here $E^{*}$ is the apparent Young's modulus, $F / d$ is the slope of the force displacement curve, $L$ is the gap distance and $D$ is the fiber diameter. The fiber surfaces were also scanned using the same tip in tapping mode to obtain surface morphology data. The roughness values $(\mathrm{Rq})$ were obtained by analyzing the AFM topography data using the Gwyddion software package (version 2.31).

\subsubsection{Fatigue test}

A three-point bending fatigue test was performed on the ceramic nanofiber mats. This version of the 3-point bending test was especially designed for the experiment, and consisted of (1) a plate with a well-defined gap, on which the sample was suspended on, and (2) a knife, which applied a force to deflect the sample. The gap was $2 \mathrm{~mm}$ wide and the knife was $0.5 \mathrm{~mm}$ thick. The width of the sample was kept constant at $10 \mathrm{~mm}$. The plate and knife were set on a rheometer (Anton Paar Physica MCR 501, Graz, Austria), which measured the force displacement curves at a speed of $50 \mu \mathrm{m} / \mathrm{s}$.

The sample's deflection was calculated assuming a trigonometric system between the sample, knife, and gap. Thus, if the displacement of the knife is known, the deflection angle may be calculated using half of the gap distance. This is exemplified in Supplementary Figure 8.

\subsubsection{Cell Culture}

Pre-selected hMSCs (donor No. 8001L) were isolated from human bone marrow by the Institute of Regenerative Medicine (Texas A\&M University, Temple, Texas) which provide standardized preparations of MSCs for research use under the auspices of an NIH/NCRR grant (P40 RR 17447-06) [28, 29]. Briefly, mononuclear cells were separated using density centrifugation and plated to obtain adherent hMSCs. The cells were harvested around a confluence of 60-80\%, after which they were considered as passage zero (P0). The P0 cells were further expanded and harvested to obtain passage 1 (P1). To obtain sufficient cells for scaffold-based experiments, hMSCs (P1) were expanded in T-300 flasks at a density of $3 \times 10^{5}$ cells $/ \mathrm{cm}^{2}$ and cultured in BM, consisting of $\alpha-M E M$ (Gibco), $10 \%$ fetal bovine serum (Lonza), $2 \mathrm{mM} \mathrm{L-glutamine} \mathrm{(Gibco),} 0.2 \mathrm{mM}$ ascorbic acid (Sigma), $100 \mathrm{U} / \mathrm{mL}$ penicillin and $100 \mathrm{mg} / \mathrm{mL}$ streptomycin (Gibco). The cells were 
harvested at approximately $80-90 \%$ confluence for further sub-cultures.

\subsubsection{Cell seeding on scaffolds}

The ceramic scaffolds were cleaned using oxygen plasma cleaning (Harrick Plasma PDC-002) for $10 \mathrm{~min}$ at high power and subsequently punched out into discs to fit inside a 24-well plate. Rubber O-rings (Eriks B.V., The Netherlands) were inserted into the well to hold the substrate on the bottom. The ceramic scaffolds were sterilized in $70 \%(\mathrm{v} / \mathrm{v})$ ethanol for 15 min each time for 3 times. After sterilization, the scaffolds were rinsed with PBS for 5 min each time for 3 times and immersed in $\mathrm{BM}$ at $37^{\circ} \mathrm{C}$ overnight before cell seeding. Ceramic scaffolds were seeded at a density of $2 \times 10^{4} \mathrm{cells} / \mathrm{cm}^{2}$ and cultured in an incubator with $5 \% \mathrm{CO}_{2}$ humid atmosphere at $37^{\circ} \mathrm{C}$ for up to 14 days in $\mathrm{OM}$, and in both $\mathrm{BM}$ and $\mathrm{MM}$ for 28 days. BM is considered a normal growth medium, which cannot induce osteogenic differentiation nor form a mineralized matrix[30, 31]. OM consisted of BM supplemented with $10^{-8} \mathrm{M}$ dexamethasone to induce osteogenic differentiation of hMSCs and the MM consisted of OM supplemented with $\beta$-glycerophosphate to support the osteogenic differentiation[30, 31], and promote the formation of a mineralized matrix. The culture medium was refreshed every 2 days.

\subsubsection{Live and dead assay}

To determine the cell viability on scaffolds, a LIVE/DEAD $^{\circledR}$ assay (Life Technologies) was performed. After washing the scaffolds twice with PBS, they were incubated for $30 \mathrm{~min}$ with a mixture of PBS solution containing ethidium homodimer (red fluorescent) / calceinAM (green fluorescent) at room temperature following the manufacturer's protocol. Scaffolds were washed three times with PBS before imaging with a fluorescence microscope (Nikon, Eclipse E600 and Nikon DS-Filc camera).

\subsubsection{SEM analysis}

After 3 days of culture, the medium was removed, and the scaffolds were washed two times with PBS and fixed in a $10 \%$ formalin solution for $2 \mathrm{~h}$. After rinsing with PBS, the scaffolds were dehydrated with ethanol series $(50 \%, 70 \%, 80 \%, 90 \%, 96 \%$, and $100 \%)$. The scaffolds were further processed by critical point drying (Balzers CPD-030). The dried samples were sputtered with gold (Cressington) and imaged with a XL-30 ESEMFEG SEM (FEI, Eindhoven, Netherlands). 


\subsubsection{Fluorescence microscopy}

On day 3, samples for phalloidin staining were fixed with $4 \%(\mathrm{v} / \mathrm{v})$ paraformaldehyde (Sigma-Aldrich) in PBS, left overnight at $4{ }^{\circ} \mathrm{C}$ and subsequently washed 3 times with PBS. Afterwards, the cell membrane was permeabilized with $0.5 \%$ TritonX-100 solution for 10 min at $4{ }^{\circ} \mathrm{C}$ and washed with PBS again. A $1 \%$ BSA in PBS solution was added for $2 \mathrm{~h}$ to block non-specific binding. After withdrawing the BSA solution, phalloidin (Alexa Fluor 488, Invitrogen; Excitation/Emission:495/518 nm) with a dilution (1:40) in 1\% BSA/PBS was applied for 25 min at room temperature (RT) in the dark and rinsed 3 times with PBS. For nuclei staining, DAPI (1:100) in PBS was applied for 15 min at RT in the dark. The samples were observed under a fluorescence microscope (Eclipse E600, Nikon) equipped with proper filter. The average surface area for single cells was determined by the software CellProfiler 2.1.1(Broad Institute, Cambridge, USA). The surface area in each of at least 10 cells within an image for a total of six images per sample was quantified. All these images were from the same experiment.

\subsubsection{Cell metabolic activity}

The cell metabolic activity on day 7 and day 14 was assessed using a PrestoBlue ${ }^{\mathrm{TM}}$ Cell Viability Reagent (Life technologies), according to the manufacturer's protocol. Briefly, a cultured medium was replaced with a medium containing $10 \%(\mathrm{v} / \mathrm{v})$ PrestoBlue reagent and cells were incubated for $1 \mathrm{~h}$ at $37{ }^{\circ} \mathrm{C}$. Fluorescence was measured at $590 \mathrm{~nm}$ on a Perkin Elmer Victor3 1420 multiple plate reader. Three separate experiments $(n=3)$ and two replicates each were performed.

\subsubsection{ALP activity}

The relative ALP activity was measured by chemoluminescence with a CDP-star ${ }^{\circledR}$ kit (Roche Life Science), according to the manufacturer's instruction. Briefly, cell-scaffold constructs were washed 3 times with PBS and stored at $-80^{\circ} \mathrm{C}$ for at least $24 \mathrm{~h}$. After thawing, the constructs were treated with $250 \mu \mathrm{L}$ cell lysis buffer at a pH of 7.8 (composed of $0.1 \mathrm{M} \mathrm{KH}_{2} \mathrm{OPO}_{4}, 0.1 \mathrm{M} \mathrm{K}_{2} \mathrm{HPO}_{4}$ and $0.1 \%$ Triton $\mathrm{X}-100$ ) for $1 \mathrm{~h}$ at RT. Cell lysates $(50 \mu \mathrm{L})$ were transferred to Eppendorf tubes and stored at $-20{ }^{\circ} \mathrm{C}$ for ALP assay. The remainder of the remaining cell lysates $(200 \mu \mathrm{L})$ was used for DNA assay. For ALP analysis, $10 \mu \mathrm{L}$ of cell lysates were transferred in duplicate to a well of a 96-well plate. Subsequently, $40 \mu \mathrm{L}$ of CDP-star were added and plates were incubated for $15 \mathrm{~min}$ at room temperature. The plates were measured on a Multilabel reader (Victor3 1420, 
Perkin Elmer, USA). Three separate experiments $(n=3)$ and two replicates each were performed. The results of ALP activity were presented after normalization to DNA quantity per scaffold.

\subsubsection{Quantification of DNA amount}

Cell lysates remainder, $(200 \mu \mathrm{L})$ as mentioned above, were further digested for $16 \mathrm{~h}$ at $56^{\circ} \mathrm{C}$ with $200 \mu \mathrm{L}$ Tris/EDTA buffer containing $1 \mathrm{mg} / \mathrm{mL}$ proteinase $\mathrm{K}$ (Sigma Aldrich), 185 $\mu \mathrm{g} / \mathrm{mL}$ iodoacetamine (Sigma Aldrich) and $10 \mu \mathrm{g} / \mathrm{mL}$ Pepstatin A (Sigma Aldrich). The quantification of the total amount of DNA was performed using the CyQuant ${ }^{\circledR}$ DNA assay (Molecular Probes, Eugene, USA) according to the manufacturer's manual. Briefly, a 40 $\mu \mathrm{L}$ aliquot of lysate was pipetted in duplicate into a black 96-well plate, followed by addition of $40 \mu \mathrm{L}$ RNase (1000x) in NaCl-EDTA buffer composed of component B (20x) and incubated for $1 \mathrm{~h}$ at RT. Subsequently, $80 \mu \mathrm{L}$ of $\mathrm{Gr}$-dye solution was added and incubated for $15 \mathrm{~min}$. The measurements were performed on a spectrophotometer reader (Victor3 1420, Perkin Elmer, USA) at an excitation wavelength of $480 \mathrm{~nm}$ and an emission wavelength of $520 \mathrm{~nm}$. Three separate experiments $(n=3)$ and two replicates each were performed.

\subsubsection{Gene expression analysis}

Total RNA was isolated from samples at day 7 and 14 using a combination of TRIzol with a NucleoSpinRNAll isolation kit (Bioke). Briefly, the scaffolds were washed three times with PBS and $1 \mathrm{~mL}$ of TRIzol was added for each sample. Then, $0.2 \mathrm{~mL}$ of chloroform was added and the samples were centrifuged for $15 \mathrm{~min}$ at 12,000 RCF (relative centrifugal force). The aqueous phase containing RNA was transferred to a new Eppendorf $\AA$ tube, mixed with an equal volume of $75 \%(\mathrm{v} / \mathrm{v})$ ethanol and loaded onto an RNA binding column of the isolation kit. After a series of purification procedures, the RNA was re-suspended in RNASE-free water. RNA concentration and purity were measured by using an ND 1000 spectrophotometer (Nanodrop Technologies, USA). Finally, cDNA was synthesized using iScript (Bio-Rad, Veenendaal, The Netherlands) according to the manufacturer's protocol. Quantitative PCR was performed using SYBR-green supermix (Bio-Rad). Four separate experiments $(n=4)$ were performed. Cycling parameters for quantitative PCR were as follows: initial denaturation for $10 \mathrm{~min}$ at $95^{\circ} \mathrm{C}$, followed by 40 cycles of $15 \mathrm{~s}$ at $95^{\circ} \mathrm{C}$ and $1 \mathrm{~min}$ at $60^{\circ} \mathrm{C}$. The sequences of the analyzed primers are 
shown in Table 1. Cycle threshold (CT) values were normalized to the housekeeping gene $\mathrm{B}_{2} \mathrm{M}$, and the results of gene expression were calculated as $2^{-\Delta C T}$.

\subsubsection{Alizarin red $S$ staining and quantification}

After 28 days of culture, samples were washed with PBS, fixed with $10 \%$ formalin for $1 \mathrm{~h}$, and then subsequently with PBS and twice with distilled water. A freshly filtered $2 \%$ aqueous alizarin red $\mathrm{S}$ solution ( $\mathrm{pH}$ 4.2) was applied to scaffolds for 2 min, after which the excess solution was washed off with distilled water. The quantification of orange-red coloration of alizarin red $S$ was carried out as in previous studies[32, 33]. Briefly, constructs were transferred to a microcentrifuge tube before removing excess water. $A$ $10 \%$ acetic acid solution was added and samples were shaken for 30 min at RT. After vortexing for $30 \mathrm{~s}$, the solution was centrifuged for $15 \mathrm{~min}$ at 20,000 RCF and the supernatant phase was removed to a new $1.5 \mathrm{~mL}$ microcentrifuge tube. A $10 \%$ ammonium hydroxide solution was added drop-wise to neutralize the acid. Aliquots of the supernatant phase were measured in triplicate at $405 \mathrm{~nm}$ in a 96-well plate using opaquewalled, transparent-bottomed plates (Fisher Lifesciences). Three separate experiments (n $=3$ ) and two replicates each were performed. Scaffolds without seeding cells were used as control.

Table 1. Sequences of primers used in PCR.

\begin{tabular}{lll}
\hline Gene & Forward primer 5 ' to $3^{\prime}$ & Reverse primer $5^{\prime}$ to $3^{\prime}$ \\
\hline BMP-2 & ACTACCAGAAACGAGTGGGAA & GCATCTGTTCTCGGAAAACCT \\
BSP & CCCCACCTTTTGGGAAAACCA & TCCCCGTTCTCACTTTCATAGAT \\
OPN & CTCCATTGACTCGAACGACTC & CAGGTCTGCGAAACTTCTTAGAT \\
OCN & TGAGAGCCCTCACACTCCTC & CGCCTGGGTCTCTTCACTAC \\
ALP & ACAAGCACTCCCACTTCATC & TTCAGCTCGTACTGCATGTC \\
RUNX2 & TGGTTACTGTCATGGCGGGTA & TCTCAGATCGTTGAACCTTGCTA \\
COL1A1 & GAGGGCCAAGACGAAGACATC & CAGATCACGTCATCGCACAAC \\
B2M & ACAAAGTCACATGGTTCACA & GACTTGTCTTTCAGCAAGGA \\
\hline
\end{tabular}




\subsubsection{Statistics}

All data points are expressed as mean values \pm standard deviation (S.D.). Statistical analysis was carried out using GraphPad Prism 5.01 (GraphPad software, San Diego, USA) for windows. The statistical differences were determined by using two-way analysis of variance (ANOVA), followed by a Tukey's post-hoc test unless otherwise mentioned. A value of $p<0.05$ was considered statistically significant.

\subsection{Results and discussion}

The ceramic nanofibers were fabricated by electrospinning with a mixture of a YSZ precursor and polyvinylpyrrolidone (PVP). Subsequently, the resulting fiber mats were annealed by means of conventional or microwave-assisted heating in static air at $850{ }^{\circ} \mathrm{C}$ for $2 \mathrm{~h}$ using different heating rates (i.e. to burn out the organic phases and for crystalline phase formation). The obtained nanofibers had a diameter of $530 \pm 120 \mathrm{~nm}$ (Figure S1), and the fiber mats had a thickness of approximately $120 \mu \mathrm{m}$. A scanning electron microscopy (SEM) image of the material is shown in Figure 1A. The YSZ nanofiber mats exhibited extraordinary flexibility not often demonstrated for pure ceramic materials. It behaved as a paper-like material (Figure 1B and Video S1). The material could achieve total deflection ( $180^{\circ}$ bending) without mechanical failure. Moreover, the deflection could be cycled at least 1000 times without breaking the material when a 3-point bending test was performed. To the best of our knowledge, the fabrication of ceramic nanofiber mats with such high flexibility and good fatigue behavior has not been reported until now. The flexibility of electrospun silica-based mats has been attributed to the amorphous nature of the material, which is therefore more robust than crystalline materials [14, 34]. However, that hypothesis does not explain the behavior of the crystalline YSZ nanofibers reported here (X-ray diffractograms showing crystalline nature are shown in Figure S2. Park et al hypothesized that the flexibility of crystalline $\mathrm{TiO}_{2}$ was the result of the physical entanglement as single nanofibers are brittle [13]. 


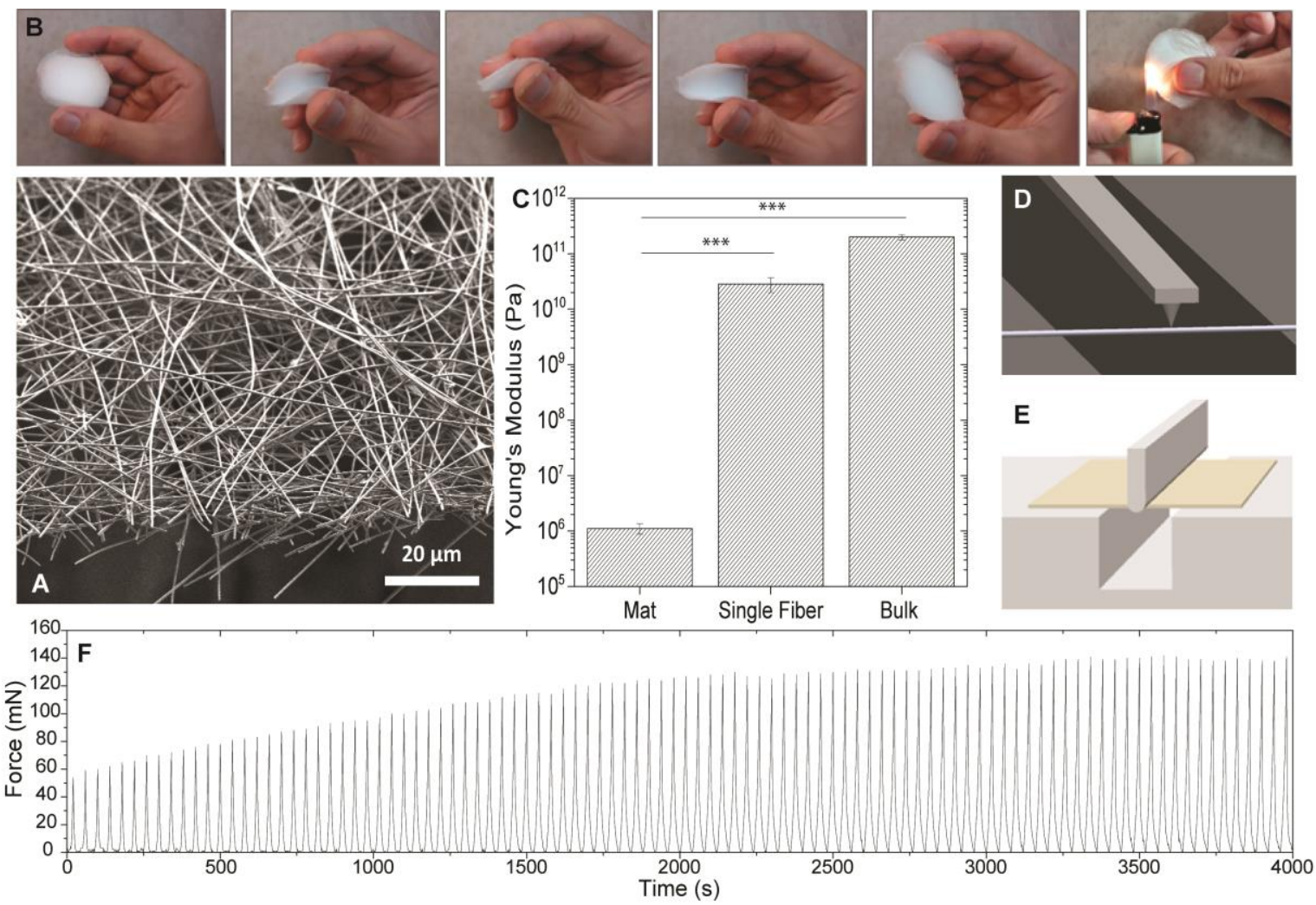

Figure 1. The flexibility of YSZ nanofiber mats. (A) SEM images of a YSZ nanofiber mat, Scale bar is 20 $\mu \mathrm{m}$. (B) Pictures of the YSZ mat showing its flexibility and fire resistance ceramic nature. (C) Mechanical properties of a nanofiber mat by microindentation (at macroscopic scale), a single nanofiber by 3 point bending using AFM (at microscopic scale), and bulk YSZ (taken from literature ${ }^{27-29}$ ). (D) Schematic overview of a 3 point bending test on a single nanofiber using AFM. (E) Schematic illustration of a 3 point bending fatigue test on the nanofiber mat. (F) Representative histogram of 200 cycles of the 3 point bending fatigue test on a YSZ nanofiber mat in which the force was monitored as a function of time. Error bars represent standard deviation of the mean. The statistical significance was determined using a one way ANOVA test, where ${ }^{* * *} p<0.001$.

However, we observed that also single nanofibers were able to withstand a high degree of deflection (Video S2). Probably due to the long length of the nanofibers, the mechanical energy could be dissipated under the elastic deflection. The coherence of fibers mats composed of long fibers is better than that of fiber mats composed of an assembly of fiber fragments. Moreover, the sintering temperature of $850{ }^{\circ} \mathrm{C}$ that we employed is much lower than in earlier reports on YSZ nanofibers where a temperature of $1500{ }^{\circ} \mathrm{C}$ was used [25]. As a result, our YSZ fibers densified but did not sinter together, which contributes to their freedom of movement and thus to their flexibility, and makes them less susceptible to fatigue-like phenomena. The flexibility of the mat was further quantified by microindentation tests. The Young's modulus of the material was $1.11 \pm$ 
0.24 $\mathrm{MPa}$, more than 5 orders of magnitude lower than that of bulk YSZ (Figure 1C), which is in the range of $178-222 \mathrm{GPa}$ according to literature data [9, 35-37]. We also measured the mechanical properties of single nanofibers by a 3-point bending test using atomic force microscope (AFM) (Figure 1D). The measured apparent Young's modulus of a single fiber was $30 \pm 3 \mathrm{GPa}$, and a high deflection angle could be achieved. The apparent Young's modulus of single fibers was several orders of magnitude higher than the macroscopic mat, but still a factor of 7 lower than bulk YSZ (Figure 1C).

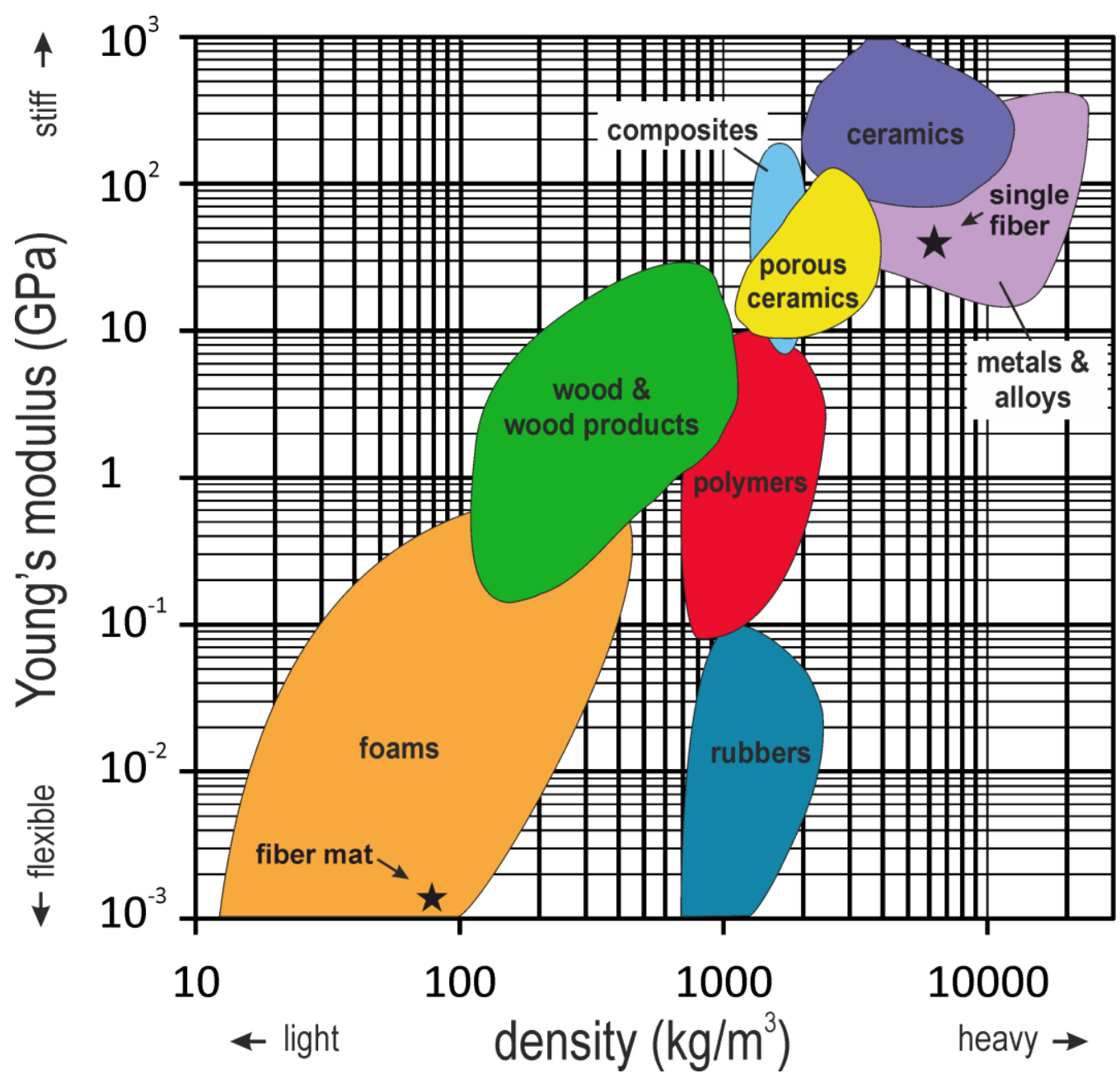

Figure 2. Plot of Young's Modulus vs density. The nanofiber mat and single nanofiber are compared with other traditional materials.

This result is in accordance with other studies on electrospun ceramic nanofibers, which report similar decreases in Young's modulus when measured via a three point bending test using AFM [26, 27]. The diameter and mechanical properties of the nanofibers at the different length scales did not vary significantly with the annealing treatment (Figure S3). 
A special set-up was designed to perform a 3-point bending fatigue test on the mat (Figure 1E). The experiment consisted of 200 cycles of $45^{\circ}$ deflection, while the applied force was monitored (Figure 1F). The sample did not break, and to our surprise we observed a slight increase of the force necessary to deflect the sample. Chen et al. reported slipping of individual nanofibers under tensile stress[18]. It may be possible that such a phenomenon occurs during deflection, gradually leading to a rearrangement of the fibers with an increasing number of cycles, ultimately resulting in a slightly stiffer material.

Moreover, the nanofibers mat offered a very low density, in the range of 0.06-0.09 $\mathrm{g} / \mathrm{cm}^{3}$. Based on SEM observations, i.e. the absence of pores and grain boundaries, each individual fiber can be considered as nearly dense. The Young's modulus of the nanofiber mats and individual fibers were plotted vs. the density as shown in Figure 2. For comparison, the values of other traditional materials were also plotted. It can be seen that the ceramic nanofibers mat behaved like a foam in terms of stiffness and density. This means that it is lighter and more flexible than other polymers, ceramics and porous ceramics. Nevertheless, individual nanofibers offer stiffness values close to ceramics and typical of porous ceramics or metals, 5 orders of magnitude stiffer than the mat.

The surface morphology and grain size of the fibers were modified by annealing the samples under different conditions. The green fiber mats were annealed in a $\mathrm{CO}$ at 1-5 ${ }^{\circ} \mathrm{C} / \mathrm{min}$ heating rate, and in a $\mathrm{MW}$ at $5^{\circ} \mathrm{C} / \mathrm{min}$ or by RTA (i.e. thermal shock). RTA is a method in which the MW is preheated to $850 \stackrel{\circ}{\circ} \mathrm{C}$ prior to placing the sample in the oven. The samples annealed in the $\mathrm{CO}$ showed rougher surface morphologies than the samples annealed in the MW even at the same heating rate, as shown by SEM (Figure $3 \mathrm{~A}$ ) and AFM (Figure 3B) analyses. This was further confirmed by the statistical analysis performed on the roughness data (Figure $3 \mathrm{C}$ ). However, varying the heating rate did not result in significant differences among samples that were annealed by the same heating mechanism. The samples annealed in the $\mathrm{CO}$ at $1{ }^{\circ} \mathrm{C} / \mathrm{min}$ and $5{ }^{\circ} \mathrm{C} / \mathrm{min}$ had roughnesses of $0.8 \pm 0.3 \mathrm{~nm}$ and $0.7 \pm 0.2 \mathrm{~nm}$, respectively. Both samples annealed in the MW had a roughness of $0.4 \pm 0.1 \mathrm{~nm}$. The heating mechanism had a significant influence on surface roughness. Samples annealed at $5{ }^{\circ} \mathrm{C} / \mathrm{min}$ in a $\mathrm{CO}$ had a rougher surface than the samples annealed in a MW at the same heating rate $(0.7 \pm 0.2 \mathrm{~nm}$ vs $0.4 \pm 0.1 \mathrm{~nm})$. These results were consistent with the surface morphologies in SEM images (Figure 3A). 


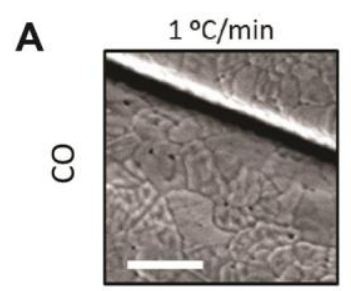

$5^{\circ} \mathrm{C} / \mathrm{min}$

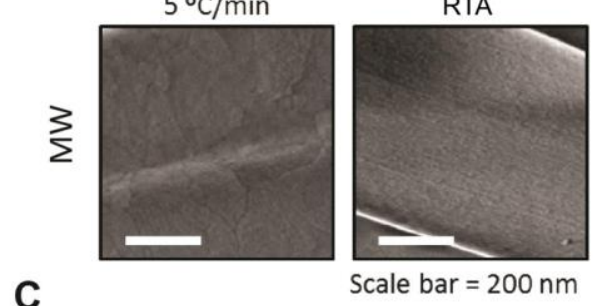

C
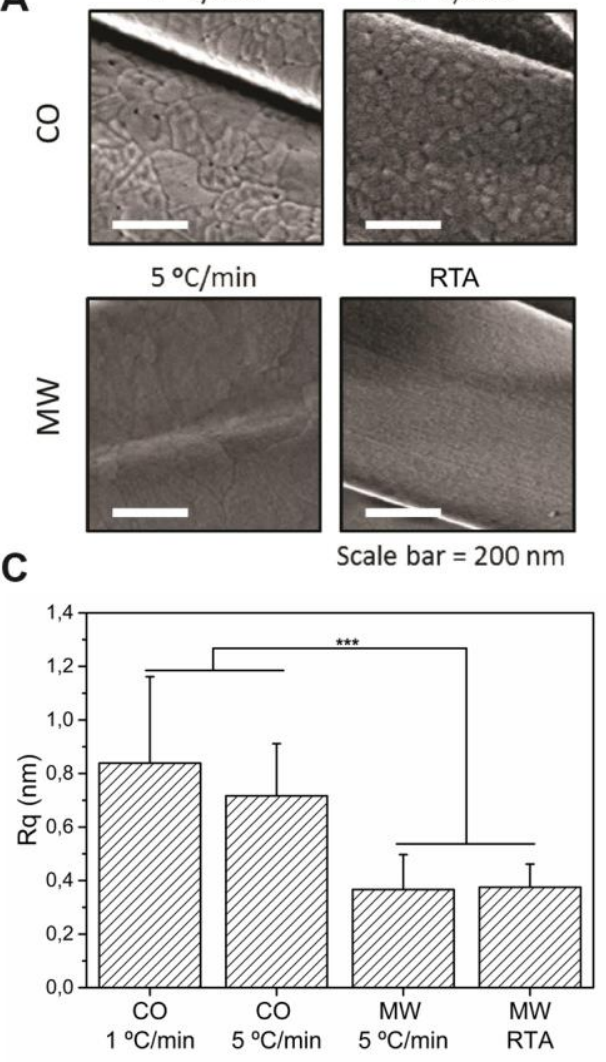

RTA
B

D

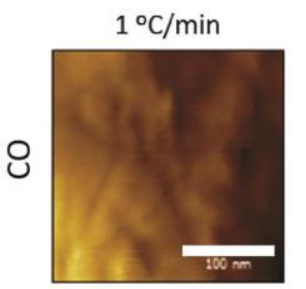

$5^{\circ} \mathrm{C} / \mathrm{min}$

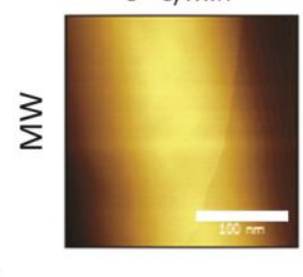

$5^{\circ} \mathrm{C} / \mathrm{min}$

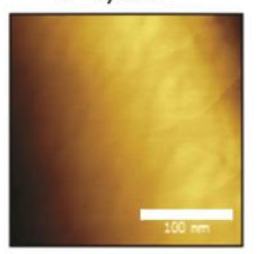

RTA

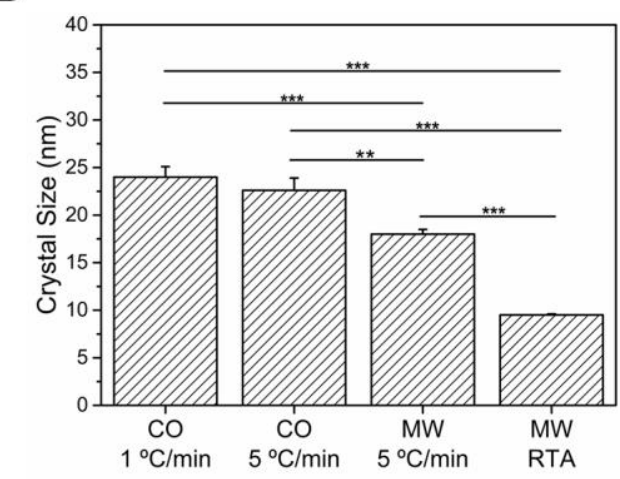

Figure 3. The effect of annealing procedure on the fiber surface morphology. (A) SEM images of YSZ nanofibers, the scale bar is $200 \mathrm{~nm}$. (B) AFM images of YSZ nanofibers, the scale bar is $100 \mathrm{~nm}$. (C) Roughness data extracted from AFM scans. (D) Grain sizes of the fibers calculated from the FWHM of the (111) peak of the XRD patterns. Error bars represent standard deviation of the mean. The statistical significance was determined using a one way ANOVA test, where ${ }^{* *} p<0.01,{ }^{* * *} p<0.001$.

However, it is noted that the roughness with AFM is measured in the out-of-plane direction, whereas the SEM images shows in-plane features of surface morphology. Different grain sizes were observed for each heating rate (Figure 3D) as calculated from the full width at half maximum (FWHM) in the XRD patterns (Figure S2). Samples annealed in the $\mathrm{CO}$ at $1{ }^{\circ} \mathrm{C} / \mathrm{min}$ had the largest grain size, $24 \pm 1 \mathrm{~nm}$. Samples annealed in the $\mathrm{CO}$ and $\mathrm{MW}$ at $5^{\circ} \mathrm{C} / \mathrm{min}$ had intermediate grain sizes of $22 \pm 1 \mathrm{~nm}$ and $18 \pm 1 \mathrm{~nm}$, respectively. The sample annealed in the MW using RTA had a much smaller grain size of $9.5 \pm 0.1 \mathrm{~nm}$. The samples annealed using lower heating rates showed larger grain sizes, irrespective of the heating mechanism. This can be attributed to the longer effective sintering times at high temperature under such conditions. The heating mechanism also affected the grain size. The samples annealed at $5^{\circ} \mathrm{C} / \mathrm{min}$ in a $\mathrm{CO}$ had a larger grain size than the samples annealed in a MW at the same heating rate. YSZ has previously been 
reported to exhibit enhanced sintering behavior under microwave radiation heating[38, 39]. Xie et al. reported smaller and more uniform grain sizes when YSZ was sintered in a MW, which is in accordance with our data (Figure 3D) [39]. The YSZ nanofibers presented here have a different surface nanostructure than any previously reported YSZ topography. They are nearly 1-dimensional objects in a porous matrix, which affects the heat transport and crystallization rates. Nevertheless, the smoothening of the surface, when annealed using a MW, can be attributed to a step-bunching effect (i.e. aggregation of the ceramic steps) [40], sudden shrinkage and densification [38], and/or improved oxygen transport [41].

To elucidate the performance of our nanofibrillar YSZ in bone tissue engineering, hMSCs were grown on samples annealed at $5{ }^{\circ} \mathrm{C} / \mathrm{min}$ using both $\mathrm{CO}$ and $\mathrm{MW}$ heating. We chose these samples as they have the same temperature profile during annealing, grain sizes in the same order of magnitude and similar fiber diameter distributions, yet different surface roughness. Recent studies revealed the importance of nanoscale and submicron-sized topographic cues to modulate fundamental cell behavior, including proliferation, migration, and differentiation [42-44]. We hypothesized, therefore, that the nanofibrous structure and the different surface roughness of YSZ scaffolds may confer bioactive cues to an otherwise biologically inert ceramic material. Such topographical cues would ultimately result in varying cell morphology and, consequently, in different cell activities. The bioactivity of hMSCs on ceramic scaffolds was investigated by monitoring the cell viability, cellular morphology, cellular metabolism, alkaline phosphatase (ALP) activity, and gene expression in basic culture medium (BM) and osteogenic culture medium (OM) at days 7 and 14. Polystyrene tissue culture plates were used as a positive control (data not shown). The production of mineralized matrix was investigated after culturing cells-scaffold constructs in mineralization culture medium (MM) up to 28 days. Here, different culture media were chosen to investigate the influence of YSZ scaffolds on the osteogenesis potential of hMSCs in the presence or absence of osteoinducting soluble factors. BM is a maintenance medium that does not trigger the osteogenic differentiation of hMSCs $[30,45]$. On the other hand, OM induces osteogenic differentiation of hMSCs, whereas MM supports osteogenic differentiation and promotes the formation of mineralized matrix [30, 31].

To evaluate the viability of hMSCs on YSZ nanofibrillar scaffolds, a Live/Dead assay ${ }^{\circledR}$ was performed after 3 days of culture. The fluorescence microscopy images of both live and dead cells on scaffold are shown in Figure S4. All investigated scaffolds showed a 
high rate of viable cells, which indicates no cytotoxic effects derived from the developed YSZ nanofibrillar scaffolds.
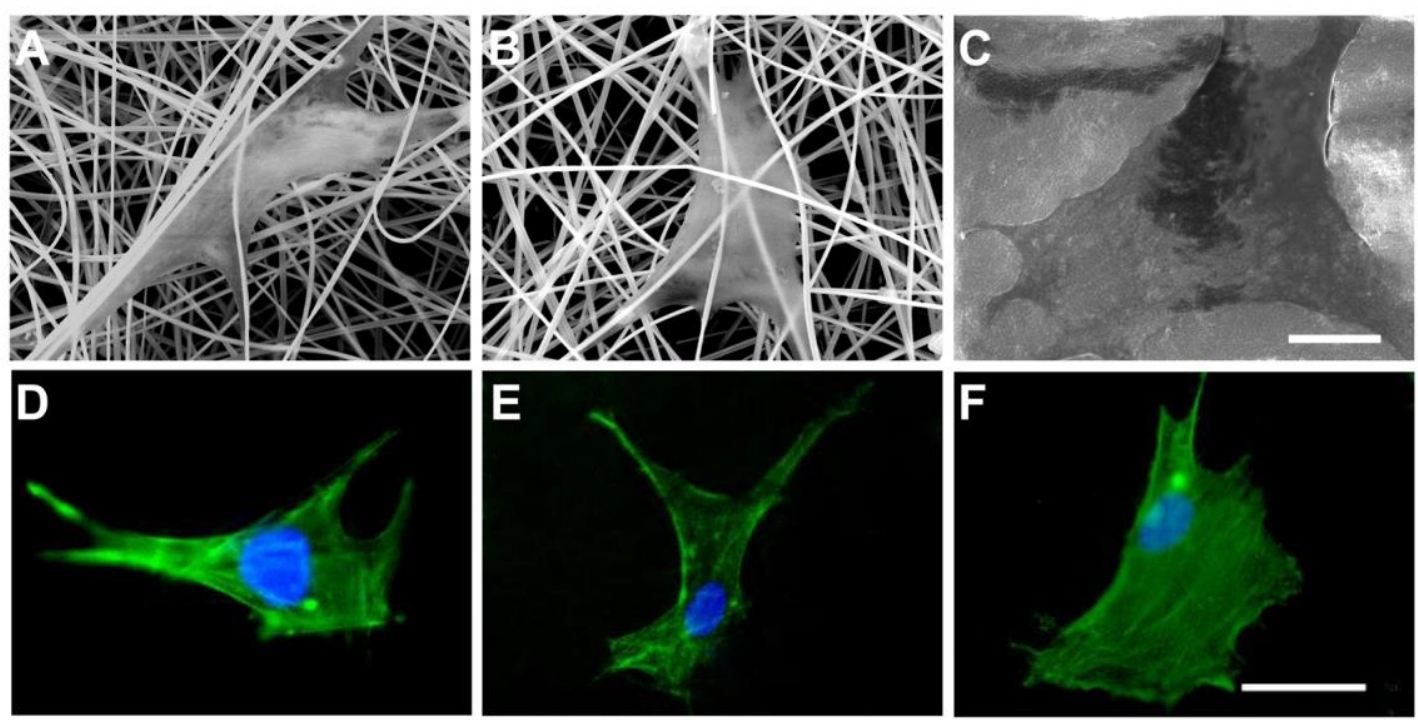

G
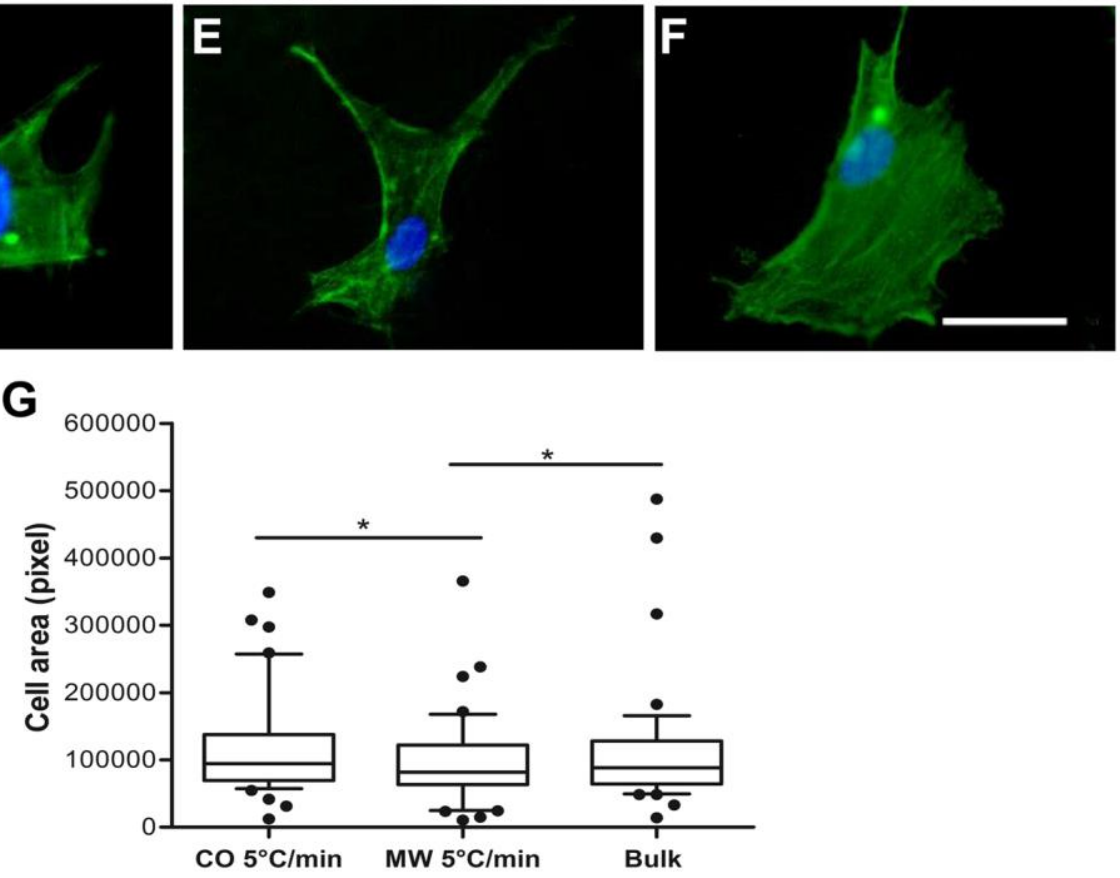

Figure 4. Characterization of cell morphology and actin cytoskeleton organization of hMSCs after 3 days of culture in BM. Representative SEM images of hMSCs on (A) microwave annealed (MW $5^{\circ} \mathrm{C} / \mathrm{min}$ ) nanofiber scaffolds, (B) conventional oven annealed $\left(\mathrm{CO} 5^{\circ} \mathrm{C} / \mathrm{min}\right)$ nanofiber scaffolds, and (C) YSZ bulk disk scaffolds. Representative images of actin cytoskeleton organization of hMSCs on (D) microwave annealed (MW $5^{\circ} \mathrm{C} / \mathrm{min}$ ) scaffolds, (E) conventional oven annealed ( $\mathrm{CO} 5^{\circ} \mathrm{C} / \mathrm{min}$ ) scaffolds, and (F) YSZ bulk disk scaffolds (actin filaments labeled with phalloidin are shown in green and nuclei labeled with DAPI are shown in blue). (G) Quantification of projected cell area on the investigated scaffolds. The statistical analysis was determined using two-tailed Student's t-test and ${ }^{*} p<0.05$ was considered as significant difference. The scale bar in (A-C) and (D-F) are10 $\mu \mathrm{m}$ and $50 \mu \mathrm{m}$ respectively.

The cell morphology on YSZ scaffolds was investigated by SEM (Figure 4A-C). The cell cytoskeleton was further assessed by staining with phalloidin for actin filaments (Figure 4D-F), and the average surface area for single cell was quantified (Figure 4G). Cells on $\mathrm{CO}$ annealed scaffolds had a higher cell area compared to MW annealed 
scaffolds, which could be correlated to the difference in surface roughness of the nanofibers. When comparing to that on bulk YSZ (having a roughness of $19 \pm 0.94 \mathrm{~nm}$ ), no significant difference in the average cell area was found possibly due to the combined effect of surface roughness and nanoscale fibrous structure.

Cell metabolic activity, which is indirectly related to cell viability, was determined using a PrestoBlue assay. The metabolic activity of hMSCs showed a similar profile in BM and OM (Figure S5). Bulk YSZ scaffolds had a significantly higher metabolic activity than all nanofibrous scaffolds, which could be due to the higher differentiation of hMSCs on nanofibrous scaffolds compared to bulk YSZ. Similar phenomena have been reported previously by Yuejun and his colleagues [46]. They found that hMSCs had higher metabolic activity on stiffer PDMS than the soft one while a higher differentiation activity of hMSCs were observed on soft PDMS compared to stiffer one. In addition, no significantly different metabolic activity was observed between $\mathrm{CO}$ and MW annealed scaffolds. This indicates that the surface roughness differences did not affect the cellular metabolism in the present work.

Polymerase chain reaction (PCR) was used to quantify the expression of a panel of osteogenic genes (Figure $5 \mathrm{C}$ and Figure S6). Gene expression of Runt-related transcription factor 2 (RUNX2) was significantly higher for $\mathrm{CO}$ and $\mathrm{MW}$ annealed scaffolds compared to bulk YSZ in both culture media. RUNX2 is considered as a focal point for integration of a variety of signals affecting the differentiation process of hMSCs into osteogenesis in the early stage, because it stimulates other downstream osteorelated genes such as osteopontin (OPN), osteocalcin (OCN), bone sialoprotein (BSP), and type I collagen[32]. Collagen type I alpha 1 (COL1A1) is fundamental for the development of the bone cell phenotype. It is actively expressed in the first proliferation period and then gradually down-regulated during subsequent osteoblast differentiation[47]. hMSCs cultured on annealed nanofibrous scaffolds showed higher expression of COL1A1 compared to bulk YSZ disks in both culture media. This is also associated with a significantly higher up-regulation of OCN and BSP on the nanofibrillar scaffolds in BM compared with bulk YSZ disks (Figure S6). Furthermore, the rougher meshes supported further enhanced OCN and BSP gene expression than the smoother ones, thus suggesting the possible role of surface topography on inducing hMSCs osteogenic differentiation, as also supported by other studies [48, 49]. OPN was only significantly up-regulated on nanofibrillar scaffolds at day 7 in $\mathrm{OM}$, while no beneficial 
effect of surface topography or nanofibrillar cues were observed for OCN or BSP in OM both at day 7 and at day 14 .

A

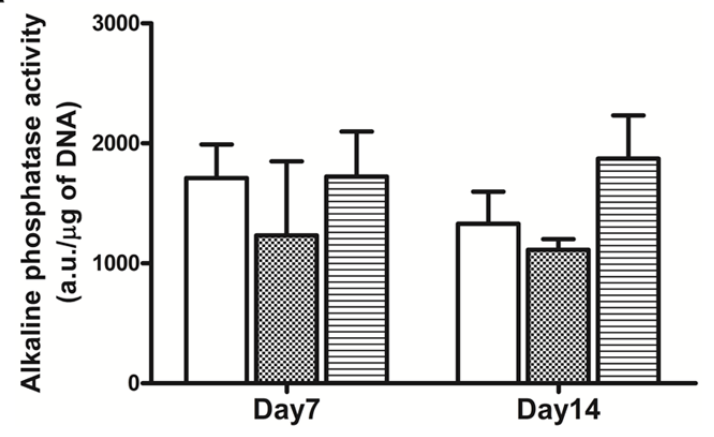

B

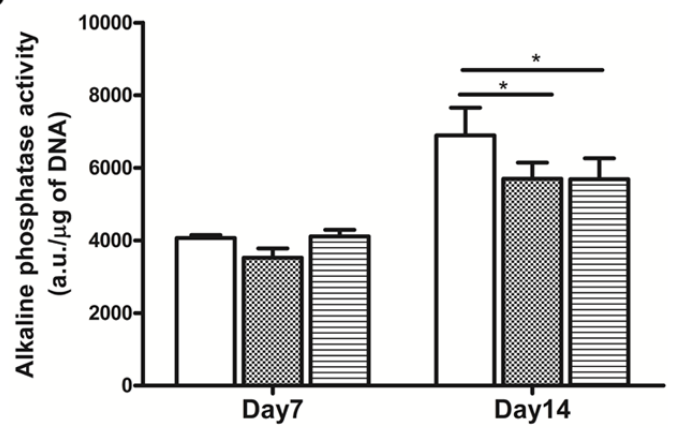

C
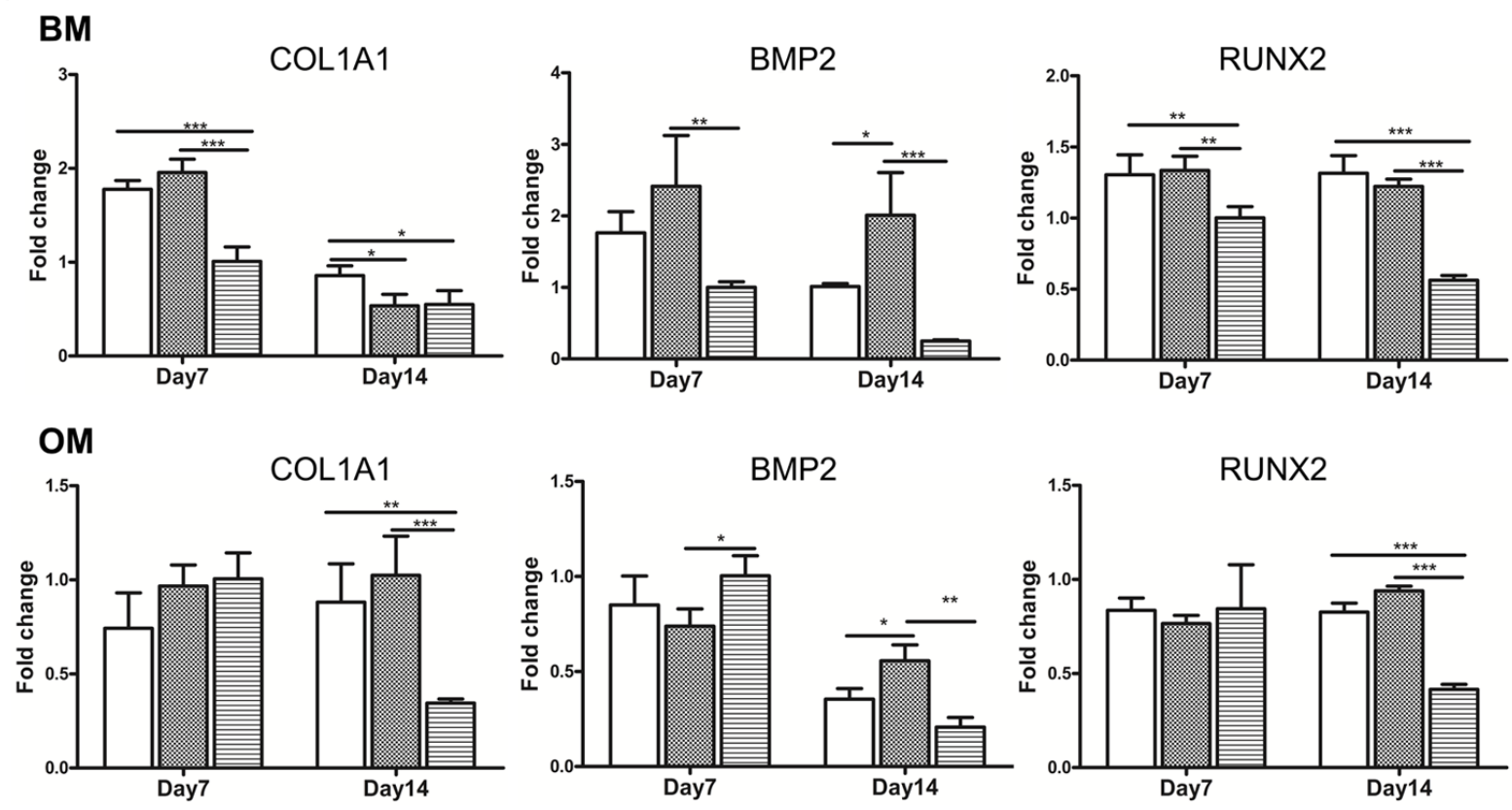

$\operatorname{CO} 5^{\circ} \mathrm{C} / \mathrm{min}$

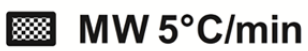

回 Bulk

Figure 5. ALP activity (A and B) and gene expression analysis (C) from hMSCs cultured on conventional oven annealed $\left(\mathrm{CO} 5^{\circ} \mathrm{C} / \mathrm{min}\right)$, microwave annealed $\left(\mathrm{MW} 5^{\circ} \mathrm{C} / \mathrm{min}\right)$ and $\mathrm{YSZ}$ bulk disk scaffolds. ALP activity of hMSCs cultured in BM (A) and OM (B) at day 7 and 14 (the final ALP activity level was shown after normalizing to DNA amount). (C) Gene expression analysis for COL1A1, BMP2, and RUNX2 at day 7 and 14 in $\mathrm{BM}$ and $\mathrm{OM}$ (the final gene expression level was presented after normalizing to day 7 bulk YSZ). Error bars represent standard deviation of the mean. ${ }^{*} p<0.05,{ }^{* *} p<0.01,{ }^{* * *} p<0.001$.

Since one of the route for hMSCs differentiation towards the osteogenic lineage passes through endochondral development $[50,51]$, the down-regulation of OCN and BSP on nanofibrous scaffolds in comparison with their bulk disks could be also attributed to a higher hypertrophic differentiation of hMSCs, and therefore a less mature osteogenic 
differentiation, on bulk disks due to their smooth surface properties in combination of medium stimuli. The smooth surface properties will affect the cell-to-material adhesion force and could have consequently influenced cell fate as well [52]. We also observed the expression of bone morphogenetic protein 2 (BMP2), which is known to be stimulating hMSCs along the osteogenic lineage [53]. BMP2 had a higher expression in all tested media on both nanofibrillar scaffolds compared to bulk YSZ except for day 7 in OM. Altogether, the ceramic nanofibrous scaffolds supported the osteogenic gene expression in both $\mathrm{BM}$ and $\mathrm{OM}$, thus suggesting a possible role of the nanofibrillar cues inherently built in the YSZ meshes in contributing to hMSCs osteogenic differentiation. In addition, the varying surface roughness resulting from the different fiber annealing processes showed a net beneficial effect in case of the OCN and BSP genes, which occurred only for the rougher nanofibrous scaffolds obtained by employing conventional heating. It is also worth to mention that there were different trends in OM compared to $\mathrm{BM}$, which might suggest that the differentiation of hMSCs in the present study was not only due to surface roughness but also other physical stimuli cues such as surface topography, porosity and physical cues associated to the nanoscale nature of the fibrillar meshes. Further studies should aim at clarifying the specific role of each of these material properties on cell differentiation, and the consequent osteoinductive nature of such nanofibrillar scaffolds, in addition to the osteoconductive properties here observed, both in vitro and in vivo.

At a protein level, the up-regulation of ALP activity is a common marker considered for the early stage of osteogenesis [32]. In BM, no significant differences were observed in ALP activity (Figure 5A). On the other hand, the ALP activity increased between day 7 and day 14 in $\mathrm{OM}$ and was significantly enhanced for $\mathrm{CO}$ annealed scaffolds compared to both MW annealed scaffolds and bulk YSZ (Figure 5B). These results indicate that the topographical properties of nanofibers, including larger surface area, higher porosity, and fibrous morphology, play a positive role in the osteogenic process of hMSCs. Similar results to our findings have been reported by Hae-Won Kim and colleagues who used bone marrow-derived stem cells to investigate the osteogenic potential of newly developed glass fibers [54]. Their results demonstrated that bioactive glass nanofibers showed a stronger ALP staining with respect to glass disks. 
A A $\quad \operatorname{Co~} 5^{\circ} \mathrm{C} / \mathrm{min}$

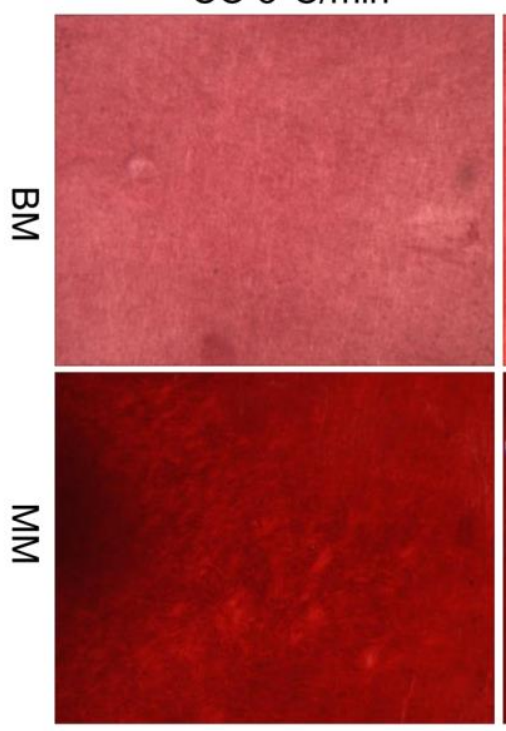

$\mathrm{MW} 5^{\circ} \mathrm{C} / \mathrm{min}$

Bulk
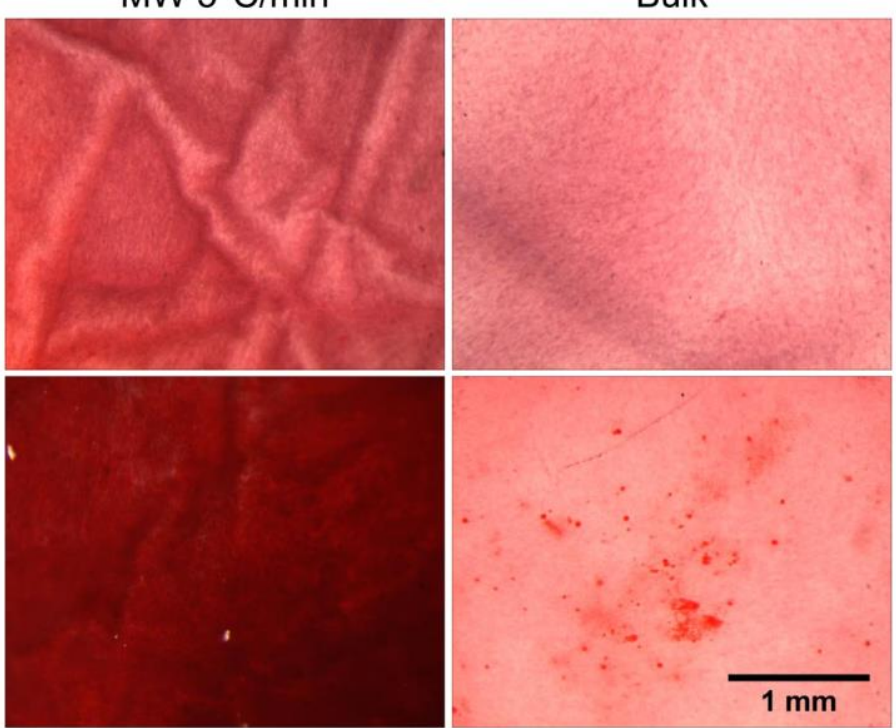

B

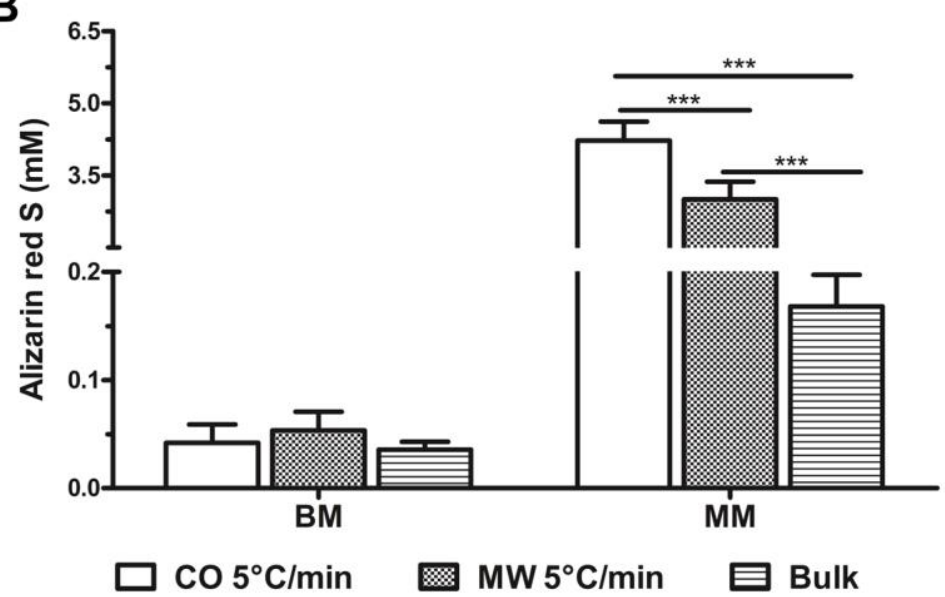

Figure 6. Calcium mineralization on conventional oven annealed $\left(\mathrm{CO} 5^{\circ} \mathrm{C} / \mathrm{min}\right)$, microwave annealed (MW $5^{\circ} \mathrm{C} / \mathrm{min}$ ), and YSZ bulk disk scaffolds. (A) The formation of mineralized extracellular matrix was stained with alizarin red $S$ after 28 days of culture in BM and $M M$ (the scale bar represents $1 \mathrm{~mm}$ ). (B) Quantitative analysis of the mineral deposits per scaffold by alizarin red $S$ staining at day 28 . Error bars represent standard deviation of the mean. ${ }^{* *} p<0.001$.

The ultimate deposition of a calcium-containing bone-like mineralized matrix is an index of extended osteogenic maturation of hMSCs. Mineralization was stained with alizarin red S after 28 days of culture (Figure 6 and Figure S7). When hMSCs were cultured on scaffolds in BM, little mineralized ECM was produced and no apparent differences were observed among the different scaffolds. In MM, a robust mineralized matrix was observed on bulk YSZ discs, while a strikingly significant increase in the amount of mineralized matrix was observed on the nanofibrous scaffolds. Quantification of the calcium deposits corroborated the staining results, showing significantly enhanced 
calcium deposition on both fiber scaffolds compared to the bulk YSZ discs (Figure 6B). Here, the rougher $\mathrm{CO}$ annealed scaffolds resulted in a further enhancement of calcium content compared to the MW annealed scaffolds. This results is similar to the finding by Takeuchi and his co-workers, who demonstrated that osteoblast show a higher rate of matrix mineralization on rougher titanium surfaces [55]. A recent study showed that hMSCs cultured on calcium phosphate cements had a mineral concentration of about 1 $\mathrm{mM}$ per scaffold at day 21[56]. Compared to this study, our nanofibers annealed in a conventional oven had almost 3 folds higher mineral concentration. However, when comparing to other studies, care should be taken as different cell donors, time point and cell seeding density have been used.

\subsection{Conclusions}

In summary, new flexible nanofibrous YSZ scaffolds that display multiscale mechanical properties were successfully fabricated by electrospinning. Their surface roughness can be tailored by employing different heat treatment method. Our results demonstrate the osteogenic differentiation and mineralization of seeded human mesenchymal stromal cells was supported by the nanofibrous structure of YSZ scaffolds, in contrast to the wellknown bioinert behavior of bulk YSZ. These flexible scaffolds can therefore present an appealing alternative to current brittle ceramics for bone regeneration applications. 


\section{References}

[1] E. Champion, Sintering of calcium phosphate bioceramics, Acta Biomaterialia 9(4) (2013) 5855-5875.

[2] S.M. Best, A.E. Porter, E.S. Thian, J. Huang, Bioceramics: Past, present and for the future, Journal of the European Ceramic Society 28(7)(2008)1319-1327.

[3] S.M. Zakaria, S.H. Sharif Zein, M.R. Othman, F. Yang, J.A. Jansen, Nanophase Hydroxyapatite as a biomaterial in advanced hard tissue engineering: A review, Tissue Engineering - Part B: Reviews 19(5)(2013)431-441.

[4] J.R. Woodard, A.J. Hilldore, S.K. Lan, C.J. Park, A.W. Morgan, J.A.C. Eurell, S.G. Clark, M.B. Wheeler, R.D. Jamison, A.J. Wagoner Johnson, The mechanical properties and osteoconductivity of hydroxyapatite bone scaffolds with multi-scale porosity, Biomaterials 28(1)(2007) 45-54.

[5] D.W. Hutmacher, Scaffolds in tissue engineering bone and cartilage, Biomaterials 21(24)(2000)25292543.

[6] H.-W. Kim, J.C. Knowles, H.-E. Kim, Hydroxyapatite/poly(ع-caprolactone) composite coatings on hydroxyapatite porous bone scaffold for drug delivery, Biomaterials 25(7-8) (2004) 1279-1287.

[7] V. Karageorgiou, D. Kaplan, Porosity of 3D biomaterial scaffolds and osteogenesis, Biomaterials 26(27)(2005) 5474-5491.

[8] D.W. Hutmacher, T. Schantz, I. Zein, K.W. Ng, S.H. Teoh, K.C. Tan, Mechanical properties and cell cultural response of polycaprolactone scaffolds designed and fabricated via fused deposition modeling, Journal of Biomedical Materials Research 55(2)(2001) 203-216.

[9] C. Piconi, G. Maccauro, Zirconia as a ceramic biomaterial, Biomaterials 20(1) (1999)1-25.

[10] I. Denry, J.R. Kelly, State of the art of zirconia for dental applications, Dental Materials 24(3)(2008) 299-307.

[11] K.A. Hing, B. Annaz, S. Saeed, P.A. Revell, T. Buckland, Microporosity enhances bioactivity of synthetic bone graft substitutes, Journal of Materials Science: Materials in Medicine 16(5)(2005)467-475.

[12] L.R. Meza, S. Das, J.R. Greer, Strong, lightweight, and recoverable three-dimensional ceramic nanolattices, Science 345(6202)(2014)1322-1326.

[13] S.-J. Park, G. Chase, K.-U. Jeong, H. Kim, Mechanical properties of titania nanofiber mats fabricated by electrospinning of sol-gel precursor, Journal of Sol-Gel Science and Technology 54(2)(2010)188-194.

[14] M. Guo, B. Ding, X. Li, X. Wang, J. Yu, M. Wang, Amphiphobic Nanofibrous Silica Mats with Flexible and High-Heat-Resistant Properties, The Journal of Physical Chemistry C 114(2)(2009) 916-921.

[15] A. Biswas, H. Park, W.M. Sigmund, Flexible ceramic nanofibermat electrospun from TiO2-SiO2 aqueous sol, Ceramics International 38(1)(2012) 883-886.

[16] M. Xi, X. Wang, Y. Zhao, Q. Feng, F. Zheng, Z. Zhu, H. Fong, Mechanically flexible hybrid mat consisting of $\mathrm{TiO}_{2}$ and $\mathrm{SiO}_{2}$ nanofibers electrospun via dual spinnerets for photo-detector, Materials Letters 120(0)(2014)219-223.

[17] Y. Si, X. Tang, J. Ge, S. Yang, M. El-Newehy, S.S. Al-Deyab, J. Yu, B. Ding, In situ synthesis of flexible magnetic [gamma]-Fe2O3@SiO2 nanofibrous membranes, Nanoscale 6(4)(2014)2102-2105.

[18] Y. Chen, X. Mao, H. Shan, J. Yang, H. Wang, S. Chen, F. Tian, J. Yu, B. Ding, Free-standing zirconia nanofibrous membranes with robust flexibility for corrosive liquid filtration, RSC Advances 4(6)(2014)27562763. 
[19] X. Mao, Y. Si, Y. Chen, L. Yang, F. Zhao, B. Ding, J. Yu, Silica nanofibrous membranes with robust flexibility and thermal stability for high-efficiency fine particulate filtration, RSC Advances 2(32)(2012)1221612223.

[20] R. Wang, J. Guo, D. Chen, Y.-E. Miao, J. Pan, W.W. Tjiu, T. Liu, "Tube brush" like ZnO/SiO2 hybrid to construct a flexible membrane with enhanced photocatalytic properties and recycling ability, Journal of Materials Chemistry 21(48)(2011)19375-19380.

[21] H. Chen, J. Huang, J. Yu, S. Liu, P. Gu, Electrospun chitosan-graft-poly ( $\varepsilon$-caprolactone)/poly ( $\varepsilon$ caprolactone) cationic nanofibrous mats as potential scaffolds for skin tissue engineering, International journal of biological macromolecules 48(1) (2011)13-19.

[22] H. Chen, X. Fan, J. Xia, P. Chen, X. Zhou, J. Huang, J. Yu, P. Gu, Electrospun chitosan-graft-poly ( $\varepsilon-$ caprolactone)/poly ( $\varepsilon$-caprolactone) nanofibrous scaffolds for retinal tissue engineering, International journal of nanomedicine 6 (2011) 453.

[23] R.J. Wade, E.J. Bassin, C.B. Rodell, J.A. Burdick, Protease-degradable electrospun fibrous hydrogels, Nature communications 6 (2015).

[24] F. Zamani, M. Amani-Tehran, M. Latifi, M.A. Shokrgozar, The influence of surface nanoroughness of electrospun PLGA nanofibrous scaffold on nerve cell adhesion and proliferation, Journal of Materials Science: Materials in Medicine 24(6)(2013)1551-1560.

[25] A.-M. Azad, Fabrication of yttria-stabilized zirconia nanofibers by electrospinning, Materials Letters 60(1)(2006)67-72.

[26] S. Xu, Y. Shi, S.G. Kim, Fabrication and mechanical property of nano piezoelectric fibres, Nanotechnology 17(17)(2006)4497-4501.

[27] S.-H. Lee, C. Tekmen, W.M. Sigmund, Three-point bending of electrospun TiO2 nanofibers, Materials Science and Engineering: A 398(1-2)(2005)77-81.

[28] C.M. DiGirolamo, D. Stokes, D. Colter, D.G. Phinney, R. Class, D.J. Prockop, Propagation and senescence of human marrow stromal cells in culture: a simple colony-forming assay identifies samples with the greatest potential to propagate and differentiate, British Journal of Haematology 107(2)(1999) 275281.

[29] D.G. Phinney, G. Kopen, W. Righter, S. Webster, N. Tremain, D.J. Prockop, Donor variation in the growth properties and osteogenic potential of human marrow stromal cells, Journal of Cellular Biochemistry 75(3)(1999) 424-436.

[30] A. Nandakumar, L. Yang, P. Habibovic, C. van Blitterswijk, Calcium phosphate coated electrospun fiber matrices as scaffolds for bone tissue engineering, Langmuir 26(10)(2009)7380-7387.

[31] A. Nandakumar, A. Barradas, J. de Boer, L. Moroni, C. van Blitterswijk, P. Habibovic, Combining technologies to create bioactive hybrid scaffolds for bone tissue engineering, Biomatter 3(2)(2013).

[32] A.K. Gaharwar, S.M. Mihaila, A. Swami, A. Patel, S. Sant, R.L. Reis, A.P. Marques, M.E. Gomes, A. Khademhosseini, Bioactive silicate nanoplatelets for osteogenic differentiation of human mesenchymal stem cells, Advanced Materials 25(24) (2013)3329-3336.

[33] C.A. Gregory, W.G. Gunn, A. Peister, D.J. Prockop, An Alizarin red-based assay of mineralization by adherent cells in culture: comparison with cetylpyridinium chloride extraction, Analytical biochemistry 329(1) (2004)77-84. 
[34] A. Biswas, H. Park, W.M. Sigmund, Flexible ceramic nanofibermat electrospun from TiO2-SiO 2 aqueous sol, Ceramics International 38(1)(2012) 883-886.

[35] J.W. Adams, R. Ruh, K. Mazdiyasni, Young's Modulus, Flexural Strength, and Fracture of YttriaStabilized Zirconia versus Temperature, Journal of the American Ceramic Society 80(4)(1997) 903-908.

[36] R. Chaim, M. Hefetz, Effect of grain size on elastic modulus and hardness of nanocrystalline ZrO2-3 wt\% $\mathrm{Y}_{2} \mathrm{O}_{3}$ ceramic, Journal of materials science 39(9)(2004) 3057-3061.

[37] S. Ramesh, S. Meenaloshini, C. Tan, W.K. Chew, W. Teng, Effect of manganese oxide on the sintered properties and low temperature degradation of Y-TZP ceramics, Ceramics International 34(7)(2008)16031608.

[38] J. Wang, J. Binner, B. Vaidhyanathan, N. Joomun, J. Kilner, G. Dimitrakis, T.E. Cross, Evidence for the microwave effect during hybrid sintering, Journal of the American Ceramic Society 89(6)(2006)1977-1984.

[39] Z. Xie, J. Yang, X. Huang, Y. Huang, Microwave processing and properties of ceramics with different dielectric loss, Journal of the European Ceramic Society 19(3) (1999)381-387.

[40] S.G. Sundaresan, M.V. Rao, Y.-I. Tian, M.C. Ridgway, J.A. Schreifels, J.J. Kopanski, Ultrahightemperature microwave annealing of $\mathrm{Al}_{+-}$and $\mathrm{P}+$-implanted $4 \mathrm{H}-\mathrm{SiC}$, Journal of Applied Physics 101(7)(2007) .

[41] A. Goldstein, N. Travitzky, A. Singurindy, M. Kravchik, Direct microwave sintering of yttria-stabilized zirconia at 2.45\&\#xa0;GHz, Journal of the European Ceramic Society 19(12)(1999)2067-2072.

[42] S. Watari, K. Hayashi, J.A. Wood, P. Russell, P.F. Nealey, C.J. Murphy, D.C. Genetos, Modulation of osteogenic differentiation in hMSCs cells by submicron topographically-patterned ridges and grooves, Biomaterials 33(1)(2012) 128-136.

[43] S.A. Pot, S.J. Liliensiek, K.E. Myrna, E. Bentley, J.V. Jester, P.F. Nealey, C.J. Murphy, Nanoscale topography-induced modulation of fundamental cell behaviors of rabbit corneal keratocytes, fibroblasts, and myofibroblasts, Investigative ophthalmology \& visual science 51(3)(2010)1373-1381.

[44] L.E. McNamara, R.J. McMurray, M.J. Biggs, F. Kantawong, R.O. Oreffo, M.J. Dalby, Nanotopographical control of stem cell differentiation, Journal of tissue engineering 1(1) (2010) 120623.

[45] H. Fernandes, A. Mentink, R. Bank, R. Stoop, C. van Blitterswijk, J. de Boer, Endogenous collagen influences differentiation of human multipotent mesenchymal stromal cells, Tissue Engineering Part $A$ 16(5)(2010) 1693-1702.

[46] Y.J. Chuah, Y. Zhang, Y. Wu, N.V. Menon, G.H. Goh, A.C. Lee, V. Chan, Y. Zhang, Y. Kang, Combinatorial effect of substratum properties on mesenchymal stem cell sheet engineering and subsequent multi-lineage differentiation, Acta Biomaterialia (2015).

[47] A. Polini, D. Pisignano, M. Parodi, R. Quarto, S. Scaglione, Osteoinduction of human mesenchymal stem cells by bioactive composite scaffolds without supplemental osteogenic growth factors, PloS one 6(10) (2011) e26211.

[48] H. Yuan, H. Fernandes, P. Habibovic, J. de Boer, A.M. Barradas, A. de Ruiter, W.R. Walsh, C.A. van Blitterswijk, J.D. de Bruijn, Osteoinductive ceramics as a synthetic alternative to autologous bone grafting, Proceedings of the National Academy of Sciences 107(31) (2010) 13614-13619. 
[49] O. Gauthier, J.-M. Bouler, E. Aguado, P. Pilet, G. Daculsi, Macroporous biphasic calcium phosphate ceramics: influence of macropore diameter and macroporosity percentage on bone ingrowth, Biomaterials 19(1) (1998) 133-139.

[50] C. Scotti, B. Tonnarelli, A. Papadimitropoulos, A. Scherberich, S. Schaeren, A. Schauerte, J. LopezRios, R. Zeller, A. Barbero, I. Martin, Recapitulation of endochondral bone formation using human adult mesenchymal stem cells as a paradigm for developmental engineering, Proceedings of the National Academy of Sciences 107(16) (2010) 7251-7256.

[51] L. Gerstenfeld, F. Shapiro, Expression of bone-specific genes by hypertrophic chondrocytes: Implications of the complex functions of the hypertrophic chondrocyte during endochondral bone development, Journal of cellular biochemistry 62(1) (1996) 1-9.

[52] F. Kitagawa, S. Takei, T. Imaizumi, Y. Tabata, Chondrogenic differentiation of immortalized human mesenchymal stem cells on zirconia microwell substrata, Tissue Engineering Part C: Methods 19(6)(2012) 438-448.

[53] C.M. Curtin, G.M. Cunniffe, F.G. Lyons, K. Bessho, G.R. Dickson, G.P. Duffy, F.J. O'Brien, Innovative Collagen Nano-Hydroxyapatite Scaffolds Offer a Highly Efficient Non-Viral Gene Delivery Platform for Stem Cell-Mediated Bone Formation, Advanced Materials 24(6) (2012) 749-754.

[54] H.W. Kim, H.E. Kim, J.C. Knowles, Production and Potential of Bioactive Glass Nanofibers as a NextGeneration Biomaterial, Advanced Functional Materials 16(12) (2006) 1529-1535.

[55] K. Takeuchi, L. Saruwatari, H.K. Nakamura, J.M. Yang, T. Ogawa, Enhanced intrinsic biomechanical properties of osteoblastic mineralized tissue on roughened titanium surface, Journal of Biomedical Materials Research Part A 72(3) (2005) 296-305.

[56] M. Tang, W. Chen, J. Liu, M.D. Weir, L. Cheng, H.H. Xu, Human induced pluripotent stem cell-derived mesenchymal stem cell seeding on calcium phosphate scaffold for bone regeneration, Tissue Engineering Part A 20(7-8) (2014) 1295-1305. 


\section{Supplementary Information}

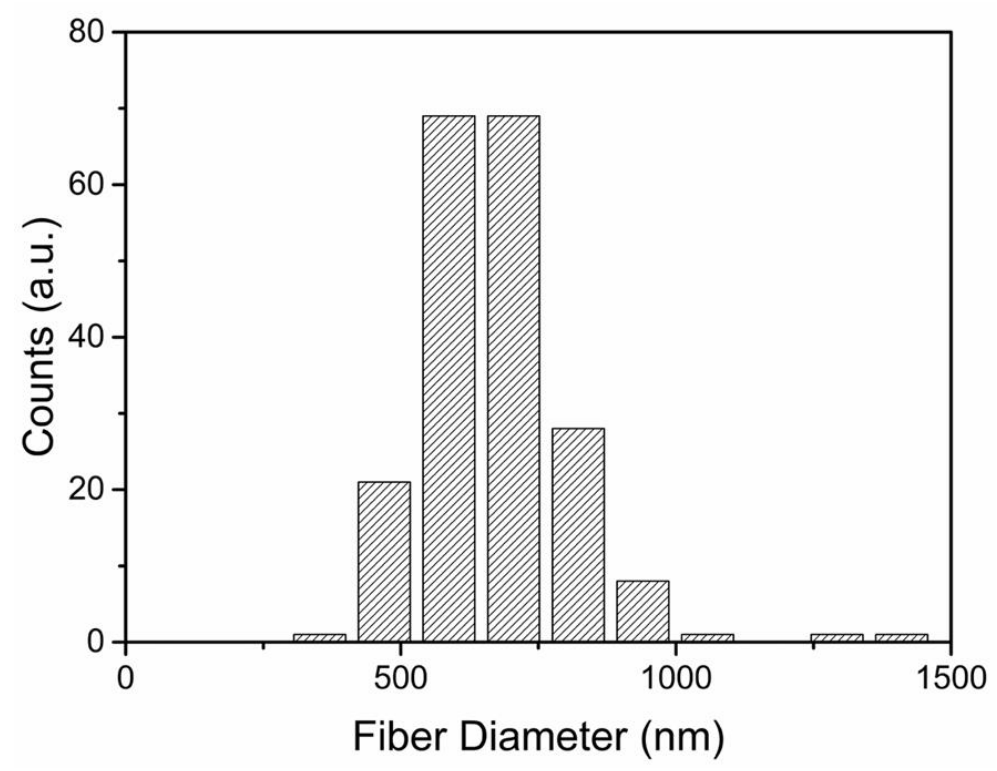

Figure S1. Fiber diameter distribution. Frequency distribution of fiber diameter as calculated from the SEM pictures.

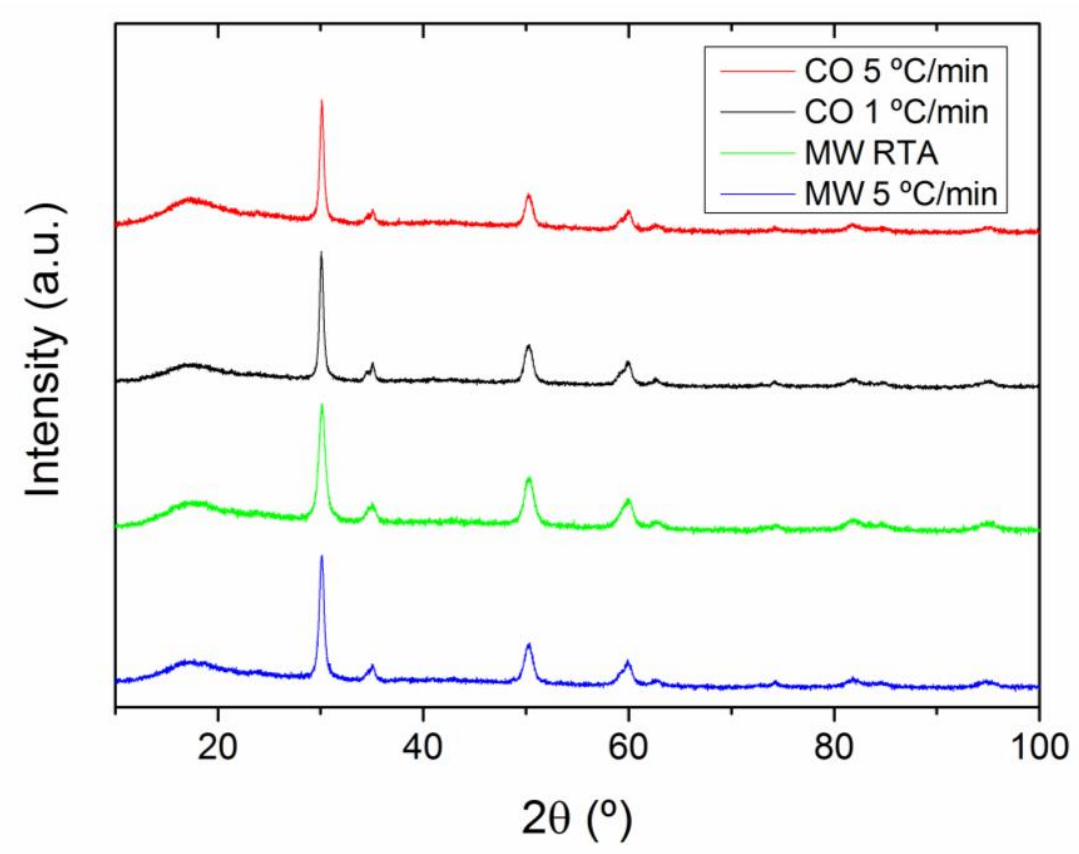

Figure S2. XRD patterns of the nanofiber mats for different annealing conditions. The XRD patterns match the crystal structure of $3 \%$ mol yttria stabilized zirconia (ICDB 01-070-4426). CO = conventional oven; MW = microwave oven; MW RTA= rapid thermal annealing in microwave oven. It can be seen that the crystallinity of the samples is the same within experimental error. 

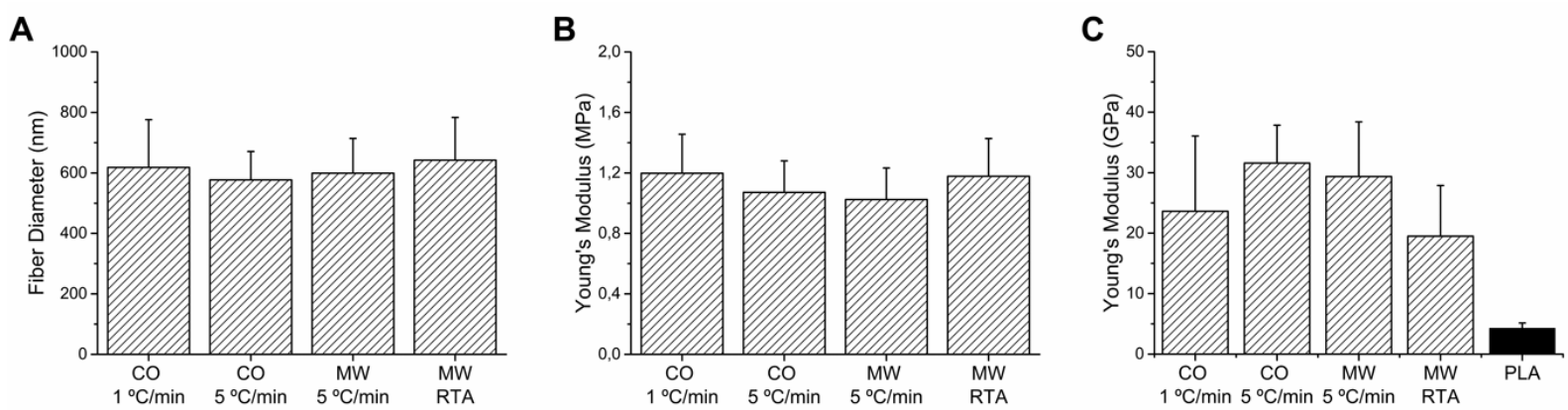

Figure S3. The average fiber diameter and Young's moduli. (A) Fiber diameter calculated from SEM images as a function of annealing procedure; (B) Young's moduli measured by microindentation; (C) Young's moduli measured by a 3 point bending test of single fibers using an AFM. Polylactic acid (PLA) nanofibers were used as reference to verify the accuracy of the measurement. Error bars represent standard deviation of the mean. The statistical significance was determined using a one way ANOVA test, where ${ }^{*} \mathrm{p}<0.05$. $\mathrm{CO}=$ conventional oven; $\mathrm{MW}=$ microwave oven; MW RTA= rapid thermal annealing in microwave oven.
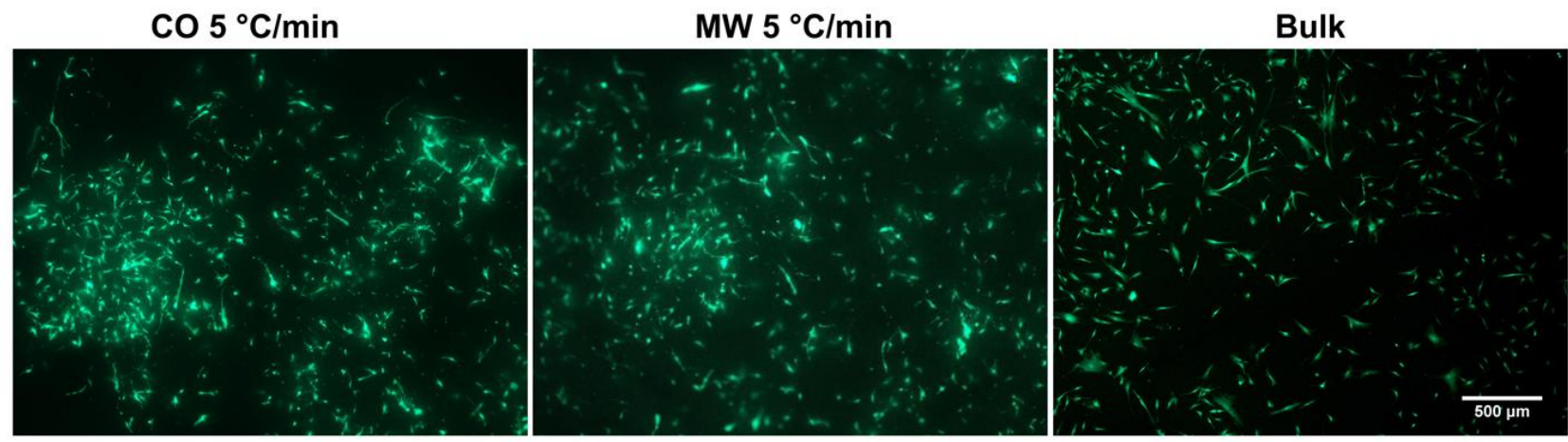

Figure S4. Live \& dead assay. hMSCs viability on ceramic scaffolds at day 3 in BM. Live and dead cells are represented by green and red fluorescence, respectively. The scale bar is $500 \mu \mathrm{m}$. CO = conventional oven; $\mathrm{MW}=$ microwave oven; $\mathrm{BM}=$ basic medium . 
A

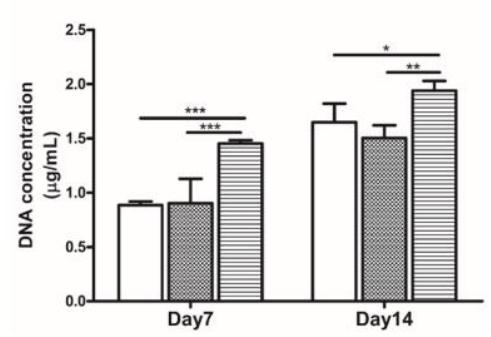

C

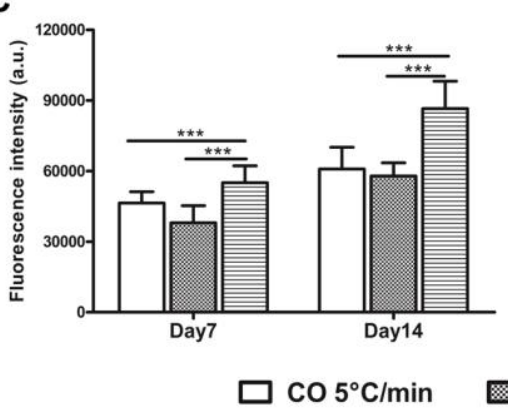

B

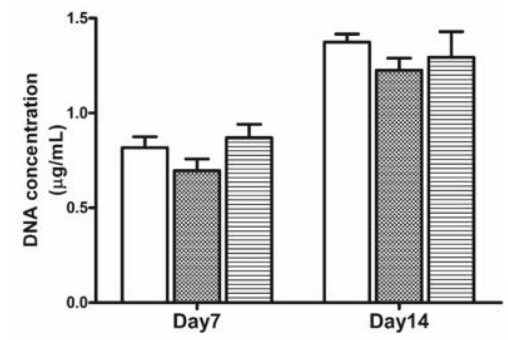

D

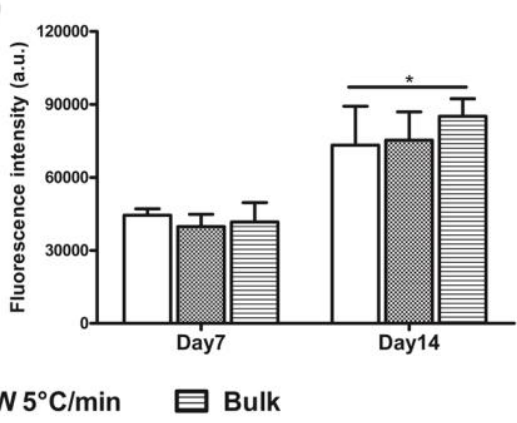

Figure S5. DNA amounts (A-B) and metabolic activity (C-D) of hMSCs cultured on ceramic scaffolds in (A and $C) B M$ and $(B$ and $D) O M$ at day 7 and 14. Error bars represent standard deviation of the mean. ${ }^{*} p<0.05$, ${ }^{* * *} \mathrm{p}<0.001$. $\mathrm{CO}=$ conventional oven; $\mathrm{MW}=$ microwave oven; $\mathrm{BM}=$ basic medium; $\mathrm{OM}=$ osteogenic medium.
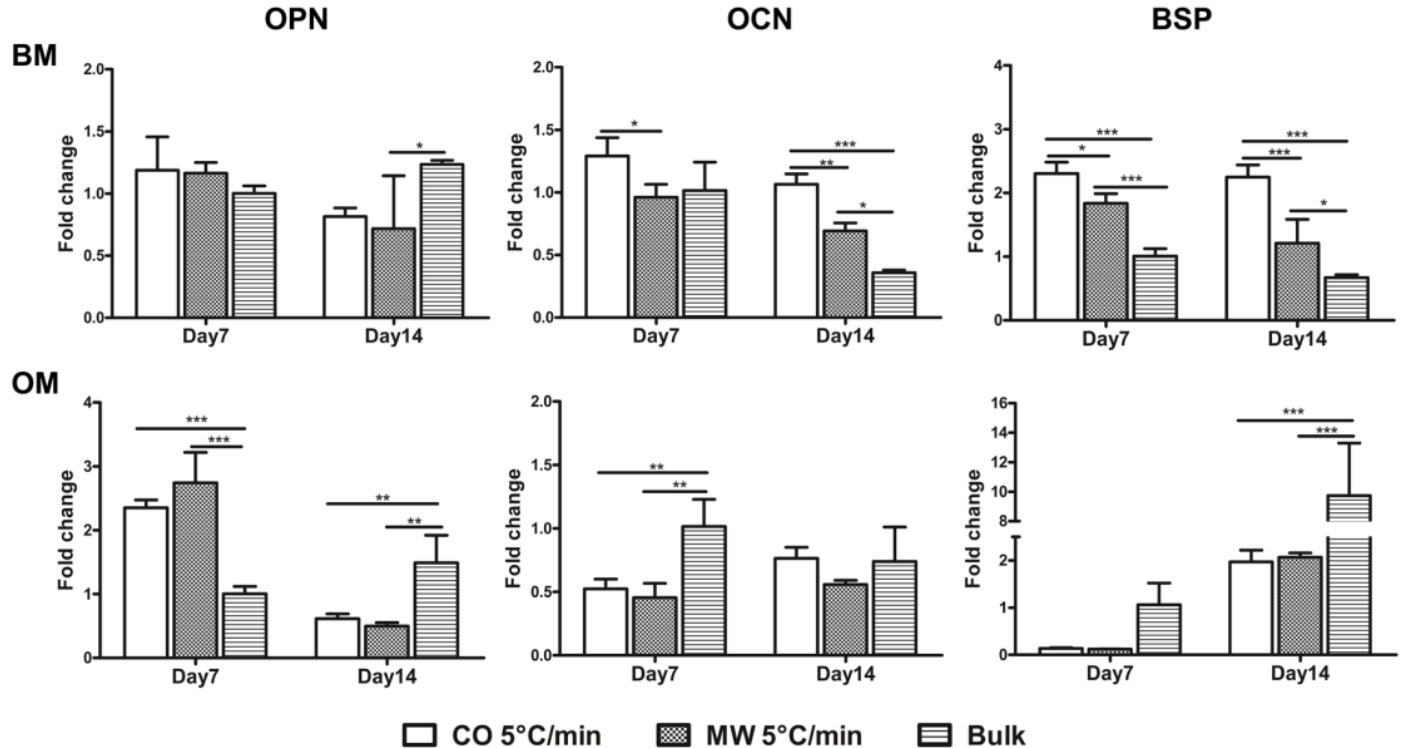

Figure S6. The expressions of osteopontin (OPN), osteocalcin (OCN), and bone sialoprotein (BSP) in hMSCs cultured on scaffolds in BM and OM. The results were presented after normalizing to day 7 bulk YSZ discs in BM and OM, respectively. Error bars represent standard deviation of the mean. ${ }^{*} p<0.05,{ }^{* *} p<0.01$, 
${ }^{* * *} \mathrm{p}<0.001 . \mathrm{CO}=$ conventional oven; $\mathrm{MW}=$ microwave oven; $\mathrm{BM}=$ basic medium; $\mathrm{OM}=$ osteogenic medium.

A

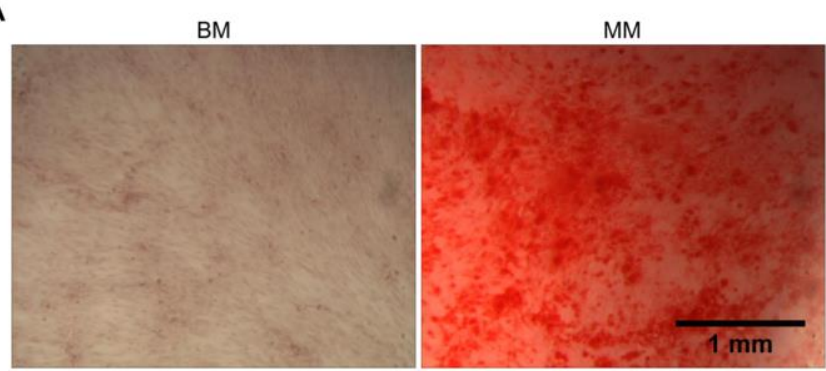

B

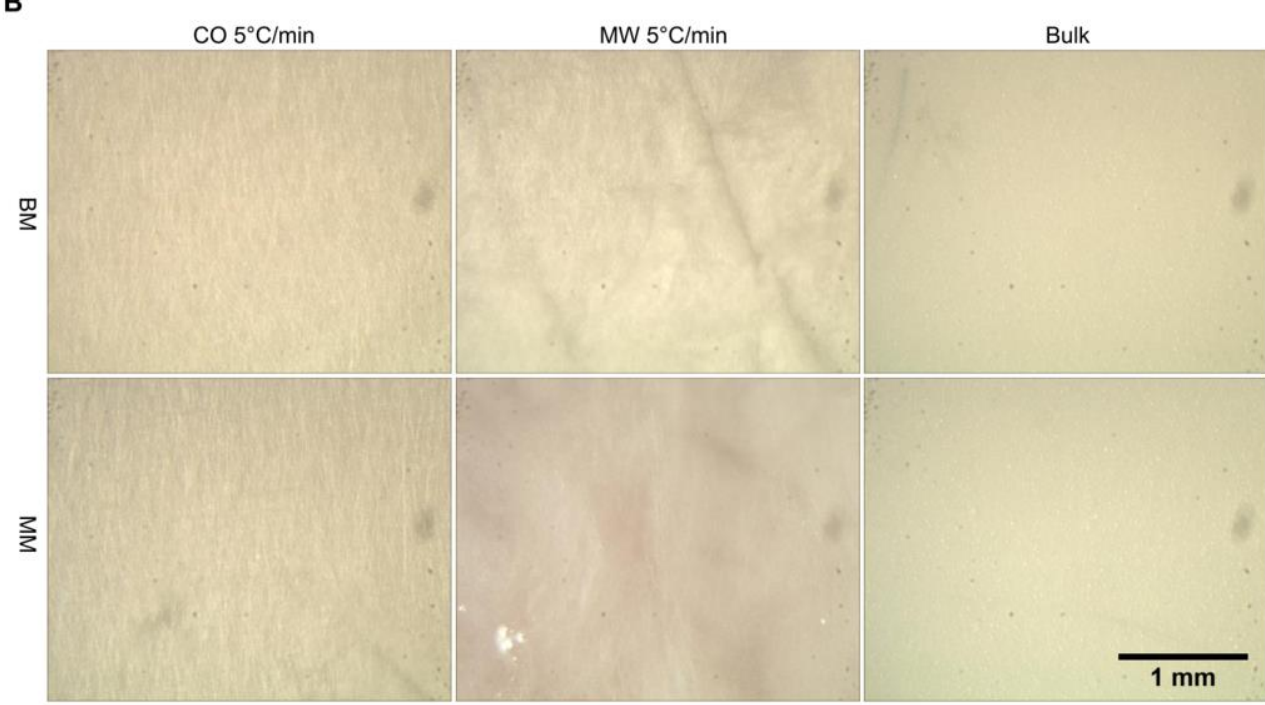

Figure S7. Calcium deposition test. (A) hMSCs cultured in a tissue culture plate as a control experiment, and stained for alizarin red $S$ after 28 days culture in BM and MM; (B) The ceramic scaffolds without seeding hMSCs incubated in BM and MM for 28 days do not stain for alizarin red S. CO = conventional oven; $\mathrm{MW}=$ microwave oven; $\mathrm{BM}=$ basic medium; $\mathrm{MM}=$ mineralization medium.

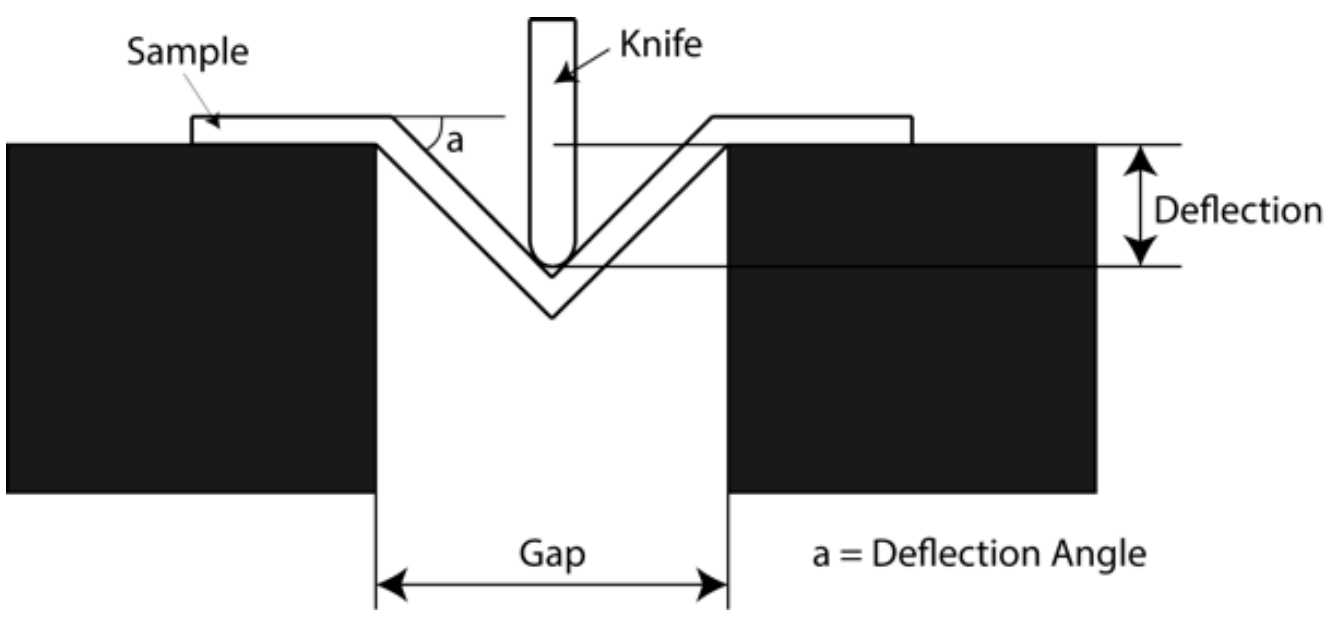

Figure S8. Schematic representation of the 3 point bending fatigue test. 


\section{Chapter 4}

\section{Tailoring Surface Nanoroughness of Electrospun Scaffolds for Skeletal Tissue Engineering}

Honglin Chen ${ }^{\mathrm{a}, \mathrm{b}}$, Xiaobin Huang ${ }^{\mathrm{b}}$, Minmin Zhang ${ }^{\mathrm{c}}$, Febriyani Damanik ${ }^{\mathrm{a}}$ Mattew B. Baker ${ }^{\mathrm{a}}$, Anne Leferink $^{\mathrm{a}}$, Huipin Yuan ${ }^{\mathrm{a}}$, Roman Truckenmüller ${ }^{\mathrm{a}}$, Clemens van Blitterswijk ${ }^{\mathrm{a}}$, Lorenzo Moroni ${ }^{\mathrm{a}}$

aMERLN Institute for Technology Inspired Regenerative Medicine, 6200 MD Maastricht, The Netherlands

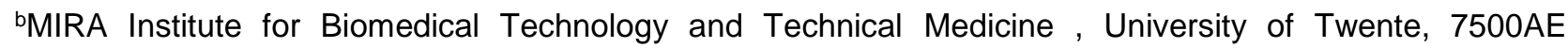
Enschede, The Netherlands

cMESA+ Research Institute, University of Twente, 7500 AE Enschede, The Netherlands 


\begin{abstract}
:
Electrospun scaffolds provide a promising approach for tissue engineering as they mimic the physical properties of the extracellular matrix. Previous studies have demonstrated that electrospun scaffolds with porous features on the surface of single fibers, enhanced cellular attachment and proliferation. Yet, little is known about the effect of such topographical cues on cellular differentiation. Here, we aimed at investigating the influence of surface roughness of electrospun scaffolds on skeletal differentiation of human mesenchymal stromal cells (hMSCs). Scanning electron microscopy (SEM) and atomic force microscopy (AFM) analysis showed that the surface nanoroughness of fibers was successfully regulated via humidity control of the electrospinning environment.. Gene expression analysis revealed that a higher surface roughness $(\mathrm{Ra}=71.0 \mathrm{~nm})$ supported more induction of osteogenic genes such as osteopontin (OPN), bone morphogenetic protein 2 (BMP2), and runt-related transcription factor 2 (RUNX2), while a lower surface roughness $(\mathrm{Ra}=14.3 \mathrm{~nm}$ ) demonstrated higher expression of other osteogenic genes including bone sialoprotein (BSP), collagen, type I, alpha 1(COL1A1) and osteocalcin (OCN). Interestingly, a lower surface roughness $(\mathrm{Ra}=14.3 \mathrm{~nm})$ better supported chondrogenic gene expression of hMSCs at day 7 compared to higher surface roughness $(\mathrm{Ra}=71.0)$. Taken together, modulating surface roughness of 3D scaffolds appears to be significant factor in scaffold design for the control of skeletal differentiation of hMSCs.
\end{abstract}

\title{
Key words:
}

Surface roughness, mesenchymal stromal cells, osteogenisis, chondrogenisis, 3D scaffold 


\subsection{Introduction}

Human mesenchymal stromal cells (hMSCs) are considered as a promising candidate cell source for skeletal tissue engineering and regeneration due to their low immunogenic reaction in allogeneic hosts and multilineage differentiation potential including the chondrogenic, adipogenic and osteogenic lineages [1-4]. Moreover, hMSCs are an easy accessible cell source [5]. Intrinsic properties of the extracellular matrix (ECM), such as chemistry, and stiffness, have been demonstrated to regulate stem cell differentiation. It also has been proved that tailoring surface topography serves as one of the key roles regulating stem cell differentiation [6,7]. Dalby and his co-workers cultured hMSCs on poly(methyl methacrylate) substrate with varying ordered nanopits [8] and found that semiordered nanopits $(120 \mathrm{~nm}$ diameter, $100 \mathrm{~nm}$ deep, absolute or average $300 \mathrm{~nm}$ centrecentre spacing) induced the expression of bone specific proteins in hMSCs in contrast to completely ordered or random nanopits. In the work by Nuno et al., hMSCs were cultured on polycaprolactone substrates displaying a gradient in surface roughness [6]. Specific roughness $\quad(\mathrm{Ra} \sim 2.1-3.1 \mu \mathrm{m} ; \mathrm{RS} m \sim 71.1-48.1 \mu \mathrm{m})$ steered faster osteogenic commitment and stronger osteogenic expression compared to tissue culture polystyrene.

Recently, electrospun fibers have been intensively utilized as scaffolds in tissue engineering owing to their fibrous structure mimicking the physical dimensions of natural fibrous extracellular matrix [9-12]. Moreover, these fibers can be easily fabricated and modified [13]. The morphology of electrospun fibrous structure can be subdivided into the morphologies of single fiber and fibrous mesh [14]. The modification of the morphology of single fibers could be achieved via many approaches including varying collector temperature [15], solvent and relative humidity [9, 16], or by post-processing methods such as nanoimprint lithography [13], surface graft polymerization [17], physical coating or blending [18], wet chemical etching methods [19], and gas plasma [20]. Among these methods, varying the solvent and relative humidity has multiple superior advantages to other methods, since (i) it is a simple and direct process without any post-electrospinning treatment compared to nanoimprinting [13], wet chemical etching [19] and plasma [21]; (ii) it shows higher flexibility in controlling the degree of roughness than varying the collector temperature [15] and physical coating or blending [22]; (iii) it results in homogeneous treatment of each single fiber in contrast to nanoimprinting [13] and gas plasma [23] which can modify only localized surface areas of fibrous meshes. Therefore, this approach has been employed to produce electrospun scaffolds with nanoporous features on their single fibers for a wide range of applications, such as drug delivery, filtration, and tissue 
engineering $[9,15]$. Previous studies have been demonstrated that the modification of the morphology of single fibers in electrospun scaffolds can directly affect cellular response [24-28]. We previously demonstrated that hMSCs cultured on nano-porous $10 \mu \mathrm{m}$ fiber scaffolds had higher proliferation rates than hMSCs cultured on smooth $10 \mu \mathrm{m}$ fiber scaffolds [24]. In addition, these hMSC cells were found to aggregate on smooth fibers scaffolds, whereas they showed a more spread morphology on the nano-porous fiber scaffolds. Shokrgozar et al. demonstrated that nanoroughness of PLGA electrospun scaffolds could enhance nerve cell growing rate up to $50 \%$ compared to smooth electrospun scaffolds [25]. Zhang et al. revealed that the introduction of elliptical nanopores onto the surface of aligned electrospun PLLA microfibers allowed for enhanced cellular response of vascular smooth muscle cells, including increased cell adhesion, proliferation, alignment, and expression of vascular matrix proteins [26]. However, all the above mentioned studies mainly focused on the influence of surface roughness of electrospun fibers on cellular adhesion and proliferation. Little knowledge on the effect of electrospun fiber surface nanoroughness on stem cell differentiation is available.

Here, we aimed at investigating the influence of electrospun fiber surface nanoroughness on the skeletal differentiation of hMSCs. The surface roughness of electrospun scaffolds was tailored through varying the humidity during electrospinning. The topographical surface was determined by scanning electron microscopy (SEM) and atomic force microscopy (AFM). The biological relevance of each surface roughness on osteogenic and chondrogenic cell differentiation was assessed in terms of cell morphology, metabolic activity, alkaline phosphatase (ALP) activity or glycosaminoglycan (GAG) production, and specific gene expression profiles.

\subsection{Materials and Methods}

\subsubsection{Fabrication of scaffolds}

Poly (ethylene oxide terephthalate)/poly(butylene terephthalate) (PEOT/PBT, the weight ratio of PEOT/PBT=55/45, the molecular weight $(\mathrm{g} / \mathrm{mol})$ of the starting $P E G$ segments used in the polymerization process is 300 ) was kindly provided by PolyVation B.V. (the Netherlands). A PEOT/PBT solution was prepared by dissolving the polymer in a mixture solvent of dichloromethane/ 1,1,1,3,3,3-Hexafluoro-2-propanol ( $/ \mathrm{v}=97 / 3)$ with a final concentration of $20 \%(\mathrm{w} / \mathrm{v})$. The electrospinning set-up was custom-made, consisted of an environmentally controlled electrospinning chamber connected to a syringe pump, and was able to move the spinneret in the chamber to obtain a homogeneous fiber distribution 
during the fabrication process. The polymer solution was fed at a rate of $8 \mathrm{~mL} / \mathrm{h}$ using a syringe pump (KDS 100, KD Scientific). The applied voltage and tip-to-collector distance were $20 \mathrm{kV}$ and $15 \mathrm{~cm}$, respectively. The temperature in the electrospinning chamber was monitored during the process and kept constantly around $20^{\circ} \mathrm{C}$. The relative humidity in the spinning chamber was chosen to be around $20 \%, 50 \%$ and $70 \%$, separately.

\subsubsection{Fiber morphology and dimensions}

The morphology of electrospun scaffolds was observed by SEM (Philips XL30 ESEMFEG). Prior to SEM imaging, samples were gold sputtered with a Cressington Sputter Coater 108 Auto set at $30 \mathrm{~mA}$ for 40 seconds. To create cross section images, samples were first frozen in liquid nitrogen and then cut. The average fiber diameter and the distribution of fiber diameters was calculated by measuring at least 30 fibers in one SEM image and five images were used for each scaffold using Adobe Photoshop CS4.

\subsubsection{Porosity and pore diameter of scaffolds}

Apparent density and porosity of scaffolds were calculated using the following equation [12]:

$$
p=\left(1-\frac{\rho^{\prime}}{\rho_{\circ}}\right) \times 100 \%
$$

Where $p$ is the porosity of scaffold porosity, $\rho^{\prime}$ is the apparent density of scaffold and $\rho^{\circ}$ the density of polymer materials used for fabricating scaffolds.

The pore diameter of scaffolds was calculated from SEM images using ImageJ according to previous study [29]. The diameters in each of at least 30 pores within an image for a total of four images per sample were quantified.

\subsubsection{AFM analysis}

Surface roughness analysis was performed through AFM using Tapping Mode (PicoScan Controller 2500, Molecular Imaging, USA) with a Super Sharp TESP cantilever: 42 N/m, $320 \mathrm{kHz}, 2-5 \mathrm{~nm}$ ROC, No Coatings (Bruker AFM Probes) on a $1 \mu \mathrm{m}^{2}$ surface area $(\mathrm{n}=6)$. The average roughness $(\mathrm{Ra})$, pore depth, and surface area differences were determined using the Scanning Probe Image Processor, SPIPTM, version 4.2.2.0 software. High quality images in 3D of the fiber surface were visualized at randomly different surface locations to verify the reproducibility of the observed characteristics. 


\subsubsection{Contact angle measurements}

The wettability of scaffolds was determined by the sessile drop technique using an optical contact angle device (OCA15, Dataphysics, Germany). The sessile milli-Q water drop was deposited onto the sample surface with the syringe, and the drop contour was fitted by the Young-Laplace method. At least 3 different static contact angle measurements on each sample were performed.

\subsubsection{Nile Red staining and analysis}

The local environment of polymer surfaces was determined using a Nile Red adsorption technique. Fabricated scaffolds or spin-cast polymer films (controls) were exposed to a dilute solution $(1 \mu \mathrm{M})$ of Nile Red in milli-Q water for 1 minute. After this minute, the samples were briefly washed with fresh milli-Q water to remove excess Nile Red solution; samples were then sandwiched between a glass slide and a coverslip. Images were taken with a confocal laser excitation microscope DM6000 (Leica, Wetzlar, Germany) equipped with DFC365FX camera. For measurement of the local environment, Nile Red $\left(\lambda_{\text {ex }}=514\right)$ emission was imaged over the range of $520-700 \mathrm{~nm}$ in $10 \mathrm{~nm}$ steps. Integrated intensity of the region of interest was then plotted against the average wavelength for each image, reconstituting the emission spectrum. All experiments were performed in duplicate, on two distinct samples (total of 4 measurements).

\subsubsection{Protein absorption on scaffolds}

Protein adsorption on electrospun scaffolds was investigated as previously described [21]. Briefly, samples were punched and placed in a 96 well plate. They were incubated in phosphate buffered saline (PBS) solution at $37{ }^{\circ} \mathrm{C}$ overnight. After removing PBS, $200 \mu \mathrm{L}$ of bovine serum albumin (BSA) solution (2 $\mathrm{mg} / \mathrm{mL}$ in PBS) was added to the wells and incubated at $37{ }^{\circ} \mathrm{C}$ overnight. The samples were rinsed with PBS to remove any nonadherent proteins. The amount of adsorbed protein was quantified using Bicinchoninic acid (BCA) protein assay kit (Thermo Scientific Pierce Protein Assay Kit). The absorbance was measured at $562 \mathrm{~nm}$ on a plate reader (Multiskan GO, Thermo Fisher, USA).

\subsubsection{Cell culture and seeding}

Human MSCs (donor No. 8001L) were isolated from bone marrow by Texas A\&M Health Science Center [30]. Briefly, bone marrow was aspirated centrifuged to separate mononuclear cells that would be considered as passage zero (PO) cells after being 
planted and harvested at $60-80 \%$ confluence. P0 cells were further expanded, harvested and frozen to obtain passage 1 (P1) cells. P1 cells were expanded in T-300 flasks at a density of $3 \times 10^{5}$ cells $/ \mathrm{cm}^{2}$ and cultured in basic medium (BM), comprising a-MEM (Gibco), 10\% fetal bovine serum (Lonza), $2 \mathrm{mM} \mathrm{L-glutamin} \mathrm{(Gibco),} 0.2 \mathrm{mM}$ ascorbic acid (Sigma), $100 \mathrm{U} / \mathrm{mL}$ penicillin and $100 \mathrm{mg} / \mathrm{ml}$ streptomycin (Gibco). Cells were harvested at approximately $80 \%$ confluence for further scaffold-based study.

Electrospun sheets were punched into discs $(1 \mathrm{~cm}$ in diameter) and placed in a 48-well plate. Rubber O-rings (Eriks B.V., The Netherlands) were put on the top of electrospun discs to prevent them from floating. For sterilization, the specimens were soaked in $70 \%$ $(\mathrm{v} / \mathrm{v})$ ethanol for $15 \mathrm{~min}$ three times. After that, they were rinsed with sterilized PBS $1 \mathrm{~min}$ for three times and immersed in BM overnight before cell seeding. After removing the medium from the scaffolds, hMSCs were seeded on each scaffold at a density of $1.5 \times 10^{4}$ cells $/ \mathrm{cm}^{2}$ in $80 \mu \mathrm{L} \mathrm{BM}$. Cell-scaffold constructs were incubated for 4 hours to allow cell attachment, and topped up to $1 \mathrm{~mL}$ of $\mathrm{BM}$, osteogenic medium (OM) (BM plus $10^{-6} \mathrm{M}$ dexamethasone), or chondrogenic medium (CM). CM was composed of DMEM (Gibco), 1\% ITS premix (Micronic BV), $50 \mu \mathrm{g} / \mathrm{mL}$ ascorbic acid (Sigma-Aldrich), 100 units $/ \mathrm{mL}$ penicillin (Life technologies), $100 \mu \mathrm{g} / \mathrm{mL}$ streptomycin (Life technologies), $100 \mathrm{nM}$ dexamethasone (Sigma-Aldrich), $40 \mu \mathrm{g} / \mathrm{mL}$ proline (Sigma-Aldrich), $100 \mu \mathrm{g} / \mathrm{mL}$ sodium pyruvate (Life technologies), $0.01 \mu \mathrm{g} / \mathrm{mL}$ TGF- $\beta 3$ (R\&D systems). The cell-scaffold constructs were cultured in an incubator with $5 \% \mathrm{CO}_{2}$ humid atmosphere at $37^{\circ} \mathrm{C}$ up to 21 days. Culture medium was refreshed every two days.

\subsubsection{Metabolic activity}

PrestoBlue $^{\mathrm{TM}}$ assay was used to assess cellular metabolism. Briefly, cell culture medium was replaced with a medium containing $10 \% \quad(\mathrm{v} / \mathrm{v})$ PrestoBlue ${ }^{\mathrm{TM}}$ reagent (Life technologies) and the plates were wrapped with aluminum foil to avoid light, followed by incubating at $37^{\circ} \mathrm{C}$ for $1 \mathrm{~h}$. Fluorescence was measured at $590 \mathrm{~nm}$ with a Perkin Elmer Victor3 1420 multilabel plate reader. Cell metabolic activity was analyzed on day 7 and day 21.

\subsubsection{Quantification of DNA}

The total amount of DNA per scaffold was quantified as described in a previous study [31]. Briefly, samples were washed twice with PBS and then processed to digest for $16 \mathrm{~h}$ in a Tris/EDTA buffer composed of $1 \mathrm{mg} / \mathrm{mL}$ proteinase $\mathrm{K}, 18.5 \mu \mathrm{g} / \mathrm{mL}$ iodoacetamine (SigmaAldrich) and $1 \mu \mathrm{g} / \mathrm{mL}$ Pepstatin A (Sigma-Aldrich). The lysate was divided into two parts. 
One part was used for ALP or GAG assay and the others were used for DNA assay. For DNA quantification, the lysate was added to a buffer containing RNase $(\times 1000)$, component $\mathrm{B}$ (part of the DNA quantification kit) and NaCl-EDTA buffer. Subsequently, quantification of total amount of DNA per scaffold was performed using the CyQuant ${ }^{\circledR}$ DNA assay (Life Technologies) according to manufacturer's protocol. The signal was measured on a spectrophotometer (Victor3 1420, Perkin Elmer, USA) at an excitation wavelength of $480 \mathrm{~nm}$ and emission wavelength of $520 \mathrm{~nm}$.

\subsubsection{Alkaline phosphatase (ALP) activity assay}

The ALP activity was assessed as in a previous study [21]. Briefly, Cell lysate mentioned in DNA assay paragraph were digested for $1 \mathrm{~h}$ at room temperature with a buffer at a $\mathrm{pH}$ of 7.8 composed of $0.1 \mathrm{M} \mathrm{KH}_{2} \mathrm{PO}_{4}, 0.1 \mathrm{M} \mathrm{K}_{2} \mathrm{HPO}_{4}$ and $0.1 \%$ Triton $\mathrm{X}-100$. After that, a CDP-star ${ }^{\circledR}$ solution (Roche Life Science) was added and samples were incubated for 15 min at room temperature. The signal was analyzed using a Victor3 1420 plate reader (Perkin Elmer, USA). The value of ALP activity was normalized to DNA quantity per scaffold.

\subsubsection{Glycosaminoglycan (GAG) assay}

Cell lysates mentioned in DNA assay paragraph were pipetted in duplicate to 96-well plates. After addition of a dimethylmethylene blue dye (DMMB, Sigma-Aldrich) solution comprising $10 \mathrm{mM}$ hydrochloric acid, $3.04 \mathrm{~g} / \mathrm{L}$ of glycine and $2.37 \mathrm{~g} / \mathrm{L}$ of $\mathrm{NaCl}$, the plates were measured at an absorbance of $525 \mathrm{~nm}$ using a micro plate reader (Multiskan GO, Thermo Fisher, USA). The amount of GAG was calculated using a standard of chondroitin sulfate (Sigma-Aldrich).

\subsubsection{RNA isolation and gene expression}

Total RNA per scaffold was isolated following the protocol based on a previous study [32]. Briefly, samples were rinsed with PBS three times prior to adding $1 \mathrm{~mL}$ TRIzol reagent (Invitrogen). Subsequently, $200 \mu \mathrm{L}$ of chloroform was added to all samples, mixed vigorously, and centrifuged at $1200 \mathrm{~g}$ for $15 \mathrm{~min}$ at $4{ }^{\circ} \mathrm{C}$. The aqueous phase containing RNA was transferred to filter columns provided by the NucleoSpinRNAll ISOLATON kit (Bioke) and the purification process was performed according to the manufacture's protocol. The concentration and purity of total RNA were determined by using an ND 1000 Nanodrop (Life Technologies, USA). One $\mu \mathrm{g}$ of total RNA was used for first strand cDNA synthesis using iScript (Bio-Rad, Veenendaal, the Netherlands) following the 
manufactures protocol. Quantitative polymerase chain reaction (qPCR) was carried out on a BioRad CFX96 real time PCR machine using SYBR-green supermix (Bio-Rad) and the primers as listed in Table 1. The Cycling conditions for running qPCR were as following: initial denaturation at $95^{\circ} \mathrm{C}$ for $10 \mathrm{~min}$, followed by 40 cycles of $15 \mathrm{~s}$ at $95{ }^{\circ} \mathrm{C}$ and $1 \mathrm{~min}$ at $60^{\circ} \mathrm{C}$. Relative expression of RNA was normalized to housekeeping gene $\mathrm{B} 2 \mathrm{M}$, and fold changes were calculated using the $2^{-\triangle \Delta C T}$ method, where

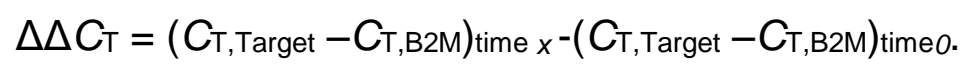

\subsubsection{Statistical analysis}

Statistical analysis was determined using GraphPad Prism 5.01 (GraphPad software, San Diego, USA) for windows. Two-way analysis of variance (ANOVA), followed by a Tukey's post-hoc test was performed to compare the gene expression, metabolic activity, GAG production and ALP activity. One-way ANOVA with Bonferroni's multiple comparison tests was used to compare the surface roughness. A $p$ value lower than 0.05 was considered statistically significant.

\subsection{Results and Discussion}

\subsubsection{Characterization of electrospun scaffolds}

The relative humidity showed a remarkable effect on the surface roughness of electrospun scaffolds. At a relative humidity of $20 \%$, the morphology of fibers was smooth (Figure $1 \mathrm{~A}$ and 1D). Increasing the relative humidity to $50 \%$ resulted to apparent changes in surface morphology of fibers. Figure $1 \mathrm{E}$ shows that surface features or pores become evident. When the humidity increased from $50 \%$ to $70 \%$, the pore depth increased as well as the pore size leaving little space between adjacent pores. The formation mechanism of pores on electrospun fibers could be elucidated using the combination of vapor-induced phase separation with thermal-induced phase separation [9, 16]. In a vapor-induced phase separation model, the non-solvent (water) from vapor phase penetrates into the polymer solution causing phase separation into a solvent-rich phase and solid-rich phase. On the other hand, in the thermal- induced phase separation model, the volatile solvent evaporates dramatically, the liquid jet cools down, leading to a phase separation into a polymer-rich phase and solvent-rich phase. In both models, the solvent-rich phase will cause formation of pores on the fiber surface, whereas the polymer-rich phase will eventually evolve into a solid matrix. The cross-section images (Figure 1G-I) indicated that all fibers had a solid interiors wrapped by an increasingly porous outer layer with increasing humidity. 


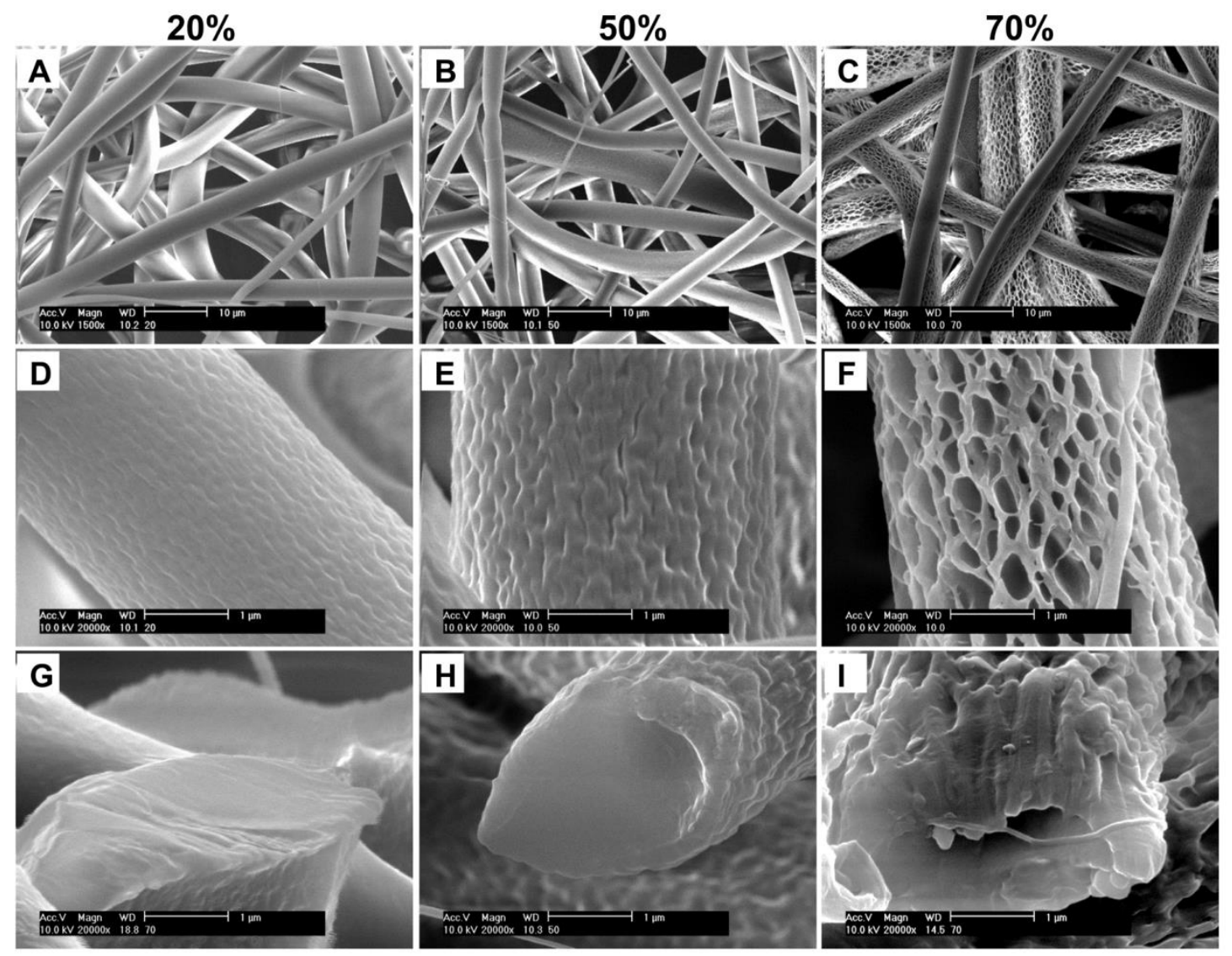

Figure1. SEM image of PEOT/PBT fibers $(A-C)$ and their corresponding surfaces (D-F) and cross sections (G-I) fabricated by electrospnining under different relative humidity: $20 \%(A, D, G), 50 \%(B, E, H)$ and $70 \%$ $(C, F, I)$. Scale bars are $10 \mu \mathrm{m}(A-C)$ and $1 \mu \mathrm{m}(\mathrm{D}-\mathrm{I})$.

In addition, to investigate the effect of humidity on the topology of pores, AFM analysis was performed (Figure 2A). Three dimensional representations of surface topography for the electrospun fibers illustrate the differences in surface roughness between fibers by varying the humidity. These results are in agreement with the SEM data. The quantification results of surface roughness parameters including $\mathrm{Ra}$, pore depth and surface area difference are presented in Figure 2B. A significant difference in surface roughness was observed between fibers fabricated at different humidity. Changing humidity from $20 \%$ to $70 \%$ corresponded to increasing Ra from $14.3 \pm 2,5 \mathrm{~nm}$ to $71,0 \pm$ $11.0 \mathrm{~nm}$, surface area from $3.1 \pm 0.8 \mathrm{~nm}$ to $63.3 \pm 6.2 \mathrm{~nm}$ and pores depth from $27.2 \pm 4.7$ $\mathrm{nm}$ to $230.5 \pm 31.9 \mathrm{~nm}$. Altogether, increasing of relative humidity resulted in an increase of roughness, depth, and surface area of pores on the surface of electropun fiber. Similar findings were also reported by previous studies where a more porous morphology appeared on the surface of polystyrene fibers as electrospinning was performed in a higher-humidity environment [16, 33, 34]. 

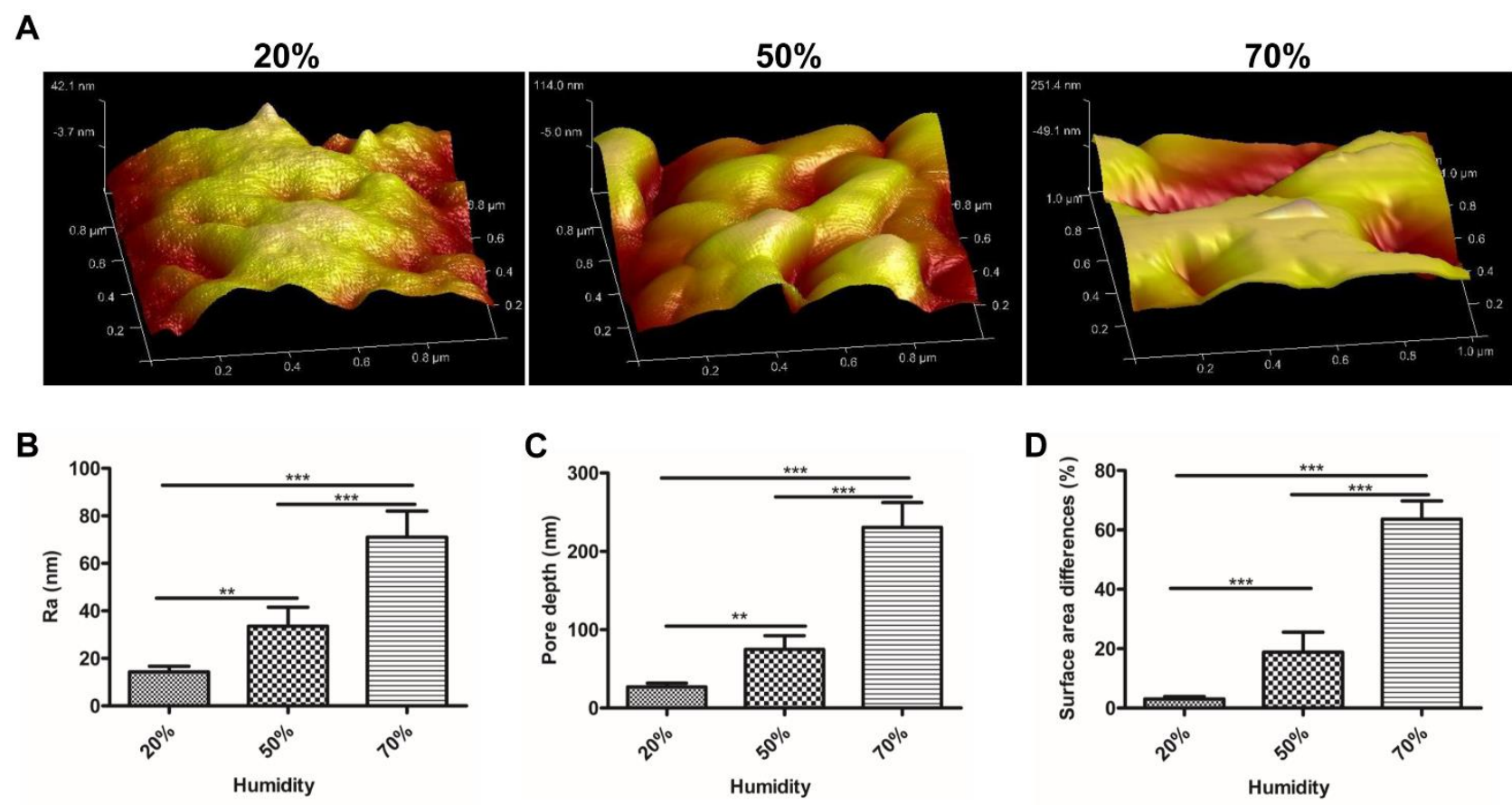

Figure 2. (A) AFM images of $20 \%$ sample, $50 \%$ sample, and $70 \%$ sample. Histograms of (B) average surface roughness $(\mathrm{Ra}),(\mathrm{C})$ pore depth and $(\mathrm{D})$ surface area differences for obtained electrospun fibers. ${ }^{* *} p<0.01,{ }^{* * *} p<0.001$

Previous studies have demonstrated that fiber diameter, pore size and porosity of scaffolds influence cell behavior such as proliferation, attachment and differentiation [3537]. To better understand the effect of nanosurface roughness on hMSCs differentiation, the surface roughness was varied while keeping other structural parameters, such as fiber diameter, pore size and porosity of the scaffolds constant. As shown in Figure $3 \mathrm{~A}$ and $B$, no significant difference in porosity and pore size of the electrospun scaffolds was found when changing the relative humidity. In addition, the obtained electrospun scaffolds had average fiber diameters of $3.8 \pm 0.8,3.5 \pm 1.0$, and $4.0 \pm 1.2 \mu \mathrm{m}$ for spinning from relative humidity of $20 \%, 50 \%$ and $70 \%$, respectively (Figure $3 \mathrm{C}-\mathrm{E}$ ). The relative humidity is known to have an impact on the fiber dimension which is essentially dependent on the polymer concentration as well as solvent used [34]. Given the fiber dimension scale range achieved in the present study, increasing relative humidity did not cause apparent difference in fiber dimension. 

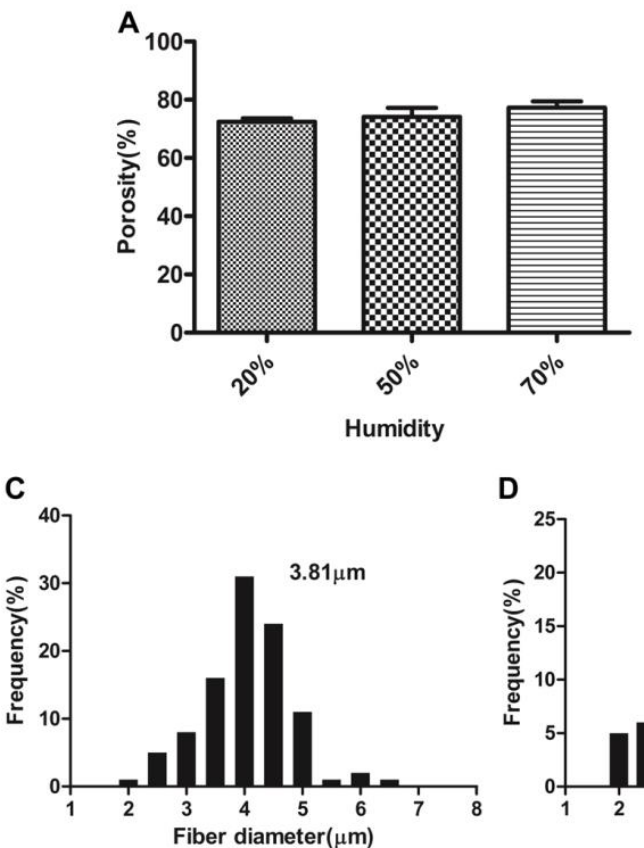
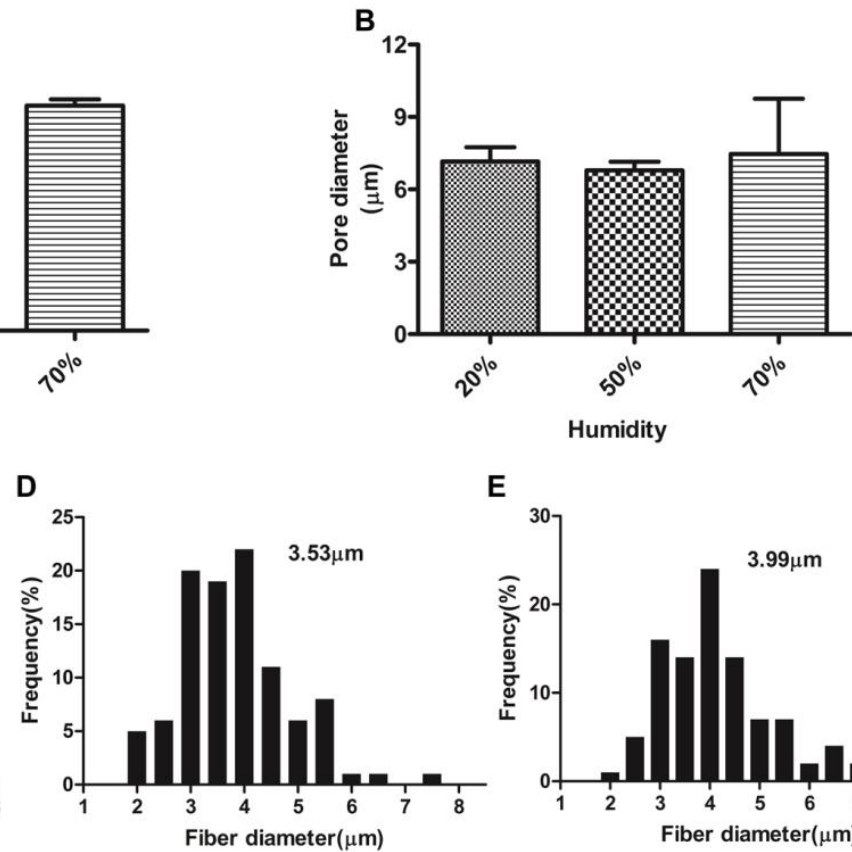

E

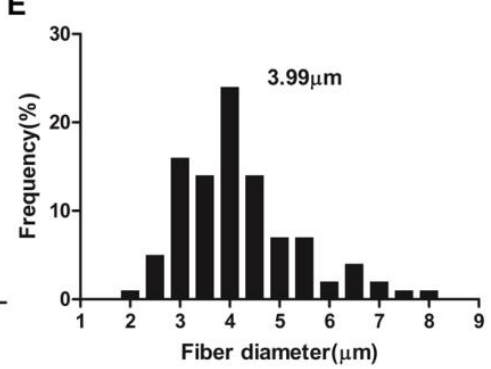

Figure 3. The porosity (A) and pore diameter (B) of scaffolds indicating no significant differences. Distribution of fiber diameter for $20 \%$ samples (C), $50 \%$ samples (D) and $70 \%$ samples (E).

Chemical functional groups and/or blocks of the polymer chains may be reoriented during electrospinning process [38]. To determine the influence of relative humidity on the surface polarity of electrospun fiber, a Nile Red assay was employed (Figure 4). Nile Red is a hydrophobic and solvatochromic dye [39] that has previously been used to determine the hydrophilicity/phobicity of a wide range of polymers in the bulk [40] and the variability of polymer surface environments [41]. Shifts in the absorption or emission maximum of the dye adsorbed to a polymer indicate differences in hydrophilicity/phobicity (dielectric strength). The electrospun fibers were stained with Nile Red homogeneously (Figure S1). As shown in Figure 4, for all electrospun scaffolds (20\%, 50\%, and $70 \%$ ), we found nearly identical emission intensity profiles (emission $\lambda_{\max }=600 \mathrm{~nm}$ ); this is in contrast to control samples, where we observed shifts in the emission intensity based on polymer composition (see SI for details). The identical emission profile of Nile Red across all our samples suggests that the electrospun scaffolds have similar polarity on their surface. To confirm these measurements, control samples of spin-cast poly (methyl methacrylate) (PMMA) and polycaprolactone ( $P C L)$ were shown to have distinctly different Nile Red emission profiles. The PMMA had a lower emission profiles compared to PCL (Figure S2), which support the fact that PMMA was more hydrophobic than PCL [40]. 


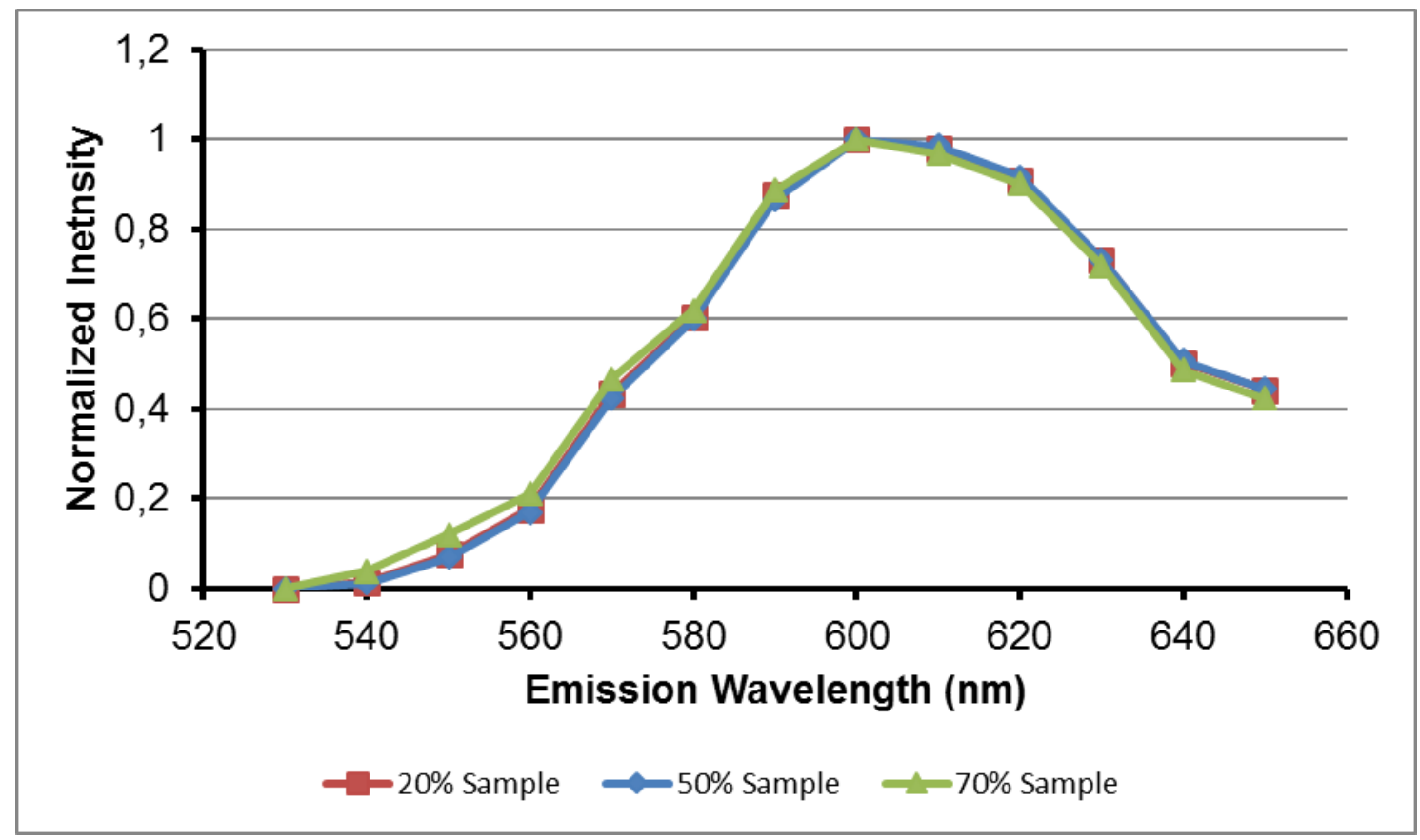

Figure 4. Nile Red emission profile of electrospun samples. Note that the samples have overlapping emission intensity profiles. $\lambda_{\mathrm{ex}}=514$.

Surface wettability of scaffolds is important for initial cell attachment and migration [42]. Wettability was assessed using contact angle measurements (Figure 5A and B). Generally, water sessile droplets penetrated into all scaffolds in the end, indicating hydrophilic properties of the mesh surface, probably due to the same chemical composition of scaffolds. In the present study, the material used for scaffold fabrication was PEOT/PBT which had a medium surface energy with an average contact angle of $48 \pm 1^{\circ}$ [43]. Although water droplets would ultimately penetrate into all scaffolds, their spreading speed was different showing an inverse relationship with the surface roughness of scaffolds. Surface roughness, fiber diameter, and pore size are important factors affecting the contact angle of a surface, where the material itself is not varied [44]. As shown above, the scaffolds fabricated in different relative humidities displayed similar fiber dimension, pore size and surface chemistry, but varied surface roughness. Therefore, in the present study, the differences in surface energy were mainly attributed to the surface roughness. These findings are consistent with the results reported by Chuah et al., who demonstrated that increasing the surface roughness of polydimethylsiloxane (PDMS) substrates could enhance the hydrophobicity [45]. 
A

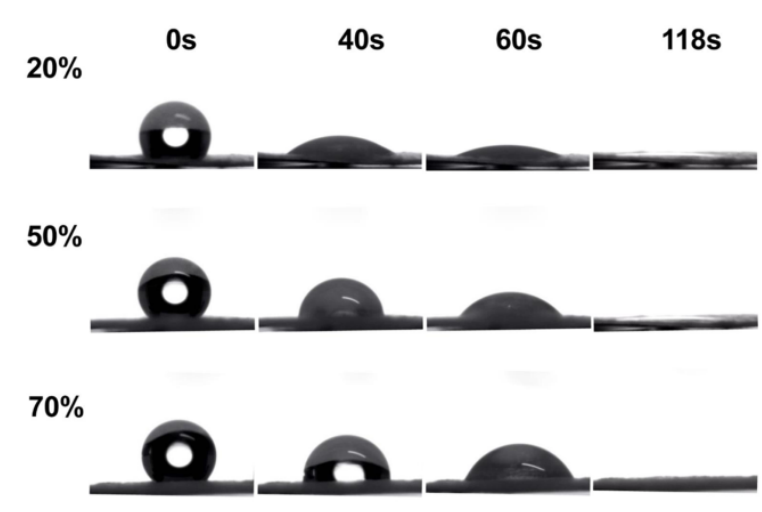

B

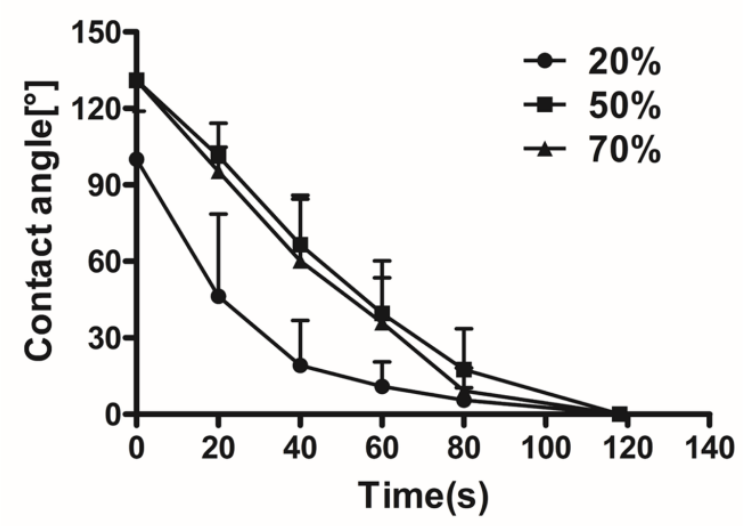

Figure 5. Contact angle measurement of electrospun scaffolds with different surface roughneess. (A) representative images showing droplets on electrospun scaffolds surface roughneess; (B) time-dependency of contact angle on scaffolds.

\subsubsection{Protein adsorption}

Protein adsorption on the different scaffolds was examined using BSA as a model protein, and the results are shown after normalizing to scaffolds fabricated under a relative humidity of $20 \%$. As shown in Figure 6, no significantly difference in protein adsorption was observed between scaffolds from a relative humidity of $20 \%$ and $50 \%$, which may be attributed to their closed surface structure. On the other hand, scaffolds from a relative humidity of $70 \%$ showed significant higher protein adsorption than that of scaffolds from a relative humidity of $20 \%$.This could be related to the increased surface roughness and surface porosity leading to a higher surface area. Such an increased protein absorption was also shown by Nandakumar et al.[21] who used gas plasma for surface modification of electrospun fibers. A higher protein adsorption on scaffolds is also known to influence the cellular response such as cell adhesion, proliferation and differentiation [46, 47]. 


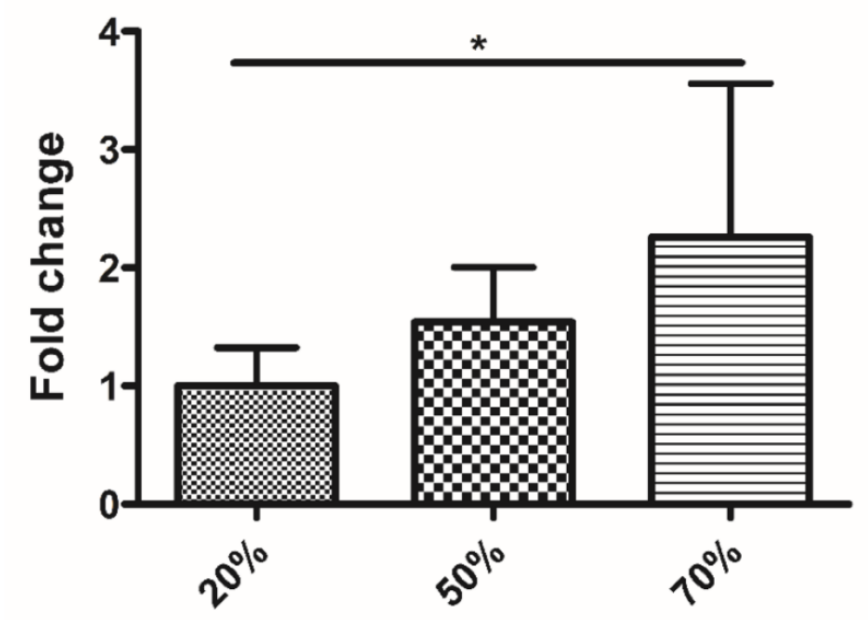

Figure 6. Adsorption of BSA on electrospun scaffolds with different surfaces.

\subsubsection{Metabolic activity of cells}

Metabolic activity of hMSCs seeded on scaffolds characterized by different surface roughness was investigated by prestoblue ${ }^{\mathrm{TM}}$ assay (Figure 7 ). The metabolic activity rate in BM at day 7 was similar to that of day 21 , while a slightly lower metabolic activity was measured in $\mathrm{OM}$ and $\mathrm{CM}$ after 21 days. The decrease in metabolic activity rate in OM and CM could be ascribed to the differentiation of hMSCs No statistical differences in metabolic activity were observed between the different surfaces roughnesses of the scaffolds in all media investigated in this study.

A

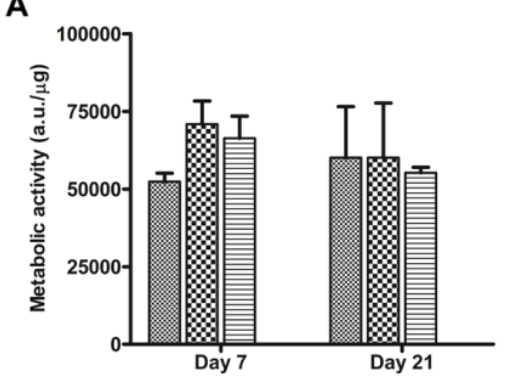

B

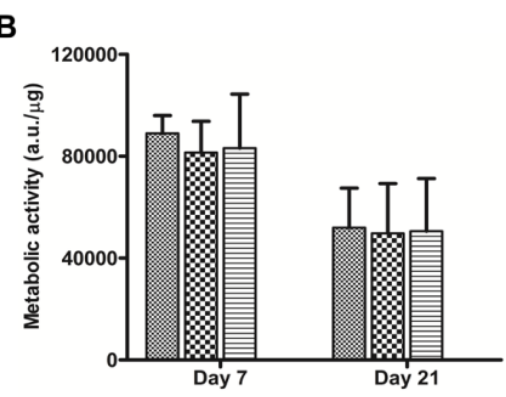

C

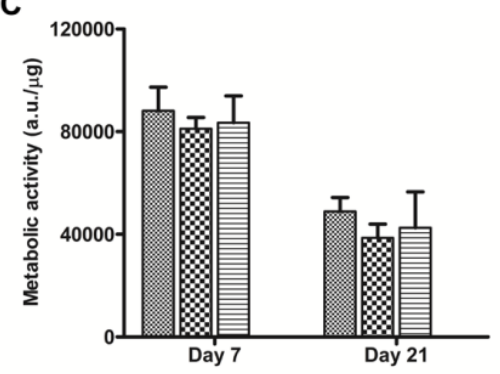

ख $20 \%$

D0\% $50 \%$

Figure 7. Metabolic activity of hMSCs cultured on scaffolds in BM (A), OM (B) and CM (C) at day 7 and day 21. 


\subsubsection{ALP activity and GAG production}

ALP activity, a common marker for early osteogenesis, is known to be up-regulated in the early stages of osteogenic differentiation [48]. As shown in Figure 8A-B and Figure S3, at day 7 , a similar ALP activity in all kind of scaffolds was found in both BM and OM. In contrast, fibers (the $70 \%$ sample) with a surface roughness of $71.0 \pm 11.0 \mathrm{~nm}$ displayed a higher ALP activity compared to fibers (the $20 \%$ sample) with a roughness of $14.3 \pm 2.8$ $\mathrm{nm}$ in BM at day 21. The similar trend was also observed in OM. Therefore, it could be inferred that a higher surface roughness causes a higher ALP activity in the range studied in the present work. Our results are consistent with previous studies [49, 50], where osteoblasts were demonstrated to have a higher ALP activity on a rougher surface of titanium substrates. Furthermore, our previous study has also demonstrated that hMSCs express evidently higher ALP activity on ceramic nanofibers displaying a rougher surface $(0.7 \pm 0.2 \mathrm{~nm})$ compared to a smoother one $(0.4 \pm 0.1 \mathrm{~nm})$ under osteogenic culture condition [51].

GAG is a long-chain sugar molecule and plays an essential role in chondrogenic differentiation, since it is one of the main components of the proteoglycans forming articular cartilage extracellular matrix [52]. In a previous study by our group, additive manufactured three-dimensional (3D) scaffolds whose polymer fibers have tailored surface roughness (ranging from $0.07 \pm 0.01 \mu \mathrm{m}$ to $1.95 \pm 0.55 \mu \mathrm{m}$ ) were fabricated in combination with a wet-spinning technique [53]. The results demonstrated that hMSCs on a highest roughness scaffolds $(1.95 \pm 0.55 \mu \mathrm{m})$ showed a higher GAG production under chondrogenic culture condition. Despite the GAG production in all types of scaffolds enhanced over the experimental time course in all media (Figure 8C-D and Figure S4), no statistical difference in GAG production was found between the different surface roughnesses, indicating that the surface roughness nanometric range investigated in this study does not significantly influence GAG production by hMSCs during differentiation into the chondrogenic lineage. 
A

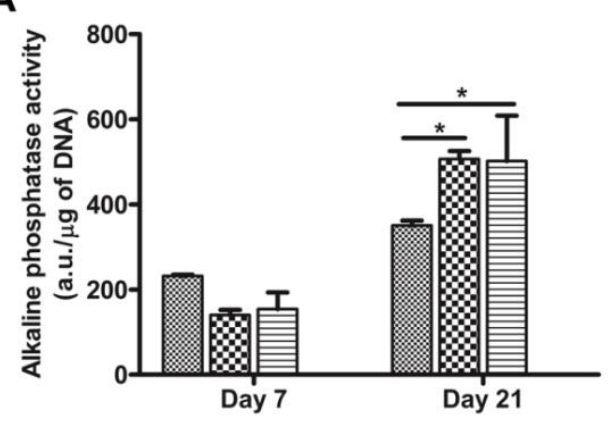

C

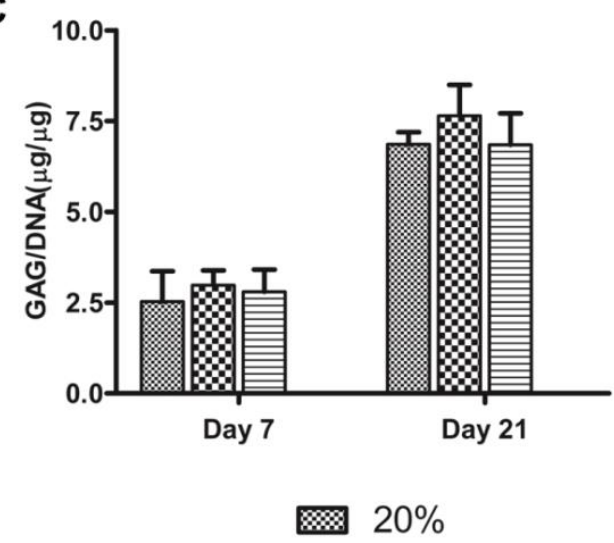

B

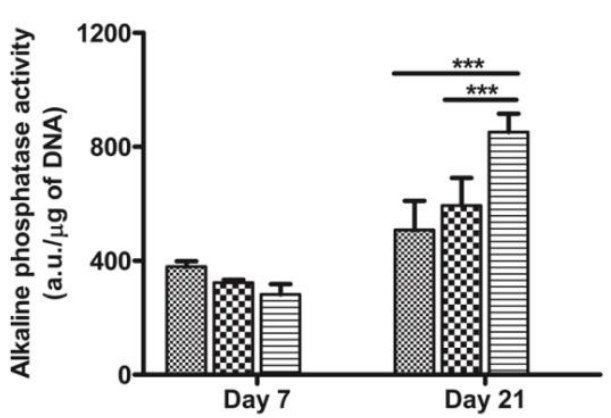

D

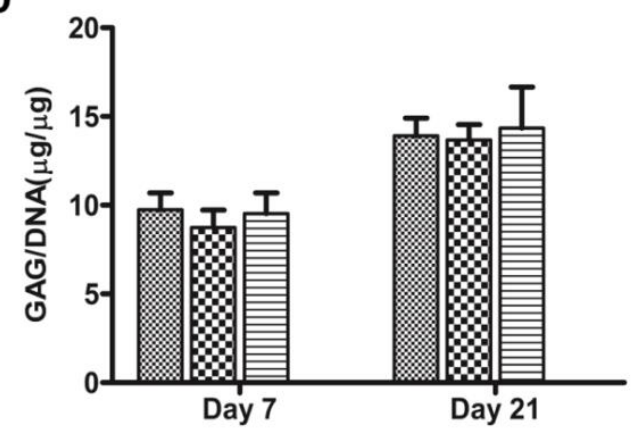

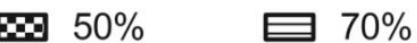

Figure 8. ALP activity of hMSCs cultured on scaffolds in BM (A) and OM (B) at days 7 and 21. GAG production on scaffolds in BM (C) and CM (D) at day 7 and day 21. The final results of ALP activity and GAG production level was normalized by DNA amount. ${ }^{*} p<0.05,{ }^{* * *} p<0.001$.

\subsubsection{Osteogenic gene expression}

The influence of surface roughness on osteogenic differentiation was confirmed by real time RT-PCR (Figure 9). Osteopontin (OPN) is known as a major noncollagenous extracellular structural protein with respect to cell adhesion, migration, and biomineralization [54]. Bone morphogenetic protein-2 (BMP2) is known to induce osteogenesis of hMSCs [55]. At day 7 in BM, OPN and BMP2 expressions were significantly higher for rougher fibers (the $70 \%$ sample) compared to smoother fibers (the $20 \%$ sample). One the other hand, bone sialoprotein (BSP), collagen type I (COL1A1) and osteocalcin $(\mathrm{OCN})$, which are extracellular matrix proteins of bone [56], showed higher expression on smootherfibers (the $20 \%$ sample) compared to rougher fibers(the $70 \%$ sample). To know the osteoconductive properties of different surface roughness, the same panel of osteogenic markers was also investigated in OM. In the investigated surface roughness range, RUNX2 expression was significantly higher on rougher fibers (the $70 \%$ sample) compared to smoother fibers (the $20 \%$-sample) at day 7 . Conversely, BSP expression on smoother fibers (the $20 \%$ sample) was significantly higher than that 
on rougher fibers (the $70 \%$ sample) after day 21 . Recent studies revealed that the hierarchical combination of micro- and nanoscale surface features promotes osteogenic differentiation [57, 58]. Khang. et al. reported that a stronger osteogenic differentiation of hMSCs was supported by a heterogeneous composition of nano and sub-micron hybrid surface features compared to only nano scale or sub nano scale features [57]. In another study, Gittens et al. demonstrated that a combination of micro-/submicro-scale surface roughness with nanoscale structures on Titanium surfaces enhanced osteoblast differentiation [58]. Therefore, it could be assumed that the hierarchical combination of different surface nano-roughness in electrospun fibers may be more beneficial on the osteogenic differentiation of hMSCs.

\subsubsection{Chondrogenic gene expression}

A panel of chondrocyte-related genes, including collagen type II alpha 1 (COL2A1), activated leukocyte cell adhesion molecule (ALCAM), SOX9, and aggrecan (ACAN) was investigated to assess hMSCs chondrogenesis (Figure 10). In BM, only SOX9 was differentially regulated. On the other hand, the gene expression profile was much different under chondrogenic differentiation culture conditions, most likely due to the dominant effect of chemical stimuli. In CM, upregulation of all tested chondrogenic markers was observed on the $20 \%$ sample compared to the $70 \%$ sample due to their different surface roughness. However, this trend was disappeared after 21 days. Recent work suggested that the tendency of hMSCs differentiation which is trigged by surface topography stimuli may be discontinued when the surface topography cues disappear [53, 59]. This might have also happened in this study when hMSCs were fully confluent on scaffolds and started being embedded within the endogenous ECM. SOX9 is known as an early chondrogenic transcription factor which control the expression of genes related to matrix secretion [60]. Upregulation of SOX9 could be activated in mouse stromal ST2 cells cultured in low oxygen tension (hypoxic environment) [61]. The expression of SOX9 was significantly higher on rougher fibers (the $70 \%$ sample) than on smoother fibers (the $20 \%$ sample) in both $\mathrm{BM}$ and $\mathrm{CM}$ after 21 days. This might be related to the higher spatial proximity of the hMSCs cultured in rougher scaffolds, which facilitate cells condensation resulting in hypoxic condition when cells are confluent on the scaffolds [53, 62]. 
BM
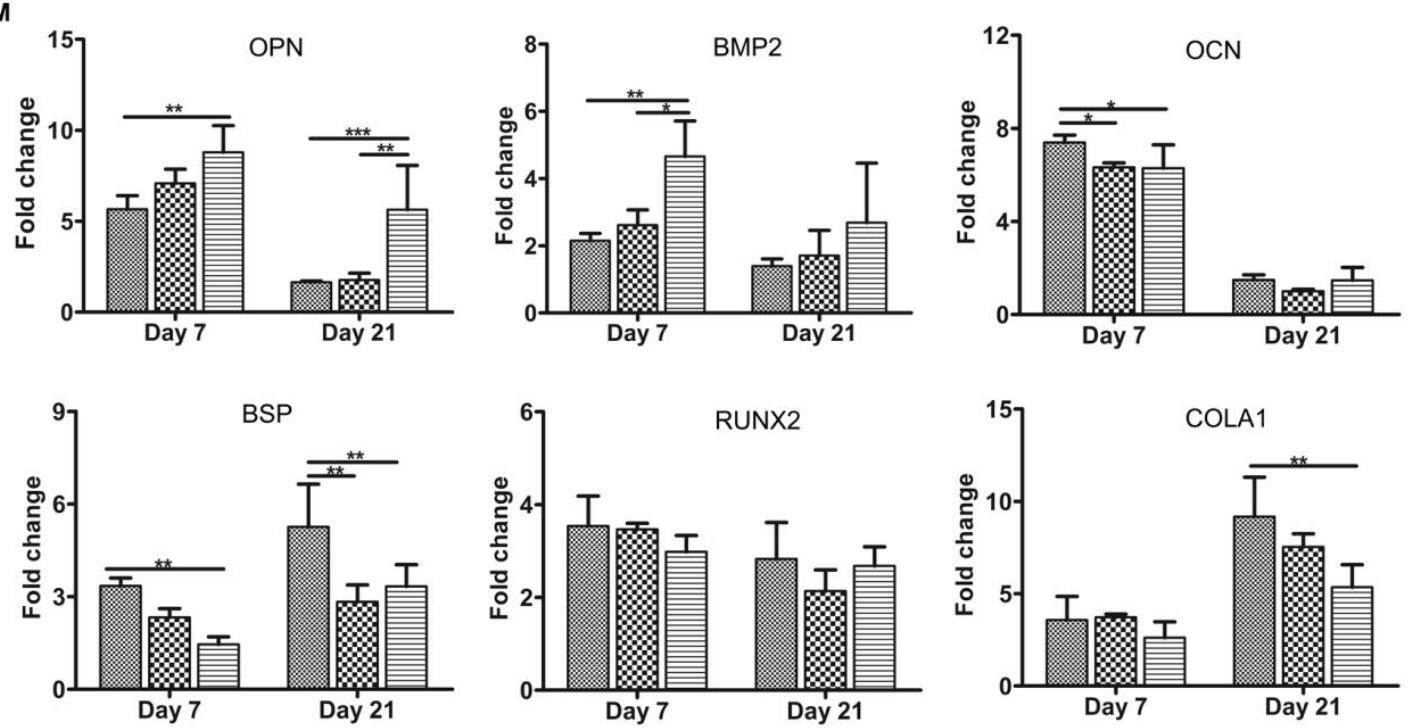

OM
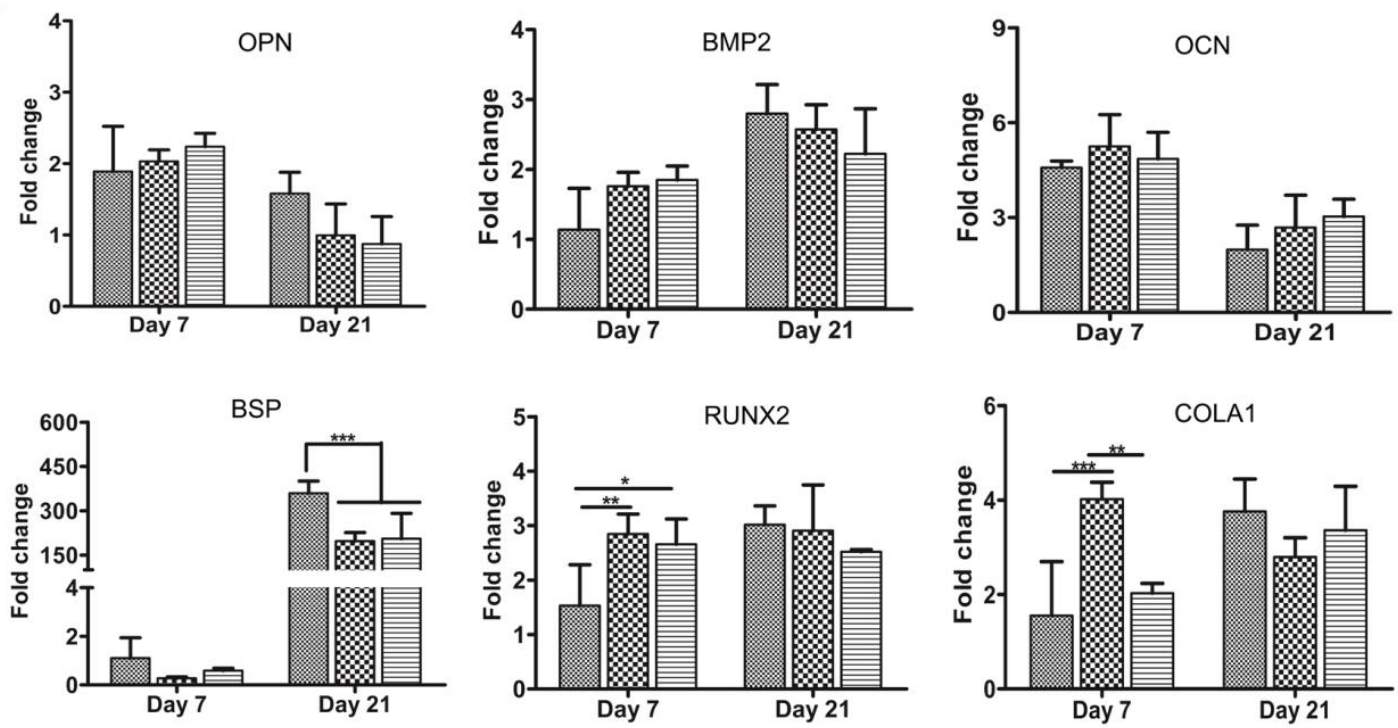

æ20\%

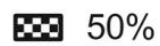

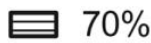

Figure 9. Quantitative RT-PCR gene expression analysis of osteoblast-related genes for hMSCs cultured on scaffolds in both BM and OM at days 7 and $21 .{ }^{*} \mathrm{p}<0.05,{ }^{* *} \mathrm{p}<0.01,{ }^{* * *} \mathrm{p}<0.001$. 
SOX9

COL2A1

ALCAM

ACAN

BM
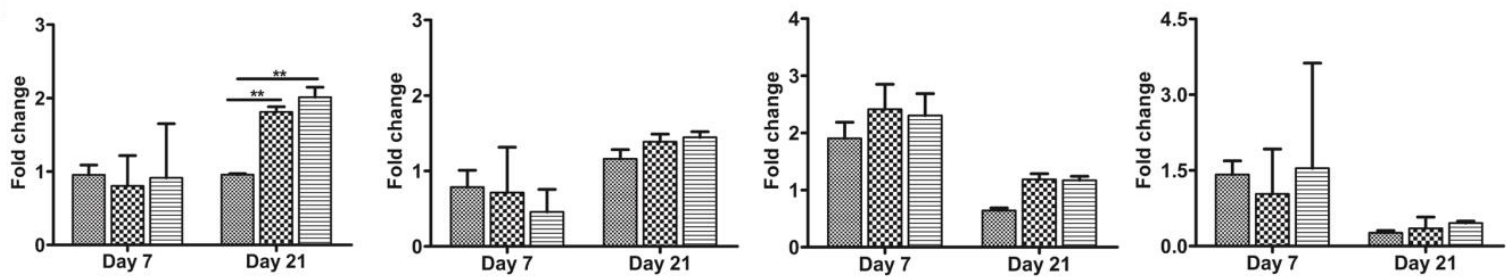

$\mathrm{CM}$
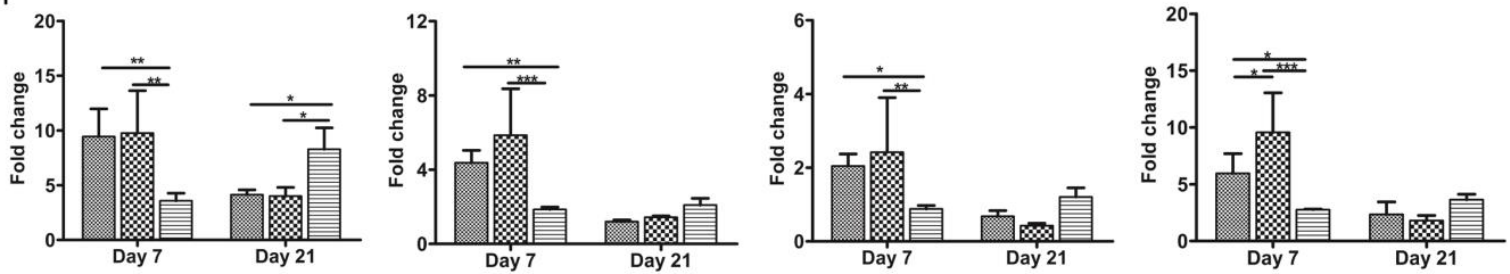

$20 \%$

50\%

$70 \%$

Figure 10. Quantitative RT-PCR gene expression analysis of chondrocyte-related genes for hMSCs cultured on scaffolds in $\mathrm{BM}$ and $\mathrm{CM}$ at time points of day 7 and day $21 .{ }^{*} \mathrm{p}<0.05,{ }^{* *} \mathrm{p}<0.01,{ }^{* * *} \mathrm{p}<0.001$.

\subsection{Conclusion}

In the present work, three types of electrospun fibers with surface nanotopography were directly fabricated by varying the relative humidity during electrospinning. These scaffolds had similar fiber dimensions, porosity and pore size, and chemical composition of the surface, yet a different surface roughness on the fibers. SEM and AFM results demonstrated that higher relative humidity resulted in higher surface roughness, pore depth and surface area in the nanoscale range. In order to investigate the impact of individual fiber surface nanotopography on cell differentiation, hMSCs were cultured on scaffolds for 21 days in osteogenic and chondrogenic differentiation media. We found that a higher surface roughness $(\mathrm{Ra}=71.0 \mathrm{~nm}$ ) showed enhanced expression of osteogenic genes such as OPN, BMP2, and RUNX2, while a lower surface roughness $(R a=14.3 \mathrm{~nm})$ demonstrated enhanced other osteogenic genes expression, including BSP, COL1A1 and OCN. Interestingly, a lower surface roughness $(\mathrm{Ra}=14.3 \mathrm{~nm})$ showed to better support the chondrogenic differentiation of hMSCs at day 7 compared to higher surface roughness $(\mathrm{Ra}=71.0 \mathrm{~nm})$. Tailoring surface nanoroughness of electrsopun scaffolds could, therefore, be used as a promising approach to control the cellular response of hMSCs in tissue engineering and regenerative medicine. 


\section{Reference}

[1] Y.R.V. Shih, C.N. Chen, S.W. Tsai, Y.J. Wang, O.K. Lee, Growth of mesenchymal stem cells on electrospun type I collagen nanofibers, Stem Cells 24(11) (2006) 2391-2397.

[2] M.B. Mueller, R.S. Tuan, Functional characterization of hypertrophy in chondrogenesis of human mesenchymal stem cells, Arthritis \& Rheumatism 58(5) (2008) 1377-1388.

[3] K. Le Blanc, M. Pittenger, Mesenchymal stem cells: progress toward promise, Cytotherapy 7(1) (2005) 36-45.

[4] M.F. Pittenger, A.M. Mackay, S.C. Beck, R.K. Jaiswal, R. Douglas, J.D. Mosca, M.A. Moorman, D.W. Simonetti, S. Craig, D.R. Marshak, Multilineage potential of adult human mesenchymal stem cells, science 284(5411) (1999) 143-147.

[5] K. Pelttari, E. Steck, W. Richter, The use of mesenchymal stem cells for chondrogenesis, Injury 39(1) (2008) 58-65.

[6] A.B. Faia-Torres, S. Guimond-Lischer, M. Rottmar, M. Charnley, T. Goren, K. Maniura-Weber, N.D. Spencer, R.L. Reis, M. Textor, N.M. Neves, Differential regulation of osteogenic differentiation of stem cells on surface roughness gradients, Biomaterials 35(33) (2014) 9023-9032.

[7] C.J. Bettinger, R. Langer, J.T. Borenstein, Engineering substrate topography at the micro-and nanoscale to control cell function, Angewandte Chemie International Edition 48(30) (2009) 5406-5415.

[8] M.J. Dalby, N. Gadegaard, R. Tare, A. Andar, M.O. Riehle, P. Herzyk, C.D. Wilkinson, R.O. Oreffo, The control of human mesenchymal cell differentiation using nanoscale symmetry and disorder, Nature materials 6(12) (2007) 997-1003.

[9] C.L. Casper, J.S. Stephens, N.G. Tassi, D.B. Chase, J.F. Rabolt, Controlling surface morphology of electrospun polystyrene fibers: effect of humidity and molecular weight in the electrospinning process, Macromolecules 37(2) (2004) 573-578.

[10] D. Li, Y. Xia, Electrospinning of nanofibers: reinventing the wheel?, Advanced materials 16(14) (2004) 1151-1170.

[11] H. Chen, X. Fan, J. Xia, P. Chen, X. Zhou, J. Huang, J. Yu, P. Gu, Electrospun chitosan-graft-poly ( $\varepsilon-$ caprolactone)/poly ( $\varepsilon$-caprolactone) nanofibrous scaffolds for retinal tissue engineering, International journal of nanomedicine 6 (2011) 453.

[12] H. Chen, J. Huang, J. Yu, S. Liu, P. Gu, Electrospun chitosan-graft-poly ( $\varepsilon$-caprolactone)/poly ( $\varepsilon$ caprolactone) cationic nanofibrous mats as potential scaffolds for skin tissue engineering, International journal of biological macromolecules 48(1) (2011) 13-19.

[13] A. Nandakumar, R. Truckenmüller, M. Ahmed, F. Damanik, D.R. Santos, N. Auffermann, J. de Boer, P. Habibovic, C. van Blitterswijk, L. Moroni, A fast process for imprinting micro and nano patterns on electrospun fiber meshes at physiological temperatures, Small 9(20) (2013) 3405-3409.

[14] J. Lee, S.Y. Lee, J. Jang, Y.H. Jeong, D.-W. Cho, Fabrication of patterned nanofibrous mats using direct-write electrospinning, Langmuir 28(18) (2012) 7267-7275.

[15] C.H. Kim, Y.H. Jung, H.Y. Kim, D.R. Lee, N. Dharmaraj, K.E. Choi, Effect of collector temperature on the porous structure of electrospun fibers, Macromolecular Research 14(1) (2006) 59-65.

[16] P. Lu, Y. Xia, Maneuvering the internal porosity and surface morphology of electrospun polystyrene yarns by controlling the solvent and relative humidity, Langmuir 29(23) (2013) 7070-7078. 
[17] N. Sachot, O. Castaño, M.A. Mateos-Timoneda, E. Engel, J.A. Planell, Hierarchically engineered fibrous scaffolds for bone regeneration, Journal of The Royal Society Interface 10(88) (2013) 20130684.

[18] N.D. Luong, I.-S. Moon, D.S. Lee, Y.-K. Lee, J.-D. Nam, Surface modification of poly (I-lactide) electrospun fibers with nanocrystal hydroxyapatite for engineered scaffold applications, Materials Science and Engineering: C 28(8) (2008) 1242-1249.

[19] J. Chen, B. Chu, B.S. Hsiao, Mineralization of hydroxyapatite in electrospun nanofibrous poly (L-lactic acid) scaffolds, Journal of Biomedical Materials Research Part A 79(2) (2006) 307-317.

[20] Y. Zhang, J. Li, G. An, X. He, Highly porous SnO 2 fibers by electrospinning and oxygen plasma etching and its ethanol-sensing properties, Sensors and Actuators B: Chemical 144(1) (2010) 43-48.

[21] A. Nandakumar, Z.T. Birgani, D. Santos, A. Mentink, N. Auffermann, K. van der Werf, M. Bennink, L. Moroni, C. van Blitterswijk, P. Habibovic, Surface modification of electrospun fibre meshes by oxygen plasma for bone regeneration, Biofabrication 5(1) (2013) 015006.

[22] M.M. Demir, M.A. Gulgun, Y.Z. Menceloglu, B. Erman, S.S. Abramchuk, E.E. Makhaeva, A.R. Khokhlov, V.G. Matveeva, M.G. Sulman, Palladium nanoparticles by electrospinning from poly (acrylonitrileco-acrylic acid)-PdCl2 solutions. Relations between preparation conditions, particle size, and catalytic activity, Macromolecules 37(5) (2004) 1787-1792.

[23] A. Martins, E.D. Pinho, S. Faria, I. Pashkuleva, A.P. Marques, R.L. Reis, N.M. Neves, Surface modification of electrospun polycaprolactone nanofiber meshes by plasma treatment to enhance biological performance, Small 5(10) (2009) 1195-1206.

[24] L. Moroni, R. Licht, J. de Boer, J.R. de Wijn, C.A. van Blitterswijk, Fiber diameter and texture of electrospun PEOT/PBT scaffolds influence human mesenchymal stem cell proliferation and morphology, and the release of incorporated compounds, Biomaterials 27(28) (2006) 4911-4922.

[25] F. Zamani, M. Amani-Tehran, M. Latifi, M.A. Shokrgozar, The influence of surface nanoroughness of electrospun PLGA nanofibrous scaffold on nerve cell adhesion and proliferation, Journal of Materials Science: Materials in Medicine 24(6) (2013) 1551-1560.

[26] Q. Zhou, J. Xie, M. Bao, H. Yuan, Z. Ye, X. Lou, Y. Zhang, Engineering aligned electrospun PLLA microfibers with nano-porous surface nanotopography for modulating the responses of vascular smooth muscle cells, Journal of Materials Chemistry B 3(21) (2015) 4439-4450.

[27] M.F. Leong, K.S. Chian, P.S. Mhaisalkar, W.F. Ong, B.D. Ratner, Effect of electrospun poly (D, Llactide) fibrous scaffold with nanoporous surface on attachment of porcine esophageal epithelial cells and protein adsorption, Journal of Biomedical Materials Research Part A 89(4) (2009) 1040-1048.

[28] S. Liao, L.T. Nguyen, M. Ngiam, C. Wang, Z. Cheng, C.K. Chan, S. Ramakrishna, Biomimetic nanocomposites to control osteogenic differentiation of human mesenchymal stem cells, Advanced healthcare materials 3(5) (2014) 737-751.

[29] J. Rnjak-Kovacina, S.G. Wise, Z. Li, P.K. Maitz, C.J. Young, Y. Wang, A.S. Weiss, Tailoring the porosity and pore size of electrospun synthetic human elastin scaffolds for dermal tissue engineering, Biomaterials 32(28) (2011) 6729-6736.

[30] C.M. DiGirolamo, D. Stokes, D. Colter, D.G. Phinney, R. Class, D.J. Prockop, Propagation and senescence of human marrow stromal cells in culture: a simple colony-forming assay identifies samples with the greatest potential to propagate and differentiate, British Journal of Haematology 107(2) (1999) 275281. 
[31] A. Leferink, W. Hendrikson, J. Rouwkema, M. Karperien, v.C. Blitterswijk, L. Moroni, Increased cell seeding efficiency in bioplotted three-dimensional PEOT/PBT scaffolds, Journal of tissue engineering and regenerative medicine (2013).

[32] A. Nandakumar, H. Fernandes, J. de Boer, L. Moroni, P. Habibovic, C.A. van Blitterswijk, Fabrication of bioactive composite scaffolds by electrospinning for bone regeneration, Macromolecular bioscience 10(11) (2010) 1365-1373.

[33] S. Megelski, J.S. Stephens, D.B. Chase, J.F. Rabolt, Micro-and nanostructured surface morphology on electrospun polymer fibers, Macromolecules 35(22) (2002) 8456-8466.

[34] G.-T. Kim, J.-S. Lee, J.-H. Shin, Y.-C. Ahn, Y.-J. Hwang, H.-S. Shin, J.-K. Lee, C.-M. Sung, Investigation of pore formation for polystyrene electrospun fiber: effect of relative humidity, Korean Journal of Chemical Engineering 22(5) (2005) 783-788.

[35] J.L. Lowery, N. Datta, G.C. Rutledge, Effect of fiber diameter, pore size and seeding method on growth of human dermal fibroblasts in electrospun poly ( $\varepsilon$-caprolactone) fibrous mats, Biomaterials $31(3)$ (2010) 491-504.

[36] P. Kasten, I. Beyen, P. Niemeyer, R. Luginbühl, M. Bohner, W. Richter, Porosity and pore size of $\beta$ tricalcium phosphate scaffold can influence protein production and osteogenic differentiation of human mesenchymal stem cells: an in vitro and in vivo study, Acta Biomaterialia 4(6) (2008) 1904-1915.

[37] A.S. Badami, M.R. Kreke, M.S. Thompson, J.S. Riffle, A.S. Goldstein, Effect of fiber diameter on spreading, proliferation, and differentiation of osteoblastic cells on electrospun poly (lactic acid) substrates, Biomaterials 27(4) (2006) 596-606.

[38] U. Stachewicz, C.A. Stone, C.R. Willis, A.H. Barber, Charge assisted tailoring of chemical functionality at electrospun nanofiber surfaces, Journal of Materials Chemistry 22(43) (2012) 22935-22941.

[39] A.K. Dutta, K. Kamada, K. Ohta, Spectroscopic studies of nile red in organic solvents and polymers, J. Photochem. Photobiol. A: Chem. 93(1) (1996) 57-64.

[40] A.J. Magenau, J.A. Richards, M.A. Pasquinelli, D.A. Savin, R.T. Mathers, Systematic Insights from Medicinal Chemistry To Discern the Nature of Polymer Hydrophobicity, Macromolecules 48(19) (2015) 7230-7236.

[41] Y. Hou, A.M. Bardo, C. Martinez, D.A. Higgins, Characterization of molecular scale environments in polymer films by single molecule spectroscopy, The Journal of Physical Chemistry B 104(2) (2000) 212-219. [42] L. Ghasemi-Mobarakeh, M.P. Prabhakaran, M. Morshed, M.-H. Nasr-Esfahani, S. Ramakrishna, Electrospun poly ( $\varepsilon$-caprolactone)/gelatin nanofibrous scaffolds for nerve tissue engineering, Biomaterials 29(34) (2008) 4532-4539.

[43] A.A. Deschamps, M.B. Claase, W.J. Sleijster, J.D. de Bruijn, D.W. Grijpma, J. Feijen, Design of segmented poly (ether ester) materials and structures for the tissue engineering of bone, Journal of controlled release 78(1) (2002) 175-186.

[44] I. Wimpenny, N. Ashammakhi, Y. Yang, Chondrogenic potential of electrospun nanofibres for cartilage tissue engineering, Journal of tissue engineering and regenerative medicine 6(7) (2012) 536-549.

[45] Y.J. Chuah, Y. Zhang, Y. Wu, N.V. Menon, G.H. Goh, A.C. Lee, V. Chan, Y. Zhang, Y. Kang, Combinatorial effect of substratum properties on mesenchymal stem cell sheet engineering and subsequent multi-lineage differentiation, Acta biomaterialia 23 (2015) 52-62. 
[46] G. Wei, P.X. Ma, Partially nanofibrous architecture of 3D tissue engineering scaffolds, Biomaterials 30(32) (2009) 6426-6434.

[47] M.S. Lord, M. Foss, F. Besenbacher, Influence of nanoscale surface topography on protein adsorption and cellular response, Nano Today 5(1) (2010) 66-78.

[48] A.K. Gaharwar, S.M. Mihaila, A. Swami, A. Patel, S. Sant, R.L. Reis, A.P. Marques, M.E. Gomes, A. Khademhosseini, Bioactive silicate nanoplatelets for osteogenic differentiation of human mesenchymal stem cells, Advanced Materials 25(24) (2013) 3329-3336.

[49] Y. Wu, J.P. Zitelli, K.S. TenHuisen, X. Yu, M.R. Libera, Differential response of Staphylococci and osteoblasts to varying titanium surface roughness, Biomaterials 32(4) (2011) 951-960.

[50] M.-J. Kim, M.-U. Choi, C.-W. Kim, Activation of phospholipase D1 by surface roughness of titanium in MG63 osteoblast-like cell, Biomaterials 27(32) (2006) 5502-5511.

[51] G. Cadafalch Gazquez, H. Chen, S.A. Veldhuis, A. Solmaz, C. Mota, B.A. Boukamp, C.A. van Blitterswijk, J.E. ten Elshof, L. Moroni, Flexible Yttrium-Stabilized Zirconia Nanofibers Offer Bioactive Cues for Osteogenic Differentiation of Human Mesenchymal Stromal Cells, ACS nano (2016).

[52] B.C. Heng, T. Cao, E.H. Lee, Directing stem cell differentiation into the chondrogenic lineage in vitro, Stem cells 22(7) (2004) 1152-1167.

[53] S.C. Neves, C. Mota, A. Longoni, C.C. Barrias, P.L. Granja, L. Moroni, Additive manufactured polymeric 3D scaffolds with tailored surface topography influence mesenchymal stromal cells activity, Biofabrication 8(2) (2016) 025012.

[54] P.T. de Oliveira, A. Nanci, Nanotexturing of titanium-based surfaces upregulates expression of bone sialoprotein and osteopontin by cultured osteogenic cells, Biomaterials 25(3) (2004) 403-413.

[55] C.M. Curtin, G.M. Cunniffe, F.G. Lyons, K. Bessho, G.R. Dickson, G.P. Duffy, F.J. O'Brien, Innovative Collagen Nano-Hydroxyapatite Scaffolds Offer a Highly Efficient Non-Viral Gene Delivery Platform for Stem Cell-Mediated Bone Formation, Advanced Materials 24(6) (2012) 749-754.

[56] R.M. Nohutcu, L.K. McCauley, A.J. Koh, M.J. Somerman, Expression of extracellular matrix proteins in human periodontal ligament cells during mineralization in vitro, J. Periodontol. 68(4) (1997) 320-327.

[57] D. Khang, J. Choi, Y.-M. Im, Y.-J. Kim, J.-H. Jang, S.S. Kang, T.-H. Nam, J. Song, J.-W. Park, Role of subnano-, nano-and submicron-surface features on osteoblast differentiation of bone marrow mesenchymal stem cells, Biomaterials 33(26) (2012) 5997-6007.

[58] R.A. Gittens, T. McLachlan, R. Olivares-Navarrete, Y. Cai, S. Berner, R. Tannenbaum, Z. Schwartz, K.H. Sandhage, B.D. Boyan, The effects of combined micron-/submicron-scale surface roughness and nanoscale features on cell proliferation and differentiation, Biomaterials 32(13) (2011) 3395-3403.

[59] G. Abagnale, M. Steger, V.H. Nguyen, N. Hersch, A. Sechi, S. Joussen, B. Denecke, R. Merkel, B. Hoffmann, A. Dreser, Surface topography enhances differentiation of mesenchymal stem cells towards osteogenic and adipogenic lineages, Biomaterials 61 (2015) 316-326.

[60] R. Takács, C. Matta, C. Somogyi, T. Juhász, R. Zákány, Comparative analysis of osteogenic/chondrogenic differentiation potential in primary limb bud-derived and C3H10T1/2 cell linebased mouse micromass cultures, International journal of molecular sciences 14(8) (2013) 16141-16167.

[61] J.C. Robins, N. Akeno, A. Mukherjee, R.R. Dalal, B.J. Aronow, P. Koopman, T.L. Clemens, Hypoxia induces chondrocyte-specific gene expression in mesenchymal cells in association with transcriptional activation of Sox9, Bone 37(3) (2005) 313-322. 
[62] Q. Zhao, H. Eberspaecher, V. Lefebvre, B. De Crombrugghe, Parallel expression of Sox9 and Col2a1 in cells undergoing chondrogenesis, Developmental Dynamics 209(4) (1997) 377-386. 


\section{Surpporting Information}

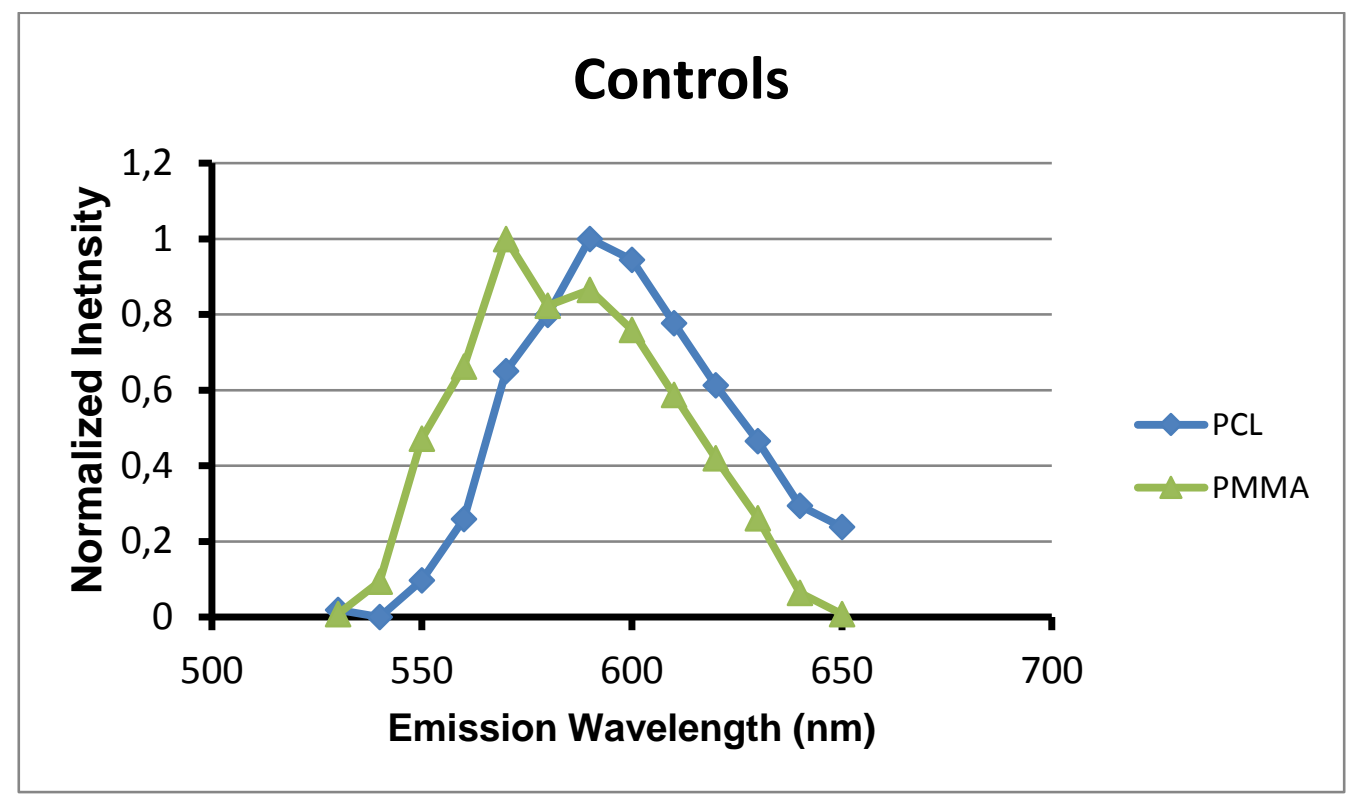

Figure S1. Nile Red emission profile of PCL and PMMA casting film.

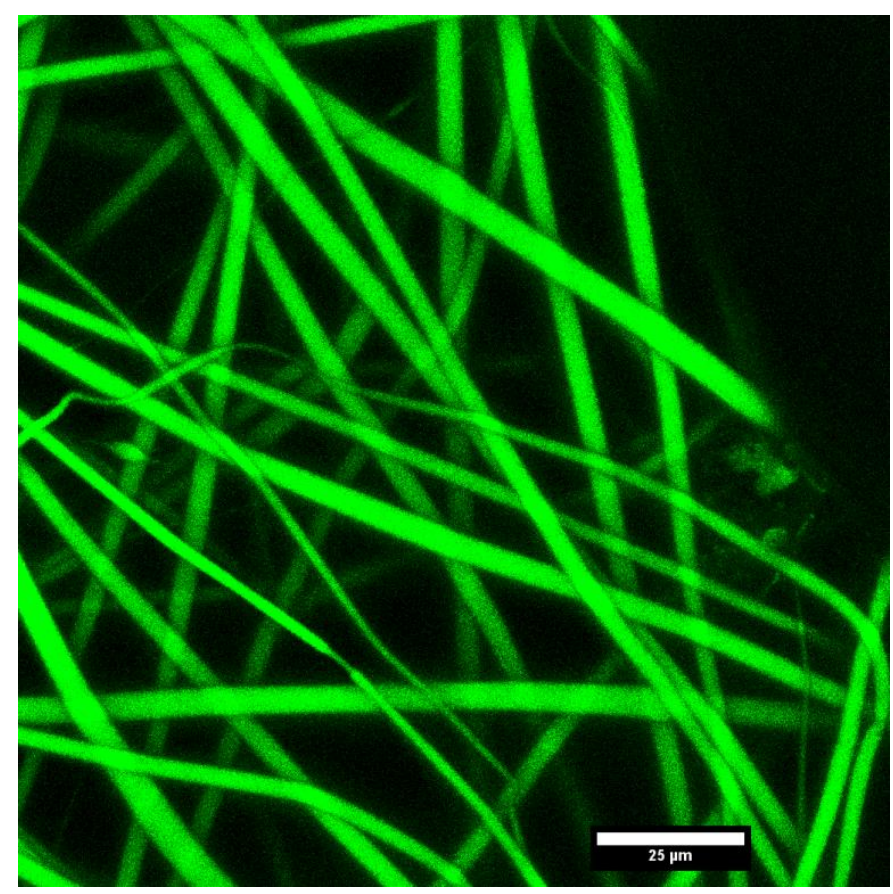

Figure S2. Representative of image of $20 \%$ humidity electrospun scaffold stained with Nile Red. $\lambda_{\mathrm{ex}}=514$. 
A

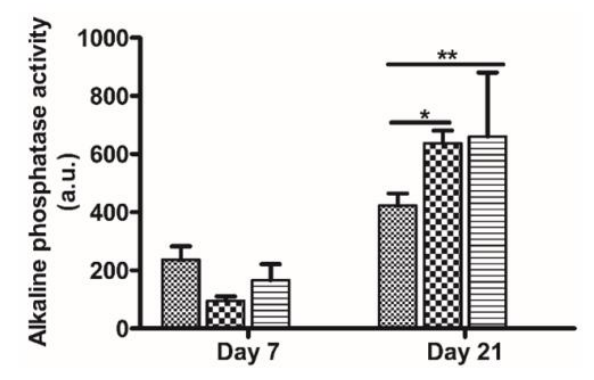

C

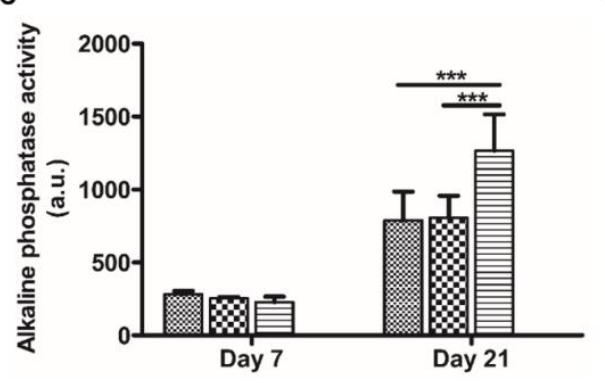

æ20\%
B

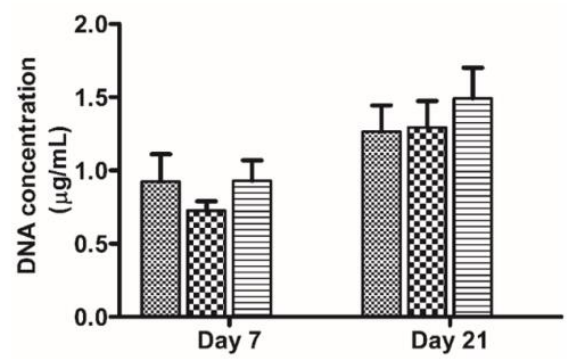

D

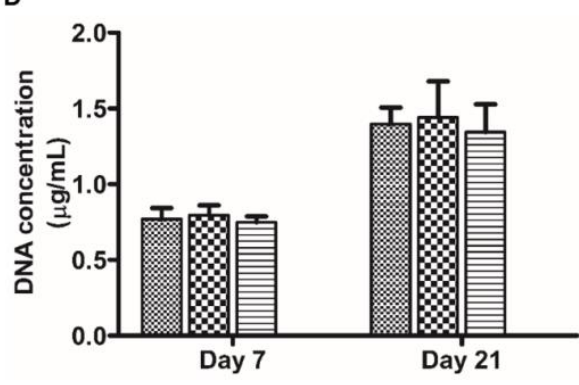

\$ $50 \%$ 曰 $70 \%$

Figure S3. ALP activity (A and C) and DNA concentration (B and D) of MSCs cultured in scaffolds in basic medium (A-B) and osteogenic medium(C-D).

A

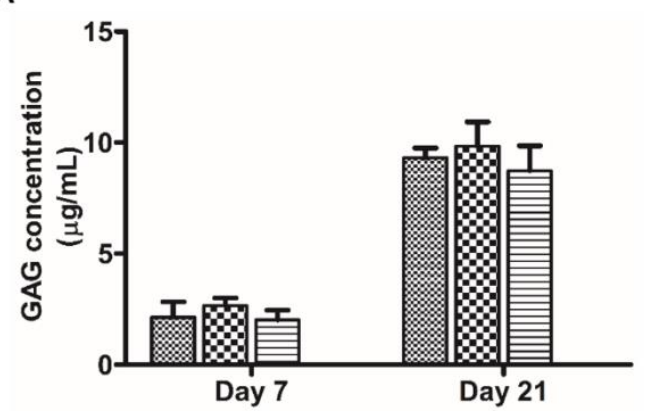

C

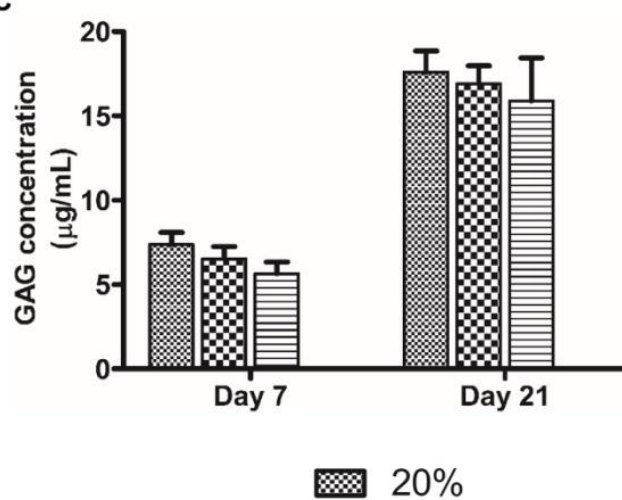

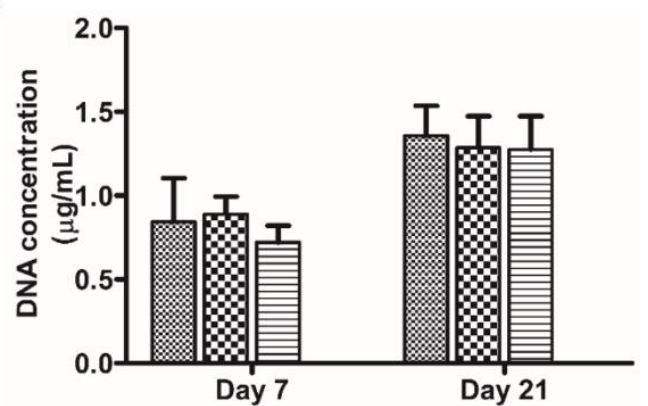

D

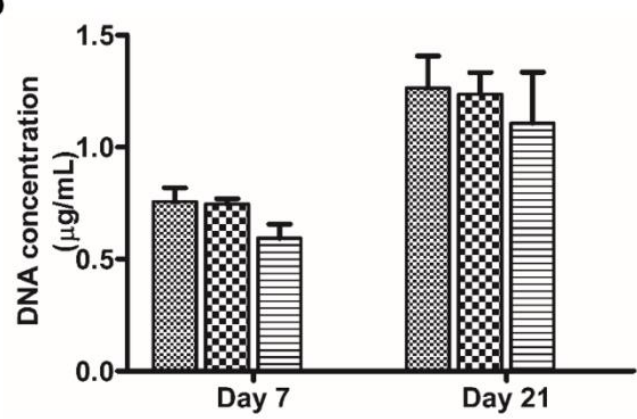

ए $50 \%$ 尼 $70 \%$

Figure S4. GAG activity ( $A$ and $C$ ) and DNA concentration ( $B$ and $D)$ of MSCs cultured in scaffolds in basic medium (A-B) and chondrogenic medium (C-D). 


\section{Chapter 5}

From Fiber Curls to Mesh Waves: a Platform for the Fabrication of Hierarchically Structured Electrospun Fibers Mimicking Natural Tissue Formation

Honglin Chen ${ }^{1,2}$, Danielle Ferreira Baptista ${ }^{1}$, Giuseppe Criscenti ${ }^{2}$, João Crispim², Hugo Fernandes ${ }^{2}$, Clemens van Blitterswijk ${ }^{1}$, Roman Truckenmüller ${ }^{1}$, Lorenzo Moroni ${ }^{1}$

${ }^{1}$ MERLN Institute for Technology-Inspired Regenerative Medicine, 6200 MD Maastricht, The Netherlands

${ }^{2}$ MIRA Institute for Biomedical Technology and Technical Medicine, University of Twente, 7500AE

Enschede, The Netherlands 


\begin{abstract}
Despite the tremendous progress in electrospinning in the past two decades, little progress has been made toward fabricating fibers having features mimicking the structure and architectural organization of extracellular matrix (ECM) fibrils and/or fibers in native tissue. Here, we present a simple, efficient, and versatile technique to produce curly or wavy patterns in electrospun fibers by using thermally shrinking materials. The pattern features could be easily manipulated by controlling various processing variables including the orientation of deposited fibers, the temporary content of sacrificial fibers, and the shape, geometry, chemistry and shrinking percentage of the thermally shrinking materials. In addition, our technique allows the retention of the imparted buckled pattern without the use of additional fixing agents or methods. Additionally, the technique allows fabrication of scaffolds with gradient and multiscale wavy patterns, thus mimicking the organization of multiple native tissues. Mechanical properties of the fabricated curly fibers could be varied through the shrinkage ratio of thermally shrinking materials, which enables the possibility to obtain a structure that could not only mimic the topographical patterns of connective tissues, but also mechanically resembles the properties of the same. We further investigated if the wavy patterns could regulate transforming growth factor-beta (TGF- $\beta$ ) production, a key signaling pathway involved in connective tissue development. Our results demonstrated that a TGF- $\beta$ reporter cell line cultured on wavy scaffolds had higher TGF- $\beta$ expression compared to non-wavy scaffolds. This platform may open up new opportunities in fabricating smart fibers for regenerative medicine.
\end{abstract}

\title{
Key words:
}

Electrospinning, shrink films, buckling, gradients, tissue regeneration 


\subsection{Introduction}

Extracellular matrix (ECM) fibrils or fibers with a curly or wavy microstructure are observed in several human tissues, such as ligaments, tendons, heart muscle, valve leaflets, cornea, iris, and intervertebral disk [1-4]. Typically, the crimped or wavy structure of native fibrous collagen has a periodicity in the range of 10 to $200 \mu \mathrm{m}$ [1]. Fibers with a crimped structure are capable of absorbing more deformation compared to their straight counterparts, and therefore show enhanced compliance at low strains while holding greater strength as the load increases[1,5]. Thus, collagen fibers containing a crimped morphology serve to buffer mechanical loads and enable the tissue to show nonlinear stiffness under increasing tensile strains[5].

Scaffold-based regenerative medicine has emerged as a promising strategy to repair damaged or diseased tissues. Currently, many techniques for generating scaffolds have been exploited such as phase separation, molecular self-assembly, electrospinning and additive manufacturing. Among these techniques, electrospinning has been widely explored due to its ability to produce fibrous meshes comparable to native extracellular matrix (ECM) in a simple and versatile manner [6, 7]. In addition, a desired pattern and structure of deposited electrospun fibers can be readily achieved by varying the collection methods, for example, randomly oriented fiber collected by a stationary plate, aligned fibers fabricated by a rotating mandrel or electrodes and tubular constructs produced using a rotating mandrel[6, 8-10]. Recently, electrospun scaffolds have been fabricated to mimic the native ECM environment in various degrees, particularly resembling the curly features. Younan and colleagues demonstrated the use of a plasticizer-based treatment, especially using ethanol, to generate a crimp structure along each electrospun fiber[5]. The morphology of crimp pattern obtained from their work is similar to other previous studies. Amsden et al. found that aligned electrospun fibers would self-crimp after removal from the mandrel at a temperature higher than the glass transition temperature of the spun polymer $[11,12]$. They also demonstrated that the degree of crimping could be varied by increasing the operating temperature or applying a plasticizer treatment on the polymer fibers. Using the same fabrication method, Brian and colleagues assessed the response of fibroblasts seeded on crimped-like structured scaffolds under dynamic mechanical loading[12]. Fibroblasts showed enhanced ECM synthesis and collagen accumulation in crimped structured scaffolds compared to uncrimped fibers [12]. Tonin and colleagues produced crimped polymer fibers utilizing a weak tangential air-flow fed 
from the top of a cylinder placed between the spinneret and the target collector[13]. Although the above mentioned methods have shown to mimic the wavy patterns observed in native ECM in different degree, the major drawbacks of these methods include limited control of the crimp pattern, relative complicated processing steps, the use of plasticizers potentially being toxic for the cells, and difficulties in generating multiscale and gradient wavy features, which are common features in native tissues.

Here, we propose a simple, versatile and efficient method that uses electrospinning combined with thermally shrinking materials, which tend to return to their original unstretched shape when heated above their softening point $\left(T_{s}\right)$. The thermally shrinking materials, such as corresponding films or tube-like semi-finished products, are typically either mono- or biaxially oriented according to their manufacturing protocol[14]. When the extending force is applied in one direction, monoaxial orientation occurs whereas when the stretching force is simultaneously applied in two directions, (equi)biaxial orientation is obtained. This causes it to shrink back toward its initial dimensions. By applying proper cooling, the molecules will be frozen in this state until sufficient heat energy is applied to allow the chains to shrink back. Using this new method, we are capable of fabricating architectures resembling the ones of collagen fibrils in various types of connective tissues including tendons and ligaments, muscles, skin and connective tissues of the eye.

\subsection{Materials and Methods}

\subsubsection{Depositing fibers on monoaxially oriented PLA shrink film}

Block copolymers PEOT/PBT (weight ratio of PEOT/PBT is 55/45; starting poly(ethylene glycol) (PEG) segments have a molecular weight of $300 \mathrm{Da}$ ) were purchased from PolyVation ${ }^{\circledR}$ (Groningen, The Netherlands). The polymer solution for electrospinning was prepared by dissolving PEOT/PBT in chloroform/1,1,1,3,3,3-hexafluoro-2-propanol (HFIP) $(\mathrm{v} / \mathrm{v}=4: 1)$ with a final concentration of $20 \%(\mathrm{w} / \mathrm{v})$, and stirred overnight at room temperature until complete dissolution. The solution was then loaded into a $5 \mathrm{~mL}$ syringe attached to a needle with a diameter of $0.8 \mathrm{~mm}$ and controlled by a syringe pump at a flow rate of $0.5 \mathrm{~mL} / \mathrm{h}$. A high voltage of $16 \mathrm{kV}$ was applied and the distance between the tip of the needle and the collector was $15 \mathrm{~cm}$. The temperature and relative humidity of the electrospinning chamber, which were kept the same for all electrospinning experiments, were controlled at $25^{\circ} \mathrm{C}$ and $30 \%$, respectively. Fibers were deposited on a oriented PLA film $(4 \mathrm{~cm} \times 4 \mathrm{~cm}$ ) which was placed on a conducting collector plate (for a random deposition) or two electrodes (for an aligned deposition). For collecting aligned 
fibers, changing the position of the PLA film during the electrospinning allows the deposition of fibers according to specific orientations: parallel, diagonal and perpendicular orientations relative to the direction of stretching and shrinkage were used in this experiment. The fiber density was controlled by controlling the deposition time. To know the influence of deposition orientation on curled patterns of fibers deposited in a low density, fibers were collected for $5 \mathrm{~s}$ on each PLA film. To investigate the effect of fiber density on curled patterns, fibers were collected up to $45 \mathrm{~min}$. Fiber-film constructs were then kept in an oven pre-heated to $75 \pm 1^{\circ} \mathrm{C}$ for 1 min to allow shrinkage of the PLA film or film frame, and formation of a buckled fiber mesh.

\subsubsection{Tailoring the buckled pattern by selective sacrificial fibers}

PVA was dissolved in ethanol/water $(\mathrm{v} / \mathrm{v}=1: 4)$ at a final concentration of $8 \%(\mathrm{w} / \mathrm{v})$ while the recipe for PEOT/PBT solution was the same as described above. To investigate low fiber densities, PEOT/PBT and PVA aligned fibers were deposited on a PLA film sequentially in a layer-by-layer way. The tip-to-collector distance and voltage applied, which were kept the same for the PEOT/PBT and the PVA solutions, were set to $15 \mathrm{~cm}$ and $16 \mathrm{kV}$, respectively. The feeding rates of the PEOT/PBT and the PVA solutions were $2 \mathrm{~mL}$ and $1 \mathrm{~mL} / \mathrm{h}$, respectively. To investigate the effect of sacrificial fibers on fiber meshes, PEOT/PBT and PVA fibers were electrospun simultaneously from two corresponding syringes and collected by a mandrel with a rotating speed of $300 \mathrm{rpm}$ to which a PLA film was attached. The two syringes were set up at either side of the mandrel with a tip-to-collector distance of $13 \mathrm{~cm}$. The feeding rates for PEOT/PBT and PVA were $5 \mathrm{~mL} / \mathrm{h}$ and $1 \mathrm{~mL} / \mathrm{h}$, respectively. The same voltage of $16 \mathrm{kV}$ was applied for both solutions.

Fiber-film constructs were then transferred to an oven pre-heated to $75^{\circ} \mathrm{C} \pm 1{ }^{\circ} \mathrm{C}$ for 1 min to allow shrinking of the PLA film and formation of a buckled fiber or fiber mesh. Finally, the fiber-film constructs were immersed in water for $30 \mathrm{~min}$ to remove PVA fibers.

\subsubsection{Influence of shrinking percentage of thermo-shrinkable material on curled features}

PEOT/PBT fibers were deposited on oriented PLA films which were placed on the top of a static collector. The fibers were collected for $30 \mathrm{~min}$. To investigate the influence of shrinking ratios of the films on the curly patterns, the fiber-film constructs were shrunk at $65^{\circ} \mathrm{C}$ for different time periods. 
5.2.4. Influence of shape and geometry of thermo-shrinkable materials on curled features

To fabricate a fiber mesh with a multiscale crimped pattern, a frame punched out from a PLA film was put on the top of a pair of electrodes to collect the fibers. The spinning parameters for PEOT/PBT were the same as described above. The fibers were collected for $10 \mathrm{~min}$.

To obtain a tubular curled scaffold, PEOT/PBT fibers were collected for 30 min using a mandrel wrapped with an oriented PLA film stripe and rotating with a speed of $150 \mathrm{rpm}$. The feeding rate of the polymer solution was $5 \mathrm{~mL} / \mathrm{h}$. The distance between the needle tip and the collector was set to $12 \mathrm{~cm}$ and the applied voltage was $16 \mathrm{kV}$. Fiber-film constructs were then transferred to an oven pre-heated to $75^{\circ} \mathrm{C} \pm 1{ }^{\circ} \mathrm{C}$ for 1 min to allow shrinking of the PLA film and formation of a buckled fiber or fiber mesh.

\subsubsection{Buckled PVA, PAN and PS fibers using PLA film}

PVA was dissolved in ethanol/water (1:4) at a concentration of $8 \%(\mathrm{w} / \mathrm{v})$ and stirred overnight before electrospinning. The applied voltage was $16 \mathrm{kV}$, the distance between needle tip and collector was $15 \mathrm{~cm}$ and the feeding rate of the polymer solution was 1 $\mathrm{mL} / \mathrm{h}$. Aligned fibers were collected using a pair of electrodes over which a PLA film was placed.

For electrospinning of PAN fibers, $10 \%$ polymer solution was prepared by dissolving PAN (MW=150,000, sigma) in dimethylformamide (DMF) and was stirred overnight at $60{ }^{\circ} \mathrm{C}$ to dissolve the polymer completely. The feeding rate of the polymer, the applied voltage, and the tip-to-collector distance was optimized at $1 \mathrm{~mL} / \mathrm{h}, 16 \mathrm{kV}$ and $15 \mathrm{~cm}$, respectively. Aligned fibers were collected using a pair of electrodes with PLA film on top of it. The fibers were collected for between $10 \mathrm{~s}$ and $5 \mathrm{~min}$.

PS (MW $=350,000$, from Sigma) was dissolved in DMF at room temperature under stirring overnight to obtain a $20 \mathrm{wt} \%$ homogeneous solution. The polymer solution was delivered at a rate of $0.5 \mathrm{~mL} / \mathrm{h}$. Electrospinning was carried out at $18 \mathrm{kV}$ and $15 \mathrm{~cm}$ distance from tip to collector. Fibers were deposited on the PLA film which was placed on top of a pair of electrodes. Fiber-film constructs were then transferred to an oven preheated to $75 \pm 1{ }^{\circ} \mathrm{C}$ for 1 min to allow shrinkage of the PLA film and formation of a buckled fiber mesh. 


\subsubsection{Buckled PEOT/PBT and PAN fibers using shrinkable PS material}

The electrospinning process for PEOT/PBT fibers was the same as described above except for the collection methods. For collecting random fibers, a biaxially oriented thermo-shrinkable PS film with a shrinking ratio of around $50 \%$ was put on top of a static plate collector. For collecting aligned fibers, the PS film was wrapped around a mandrel with a high speed of 5000 rpm. The thickness of fiber meshes was controlled by the deposition time.

The fabrication of PAN fibers was also the same as described above. PAN fibers were deposited on biaxially oriented thermo-shrinkable PS films placed on top of a pair of electrodes. The fibers were collected for $10 \mathrm{~s}$. Fiber-film constructs were then transferred to an oven pre-heated to $120{ }^{\circ} \mathrm{C}$ for $15 \mathrm{~min}$ to allow shrinkage of the PS film and formation of buckled fiber materials.

\subsubsection{Characterization of buckled fibers}

To check the morphology of the scaffolds, the samples were sputter-coated with gold and then observed under a scanning electron microscopy (SEM) (Philips XL-30) at an accelerating voltage of $10 \mathrm{kV}$. For the low density mats, the wavelength and amplitude were measured using Image J; 40 fibers were measured for each condition (5 images with 8 fibers each). The wavelength of the curly features was considered as the distance between two consecutive peaks; the amplitude was measured as the distance between the maximum and minimum of the wave divided by two.

Uniaxial tensile tests were performed to evaluate the mechanical properties of the different curled structures. Six different percentages of shrinkage $(10,25,30,40$ and $50 \%$ ), obtained via varying the exposition time to a shrinkage temperature, were analysed while non-curly fibers were used as control. A total of 7 samples for each type were tested. The samples were given a rectangular shape using a surgical scalpel so that the length-to-width aspect ratio (4:1) provided uniform tensile stress in the region where the strain was measured. All the samples had a constant cross-sectional area (CSA) after shrinking, which was measured with a digital calliper (accuracy of $0.02 \mathrm{~mm}$ and resolution of $0.01 \mathrm{~mm}$ ) and we assumed it had a rectangular shape. The samples were fixed in a standard clamps and axially aligned to the $500 \mathrm{~N}$ load cell of a Zwick Z020 materialtesting machine. The scaffolds were preconditioned by a series of 10 cycles where they were exposed to $3 \%$ of strain at a strain rate of $0.1 \% / \mathrm{s}$, in order to reduce hysteresis. The samples were then pulled until failure at a strain rate of $0.3 \% / \mathrm{s}$. With CSA and strain 
measurements, a stress-strain curve representing the tensile mechanical properties of the different scaffolds was created. From the stress-strain curves, the following parameters were obtained: Young's Modulus $(\mathrm{Pa})$, defined as the slope of the linear region of the stress-strain curve, ultimate stress $(\mathrm{Pa})$, ultimate strain (\%) and strain energy density (Pa) at failure. The failure modes were also noted.

\subsection{8. hMSC culture and seeding}

Pre-selected hMSCs (donor No. 8001L) were obtained from the Institute of Regenerative Medicine at Texas A\&M University. Briefly, a bone marrow aspirate was drawn and mononuclear cells were separated using density centrifugation. The cells were plated to obtain adherent hMSCs, which were harvested when cells reached 60-80\% confluence. These were considered passage zero (P0) cells. These P0 cells were expanded, harvested and frozen at passage 1 (P1) for distribution. hMSCs were thawed and expanded in T-flasks till reaching $80 \%$ confluence in basic medium composed of Minimum Essential Medium (a-MEM, Gibco), 10\% FBS (Lonza), 2 mM L-glutamine (Gibco), $0.2 \mathrm{mM}$ ascorbic acid (Sigma), $100 \mathrm{U} / \mathrm{mL}$ penicillin and $100 \mathrm{mg} / \mathrm{mL}$ streptomycin (Gibco). Samples were punched to fit a 48 -well plate. After sterilization with $70 \%$ ethanol for 15 min for 2 times, the scaffolds were washed with phosphate-buffered saline (PBS) 3 times, afterwards incubated in basic medium for 3 hours at $37^{\circ} \mathrm{C}$ before cell seeding. Cells were seeded in scaffolds with a cell density of 5000 cells $/ \mathrm{cm}^{2}$. The cell culture was carried on for 5 days with medium replacement every 2 days.

\subsubsection{Cell penetration analysis}

The samples were fixed with formalin for 30 minutes and washed with PBS. To get crosssections, samples were cut in half and embedded with Cryomatrix ${ }^{\mathrm{TM}}$ mounting media on mounting blocks with the cross-section facing upwards and $8 \mu \mathrm{m}$ cross-sections were obtained using a cryotome (Shandon Cryotome, Thermo Scientific). To avoid polymer auto-fluorescence, the cross-sections were stained with $1 \%(\mathrm{w} / \mathrm{v})$ Sudan Black solution in $70 \%$ ethanol, incubated for 1 hour at room temperature, washed thoroughly with PBS for $5 \mathrm{~min}$ for 5 times. To visualize cell penetration into the scaffold, cross-sections were stained with DAPI. Briefly, samples were incubated with $1 \%$ Triton $\mathrm{x}-100$ for 10 min on ice, washed with PBS and blocked with bovine serum albumin $(10 \mathrm{mg} / \mathrm{mL})$ for 60 minutes to block unspecific binding. After removal of this solution, DAPI (1:100 PBS) was added for $15 \mathrm{~min}$ at room temperature avoiding light. Finally, all samples were washed with PBS 
and kept at $4{ }^{\circ} \mathrm{C}$ in the dark. Images were then taken using a fluorescent microscope (Nikon Eclipse E600).

To measure the penetration depth associated with each cell in each cross-section, the pictures were analyzed using Image $\mathrm{J}$. The approach taken was to define the borders of the section and then to create a grid for each image which divided the height of the crosssection in 5 bins (Figure S9), corresponding to regions from the top to the bottom of the scaffold. The first bin was considered the top of the scaffold while the last bin was identified as the bottom of the scaffold. To normalize the data, the number of cells in each bin was divided by the total amount of cells in that cross-section and the values presented in the $x$-axis were presented as the ratio of infiltration depth to the thickness of scaffold.

\subsubsection{MLEC culture and seeding}

MLEC, a kind gift from Dr. Daniel Rifkin from the Department of Cell Biology at the New York University School of Medicine, were stably transfected with an expression construct containing a truncated plasminogen activator inhibitor-1 (PAl-1) promoter fused to the firefly luciferase reporter gene. These cells are highly sensitive and specific for TGF- $\beta$ production based on its ability to induce PAI-1 expression. MLEC were thawed and expanded in T-flasks till reaching 80\% confluence in Dulbecco's Modified Eagle's Medium a (DMEM- $\alpha$ ) supplemented with 10\% FBS, $0.2 \mathrm{mM} \mathrm{L-glutamine,} \mathrm{and} 100$ units $/ \mathrm{mL}$ penicillin and $100 \mathrm{mg} / \mathrm{mL}$ streptomycin. The samples were punched to fit a 96 -well plate (Thermo Scientific). After sterilization with $70 \%$ ethanol for 15 min for 3 times, the scaffolds were incubated in the described medium (without TGF- $\beta 1$ ) overnight. The next day, cells were seeded with a cell density of $50,000 \mathrm{cells} / \mathrm{cm}^{2}$ on the scaffolds and on tissue culture plates used as a control. Cell culture was carried on for 5 days.

\subsubsection{Luciferase and DNA assay}

After removing the medium, samples were washed thoroughly with PBS, lysis buffer ( $5 x)$ was added and diluted (5:1) in Milli-Q water. Samples were stored at $-80^{\circ} \mathrm{C}$ for at least $1 \mathrm{~h}$, then defrosted on a plate shaker at room temperature. Each sample was divided, one half for the Luciferase and the other half for the DNA assay and transferred into 2 different, white and black 96-well plates, respectively. For the Luciferase assay, Luciferase Assay Substrate (Promega) was added to the samples and the light produced was read on a plate reader (Victor 3 TM, PerkinElmer ${ }^{\circledR}$ ) column by column. 
The DNA assay was performed using the CyQUANT® cell proliferation assay kit (Molecular Probes, Eugene, USA) according to its manufacture manual. Briefly, samples were supplemented with lysis buffer containing RNAse (Sigma) and incubated for $1 \mathrm{~h}$ on a plate shaker, meanwhile a standard curve was prepared using $\Lambda$ DNA standard (provided from the CyQUANT kit). After the incubation period, CyQUANT® GR dye in lysis buffer was added to the samples and the fluorescence was measured on a plate reader (Victor $3 \mathrm{TM}$, PerkinElmer ${ }^{\circledR}$ ) at an excitation wavelength of $480 \mathrm{~nm}$ and an emission wavelength of $520 \mathrm{~nm}$.

\subsubsection{Statistical Analysis}

Statistical differences were determined using GraphPad Prism 5.001 software (San Diego, USA). Statistical differences between groups were determined using analysis of variance (ANOVA) followed by a Tukey's post-hoc test unless otherwise mentioned. Statistical significance between the control group and the experimental groups are indicated by $\left(^{*}\right)$ which represents a $p$-value $<0.05$, by ${\left({ }^{* *}\right)}$ which represents a $p$-value $<$ 0.01 , and by $\left({ }^{* * *}\right)$ which represents a $p$-value $<0.001$. Values are presented as mean \pm standard deviation.

\subsection{Results and discussion}

5.3.1. Fabrication of curly fibers 


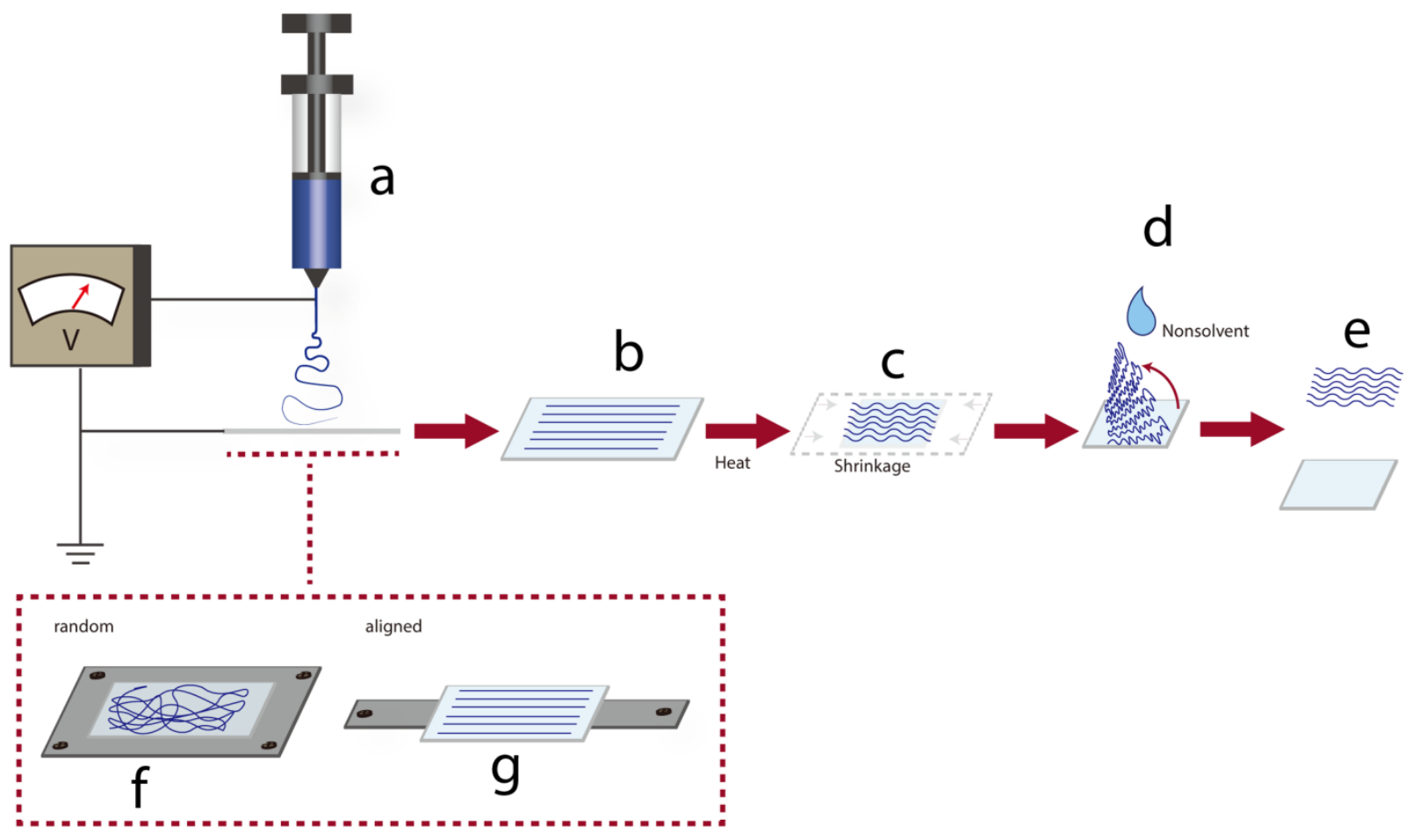

Figure 1. Schematic of a typical process for generating curled patterns in electrospun fibers using thermal shrinkage. (a) Depositing electrospun fibers over a thermo-shrinkable film. (b) Fiber-film construct before thermal shrinkage. (c) Wavy fibers form after thermal shrinkage. (d) and (e) Wavy fibers are detached from the thermally shrinkable film using a non-solvent. ( $f$ ) Random fibers are collected through using a static ground plate. (g) Aligned fibers are obtained using a pair of electrodes.

Figure 1 shows the schematic of producing curly fiber. In a typical process, polymer fibers are deposited onto thermo-shrinkable materials (e.g. a corresponding planar film) via electrospinning (Figure 1a and b). By heating the construct above $T_{s}$, the fibers buckle as a consequence of the substrates shrinking while the fibers adhering to them. Cooling down the construct at room temperature leads to the thermal fixation of the curled pattern generated in the electrospun fibers, and this is one of the main advantages of our technique compared to previous studies, where an extra fixation agent or method was always required[1,5]. In the present work, a block copolymer poly(ethylene oxide terephthalate)/poly(butylene terephthalate) (PEOT/PBT), which has been demonstrated as a biocompatible and -degradable polymer, was used as a material for electrospinning[15, 16]. In addition, a monoaxial poly(lactic acid) (PLA) film having a $T_{s}$ around $65 \stackrel{\circ}{\circ}$ was utilized as the main thermo-shrinkable material. To determine its shrinking properties, three different temperatures $\left(65,75\right.$ and $85{ }^{\circ} \mathrm{C}$ ) were tested during different periods of time (Figure S1). According to these results, standard values for the 
film shrinkage were set at $75^{\circ} \mathrm{C}$ for 1 minute which allowed $60 \%$ shrinkage of the PLA film and enough time to control the process manually.

Importantly, the applied $T_{s}$ of thermo-shrinkable materials should be below the melting temperature $\left(T_{m}\right)$ of the polymer used for fabricating the fibers. Figure $\mathrm{S} 2$ shows poly(caprolactone) (PCL) fibers which became partially melted after shrinking the PLA film onto which they have been deposited. No curls were formed because the $T_{m}$ of PCL (around $60^{\circ} \mathrm{C}$ ) is lower than the $T_{s}$ of the PLA film.

\subsubsection{From single fiber curls to multiscale mesh waves}

Random fibers were collected using a static ground plate, while aligned fibers were generated using a pair of parallel electrodes. Changing the angle of the PLA film during electrospinning allows deposition of fibers relative to specific film orientations (Figure. 2a). In case of a monoaxially oriented film, a perpendicular configuration corresponds to deposit fibers at a $90^{\circ}$ angle with respect to the shrinkage axis; a parallel configuration corresponds to deposit fibers along the shrinkage axis; a diagonal configuration corresponds to fiber deposition at a $45^{\circ}$ angle; a random configuration corresponds to randomly oriented fibers. As shown in Figure $2 \mathrm{~b}$, after shrinking the PLA film, curled features were generated in fibers of all deposition direction except for the perpendicular one. The perpendicular configuration only allowed fiber compactness, because the space between each fiber decreased as the film shrunk, bringing the fibers closer together. The wavelengths and amplitudes of curls for parallel configuration showed a significant difference compared to that of diagonal configurations (Figure 2c). However, it is difficult to calculate the wavelengths and amplitudes for the random configuration, since the fibers were randomly deposited and crossed each other, leading to heterogeneous curls in fiber. 


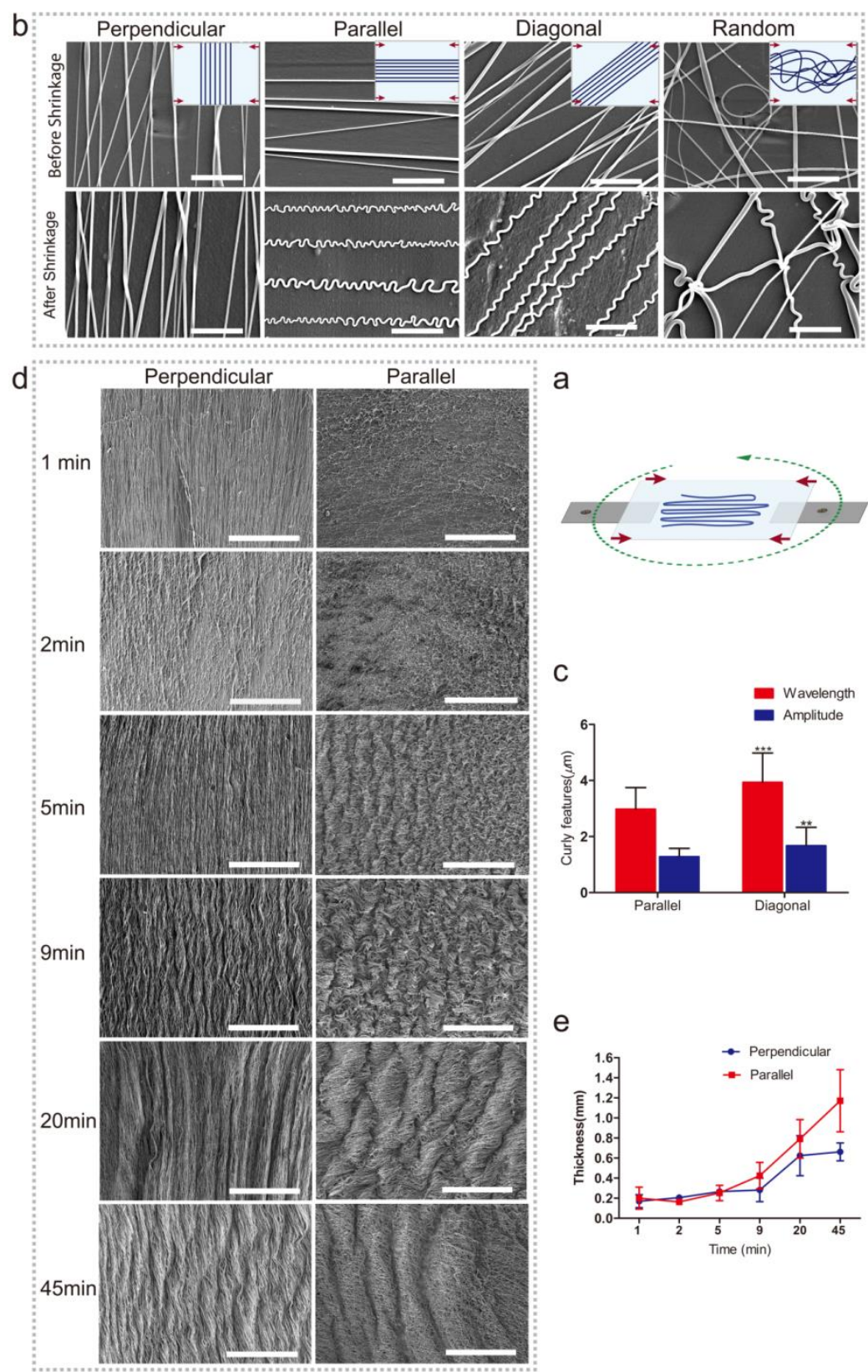

Figure 2. From single fiber curls to multiscale mesh waves. (a) A schematic illustration of varying fiber orientations by changing the position of the film over the electrodes. Red arrows indicate the shrinking direction of the oriented PLA film, while the green circular arrow means rotating the film to achieve different fiber orientations. (b) Comparison between fibers deposited in different directions relative to the direction of shrinkage before and after shrinkage. (c) Histogram of the wavelength and amplitude for parallel and diagonal configurations. (d) Topographical evolution as the time of deposition increased and with it the mesh thickness. Samples where fibers were deposited perpendicular to the direction of shrinkage showed waviness rather than only curls on individual fibers after 9 minutes of deposition, whereas the perpendicular samples show waviness after 5 minutes. (e) Depicting the thickness of samples with fibers deposited perpendicular or parallel to the shrinkage axis, after shrinking, according to time of deposition. Scale bars: $20 \mu \mathrm{m}$ (b) and $500 \mu \mathrm{m}$ (d). ${ }^{*} \mathrm{p}$-value $<0.1,{ }^{* *} \mathrm{p}$ - value $<0.01$ and ${ }^{* * *} \mathrm{p}$-value $<0.001$. 
With the same process, it is possible to transfer the effect of generating curls on single fibers to the effect of generating multiscale waves on fiber meshes. The transition between the two effects relied on the density of deposited fibers. The density of deposited fibers in turn depends on the time of spinning; longer times correspond to a thicker fiber deposition, leading to the formation of fiber meshes evolved from low density fibers. For the given fiber chemistry and dimension in the present study, the multiscale waves on perpendicular samples started at 9 min deposition time with an average thickness of $0.3 \pm$ $0.1 \mathrm{~mm}$, whilst for the parallel samples it occurred at $5 \mathrm{~min}$ with the same average thickness of $0.3 \pm 0.1 \mathrm{~mm}$ (Figure $2 \mathrm{~d}$ and e). Although previous methods have proved capable of generating curled patterns on single fibers, they have no way of controlling the transition between generating curls on single fiber and generating multiscale waves on fiber meshes. With the present technique, the fabricated scaffolds can assume different structural configurations, which are promising as they may mimic the hierarchical organization of native tissues from the nano- to the macro-scale. The presented platform works also for other polymer fibers. As shown in Figure S3 and Figure S4, curly fibers of polyvinyl alcohol (PVA), polystyrene (PS) and polyacrylonitrile (PAN) could be generated using the oriented PLA films. Besides oriented PLA, the present technique could also be translated to other thermo-shrinkable materials. Theoretically, every thermoplastic material can be made into a stretched, oriented film, the frozen stress of which can be unlocked resulting in shrinking when arriving at the stretching temperature again, for instance, oriented PS films. Figure S5 and Figure S6 show that curly features were successfully generated in PEOT/PBT and PAN fibers, respectively, using an equibiaxially shrinkable PS film.

\subsubsection{Maneuvering curled pattern using additional sacrificial electrospun fibers}

The curled pattern of fibers could also be tailored by using sacrificial fibers, such as those made from water soluble polymers like PVA. Figure 3a shows the schematic of fabricating a mixture of PVA and PEOT/PBT (PVA-PEOT/PBT) fibers, where PVA fibers were firstly deposited on the PLA film, followed by PEOT/PBT fibers. After removal of the sacrificial PVA fibers, PEOT/PBT fibers held a distinguishably different curled pattern having both periodical larger arches and small curls within the same fiber, which did not appear in the absence of the sacrificial polymer. However, the removal of the sacrificial polymer was distinguishable whether it was the first or second layer. As shown in Figure S7a-c, the final PEOT/PBT-PVA fibers in which the PVA was deposited as a second layer have a 
different curly morphology after removing PVA fibers. The comparison of curled features between final PVA-PEOT/PBT and PEOT/PBT-PVA fibers is summarized in Figure S7d. The wavelength and amplitude for the larger arches in PVA-PEOT/PBT fibers were $47.2 \pm$ 22.9 and $6.2 \pm 1.8 \mu \mathrm{m}$, respectively, which is similar to the values of crimped patterns in human ligament collagen (wavelength of $45-60 \mu \mathrm{m}$ and amplitude of 5-10 $\mu \mathrm{m}$ ) [11]. On the other hand, the corresponding values for smaller curls in PVA-PEOT/PBT fibers were $5.2 \pm 1.6$ and $1.9 \pm 0.5 \mu \mathrm{m}$, respectively. In the case of PEOT/PBT-PVA fibers, they had an average wavelength of $3.2 \pm 1.3 \mu \mathrm{m}$ and average amplitude of $1.4 \pm 0.5 \mu \mathrm{m}$, which is significantly different $(p<0.01)$ from the PVA-PEOT/PBT fibers. Previous methods used to curl electrospun fibers could produce similar patterns, to the ones achieved in the present work. [11, 17, 18]. For example, Tao Han et al. produced bucked electrospun fibers through manipulating the buckling instability of the electrospinning jet. The wavelength range corresponding to buckled pattern in their study was 1.2-30.6 $\mu \mathrm{m}$ [17], which range was comparable to the ones produced in the present work (from $3.2 \pm 1.3$ $\mu \mathrm{m}$ up to $47.2 \pm 22.9 \mu \mathrm{m}$ ). Based on a complex method of calcination and reduction of copper, Guoquing Chang et al. produced 3D helical ribbons with fibers presenting a wavelength of $3.2 \mu \mathrm{m}[18]$.

This strategy of using a sacrificial polymer could also be translated to fiber meshes for tuning their surface topography. It has been demonstrated that topographical cues are important to cellular behaviors, including cell morphology, proliferation, migration and differentiation [19, 20]. Figure 3b shows the comparison between meshes before and after immersion in water. A difference in surface topographical pattern between them was visible, inclusively a smaller fraction structure after immersion due to the removal of the PVA fibers. A similar phenomenon was also referred in previous work of Baker et al. where they combined electrospinning with sacrificial fibers to generate a scaffold having engineered delamination [21]. Taken together, through selective removal of fibers, it is possible to modulate the wavy or curled patterns on single fibers as well as fiber meshes. 

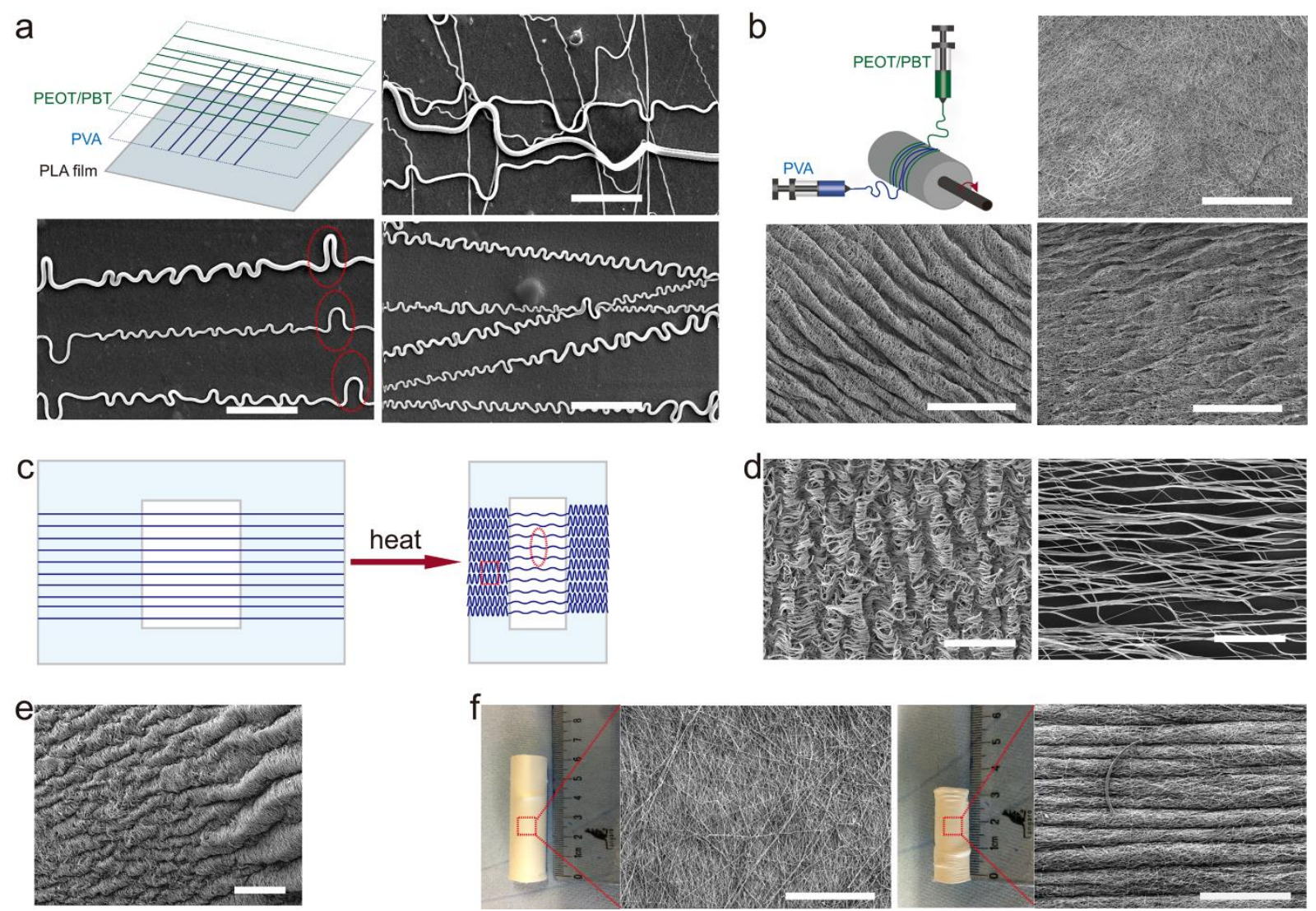

Figure 3.Tayloring curled features by using additional sacrificial electrospun fibers or tuning thermoshrinkable materials. (a) Tayloring curled patterns along fibers by sacrifying a second layer of fibers from poly(vinyl alchol) (PVA). Illustration of fiber layer deposition (top left); SEM image of curled PEOT/PBT-PVA fibers before (top right) and after (bottom right) sacrifying the PVA fibers; morphogy of curled PEOT/PBT fibers without using a sacrificial layer of PVA fibers (bottom left). (b) Changing wavy patterns of fiber mesh by using scarificial PVA fibers. Schematic figure of fabricating a PEOT/PBT-PVA blending fiber mesh (top left); PEOT/PBT-PVA fiber mesh before (top right) and after (bottom left) shrinking; morphology of the wavy PEOT/PBT-PVA fiber mesh after sacrificing the PVA fibers (bottom right). (c) Schematic of fabricating fibers having multiscale wavy patterns. (d) Morphology of curled pattern in a square outlined area (left) and in encircled outlined area (right) in c. (e) SEM image of electrospun fiber having a gradient waviness. (f) Fabricating a buckled fiber mesh mimicking the structure of the trachea by using PLA shrink tubes. Morphology of PEOT/PBT fibers deposited on PLA tubes before (left) and after (right) shrinking. Scale bar: $20 \mu \mathrm{m}(\mathrm{a}), 100 \mu \mathrm{m}(\mathrm{d})$, and $500 \mu \mathrm{m}$ (b, e and f).

\subsubsection{Influence of shrink properties and geometric shape of thermally shrinkable} materials on curled features

As demonstrated in Figure S1, the shrinkage ratio of thermo-shrinkable materials is time dependent. Based on this concept, the curled features could be regulated via manipulating shrinkage ratios (Figure S8). When the shrinkage ratio increased from 10\% to $40 \%$, the amount of "wrinkles" in scaffolds increased, whereas the average wavelength 
of the "wrinkles" decreased correspondingly. The structure and geometry of thermoshrinkable materials could be designed for specific requirement. Figure $3 \mathrm{c}$ shows an electrospun mesh having multiscale buckled patterns generated using a planar oriented PLA film with an open central area. When the fiber mesh shrunk, the fibers possessed a lower ratio of shrinkage on the area where there was no film underneath compared to the area where fibers adhered to the film. Thus, multiscale buckled patterns could be generated on the fiber mesh. After shrinking, the fiber mesh had a coiled pattern on the sides, in contrast to a smoother wavy pattern in its central zone (Figure 3d). Native tissue interfaces, such as the corresponding regions of tendons, ligaments and bone show gradients in structure, composition and mechanics [22, 23]. In the specific case of the interface between tendon or ligaments with bone, a gradient in mechanical properties serves to mitigate stress concentrations [24]. Interestingly, with one corner of the oriented PLA film clamped and thus hindering from shrinking, a gradient wavy pattern could be generated (Figure 3e). A scaffold having such gradient waviness could be used in the field of interface tissue regeneration. Figure $3 f$ shows electrospun fibers deposited on a tubular oriented PLA film. After shrinking, a corrugated hose-like construct mimicking the structure of a trachea segment of $104 \pm 22.5 \mu \mathrm{m}$ could be fabricated.

Overall, a desirable curly feature could be achieved via manipulating the shrink properties of thermally shrinking materials and their geometric shapes in particular. Currently, it is able to design the geometric shapes of thermally shrinking materials into a variety of stretch configurations, such as tubes and more complex geometries (e.g., through thermoforming of films or blow molding of tubes), than only mono- or (equi) biaxially oriented films.

\subsubsection{Mechanical properties}

The mechanical behavior of the 3D waves was evaluated performing uniaxial tensile tests. Scaffolds showed a limited hysteresis on the preconditioning phase. During the uniaxial tensile testing, stress-strain curves representing the mechanical properties of the structure were obtained. The curves show a stress-strain behavior characterized by a non-linear toe region between 0 and around $1 \%$ of strain, a linear region between around $1 \%$ and $12 \%$, and a final region where the curve reached the ultimate stress. The obtained results are listed in Table S1 and Figure 4a. A statistically significant decrease of the Young's modulus with the increase of the shrinking percentage was detected (Figure 4b). Similar trends were found for the ultimate stress, ultimate strain and strain 
energy density (Figure 4c-e). In particular, the $25 \%$ of shrinkage seemed to represent a threshold in the case of the ultimate stress and strain energy density.

In order to describe the mechanical behavior of the different curled networks, the experimental data obtained from the mechanical tensile tests were analyzed through a mathematical model. Hyperelastic models are commonly used to describe the behavior of skin, other soft-tissue and rubber-like materials because of their elastic behavior[25]. Here, the 3-parameter Mooney-Rivlin model was used to describe the material's stressstrain relationship as a function of the empirically determined constants $C_{01}, C_{10}$ and $C_{20}$. Considering a uniaxial tensile test, the model can be expressed as

$$
\sigma=2 C_{10}\left(\lambda-\frac{1}{\lambda^{2}}\right)+2 C_{01}\left(\lambda-\frac{1}{\lambda^{3}}\right)+4 C_{20}\left(\lambda-\frac{1}{\lambda^{2}}\right)\left(\lambda^{2}+\frac{2}{\lambda}-3\right)
$$

where $\sigma$ is the applied stress, $C_{10}, C_{01}$ and $C_{20}$ are material parameter constants, and $\lambda$ is the stretch ratio, defined as $\lambda=1+\varepsilon$. The estimation of the model parameters is provided in the Supplementary Table S2 and Figure S11 in the Supplementary Note.

The use of the Mooney-Rivlin model in case of a uniaxial tension condition allowed to fit the experimental data obtaining three different adimensional constants. The statistical differences among the different parameters demonstrated a different mechanical behavior between the parallel networks and the ones with different percentage of shrinkage. 

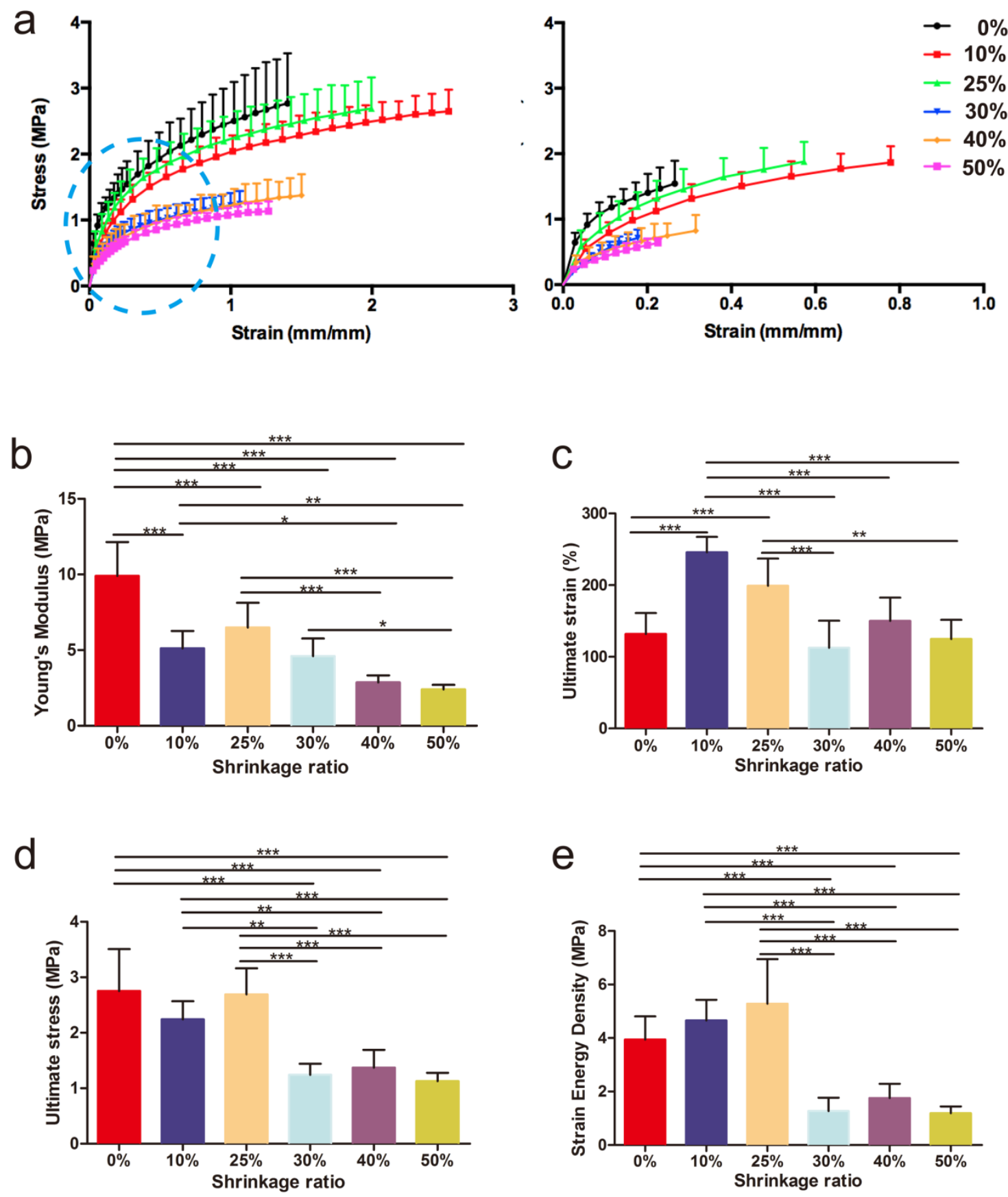

Figure 4. Mechanical properties of wave structures. (a) Figures of stress-strain behavior (left) and zoom in the encircled outlined area in left (right). (b) Young's modulus. (c) Ultimate stress. (d) Ultimate strain. (e) Strain energy density.

\subsubsection{Cell penetration}

Cell penetration and growth throughout the scaffold are vitally important for tissue engineering applications[26]. Although electrospinning scaffolds have drawn much interest in the tissue engineering field, one major limitation is that their fibers are typically densely packed, therefore hampering cellular infiltration into the scaffold [26, 27]. Thus, 
many approaches have been explored to overcome this problem, for instance sacrificial polymers electrospinning, salt leaching, and electrospinning in combination with electrospraying [26]. However, the current methods to improve cell infiltration reduce scaffold stability [21, 28]. Here, we hypothesized that wavy fibers with a larger void space into their porous structure will enhance cell penetration into electrospun scaffolds. Human mesenchymal stromal cells (hMSCs) were seeded the same density on flat and wavy scaffolds in basic medium. SEM images revealed that hMSCs on flat scaffolds are likely to disperse on its surface while a big portion of cells on wavy fibers tend to entangle within its fibers (Figure $5 \mathrm{a}$ and $\mathrm{b}$ ). To further investigate their infiltration and growth into flat and wavy scaffolds, nuclear staining was performed and cross-sections were analyzed. hMSCs on the flat scaffolds did not migrate further than superficial layers, whereas hMSCs on the wavy scaffolds migrated deeper and faster into the scaffold (Figure $5 b$ ). The distribution of cells in each depth section of flat and wavy scaffolds is summarized in Figure $5 \mathrm{c}$ and $\mathrm{d}$. For comparison, the depth presented in the $\mathrm{x}$-axis corresponds to an average ratio of infiltration depth to the thickness of scaffolds, since the wavy scaffolds had a higher thickness compared to the flat scaffolds, as a consequence of the buckling effect occurring during the underneath film shrinking (Figure S9b). By day 2, the largest amount of cells stayed at the top of the scaffolds and decreased in number towards the bottom of the scaffolds for both wavy and flat substrates. Despite presenting the same trend, flat scaffolds presented a higher concentration of cells in the upper region whereas wavy scaffolds showed a more homogeneous distribution. After 5 days of culture, more cells migrated into the deeper regions of both scaffolds. However, more cells were present in the deeper region of the wavy scaffolds compared to the flat ones. These results thus indicate that wavy scaffolds support a more homogenous distribution of cells throughout their thickness and a faster cell migration than flat scaffolds. 

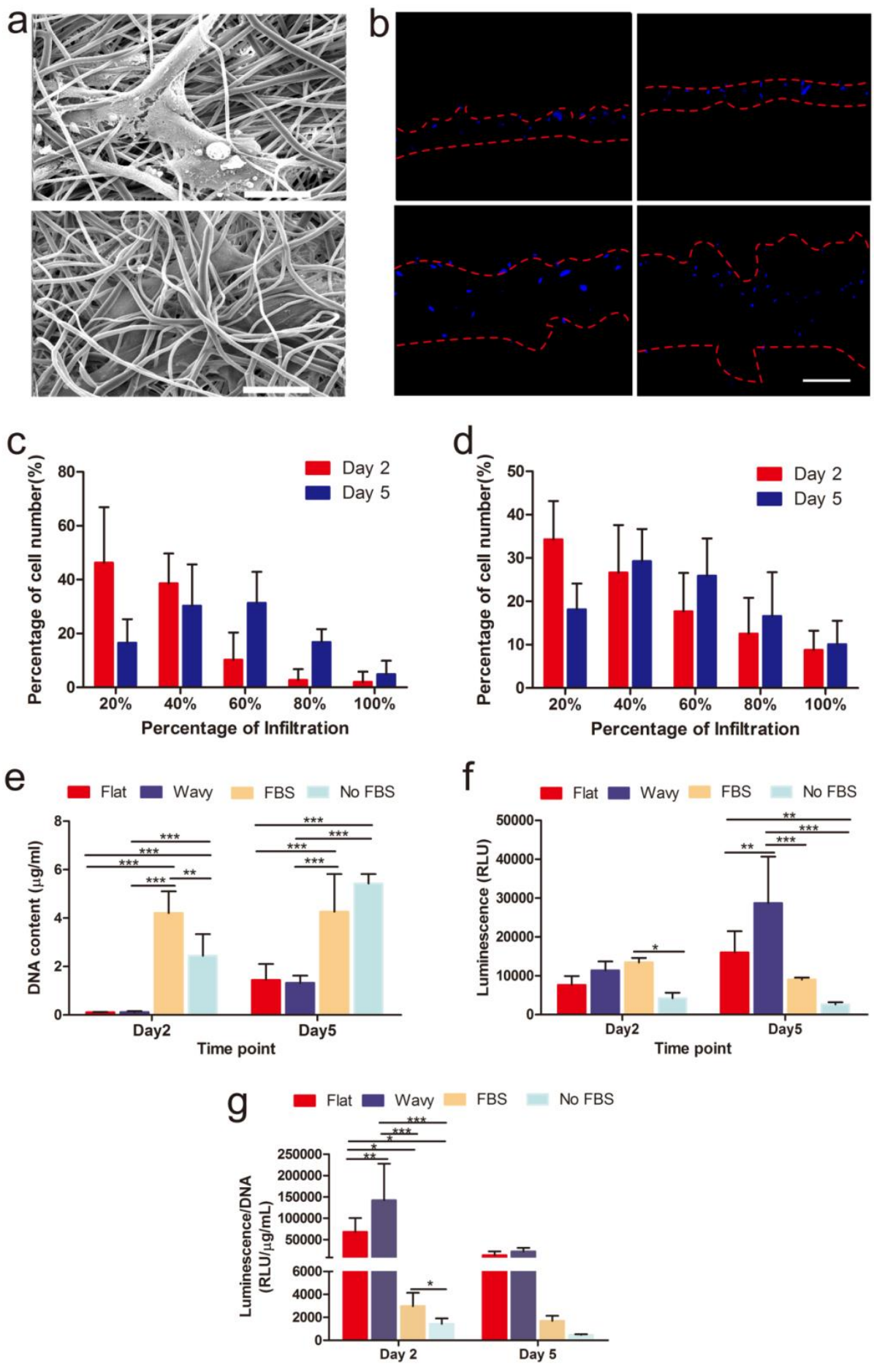

Figure 5. Cell penetration and Luciferase assay. (a) The morphology of hMSCs grown on flat (top) and wavy (bottom) scaffolds. (b) The scaffold cross-section showing cell nuclei labeled with DAPI. (c) and (d) The distribution of cells present in each depth section of the flat and wavy scaffold. For each scaffold region, the number of cells was counted and normalized to the total cell number present in the sample. The cellular infiltration depth is normalized to the thickness of scaffolds. (e) Quantification of DNA amount. (f) Comparison of Luciferase expression between flat and wavy scaffolds. (g) Luciferase expression with DNA normalization. Scale bars: $10 \mu \mathrm{m}$ (a) and $200 \mu \mathrm{m}$ (b). 


\subsubsection{TGF- $\beta$ signaling}

Given the resemblance of the obtained fibers with wavy patterns of connective tissues, we investigated if the wavy patterns could modulate the TGF- $\beta$ pathway, a key signaling pathway involved in connective tissue development. A mink lung epithelial cell line harboring a TGF- $\beta$ reporter construct (T-MLEC) was used to that end [29]. An increase in luciferase activity corresponds to an increase in TGF- $\beta$ signaling. In the present experiments, fetal bovine serum (FBS) that does contain TGF- $\beta$ was used as a positive control [30]. As shown in Figure $5 f$, cells seeded on a wavy scaffold emitted significant more light than cells seeded on a flat scaffold at day 5 , suggesting a corresponding increase in TGF- $\beta$ signaling.

\subsubsection{Similarities between native tissues and the scaffolds fabricated}

Figure S10 shows a few examples of curled pattern characteristics of connective tissues that could be recreated with the scaffolds proposed here. The organization of musculoskeletal tissues (Figure S10a), connective eye tissue like the iris (Figure S10c), and the artery wall (Figure S10e) composed of curled collagen fibers interconnected by a matrix can be mimicked by several of the curly fibers and wavy meshes that we can fabricate with this technique (Figure $S 10 b, d$ and $f$ ). The trachea also displays a crimped pattern (Figure $\mathrm{S10g}$ ) that could be mimicked by a wavy high-density scaffold that was electrospun for 45 min with the fibers lying down perpendicular to the shrinkage axis (Figure S10h).

\subsection{Conclusions}

In summary, we have demonstrated a simple but effective technique for generating crimped patterns on electrospun fibers by exploiting thermo-shrinkable materials. The wavy pattern features could be tuned by adjusting processing parameters, including the orientation of the deposited fibers, the percentage of shrinkage of the thermo-shrinking films and the use of sacrificial fibers. By controlling the spinning time, we were able to transfer the curls from a single fiber to multiscale waves in a fiber mesh. This approach opens up many opportunities in generating wavy patterns, for example gradient curls, and wavy patterns in a tubular geometry. In addition, the proposed technique can be readily extended to many other polymer fibers and thermos-shrinkable materials to generate functional scaffolds that could be beneficial for mimicking a variety of tissues organizations, such as iris, trachea, muscles, artery wall, and ciliary body. Furthermore, 
From From Fiber Curls to Mesh Waves: a Platform for the Fabrication of Hierarchically Structured Electrospun Fibers Mimicking Natural Tissue Formation

this new method may also inspire innovation in other application fields in future, such as functional textiles. 


\section{References}

[1] J.M. Caves, V.A. Kumar, W. Xu, N. Naik, M.G. Allen, E.L. Chaikof, Microcrimped Collagen Fiber-Elastin Composites, Advanced Materials 22(18) (2010) 2041-2044.

[2] A. Hiltner, J. Cassidy, E. Baer, Mechanical properties of biological polymers, Annual Review of Materials Science 15(1) (1985) 455-482.

[3] J. Diamant, A. Keller, E. Baer, M. Litt, R. Arridge, Collagen; ultrastructure and its relation to mechanical properties as a function of ageing, Proceedings of the Royal Society of London B: Biological Sciences 180(1060) (1972) 293-315.

[4] B.J. Rigby, N. Hirai, J.D. Spikes, H. Eyring, The mechanical properties of rat tail tendon, The Journal of General Physiology 43(2) (1959) 265-283.

[5] W. Liu, J. Lipner, C.H. Moran, L. Feng, X. Li, S. Thomopoulos, Y. Xia, Generation of Electrospun Nanofibers with Controllable Degrees of Crimping Through a Simple, Plasticizer-Based Treatment, Advanced Materials 27(16) (2015) 2583-2588.

[6] H. Chen, J. Huang, J. Yu, S. Liu, P. Gu, Electrospun chitosan-graft-poly ( $\varepsilon$-caprolactone)/poly ( $\varepsilon$ caprolactone) cationic nanofibrous mats as potential scaffolds for skin tissue engineering, International journal of biological macromolecules 48(1) (2011) 13-19.

[7] H. Chen, X. Fan, J. Xia, P. Chen, X. Zhou, J. Huang, J. Yu, P. Gu, Electrospun chitosan-graft-poly ( $\varepsilon-$ caprolactone)/poly ( $\varepsilon$-caprolactone) nanofibrous scaffolds for retinal tissue engineering, International journal of nanomedicine 6 (2011) 453.

[8] B. Sun, Y. Long, H. Zhang, M. Li, J. Duvail, X. Jiang, H. Yin, Advances in three-dimensional nanofibrous macrostructures via electrospinning, Progress in Polymer Science 39(5) (2014) 862-890.

[9] A. Hasan, A. Memic, N. Annabi, M. Hossain, A. Paul, M.R. Dokmeci, F. Dehghani, A. Khademhosseini, Electrospun scaffolds for tissue engineering of vascular grafts, Acta biomaterialia 10(1) (2014) 11-25.

[10] G. Cadafalch Gazquez, H. Chen, S.A. Veldhuis, A. Solmaz, C. Mota, B.A. Boukamp, C.A. van Blitterswijk, J.E. ten Elshof, L. Moroni, Flexible Yttrium-Stabilized Zirconia Nanofibers Offer Bioactive Cues for Osteogenic Differentiation of Human Mesenchymal Stromal Cells, ACS nano (2016).

[11] D.C. Surrao, J.W. Hayami, S.D. Waldman, B.G. Amsden, Self-crimping, biodegradable, electrospun polymer microfibers, Biomacromolecules 11(12) (2010) 3624-3629.

[12] D.C. Surrao, J.C. Fan, S.D. Waldman, B.G. Amsden, A crimp-like microarchitecture improves tissue production in fibrous ligament scaffolds in response to mechanical stimuli, Acta biomaterialia 8(10) (2012) 3704-3713.

[13] A. Varesano, A. Montarsolo, C. Tonin, Crimped polymer nanofibres by air-driven electrospinning, European polymer journal 43(7) (2007) 2792-2798.

[14] P.N. Georgelos, J.E. Pelkie, D.L. Wilhoit, Biaxially oriented heat-shrinkable film, Google Patents, 1995.

[15] G. Beumer, C. Van Blitterswijk, D. Bakker, M. Ponec, Cell-seeding and in vitro biocompatibility evaluation of polymeric matrices of PEO/PBT copolymers and PLLA, Biomaterials 14(8) (1993) 598-604.

[16] G. Beumer, C. Van Blitterswijk, M. Ponec, Biocompatibility of a biodegradable matrix used as a skin substitute: an in vivo evaluation, Journal of biomedical materials research 28(5) (1994) 545-552.

[17] T. Han, D.H. Reneker, A.L. Yarin, Buckling of jets in electrospinning, Polymer 48(20) (2007) 6064-6076. 
[18] G. Chang, J. Shen, Helical nanoribbons fabricated by electrospinning, Macromolecular Materials and Engineering 296(12) (2011) 1071-1074.

[19] M. Guvendiren, J.A. Burdick, The control of stem cell morphology and differentiation by hydrogel surface wrinkles, Biomaterials 31(25) (2010) 6511-6518.

[20] S.A. Ruiz, C.S. Chen, Emergence of patterned stem cell differentiation within multicellular structures, Stem cells 26(11) (2008) 2921-2927.

[21] B.M. Baker, A.O. Gee, R.B. Metter, A.S. Nathan, R.A. Marklein, J.A. Burdick, R.L. Mauck, The potential to improve cell infiltration in composite fiber-aligned electrospun scaffolds by the selective removal of sacrificial fibers, Biomaterials 29(15) (2008) 2348-2358.

[22] A. Seidi, M. Ramalingam, I. Elloumi-Hannachi, S. Ostrovidov, A. Khademhosseini, Gradient biomaterials for soft-to-hard interface tissue engineering, Acta Biomater. 7(4) (2011) 1441-1451.

[23] A. Di Luca, B. Ostrowska, I. Lorenzo-Moldero, A. Lepedda, W. Swieszkowski, C. Van Blitterswijk, L. Moroni, Gradients in pore size enhance the osteogenic differentiation of human mesenchymal stromal cells in three-dimensional scaffolds, Scientific reports 6 (2016).

[24] J. Lipner, W. Liu, Y. Liu, J. Boyle, G. Genin, Y. Xia, S. Thomopoulos, The mechanics of PLGA nanofiber scaffolds with biomimetic gradients in mineral for tendon-to-bone repair, Journal of the mechanical behavior of biomedical materials 40 (2014) 59-68.

[25] N.F.A. Manan, S.N.A.M. Noor, N.N. Azmi, J. Mahmud, Numerical investigation of Ogden and MooneyRivlin material parameters, ARPN Journal of Engineering and Applied Sciences 10(15) (2015) 6329-6335.

[26] J. Rnjak-Kovacina, A.S. Weiss, Increasing the pore size of electrospun scaffolds, Tissue Engineering Part B: Reviews 17(5) (2011) 365-372.

[27] B.A. Blakeney, A. Tambralli, J.M. Anderson, A. Andukuri, D.-J. Lim, D.R. Dean, H.-W. Jun, Cell infiltration and growth in a low density, uncompressed three-dimensional electrospun nanofibrous scaffold, Biomaterials 32(6) (2011) 1583-1590.

[28] A.K. Ekaputra, G.D. Prestwich, S.M. Cool, D.W. Hutmacher, Combining electrospun scaffolds with electrosprayed hydrogels leads to three-dimensional cellularization of hybrid constructs, Biomacromolecules 9(8) (2008) 2097-2103.

[29] M. Abe, J.G. Harpel, C.N. Metz, I. Nunes, D.J. Loskutoff, D.B. Rifkin, An assay for transforming growth factor-beta using cells transfected with a plasminogen activator inhibitor-1 promoter-luciferase construct, Analytical biochemistry 216(2) (1994) 276-84.

[30] T. Oida, H.L. Weiner, Depletion of TGF- $\beta$ from fetal bovine serum, J. Immunol. Methods 362(1) (2010) 195-198. 


\section{Supporting Information}
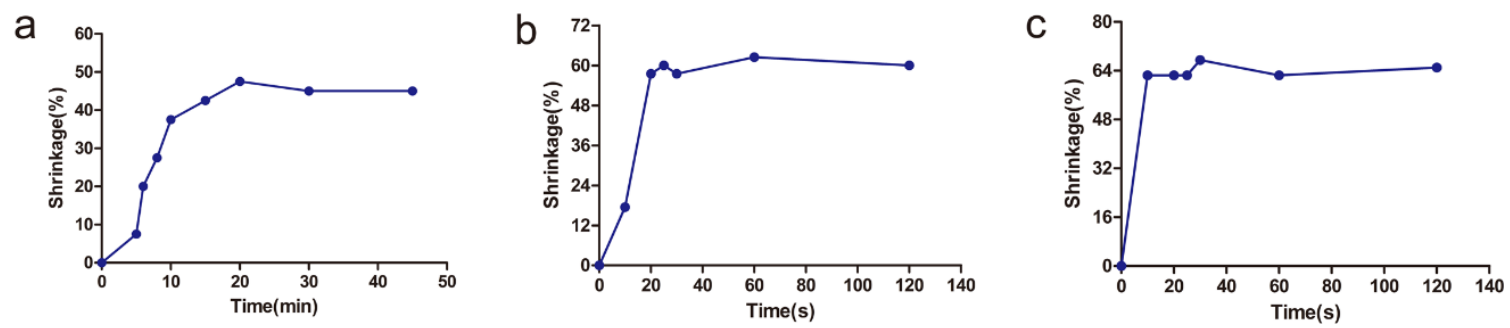

Figure S1. Shrinking properties of a monoaxially oriented PLA film at different temperatures over different times. (a) $65 \stackrel{\circ}{\circ}$, (b) $75 \stackrel{\circ}{ } \mathrm{C}$ and (c) $85 \stackrel{\circ}{\circ}$.
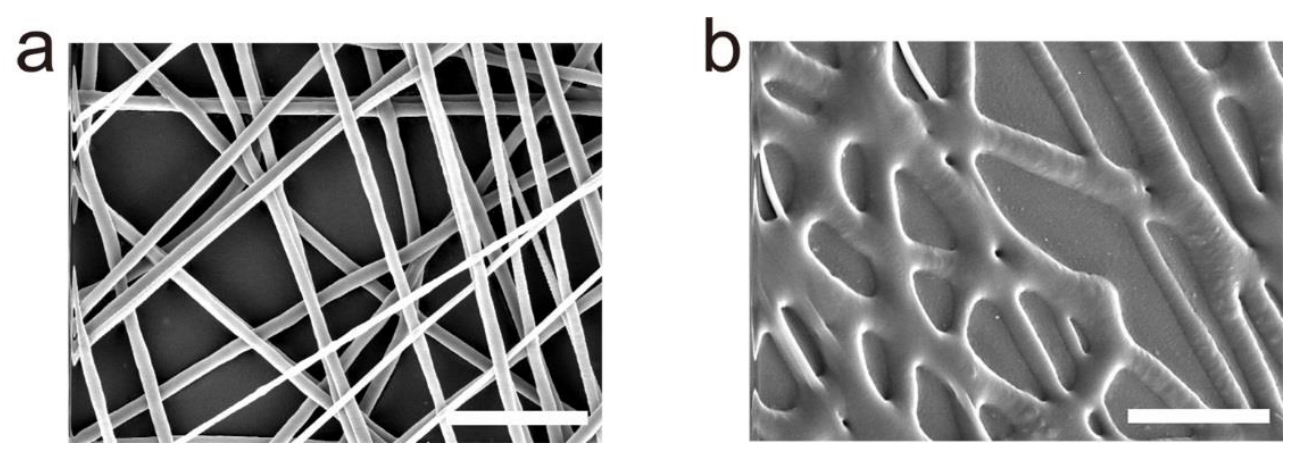

Figure S2. Failed buckling PCL fibers with oriented PLA films. (a) PCL fibers before shrinking. (b) PCL fibers after shrinking. PCL fibers become molten and no crimped pattern could be observed. This was because the melting point of PCL is lower than the shrinking temperature of PLA. Scale bar: $20 \mu \mathrm{m}$ ( $\mathrm{a}$ and b). 

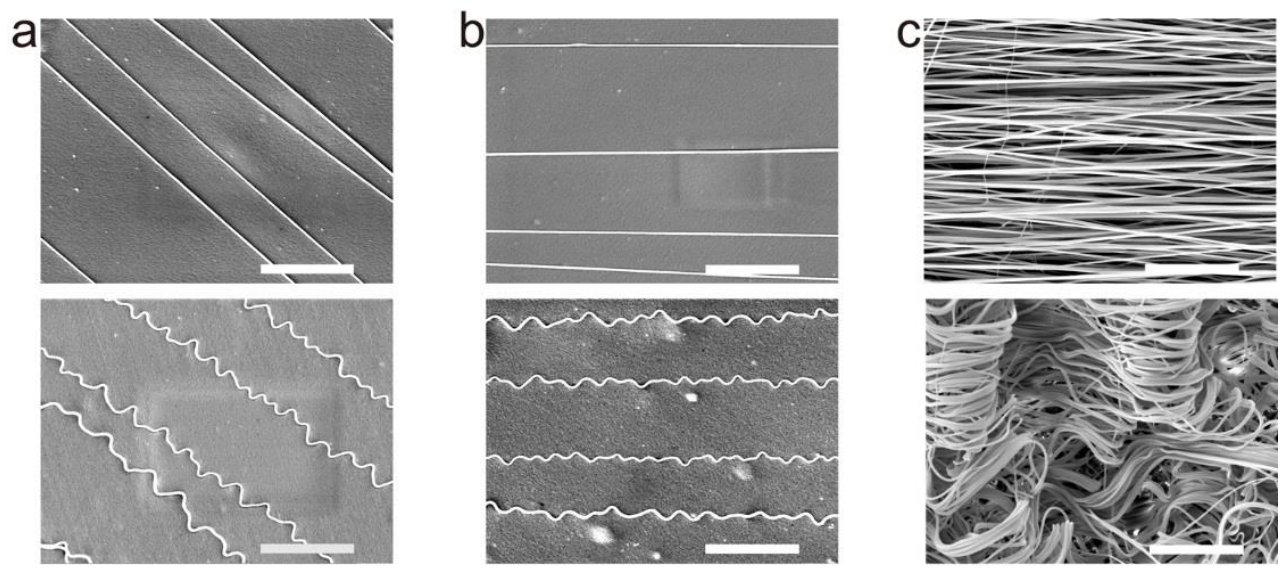

Figure S3. Fabricating curled PVA fibers using oriented PLA films. (a) Single fiber with alignment diagonal to the shrinking direction of PLA film before (top) and after (bottom) shrinking. (b) Fibers with alignment parallel to shrinking direction of PLA films before (top) and after (bottom) shrinking. (c) Fiber mesh with alignment parallel to the shrinking direction of the PLA film before (top) and after (bottom) shrinking. Scale bar: $20 \mu \mathrm{m}(\mathrm{a}-\mathrm{c})$.
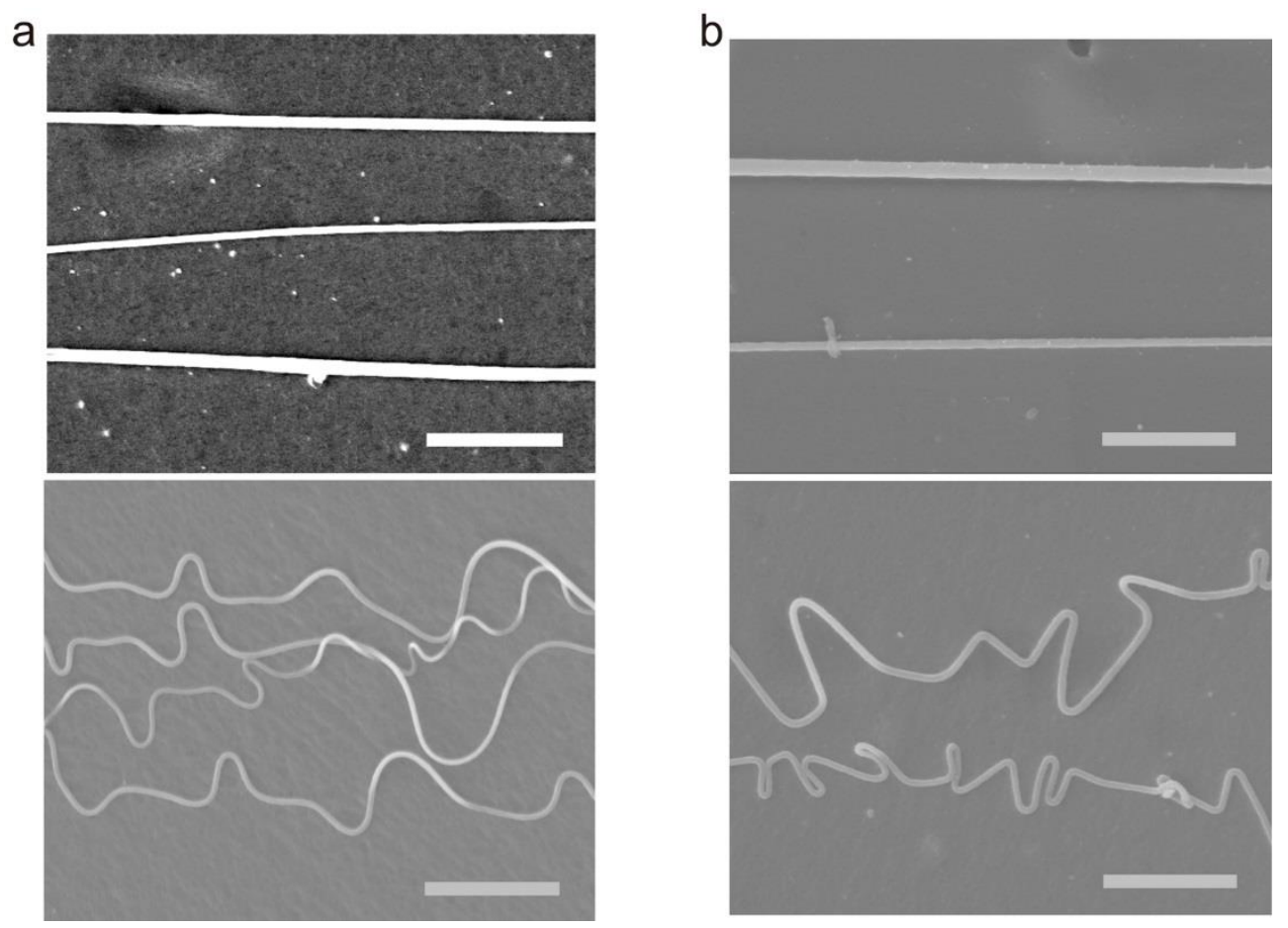

Figure S4. Fabrication of PAN and PS fibers using oriented PLA films. (a) PAN fibers deposited on PLA film before (top) and after (bottom) shrinking. (b) PS fibers deposited on PLA film before (top) and after (bottom) shrinking. Scale bar: $10 \mu \mathrm{m}(\mathrm{a}$ and $\mathrm{b})$. 

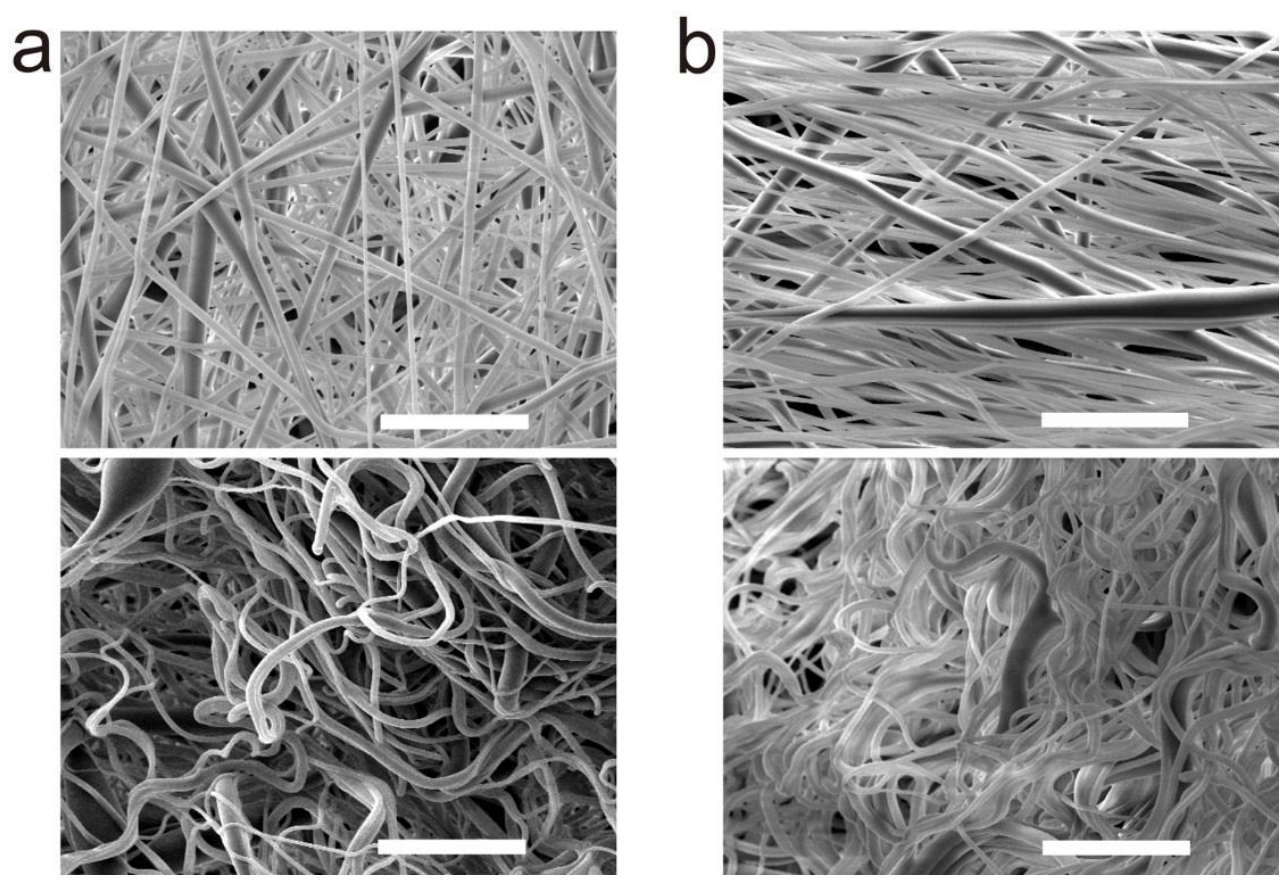

Figure S5. Fabricating curled PEOT/PBT fibers using oriented PS films. (a) Random PEOT/PBT fibers before (top) and after (bottom) shrinking. (b) Aligned PEOT/PBT fibers before (top) and after (bottom) shrinking. Scale bar: $20 \mu \mathrm{m}(a-b)$.
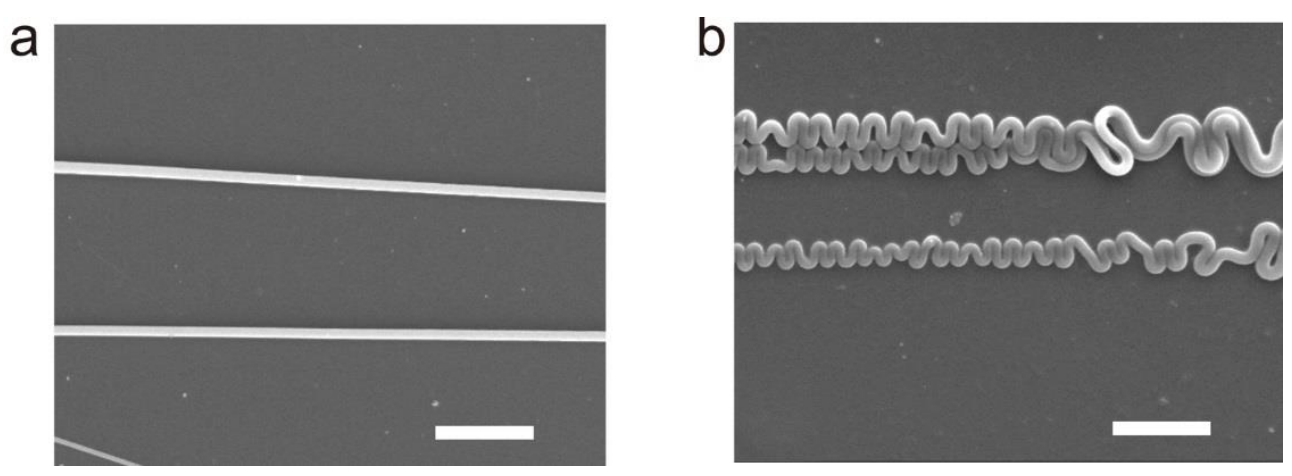

Figure S6. Fabricating curled PAN fibers using oriented PS films. (a) PAN fibers before shrinking. (b) PAN fibers after shrinking. Scale bar: $10 \mu \mathrm{m}$ ( $a$ and $b)$. 

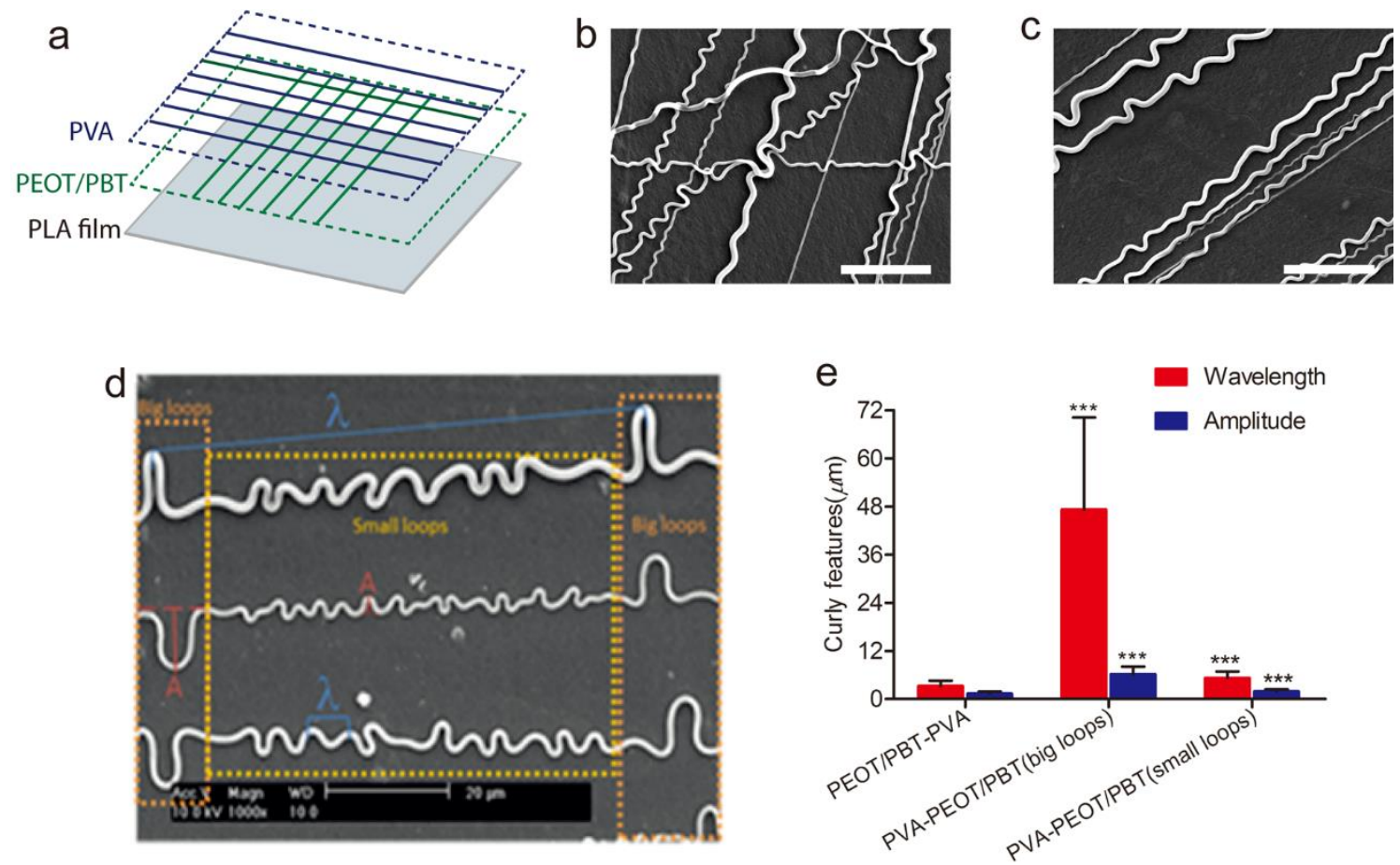

Figure S7. (a) Illustration of fabricating PEOT/PBT-PVA fibers. PEOT/PBT fibers were firstly deposited on the surface of the PLA film, then a second layer of PVA fibers was deposited on top of it. (b) SEM image of curled PEOT/PBT-PVA fibers before sacrifying the PVA fibers. (c) The morphology of curled PEOT/PBTPVA fibers after sacrifying the PVA fibers. (d) Figure depicting the ways to measure wavelengths and amplitudes for big loops and small loops. (e) Comparison of curly features between PEOT/PBT-PVA and PVA-PEOT/PBT fibers. PEOT/PBT-PVA corresponds to PEOT/PBT as first layer of deposition and PVA as second layer, and PVA-PEOT/PBT corresponds to PVA as first layer and PA as second layer. The distinction between big loops and small loops is explained in (d), where the same fiber has two different curls that were measured separately. ${ }^{* * *} p$-value $<0.001$. Scale bar: $20 \mu \mathrm{m}$ (b and $\left.c\right)$.
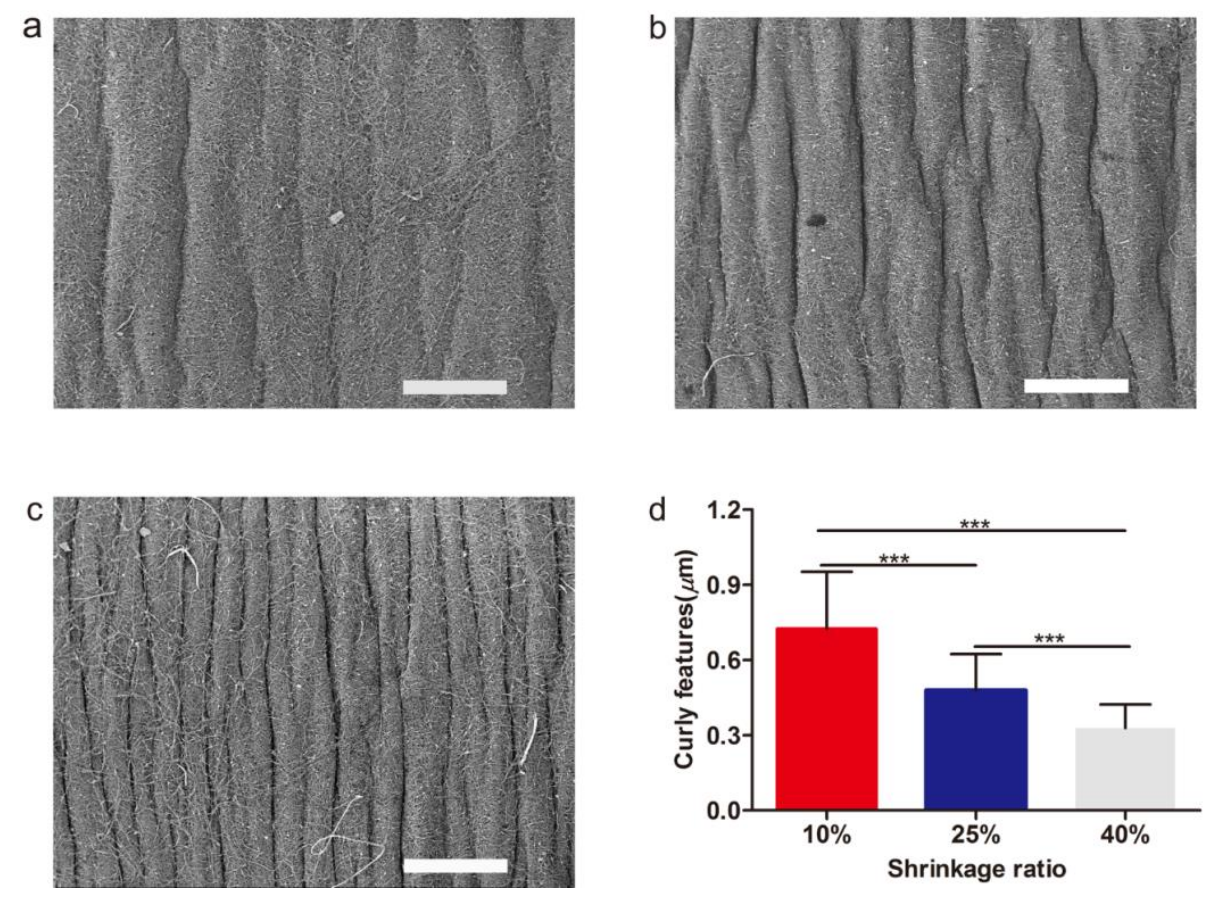
Figure S8. The influence of shrinkage ratio on wavy feature. (a) The morphology of wavy pattern at $10 \%$ shrinkage ratio. (b) The morphology of wavy pattern at $25 \%$ shrinkage ratio. (c) The morphology of wavy pattern at $40 \%$ shrinkage ratio. (d) Quantification of curly features generated from different shrinkage ratios. ${ }^{* * *}$-value $<0.001$. Scale bar: $1 \mathrm{~mm}(\mathrm{a}-\mathrm{c})$.

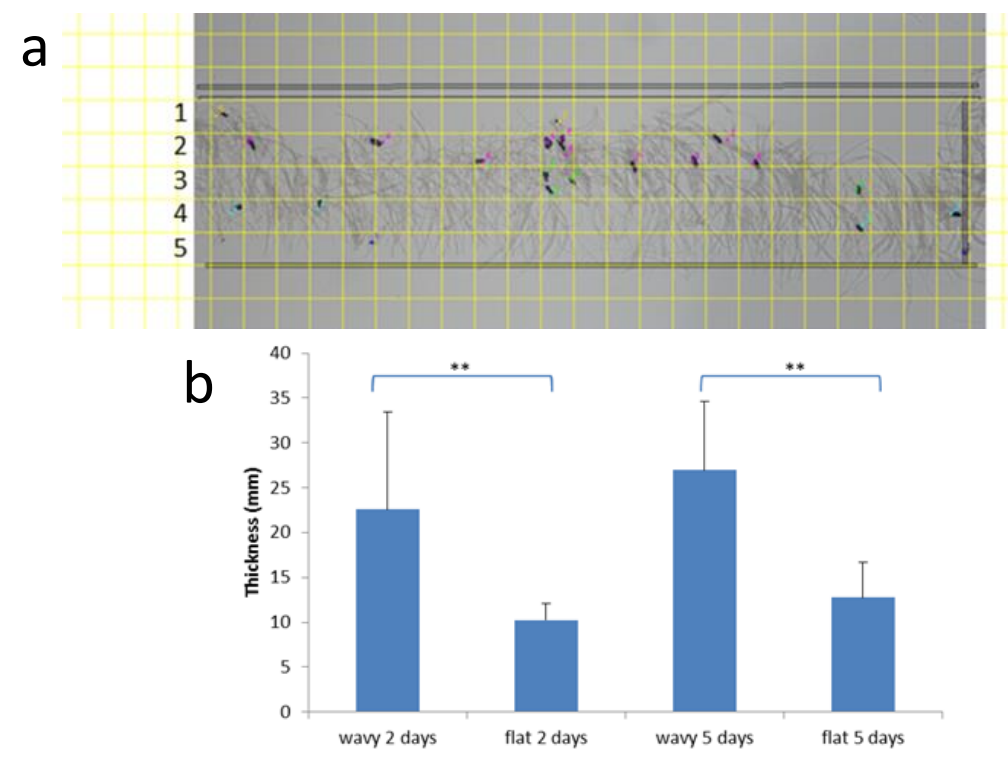

Figure S9. (a) Grid created in ImageJ to measure cell infiltration along the cross-section. The numbers represent the bins for each scaffold cross-section, 1 corresponding to the top and 5 to the bottom; (b) The graph shows the doubling effect in thickness registered after shrinkage. ${ }^{* *} p$-value $<0.05$. 

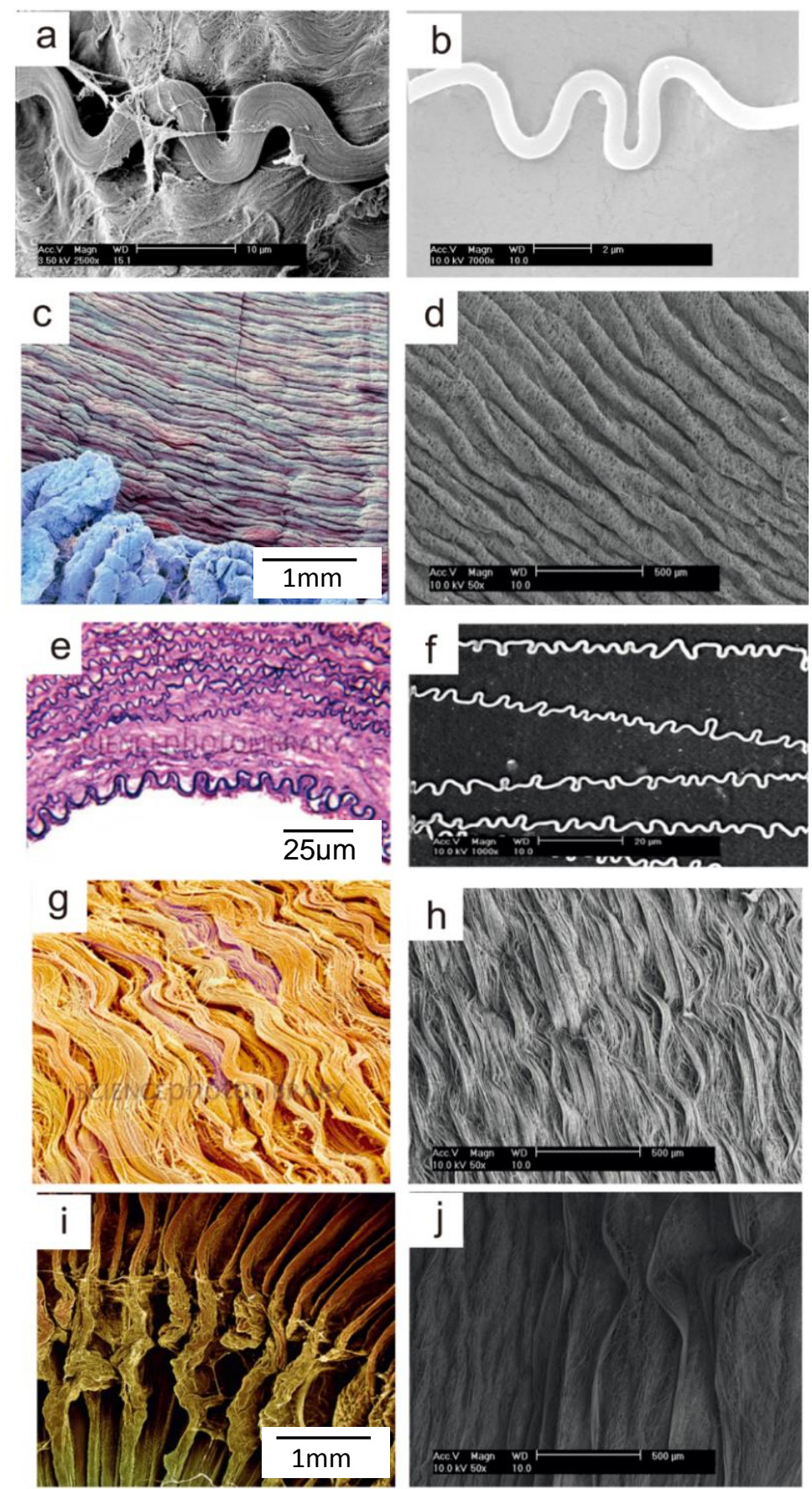

Figure S10. Similarities between native tissues and the fibers obtained. (a) A SEM image of musculoskeletal tissue composed wavy fibers. (b) Fibers fabricated in the present study exhibit a similar wavy configuration to a. (c) A SEM image of the iris (scale bar: $1 \mathrm{~mm}$; from www.sciencephoto.com). (d) The features of the scaffold in the present study show a similarity to that of the iris in c. (e) An image of the artery wall composed of curled elastin bundles separated by collagen (from www.sciencephoto.com). (f) A shrunk low density scaffold in the present study resembles the structure in e. (g) A SEM image of a tracheal wall displaying a crimp pattern (from www.sciencephoto.com), the scale bar is unknown. (h) High density shrunk scaffold in the present study resemble g. (i) A SEM image of a ciliary body. (j) The structure obtained in the present study having similarity to $\mathrm{i}$. 
Table S1. Mechanical analysis.

\begin{tabular}{|c|c|c|c|c|c|}
\hline Shrinkage & $\begin{array}{c}\text { Young's } \\
\text { modulus } \\
(\mathrm{MPa})\end{array}$ & $\begin{array}{c}\text { Linearity } \\
\text { range } \\
(\%)\end{array}$ & $\begin{array}{c}\text { Ultimate } \\
\text { stress (MPa) }\end{array}$ & $\begin{array}{c}\text { Ultimate } \\
\text { strain } \\
(\%)\end{array}$ & $\begin{array}{c}\text { Strain energy } \\
\text { density } \\
\text { (MPa) }\end{array}$ \\
\hline Parallel & $9.91 \pm 2.24$ & $1-7$ & $2.75 \pm 0.76$ & $131.78 \pm 29.42$ & $3.04 \pm 0.87$ \\
\hline $10 \%$ & $5.12 \pm 1.15$ & $1.8-10$ & $2.24 \pm 0.33$ & $245.96 \pm 21.37$ & $4.66 \pm 0.77$ \\
\hline $25 \%$ & $6.49 \pm 1.64$ & $1.8-10$ & $2.69 \pm 0.47$ & $199.18 \pm 37.93$ & $5.28 \pm 1.67$ \\
\hline $30 \%$ & $4.61 \pm 1.16$ & $1-7$ & $1.24 \pm 0.20$ & $112.64 \pm 37.74$ & $1.27 \pm 0.50$ \\
\hline $40 \%$ & $2.87 \pm 0.46$ & $1-9$ & $1.37 \pm 0.32$ & $150 \pm 32.56$ & $1.76 \pm 0.53$ \\
\hline $50 \%$ & $2.40 \pm 0.31$ & $1-12$ & $1.13 \pm 0.15$ & $124.9 \pm 26.64$ & $1.19 \pm 0.25$ \\
\hline
\end{tabular}

\section{Supplementary Note: Mechanical modeling}

The results of the model fitting are summarized in Table S2.

Table S2. Mooney-Rivlin model: parameters estimation.

\begin{tabular}{l|lll|l}
\hline Shrinkage & C01 & C10 & C20 & $\mathbf{R}^{\mathbf{2}}$ \\
\hline Parallel & $3.39 \pm 1.33$ & $-1.43 \pm 0.89$ & $0.130 \pm 0.120$ & $0.962 \pm 0.011$ \\
$\mathbf{1 0 \%}$ & $1.29 \pm 0.24$ & $-0.17 \pm 0.08$ & $0.007 \pm 0.003$ & $0.974 \pm 0.007$ \\
$\mathbf{2 5 \%}$ & $2.06 \pm 0.54$ & $-0.47 \pm 0.25$ & $0.022 \pm 0.011$ & $0.972 \pm 0.003$ \\
$\mathbf{3 0 \%}$ & $1.93 \pm 0.79$ & $-0.91 \pm 0.57$ & $0.081 \pm 0.062$ & $0.986 \pm 0.003$ \\
$\mathbf{4 0 \%}$ & $1.28 \pm 0.41$ & $-0.44 \pm 0.27$ & $0.032 \pm 0.024$ & $0.961 \pm 0.011$ \\
$\mathbf{5 0 \%}$ & $1.13 \pm 0.50$ & $-0.37 \pm 0.34$ & $0.032 \pm 0.041$ & $0.982 \pm 0.018$ \\
\hline
\end{tabular}


A statistically significant decrease of the parameter $\mathrm{C}_{01}$ with the increase of the shrink percentage was detected. A similar trend was found for the parameter $\mathrm{C}_{10}$. No statistical differences were found among the different percentages of shrinkage for the parameter $\mathrm{C}_{20}$.
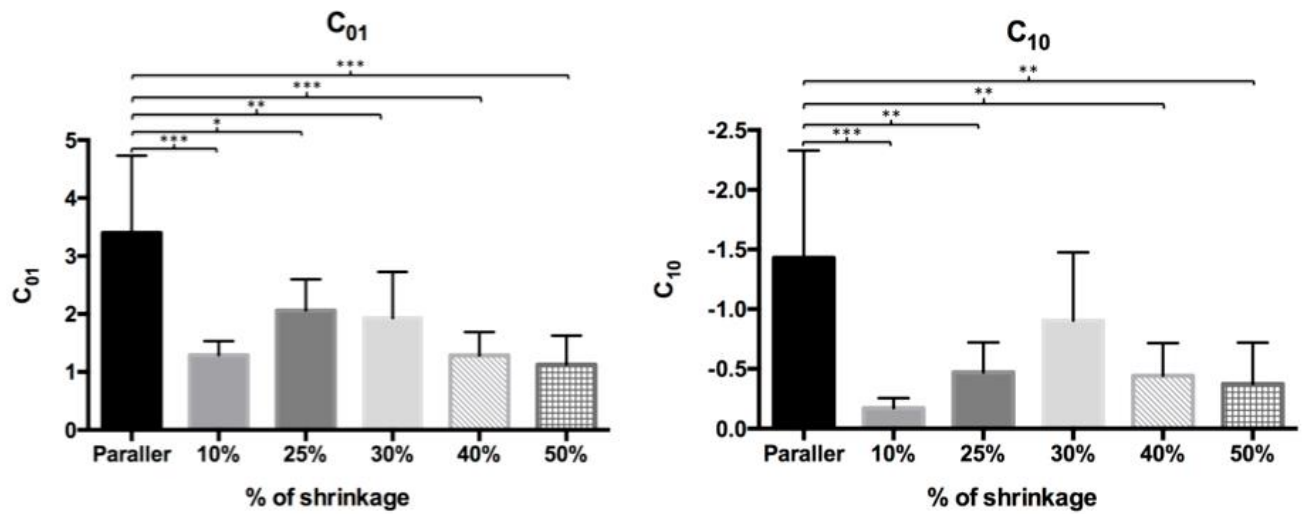

$\mathrm{C}_{20}$

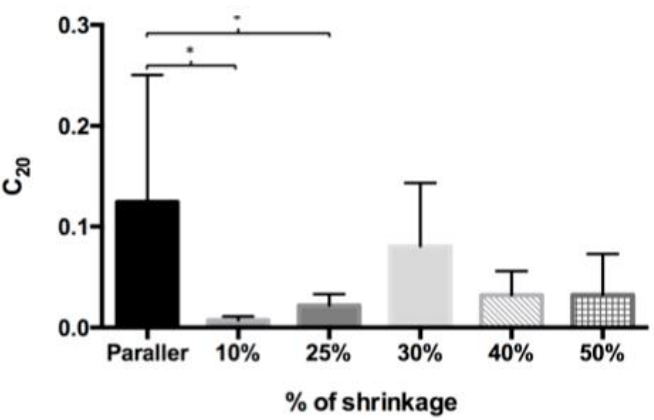

Figure S11. Mooney-Rivlin model: comparisons of different conditions for each parameter. 


\section{Chapter 6}

\section{Direct Writing Electrospinning of Scaffolds with Multi- dimensional Fiber Architecture for Hierarchical Tissue Engineering}

Honglin Chen, Afonso Malheiro, Clemens A. Van Blitterswijk, Carlos Mota, Paul Wieringa, Lorenzo Moroni

Department of Complex Tissue Regeneration

MERLN Institute for Technology-Inspired Regenerative Medicine

Maastricht University

6200 MD Maastricht, The Netherlands 


\begin{abstract}
Nanofibrous structures have long been used as scaffolds for tissue engineering (TE) applications, due to their favorable characteristics such as high porosity, flexibility, high cell attachment and enhanced proliferation, and overall resemblance to native extracellular matrix (ECM). Such scaffolds can be easily produced at a low cost via electrospinning (ESP), but generally cannot be fabricated with a regular and/or complex geometry, characterized by macropores and uniform thickness. We present here a novel technique for direct-writing (DW) with ESP to produce complex three-dimensional (3D) multi-scale and ultrathin $(\sim 1 \mu \mathrm{m})$ fibrous scaffolds with regular pore sizes and controllable fiber bundle morphology. Using this technique, a scaffold mimicking the zonal organization of articular cartilage was fabricated as a proof of concept, demonstrating the ability to better mimic native tissue organization. The DW scaffolds directed tissue organization and fibril matrix orientation in a zone-dependent way. Comparative expression of chondrogenic markers revealed a substantial upregulation of Sox 9 and aggrecan (ACAN) on these structures compared to conventional electrospun meshes. Our novel method provides a simple way to produce customized 3D ultrathin fibrous scaffolds, with great potential for TE applications, in particular those for which anisotropy is of importance.
\end{abstract}

\title{
Keywords:
}

Direct-writing, electrospinning, tissue engineering, articular cartilage, human mesenchymal stromal cells 


\subsection{Introduction}

Articular cartilage is present in joints to provide a smooth, lubricated surface for articulation and resistance to high dynamic compressive loads[1]. Histologically, it is characterized by sparse chondrocytes distributed within a dense extracellular matrix (ECM), mainly composed of water, proteoglycans, glycoproteins, and collagen in a zonal configuration. These layers are distinguished as superficial, middle, deep, and calcified zone, which vary in molecular composition, cell density, and collagen orientation[2]. Articular cartilage is devoid of mesenchymal progenitors and blood vessels, and as a result, the potential for self-repair is limited. Therefore, when damage occurs, there is a chance for progressive degeneration that may lead to osteoarthritis[3].

Tissue engineering (TE) of cartilage constructs is a promising approach to replace the currently suboptimal clinical treatments, aiming to develop a biomimetic replacement with appropriate mechanical behaviour. Additive manufacturing (AM) techniques have greatly contributed to this goal. These technologies have provided valuable tools for the custom fabrication of 3D scaffolds with reproducible patterns, tunable porosity, and tailored physicochemical properties[4]. However, limitations inherent to these processes still persist. Notably, the required setups are typically expensive and the range of usable materials is dependent on the fabrication technique, for instance extrudable thermoplastic polymers for fused deposition modelling. These techniques also introduce other concerns such as thermal degradation of the polymer during the fabrication process[4]. Moreover, current $A M$ techniques possess low spatial resolution and thus the features of the produced scaffolds are typically several orders of magnitude larger than ECM fibers, particularly collagen fibrils. This results in a poor cell-seeding efficiency and proliferation, as well as non-uniform scaffold coverage.

Nanofibrous scaffolds are interesting alternatives for cartilage TE, with a highly porous structure reminiscent of the ECM milieu that has been shown to promote cell attachment, and stimulate cell function while facilitating the efficient exchange of nutrients, oxygen, and metabolites[5]. Nanofibers are also very flexible due to their high aspect ratio, allowing cells to remodel their surroundings with produced matrix[6]. To fabricate nanofibrous mats, solution electrospinning (ESP) is one of the most popular methods, since it is easy to operate, relatively inexpensive, and versatile in terms of materials selection[7]. ESP is a complex hydrodynamic process governed by many variables and characterized by an unpredictable electrified jet[8]. The chaotic nature of this jet results from the build-up of surface charges generated by an applied electrostatic force, which 
initially leads to droplet elongation, Taylor cone formation, and jet emanation, but soon overcomes the liquid surface tension, causing bending instabilities[8, 9]. As charge density increases, repulsion forces alter the stable jet into a whipping jet. At this point, fiber deposition occurs randomly and thus accurate pattern designing is impeded. Therefore, most ESP products are dense meshes that lack a regular pore-size network and have an architecture defined by the collector shape (e.g., flat or grooved plate, rotating drums, spaced electrodes, etc.) [9, 10].

To achieve control over an ESP process would enable the combined benefits of the topography provided by electrospun mats with the reproducibility and designing potential of AM techniques. Therefore, much interest has been taken in the pursuit of direct writing (DW) with ESP, with some efforts already reported. One strategy is based on the application of an electrostatic lens system to focus the electrical field into a narrow region. Through the use of a ring electrode positioned between the spinneret and collector, Neubert et al. and Bellan et al. showed the targeted deposition of fibers into a single spot $[11,12]$. By either moving the collector or using steering electrodes, simple lines could be patterned onto the collector, but no complex patterning was achieved, A similar but improved strategy was followed by Jongwan Lee et al., who employed a side-wall electrode, a thin glass plate collector and a sharp-pin electrode to focus the electrified jet and draw regular 3D nanofibrous patterns[13], which could later be stacked to build a larger scaffold[14]. Although successful, the aforementioned approach relied on a complex setup that is difficult to adopt in other labs. An earlier and simpler approach that dispenses additional setup modifications is near-field electrospinning (NFESP), in which the unstable region of the jet is bypassed, by closely approximating the spinneret to the collector and using very low voltages[15-17]. However, drawbacks to this technique are evident, as it relies on a solution-dipped probe for jet feeding, which greatly limits the possible scaffold size.

In another approach, melt ESP replaces the polymer solution with a molten polymer to generate fibers $[18,19]$. Application of melt ESP for DW of poly ( $\varepsilon$-caprolactone) (PCL) fibrous structures for hydrogel reinforcement has revealed great potential in engineered cartilage constructs[20]. This technique has been also used to fabricate cell-invasive 3D scaffolds constructed from photo-cross-linkable poly(L-lactide-co- $\varepsilon$-caprolactone-coacryloyl carbonate) microfibers[21, 22]. Because of the use of high melting temperatures, the technique may cause polymer degradation and is restricted to the use of 
thermoplastic polymers. In addition, it excludes the addition of bioactive molecules, such as collagen, which undergo denaturation at high temperatures[19, 23]. The minimum feature size is also a concern in melt ESP, since the Barus effect induces a jet enlargement[24] and obstructs formation of nanofibers, although recent efforts have shown the production of sub-micron fibers in special conditions[25]. This process also produces a single fiber at a time, while tissue development benefits from the presence of multiple interconnected fibers that act as anchoring points for cells to assemble focal adhesions[26].

Here, we show the development of a novel DW technique that modifies conventional solution ESP to produce accurately patterned ultrathin 3D scaffolds. We determined the optimal conditions for DW ESP, including solution, ambient conditions, and processing parameters, to focus the jet to a single point and collect the fibers in a dry state. Patterns could be designed via predefining $X Y$ translation of the collector, and the resulting fibers stacked to create 3D constructs. After characterizing this technological process, we fabricated scaffolds that mimic the zonal configuration of articular cartilage. These scaffolds were seeded with human mesenchymal stromal cells (hMSCs) and cultured in chondrogenic differentiation medium over the course of 21 days. The DW ESP scaffolds successfully supported hMSC proliferation and chondrogenic differentiation, as evaluated by matrix production and orientation, tissue development, and gene expression. These data argue that DW ESP can fabricate ultrathin scaffolds with multidimensional fiber architecture and favorable biological characteristics, which has great potential for wide TE applications.

\subsection{Results and Discussion}

Traditional ESP generally deposits fibers with random morphology due to the bending instability of the electrospinning jet (Figure $1 \mathrm{~A}$ ). However, controlled fiber deposition, and thus patterning, is possible by DW with ESP, and requires the jet to follow a stable path from the moment it emanates from the spinneret tip, until it reaches the collector (Figure 1B). To fabricate suitable scaffolds to mimic articular cartilage, we deployed DW with ESP. First, we optimized the ESP process for DW, including polymer solution, flow rate, applied voltage, working distance and ambient conditions.

\subsubsection{Solution conditions}


For these studies, we used a polymer solution of Poly(ethylene oxide terephthalate)/poly(butylene terephthalate)(PEOT/PBT) for the scaffolds, because PEOT/PBT has been demonstrated as a biocompatible and degradable polymer with promising application for skeletal regeneration[27, 28]. Elongation and stability of the ESP jet depends on optimal solution parameters. Solutions with a very high viscosity usually exhibit longer stress relaxation times, which can prevent fracturing of the jet during ESP[9]. We tested solution mixtures with polymer concentrations between $26-30 \%(\mathrm{w} / \mathrm{v})$ and $\mathrm{CHCl}_{3}: \mathrm{HFIP}$ solvent ratios between 70:30 and 80:20 (v/v). We found that the optimal range of polymer concentration that permits DW was $27-29 \%(\mathrm{w} / \mathrm{v})$, which yielded fairly viscous solutions. The optimal range of solvent ratio that permits $D W$ was between 74:26 and 78:22 (v/v), which ensured good polymer dissolution and yielded a solution with high evaporation rate. A solution with high evaporation rate is particularly important for the DW technique, since it enables fibers to dry quickly within shorter working distances $(\mathrm{Wd})$ used in these studies. Within the optimal range of polymer concentration and solvent ratio, the solution with $28 \%(\mathrm{w} / \mathrm{v})$ polymer in $75: 25 \mathrm{CHCl}_{3} / \mathrm{HFIP}(\mathrm{v} / \mathrm{v})$ revealed the best outcome for a stable and continuous DW process (Table S1).

\subsubsection{Solution flow rate}

The solution flow rate through the spinneret is an important factor, as it influences the amount of transferred material and jet velocity[9]. We tested flow rates from $0.05 \mathrm{~mL} / \mathrm{h}$ to $2 \mathrm{~mL} / \mathrm{h}$. We found that flow rates between 0.08 and $0.3 \mathrm{~mL} / \mathrm{h}$, a range that enables a low but steady flow of the polymer solution through the spinneret, were necessary for successful DW ESP. The influence of flow rates $(0.05,0.1$, and $0.5 \mathrm{~mL} / \mathrm{h})$ on the jet and fiber deposition is shown in Figure $\mathrm{S} 1 \mathrm{~A}-\mathrm{C}$ to exemplify the conditions tested. At a low flow rate of $0.05 \mathrm{~mL} / \mathrm{h}$, the amount of solution that was supplied through the spinneret was insufficient to maintain a regular jet for the applied voltage $(8 \mathrm{kV})$ and scan speed, thus leading to the deposition of an erratic line (Figure S1A). As the flow rate increased to $0.1 \mathrm{~mL} / \mathrm{h}$, a thin and steady jet was produced, resulting in the formation of straight line patterns (Figure S1B). When the polymer solution supply was $0.5 \mathrm{~mL} / \mathrm{h}$, the jet could no longer be focused and consequently, the result was the collection of dispersed fiber mesh (Figure S1C). Thus, we concluded that the optimal flow rate was $\sim 0.1 \mathrm{~mL} / \mathrm{h}$.

\subsubsection{Applied voltage and working distances}


In addition to the solution conditions and flow rate, the interplay between the applied voltage and the distance from the collector affects the solution jet. The applied voltage needs to reach a certain threshold to initiate the ESP process[8, 29]. As the voltage increases, the solution ejection also increases and higher electrostatic repulsive forces build up on the fluid jet[8, 9]. The optimal applied voltage is also dependent on the $\mathrm{Wd}$, as both parameters are related in the determination of the electric field strength[30]. A typical electrified jet will follow a short stable path before acquiring a whipping profile[31], thus selecting an appropriate $\mathrm{Wd}$ is of utmost importance. We tested applied voltages between 2-16 kV, with corresponding $W d$ values between 2-12 cm. The threshold voltages needed to initiate the ESP process increased as the Wd increased: the minimum voltages were $4 \mathrm{kV}$ at $2 \mathrm{~cm}, 4.5 \mathrm{kV}$ at $3 \mathrm{~cm}$ and $5.5 \mathrm{kV}$ at $4 \mathrm{~cm}$ (Table S2). It is worth mentioning that following jet initiation, the spinning voltage could be reduced to a value lower than its jet initiation voltage. For example, at $2 \mathrm{~cm}$, the lowest $\mathrm{Wd}$ tested, the minimum voltage for jet initiation was $4 \mathrm{kV}$ during DW ESP. However, following jet initiation, the spinning voltage could be reduced to a minimum value of $2.5 \mathrm{kV}$. At $7 \mathrm{kV}$ and a $\mathrm{Wd}$ of $3 \mathrm{~cm}$, we observed an unfocused jet, which broke down into multiple thin jets as a consequence of jet bending instability (Figure 1C). In this case, the amount of ejected solution provided insufficient viscoelastic force to balance surface charge density[32]. When we deceased the voltage to $5 \mathrm{kV}$, a stable jet was achieved during its flight time (Figure 1D), depositing and vertically building the fibers in a single point in the static collector. However, this process could not continue indeterminably due to an increase in charge accumulation, resulting in an increase of repulsive forces at that point (Figure S2). We then increased the $\mathrm{Wd}$ to $8 \mathrm{~cm}$ and tested different applied voltages. At 8 $\mathrm{kV}$, the jet trajectory was stable and straight lines could be fabricated (Figure S1D). At 12 $\mathrm{kV}$, the amount of ejected fluid increased, resulting in too much volume at the collector to produce single steady lines at the given scan speed (Figure S1E). At $16 \mathrm{kV}$, the repulsive charges accumulated and led to the onset of bending instabilities, resulting in the complete loss of the focusing effect (Figure S1F). When the Wd was further increased to $10 \mathrm{~cm}$ and $12 \mathrm{~cm}$, either the jet was collected in its whipping phase or the electric field strength was too low to focus it, resulting in unpredictable fiber deposition (Figure $\mathrm{S} 1 \mathrm{H}-\mathrm{I}$ ). From these data, we concluded that $\mathrm{Wd} \leq 8 \mathrm{~cm}$ and voltages $\leq 8 \mathrm{kV}$ were necessary to collect the jet in a stable and focusing manner.

From voltage/Wd parameters that resulted in successful focused spinning, we examined the morphology of the deposited fibers (Table S2) and found three different 
regimes (Figure 2D-F). The transition between these regimes depended both on $\mathrm{Wd}$ and electrical field strength (V), which could be simplified as the ratio V/Wd. Typically, for low $\mathrm{V} / \mathrm{Wd}$, the fibers were randomly deposited in a bundle and dispersed from each other (Figure 2D). As the $\mathrm{V} / \mathrm{Wd}$ ratio increased, fiber density increased and the distance between fibers decreased, resulting in increased fiber cohesiveness (Figure 2E). If the V/Wd ratio was increased by voltage, the fiber bundle displayed a more threedimensional core, with the fibers aligned in the direction of the jet movement, as well as a thicker fiber dimension (Figure 2A and Figure S3). If the V/Wd ratio was increased via reducing the $\mathrm{Wd}$, similar morphological changes was achieved together with an increase of fiber diameter (Figure 2B and Figure S4). The transition between a random deposition (lower limit) and an anisotropic alignment (upper limit), was defined by the V/Wd ratio, thus suggesting that these morphological changes are determined by the amount of solution ejected. To obtain a single fiber configuration (Figure $2 \mathrm{~F}$ ), a $\mathrm{V} / \mathrm{Wd}>2$ was required. For example, at a $\mathrm{Wd}$ of $2 \mathrm{~cm}$, an applied voltage of $4.5 \mathrm{kV}$ resulted in single fiber deposition. However, this was only possible at $\mathrm{Wd} \leq 2 \mathrm{~cm}$, since the threshold that defines the balancing of repulsive charges by the solution cohesive forces was also low.

\subsubsection{Scan speed}

The velocity at which the fibers were collected was also a parameter of great interest. If the voltage and $\mathrm{Wd}$ were maintained constant, the physical behaviour of the jet would be in principle the same. However, the velocity at which the jet or collector moves would influence the amount of fibers reaching a single point on the collector. Consequently, the amount of charges in a single spot can also vary and can determine the consistency of the fiber deposition. We maintained the voltage at $5 \mathrm{kV}$, Wd at $5 \mathrm{~cm}$, but varied the scan speed between 4 and $10 \mathrm{~mm} / \mathrm{s}$ to determine its effect on the deposited fibers (Figure S5). At a collection speed of $4 \mathrm{~mm} / \mathrm{s}$, the deposited layer was slightly incoherent with respect to its central axis and some fiber dispersion occurs. When the scan speed was increased to $7 \mathrm{~mm} / \mathrm{s}$, the accumulation of repulsive charges at a point decreased and the spun layer showed a more consistent pattern, but still presented some fiber dispersion from its core (Figure S5B). At a collection speed of $10 \mathrm{~mm} / \mathrm{s}$, the deposited layer showed little deviation from its central axis having comparatively less fiber dispersion (Figure S5C). In summary, as the collector scan speed increased, the line focusing was improved whereas line patterns assumed a proportionally shorter width (Figure 2C). From these data, we conclude that the optimal scan speed was $\sim 10 \mathrm{~mm} / \mathrm{s}$. 


\subsubsection{Ambient parameters}

Finally, the ambient parameters for ESP, such as temperature and relative humidity, were also important to tune. Because an inverse relationship exists between temperature and viscosity (crucial for jet elongation as mentioned above).[33] We reasoned that lower temperatures would facilitate jet focusing and relative higher temperatures may cause faster solvent evaporation, and consequent needle blocking by the polymer solution. Therefore, we tested temperatures between $18.5-30{ }^{\circ} \mathrm{C}$ and found the optimal temperature is $\sim 20^{\circ} \mathrm{C}$.

A

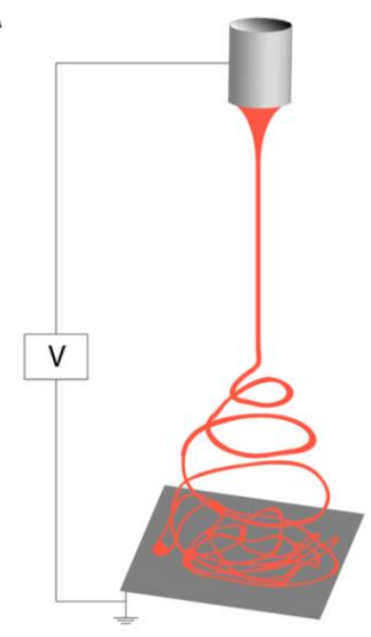

C

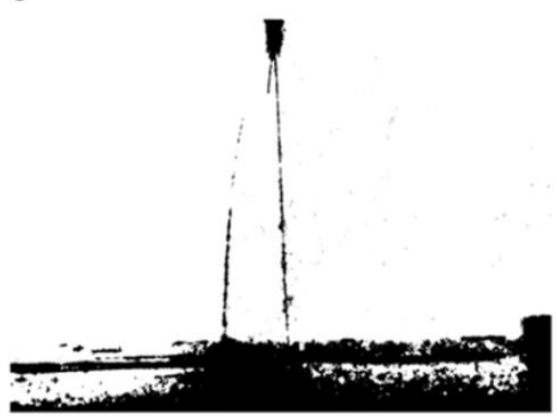

B

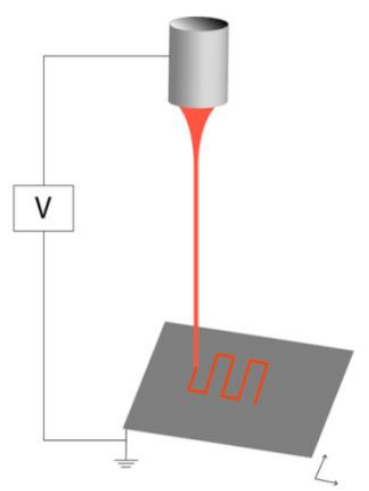

D

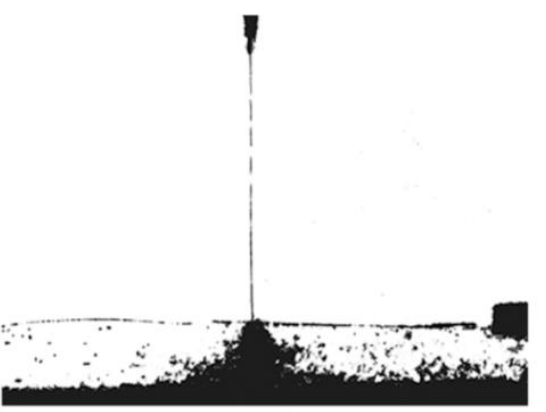

Figure 1. (A and B) Illustration of the ESP process in conventional $(A)$ and direct writing $(B)$ mode. In direct writing mode, the jet travels in a straight path and the translational stage moves in $X$ and $Y$ direction to create a pattern. ( $C$ and $D)$ Photos of electrified jet when travelling with an unfocused trajectory $(C ; V=7 \mathrm{kV}$ and $\mathrm{Wd}=3 \mathrm{~cm})$ and in stable path $(\mathrm{D} ; \mathrm{V}=5 \mathrm{kV}$ and $\mathrm{Wd}=3 \mathrm{~cm})$.

Relative humidity within the fairly large range of 25 to $55 \%$ allows for jet eruption and focusing, but we needed to optimize this parameter for long fabrication times. The production of large patterns requires the hemispherical drop at the spinneret tip to be maintained in a fluid state, which is facilitated by humidity levels. Furthermore, solutions 
containing volatile solvents may dry quickly in dry environments. High humidity also benefits fiber stacking by reducing the charge accumulation. It should be noted that excessive humidity results in melting the fiber morphology due to insufficient solvent evaporation. From the humidity tested, we found that the optimal relative humidity is $40 \pm$ $2 \%$.
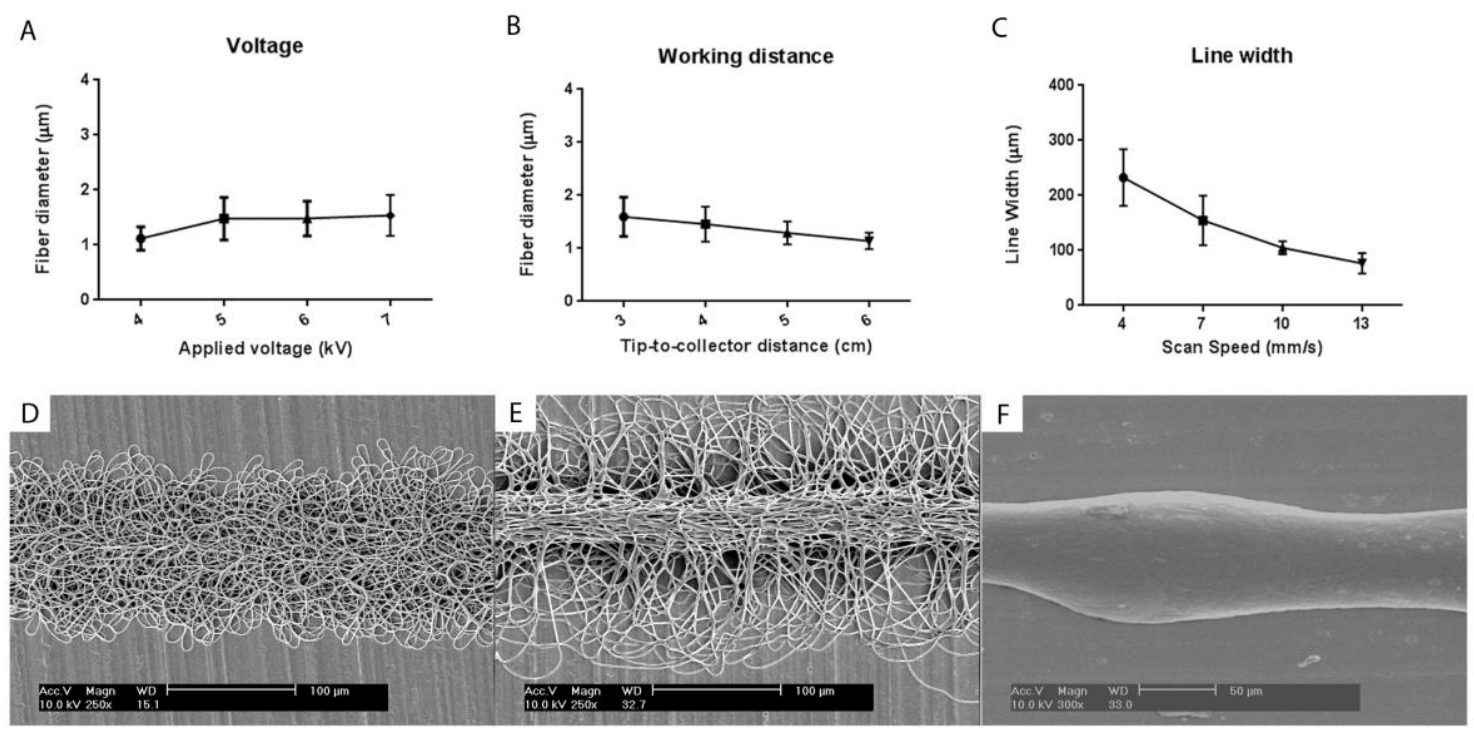

Figure 2. (A-C) Influence of the ESP processing parameters on fiber diameter ( $A$ and $B)$ and line width $(C)$. Applied voltage (A), working distance (B), and collector speed (C) were varied. (D-F) SEM micrographs of different possible configurations of the fiber bundle within the DW range: (D) Bundle with dispersed fibers $(V=5 k V$ and $W d=3 \mathrm{~cm})$; (E) Bundle with a core of aligned fibers $(V=6 k V$ and $W d=3 \mathrm{~cm})$; (F) Single fiber $(\mathrm{V}=4.5 \mathrm{kV}$ and $\mathrm{Wd}=2 \mathrm{~cm})$. Scale bars: $\mathrm{D}$ and $\mathrm{E}(100 \mu \mathrm{m})$ and $\mathrm{F}(50 \mu \mathrm{m})$.

\subsubsection{Optimal ESP parameters}

The range of processing parameters that permitted DW ESP of PEOT/PBT is summarized in Table S3. We found that the optimal range of polymer concentration was from 27 to $29 \%(w / v)$, which yielded fairly viscous solutions. Wd of $\leq 8 \mathrm{~cm}$ were necessary to collect the jet in its stable region, with corresponding optimal voltages of $\leq 8$ $\mathrm{kV}$. Flow rates between 0.08 and $0.3 \mathrm{~mL} / \mathrm{h}$ were necessary and the scan speeds had to be kept above $5 \mathrm{~mm} / \mathrm{s}$. The optimal temperature was kept $\sim 20^{\circ} \mathrm{C}$ and relative humidity was ranged from $40 \pm 2 \%$.

\subsubsection{Pattern fabrication}

Having established the optimal parameters of DW ESP for PEOT/PBT, we proceeded to design scaffolds. The design of a scaffold, such as its architecture and pore network, 
determines its success in a targeted TE application. While the architecture may guide and influence cartilaginous tissue formation[34], porosity is essential not only for nutrient diffusion and waste removal[5, 26], but also allows and impacts cartilage ECM formation[35, 36]. Conventional ESP processes lack on-demand patterning ability and typically produce compact meshes which hinder cell infiltration and ECM formation throughout their volume[18].
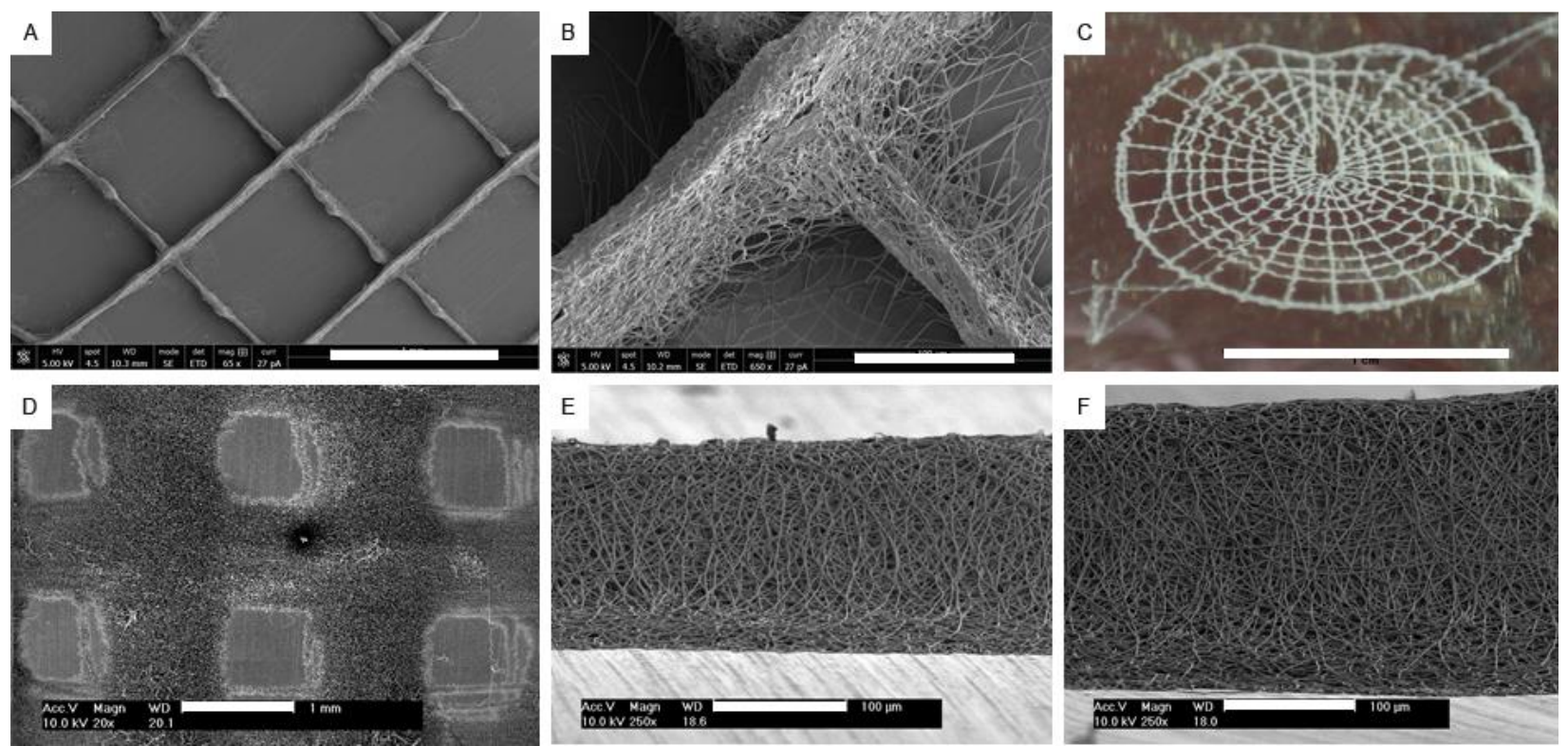

Figure 3. Pattern fabrication with DW ESP. (A and B) SEM micrographs of a $0 / 90^{\circ}$ lay down pattern (A) and detail of a strut intersection (B). (C) Photo of a tympanic membrane design. (D-E) SEM micrographs showing $0 / 90^{\circ}$ lay down pattern with $800 \mu \mathrm{m}$ pore space with 80 layers (110 $\pm 9.0 \mu \mathrm{m}$ thick) (D), 100 layers $(140.5 \pm 8.9 \mu \mathrm{m})(\mathrm{E})$, and 200 layers $(190.8 \pm 20.8 \mu \mathrm{m})(\mathrm{F})$. Scale bars: A and D $(1 \mathrm{~mm})$; $\mathrm{B}(100 \mu \mathrm{m}), \mathrm{C}(1$ $\mathrm{cm})$ and $\mathrm{E}$ and $\mathrm{F}(100 \mu \mathrm{m})$.

DW ESP permits the tailored patterning of 3D ultrathin scaffolds with multiscale and controllable porosity, with a setup that dispenses any complex add-ins. Applying the optimized DW ESP parameters we fabricated regular structures with a $0 / 90^{\circ}$ lay down pattern (Figure 3A-B). The accuracy of this process also allowed the design and fabrication of more complex structures, such one that mimics the anatomy of a tympanic membrane (Figure $3 \mathrm{C}$ ). By tuning the distance between struts and the number of deposited layers, the pore size and scaffold thickness could be controlled. True 3D structures could be built, where $0 / 90^{\circ}$ scaffolds with an approximate fiber space of 800 $\mu \mathrm{m}$ were fabricated with varying thickness based on the number of deposited layers: 110 $\pm 9.0 \mu \mathrm{m}$ with 80 layers (Figure 3D), $140.5 \pm 8.9 \mu \mathrm{m}$ with 100 layers (Figure 3E), and $190.8 \pm 20.8 \mu \mathrm{m}$ with 200 layers(Figure $3 \mathrm{~F}$ ). The fabrication of scaffolds in the centimetre thickness range was hindered by the loss of the macroporous structure. As fibers were 
stacked, both the height and the width of the line pattern increased. This is attributed to a higher concentration of fibers on a single spot and consequently the accumulation of repulsive charges, leading to dispersion during the deposition process[37].

To circumvent this issue, some strategies can be implemented. A stepwise approach of the layer-by-layer deposition could provide enough time for the dissipation of the residual charges, hence reducing the repulsive effect and yielding a more focused deposition. However, if the ESP process is interrupted between the fabrication of layers, multiple jet ejections are required. This is a limiting factor, since minor deviations can occur from the focusing spot, causing a mismatch between the deposited layers. However, if the ESP process is continued, the ejection time will be greatly enhanced and further jet instabilities may arise. Another strategy could be the hybridization of this system with a mechanism for charge compensation used in other applications, such as scanning electron microscopy[38, 39]. Ji et al. reported a noticeable charge decrease on $\mathrm{Al}_{2} \mathrm{O}_{3}$ samples by employing an oxygen atmosphere through a local oxygen pressure device[39]. Finally, another possible solution is to invert the electrode polarity between layer depositions. In such a setup, the residual charges on the deposited fibers would be the opposite of the charges from the upcoming fibers, thus resulting in an attractive effect. Xu et al. followed this strategy and reported an increase in nanofiber deposition accuracy in an NFESP device[37].

\subsubsection{Cell seeding and proliferation on DW ESP scaffolds}

Electrospun meshes generally facilitate cell attachment and distribution due to their ECM resemblance[6]. To determine whether our DW ESP scaffolds supported cell seeding and proliferation, we fabricated scaffolds with fiber spacing of $860 \mu \mathrm{m}$ and an average line width of $150 \mu \mathrm{m}$, then seeded them with hMSCs (Figure S6C). For comparison, we fabricated scaffolds with similar dimensions by 3D printing (Figure S6B). Seeding efficiency was evaluated by DNA quantification 1 day post-seeding (Figure S6A) and revealed that DW ESP scaffolds promoted 7 times more cell retention than those fabricated by AM techniques (17.66\% v. 2.43\%). To assess cell distribution and proliferation, the scaffolds were stained with methylene blue after 1 and 4 days of culture (Figure S7). At day 1, cells seeded on the 3D-printed scaffolds were mostly retained at the fiber intersections (Figure S7A), while those seeded on the DW ESP scaffolds were more abundant and well distributed (Figure S7C). At day 4, both scaffolds supported cell proliferation, but a larger population was present on the DW ESP structure (Figure S7B 
and D). These data argue that the DW ESP scaffolds promoted better cell seeding and retention compared to the 3D-printed scaffolds.

\subsubsection{Fabrication of an articular cartilage mimetic scaffold}

Having established that DW ESP could be used to fabricate scaffolds that support cell seeding and proliferation, we then tested whether this novel technique could be used to create a scaffold that mimics articular cartilage with its distinct multi-zone morphology (Figure 4A); such a scaffold may offer a superior template for in vitro cartilage formation and ECM organization[40]. We fabricated a scaffold that recapitulates three of the four main zones of cartilage (Figure 4B-E). The superficial zone, denoted by tangentially oriented collagen fibrils, was represented by two parallel electrospun layers with an average distance of $238 \pm 79.3 \mu \mathrm{m}$. To reproduce the oblique collagen structure of the middle zone, we created crossed diagonal layers. Finally, the deep zone was represented by parallel layers, radially oriented to the top structure, with an average distance of $523 \pm$ $156.2 \mu \mathrm{m}$. The mimetic scaffolds had pore dimensions ranged from $238.0 \pm 79.3$ to 523.6 $\pm 156.2 \mu \mathrm{m}$ of which the values were within the ideal dimensions (250 to $500 \mu \mathrm{m}$ ) reported for chondrocyte proliferation and ECM secretion on gelatin scaffolds[41]. Detailed views of the fiber bundle and individual ultrathin fibers with an average diameter of $1.39 \pm 0.6 \mu \mathrm{m}$ (Figure S8) are shown in Figure 4D and 4E, respectively.

Next, we further characterized the mimetic scaffold's physical and mechanical properties (Table S4). Overall, the measured porosity was $91.2 \pm 1.0 \%$ (Table S4), which is fairly large compared to other structures for the same application[34]. The mechanical properties of the scaffolds were also characterized using a nanoidenter. The measured Young's modulus in compression was $2.65 \pm 0.65 \mathrm{MPa}$, which proved to be higher than human native cartilage as measured by in situ biphasic creep indentation $(0.45 \mathrm{MPa}$ to $0.80 \mathrm{MPa}$ ) or bovine cartilage measured by nanoidentation[42, 43].

In order to engineer a larger tissue construct, four individual scaffolds (each approximately $208.5 \pm 19.4 \mu \mathrm{m}$ thick) were stacked and bonded to yield an assembled structure of $1.39 \pm 0.6 \mathrm{~mm}$ in height. The introduction of sacrificial layers of PEO between the spun mats promoted lateral pore formation, and the bonding process did not affect the inner core of the scaffold (data not shown). 
A
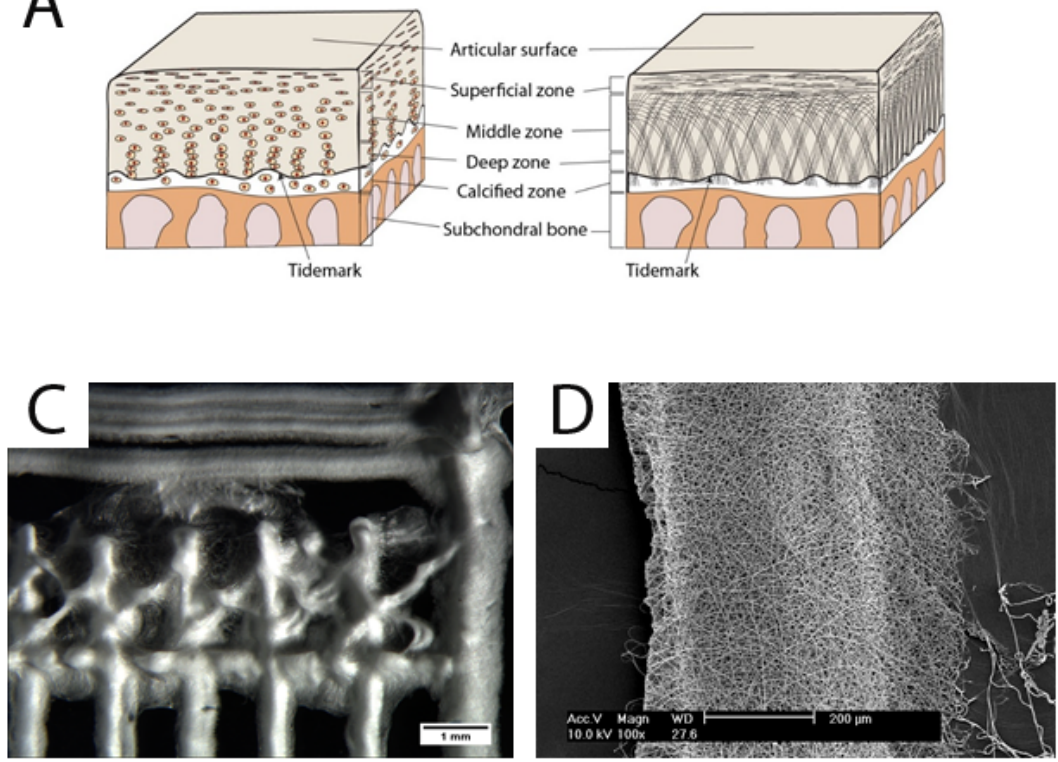
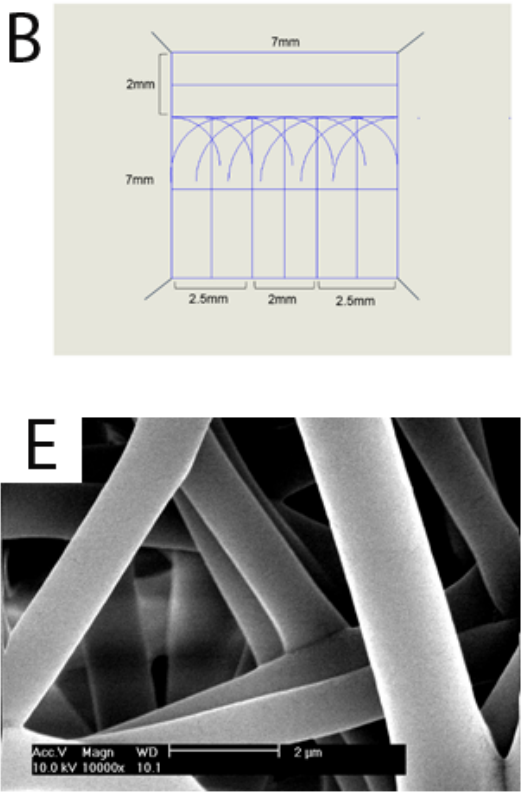

Figure 4. Design of an articular cartilage mimetic scaffold. (A) Illustration of the zonal configuration in native cartilage[44]; (B) CAD design of the devised scaffold; (C) stereomicroscope image of the DW ESP structure, depicting the different zones; ( $D$ and $E$ ) SEM micrographs of the fiber bundle corresponding to the bottom columns of the scaffold (D) and detailed view of individual fibers at $100 \times$ magnification $(E)$. Scale bars: C $(1 \mathrm{~mm}), \mathrm{D}(200 \mu \mathrm{m})$, and $\mathrm{E}(2 \mu \mathrm{m})$.

\subsubsection{Cell culture and chondrogenic differentiation}

To investigate the potential of designed scaffolds for articular cartilage regeneration, we cultured hMSCs on stacked DW ESP scaffolds (unless otherwise stated) in chondrogenic and basic medium (negative control medium). As a control, cell-seeded random fiber (RF) scaffolds were also prepared and analyzed. The cell proliferation on the DW and RF seeded scaffolds was evaluated at three time points during the first week of culture, using a Presto Blue assay (Figure S9). Proliferation was registered in all the conditions through an increase in fluorescent signal. In both media, DW scaffolds showed 4-fold higher intensity compared to RF scaffolds, until day 7 when cell proliferation increased steeply on RF sheets. This observation can be explained by the higher number of fibers in the vicinity of cells, due to the lack of large pores on the RF structure, which leads to easier cell spreading, and thus a faster proliferation rate. For chondrogenic differentiation, cells on DW scaffolds showed a steady increase in fluorescence over the 7 days. In contrast, the signal from cells incubated on RF scaffolds in differentiation medium showed little change over the same period. These data indicate that cells proliferated on both RF and DW scaffolds, 
To determine the topographical influence of DW mimetic scaffolds on tissue development and matrix secretion, scanning electron microscopy (SEM) observations were performed after 21 days of culture in chondrogenic medium. Whereas the RF and DW scaffolds both demonstrated full coverage of cells, only the DW scaffolds showed cells and matrix throughout its volume (Figure S10).
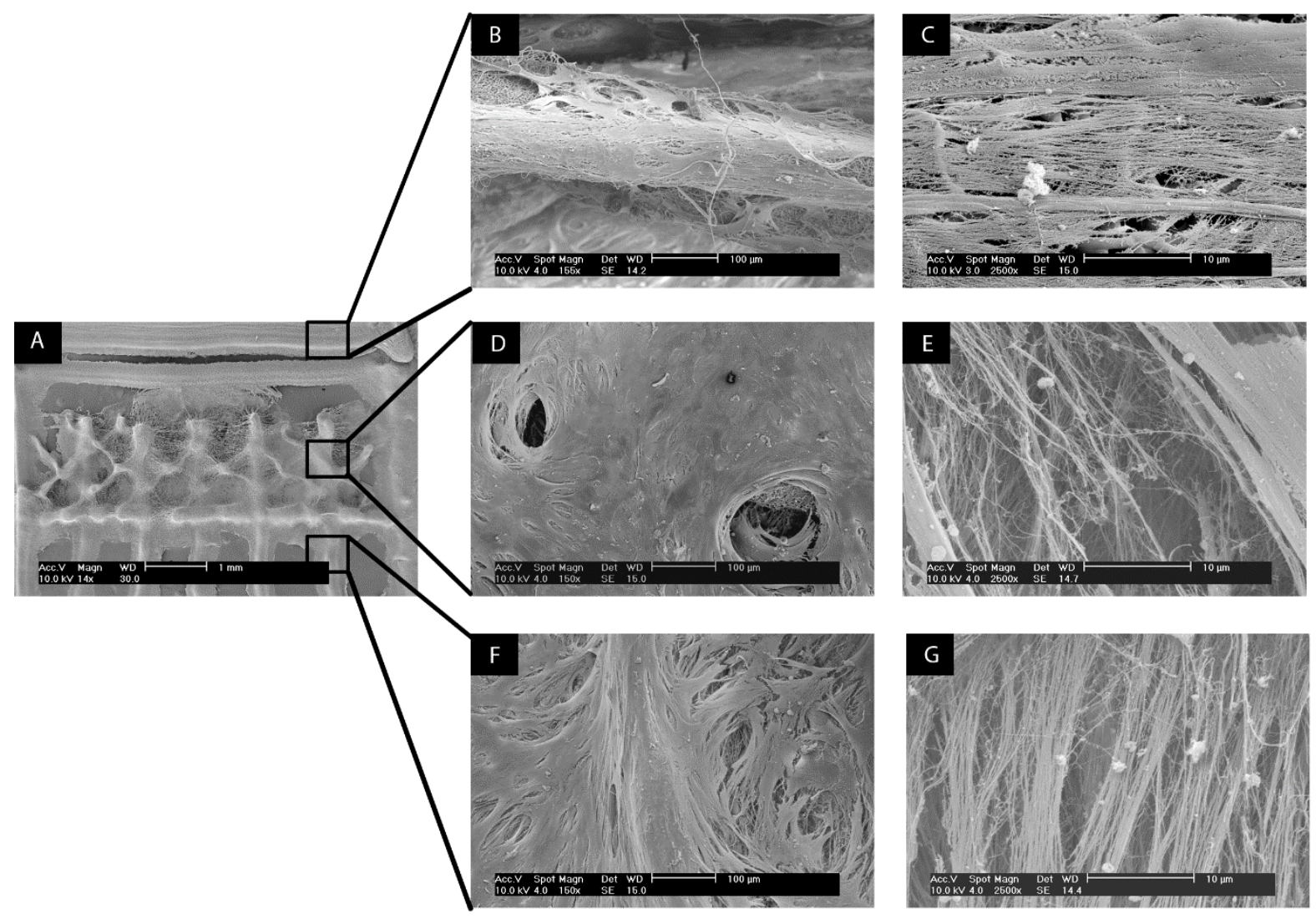

Figure 5. SEM images showing the topographical influence of the scaffold's architecture on tissue development (B, D, F) and matrix orientation (C, E, G) after 21 days of culture in chondrogenic medium. (A) Empty scaffold fabricated by DW ESP. Low-magnification images $(B, D, F)$ and high-magnification images $(C, E, G)$ of the superficial zone $(B-C)$, middle zone $(D-E)$, and deep zone $(F-G)$. Scale bars: $B, D$ and $F$ $(100 \mu \mathrm{m}), \mathrm{C}, \mathrm{E}$ and $\mathrm{G}(10 \mu \mathrm{m})$.

When cultured on the RF mesh, hMSCs dispersed randomly and as a consequence their secreted matrix was isotropically distributed (data not shown). In comparison, the matrix organization was influenced by the zonal topography of the DW scaffolds (Figure 5). The horizontal layers forming the superficial zone (Figure $5 \mathrm{C}$ ) and the vertical layers of the deep zone (Figure $5 \mathrm{G}$ ) promoted tissue development along their respective axes, which resulted in the anisotropic formation of fibrils. Coherence analysis of the SEM micrographs showed a slightly higher coefficient of directionality for the deep zone $(0.49 \pm$ $0.08)$ compared to the superficial zone $(0.41 \pm 0.09)$ (Figure S11). 
The oblique layers that represent the middle zone, induced tissue formation in a more random manner with secreted fibers displaying a crossed pattern (Figure 5E). The coefficient of directionality for this middle zone was $0.33 \pm 0.11$ (Figure S11). The presence of holes (Figure 5D) suggested the formation of tissue in different and opposite directions. In summary, the DW scaffold imparted a tissue and matrix organization in a zone-dependent way, in contrast to the isotropy seen in the RF sheets.

The morphology and feature size of the fibrils observed in the SEM micrographs suggested the presence of collagen. Among collagen types, type II collagen is the basis for articular cartilage. To investigate the formation of collage type II, the surface of the scaffolds was immunostained against collagen type II after 21 days of culture in chondrogenic medium, but no signal was detected. hMSC pellets after 21 days in chondrogenic medium showed collagen type II staining (Figure $\mathrm{S} 12 \mathrm{C}-\mathrm{F}$ ), suggesting that pellet conditions (such as greater cell-cell contact, maintenance of cell rounded shape, softer substrate, and reduced oxygen tension), resulted in improved differentiation of hMSCs and consequent production of collagen type II45-47]. We are investigating whether modifications to the cell culture conditions that provide an environment more similar to the pellet conditions such as using a hydrogel for scaffold coating or a cell carrier like hyaluronic acid (Figure S13) will improve chondrogenic differentiation and collagen I and II production. When cultured on flat substrates, differentiated chondrocytes might dedifferentiate into fibroblast-like cells and produce collagen type I instead[48]. Therefore, we further immunostained the scaffolds for collagen type I (Figure 6). We observed distinct collagen type I staining that resembled the matrix patterning. The RF scaffold revealed random, non-preferential direction collagen type I staining (Figure 6A). For the DW scaffold, collagen type I anisotropy was observed for the top (Figure 6B) and bottom layers (Figure 6D), and an arc-like configuration seen for the middle layer (Figure $6 \mathrm{C})$. These results indicate that the DW scaffold enabled collagen matrix organization in a zone-dependent way in contrast to the isotropy seen in the RF mesh. 

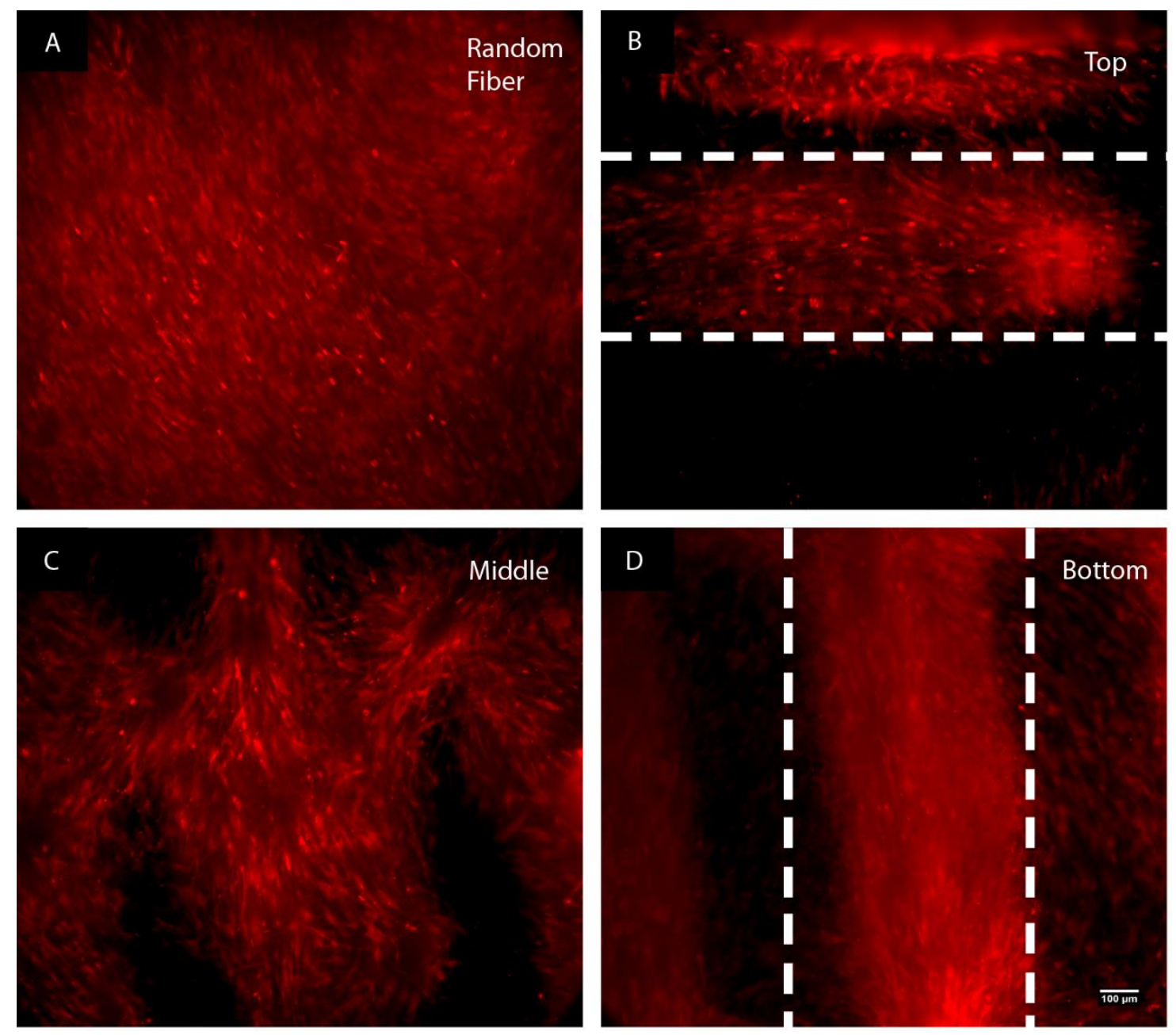

Figure 6. Immunostaining of collagen type I on hMSCs seeded scaffolds after 21 days of culture in chondrogenic medium. (A) RF scaffold; (B-D) Sections of the DW scaffold, as labelled. Scale bar in D (100 $\mu \mathrm{m})$ applies to all panels. The dashed lines in panels $B$ and $D$ delineate the collagen fiber orientation.

To determine whether the observed patterns in SEM were cartilage-specific matrix, we visualized sulphated glycosaminoglycan (GAG) by staining the scaffolds with Alcian Blue after 21 days of culture. Cultured cell pellets were also stained as a positive control (Figure S12A-B). As shown in Figure S14, both RF and DW scaffolds present higher amount of GAG staining in chondrogenic medium compared to basic medium(control medium). In chondrogenic medium, GAG staining was abundant and distributed all over the RF scaffold (Figure 7A). For the DW scaffolds, we observed GAG staining in a zonal distribution and distinct cell morphology (Figure 7B-D). For the top (Figure 7B) and bottom (Figure 7D) layers of the scaffolds, we observed anisotropic GAG staining and cell elongation along the same direction. This was particularly evident in the top layer, with the presence of some flattened cells on the most superficial zones, which resembled the native state. GAG staining was more randomly dispersed in multiple directions for the 
middle section (Figure 7C), and the cells were more rounded compared with the other scaffold zones.
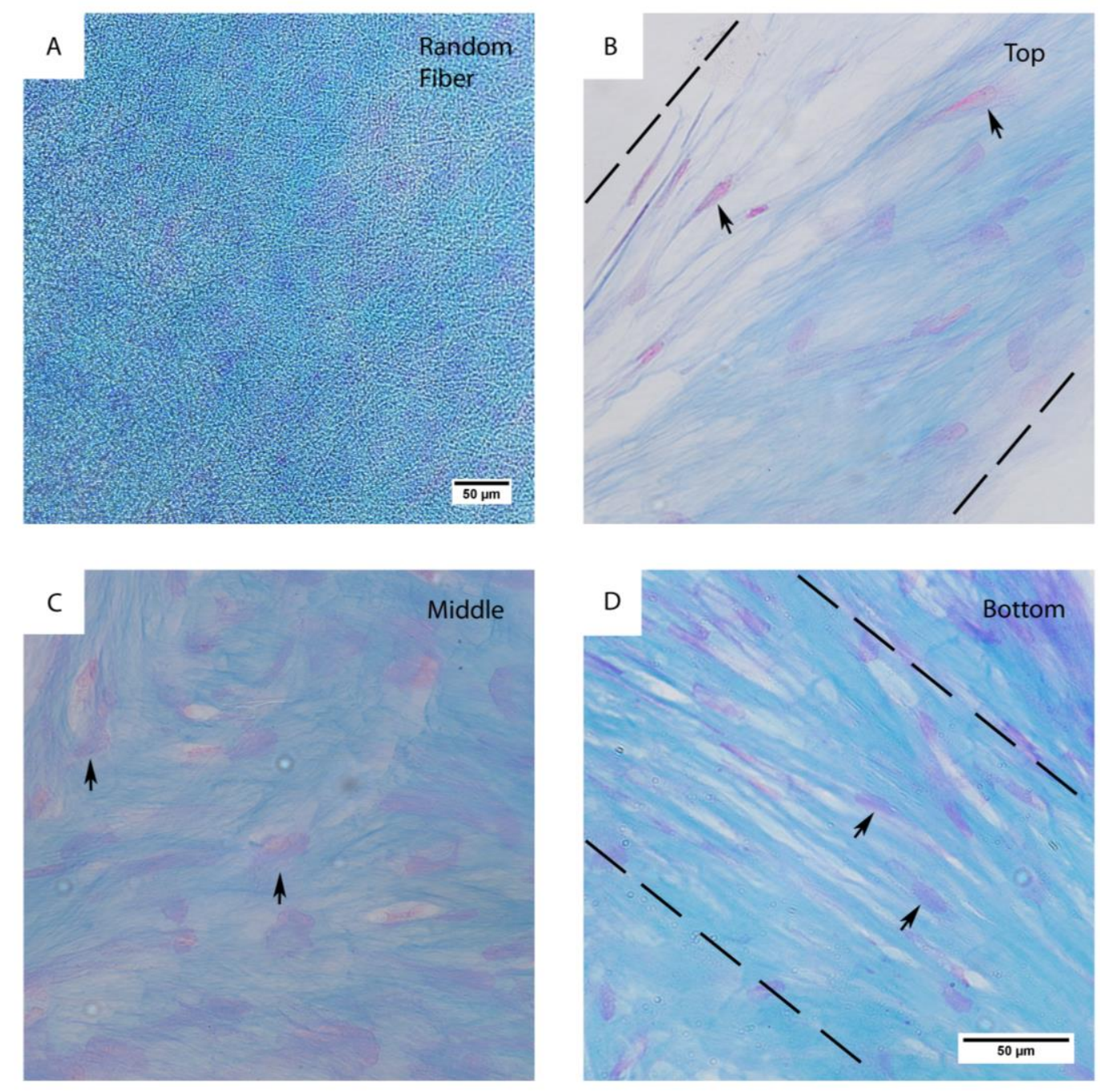

Figure 7. Staining with Alcian Blue for sulphated GAGs and Nuclear Fast Red for cell nuclei in histological sections of hMSCs after 21 days of culture in chondrogenic medium on RF (A) and DW (B-D) scaffolds. (A) $10 \times$ view of the RF scaffold; (B-D) $20 \times$ view of the top (B), middle (C), and bottom (D) sections of DW ESP scaffolds. The dashed lines represent the direction on the layer (parallel to its axis) and the arrows point to cells. Scale bar in $D$ also applies to panels B and C.

Quantification of GAG production showed similar levels for both scaffolds (Figure 8A). However, cell adhesion and proliferation were significantly greater in DW scaffolds compared to RF sheets (Figure 8B). As a consequence, the RF sheets exhibited significantly higher GAG/DNA ratios (Figure $8 \mathrm{C}$ ), or more matrix production normalized to cell content, compared to DW scaffolds. Although seeded at the same density (per volume), RF sheets may achieve cell confluency at earlier time compared to DW scaffolds due to their smaller available growth area. Therefore, cells grown on RF 
scaffolds were subjected to higher cell-cell interaction throughout the culture period. Reports in literature show that higher cell densities, as a consequence of higher cell-cell interaction, enhance matrix production in cultures with hMSCs[49] and articular chondrocytes[50], which could explain our observed differences. Quantification of GAG and DNA levels after cells achieve full confluency on the scaffolds may help clarify this difference.

Finally, the cell-seeded scaffolds were examined for the presence of several chondrogenic markers, including Collagen type II alpha 1 (Col2a), Sox9 and aggrecan (ACAN). Col2a and ACAN are major proteins found in cartilage matrix[2, 51]. Sox9 is a transcription factor that plays a key role in chondrogenes[52, 53]. The cell-seeded scaffolds cultured in basic medium were used as control. Levels of mRNA for all three markers were higher for DW scaffolds in chondrogenic medium compared to basic medium (Figure 8D-F). In chondrogenic medium, expression of Sox9 and ACAN was significantly higher in DW scaffolds compared to RF scaffolds after both 14 and 21 days of culture (Figure 8E-F). However, the higher expression of Sox9 and ACAN did not always result in a higher expression of Col2a. Col2a expression was higher for the RF scaffolds compared to the DW scaffolds at day 21 in chondrogenic medium (Figure 8D). This uncoupling of Col2a and Sox9 expression was also reported by Aigner et al. who demonstrated that Sox9 was not the central regulatory mediator of Col2a in human articular chondrocytes[54]. They demonstrated that high gene expression of Sox9 did not lead to an increase of Col2a expression in normal articular chondrocytes and the amount of Col2a expression was higher in spite of reducing Sox9 expression in osteoarthritic chondrocytes. The higher expression of Sox9 in DW scaffolds could be responsible for the higher expression of ACAN in DW scaffolds. Previous studies demonstrated that Sox9 supported the aggrecan promoter activity in clonal chondrocytic cells[53]. These data argue that DW scaffolds supported chondrogenic differentiation of hMSCs. 

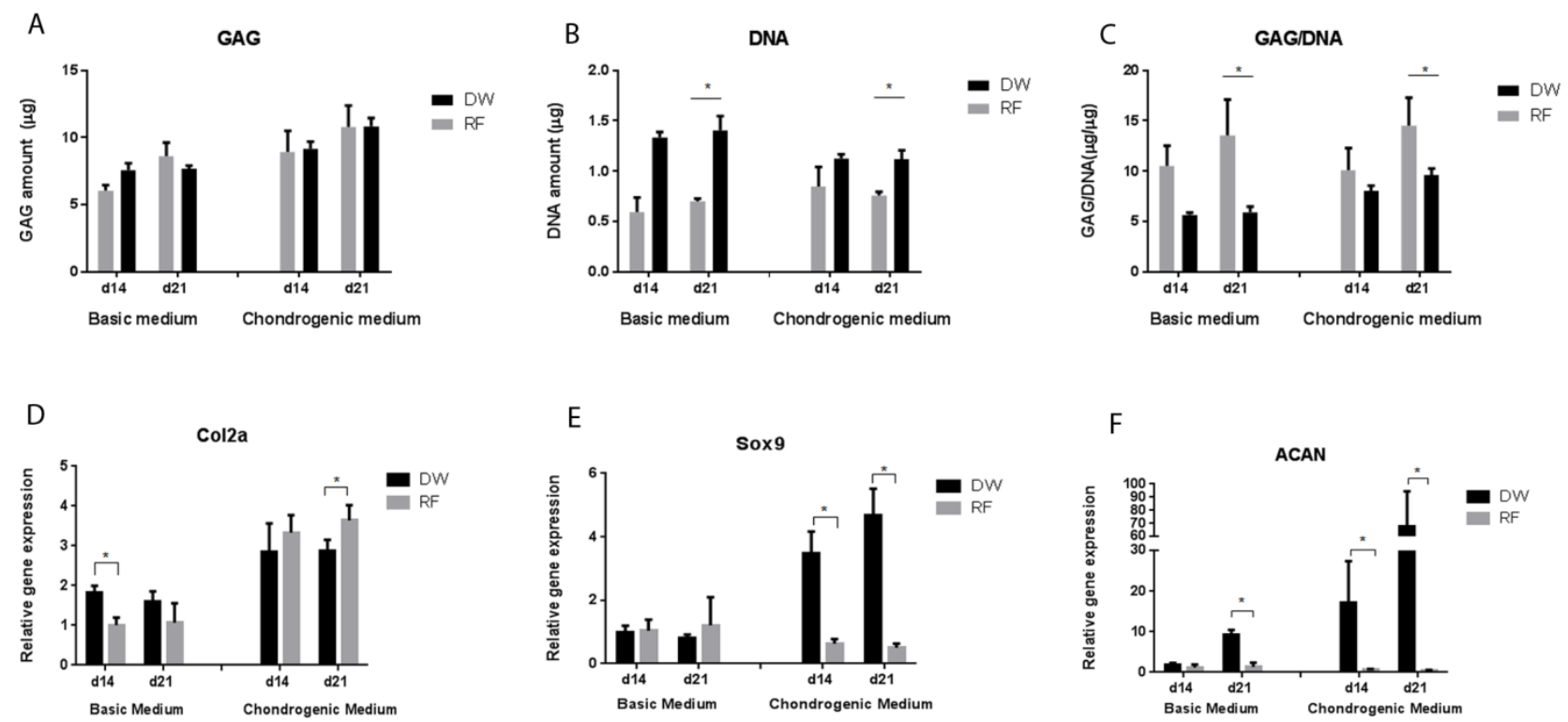

Figure 8. Cartilaginous matrix production $(A-C)$ and gene expression profiles (D-F) of hMSCs cultured for 14 and 21 days in basic and chondrogenic medium. (A) Total GAG amount; (B) total DNA amount, and (C) GAG normalized to the DNA content. mRNA levels of Col2A (D), Sox9 (E), and ACAN (F) are represented as fold differences relative to the expression obtained in RF samples cultured in basic medium at day 14 . ${ }^{*} p<0.05$.

\subsection{Conclusion}

We have shown here the development of a technique for direct writing with electrospinning, with capability to control the fiber bundle morphology and fabricate regular 3D ultrathin structures. Applying this technique, we produced a scaffold that mimics the zonal organization of articular cartilage and compared its performance for cartilage tissue formation to a regular electrospun sheet. In contrast to nonspecific deposition by the typical electrospun mesh, DW scaffolds were able to direct tissue organization and fibril matrix orientation. Expressions of chondrogenic markers, including Sox9 and ACAN, were also significantly enhanced in this structure. Our work provides a new technique for the patterning of nano/microfibrous structures with potentially wide application for the engineering of many tissues, and particularly those presenting regional differences. Further optimization might enable the fabrication of articular cartilage suitable for therapeutic applications. 


\subsection{Materials and Methods}

\subsubsection{Polymer solution preparation}

A PEOT/PBT block copolymer (PolyVation B.V.; the weight ratio of PEOT/PBT=55/45, the molecular weight $(\mathrm{g} / \mathrm{mol})$ of the starting PEG segments used in the polymerization process is 300$)$ was dissolved in a mixture of chloroform $\left(\mathrm{CHCl}_{3}\right.$, Sigma-Aldrich) and 1,1,1,3,3,3-hexafluoro-2-propanol (HFIP, Biosolve-Chemicals) to prepare the polymer solutions. Solutions of 26 to $30 \%$ of polymer $(\mathrm{w} / \mathrm{v})$ in $70: 30$ to $80: 20 \mathrm{CHCl}_{3} / \mathrm{HFIP}(\mathrm{v} / \mathrm{v})$ were formulated.

\subsubsection{Electrospinning setup}

The ESP system configuration consisted of a two parallel plate setup, composed by a top unit $(21.5 \times 30 \mathrm{~cm})$ of a $2 \mathrm{~cm}$ thick copper plate encased in Teflon and a $0.5 \mathrm{~cm}$ thick copper collector $(10 \times 10 \mathrm{~cm})$ mounted on an $X Y$ translational stage. The stage is a modified 3D printer (k8200, Velleman), specifically customized for ESP experiments and controllable using Repetier-Host software (v0.84, Repetier).

\subsubsection{Electrospinning tests and jet imaging}

Initial electrospinning experiments to assess the drawing region were carried with polymer solutions of 26 to $30 \%$ PEOT/PBT in $70: 30$ to $80: 20 \mathrm{CHCl}_{3} / \mathrm{HFIP}(\mathrm{v} / \mathrm{v})$. The applied voltage was varied from 2.0 to $16 \mathrm{kV}$, the working distance from 2 to $16 \mathrm{~cm}$, the flow-rate from $0.05 \mathrm{~mL} / \mathrm{h}$ to $2 \mathrm{~mL} / \mathrm{h}$, the temperature from $18.5{ }^{\circ} \mathrm{C}$ to $30{ }^{\circ} \mathrm{C}$ and the relative humidity from $25 \%$ to $55 \%$. The scan-speed was varied from 1 to $13 \mathrm{~mm} / \mathrm{s}$ and spinneret diameter were fixed to $0.5 \mathrm{~mm}$. After jet initiation, the collector was set to a linear motion to collect the fibers in a single direction. A gross visual examination of the outcome and scanning electron microscope (SEM) analysis were done to analyse the contribution of each parameter to the jet stability and to define the parameters range for drawing capability.

ESP tests within the drawing region were carried with a 28\% PEOT/PBT in 75:25 $\mathrm{CHCl}_{3} / \mathrm{HFIP}(\mathrm{v} / \mathrm{v})$. The applied voltage varied from 4 to $8 \mathrm{kV}$, the working distance from 2 to $7 \mathrm{~cm}$ and the scan-speed from 1 to $13 \mathrm{~mm} / \mathrm{s}$. The flow-rate was set to $0.18 \mathrm{ml} / \mathrm{h}$ through a spinneret with $0.8 \mathrm{~mm}$ diameter. A temperature of $20^{\circ} \mathrm{C}$ and a relative humidity of $30 \%$ were used as ambient parameters. SEM micrographs were then taken to investigate the fiber bundle morphology within the drawing region 
Images of the jet flight were captured using a digital camera (Panasonic Lumix DMC G3) and the resulting photographs were further treated with ImageJ.

\subsubsection{Direct writing and scaffold fabrication}

Direct writing experiments and scaffold fabrication were carried with a polymer solution of 28\% PEOT/PBT in 75:25 CHCl3/HFIP (v/v) with the experimental parameters listed on Table S5. For the fabrication of the assembled scaffolds, the electrospun mats were stacked via a manual process illustrated in Figure S15. Briefly, the scaffolds were detached from the aluminium foil by placing them in deionized (DI) water for 1 to $2 \mathrm{~min}$ and gently tapping the structure with a tweezers to promote detachment. After drying with filter paper, the scaffolds were placed on top of a polydimethylsiloxane (PDMS) block covered with a Teflon sheet, and held in place with thin metal wires. Overall, four scaffolds were stacked together to achieve the final constructs. To promote lateral pore formation, a thin poly (ethylene oxide) (PEO, Mw=900,000 g/mol, Sigma-Aldrich) sheet (300 $\mu \mathrm{m}$ thick) fabricated through solvent casting of $3 \%$ PEO in $80: 20 \mathrm{H} 2 \mathrm{O} / \mathrm{ethanol}(\mathrm{v} / \mathrm{v})$, was placed between each scaffold. The stacked layers were then bound together by joining the vertexes of each scaffold and melting them at $180^{\circ} \mathrm{C}$ (using a micro soldering iron). The assembled constructs were then placed overnight in a container filled with $\mathrm{DI}$ water under agitation to dissolve the sacrificial PEO layers. Following this process, the scaffolds were retrieved and let to dry.

The fabrication of random fiber (RF) sheets was carried using the same polymer solution and setup, with the process parameters listed on Table S5, which were selected to fabricate a mesh with similar fiber dimensions as the DW scaffold. The collector was set to a raster scan path with a step size on the $Y$ axis of $1 \mathrm{~mm}$ to ensure thickness uniformity on the deposited mesh. Scaffolds with a diameter of $12 \mathrm{~mm}$ were then punched out for further cell culture study.

\subsubsection{Scaffold characterization}

The scaffold geometry and architecture was characterized through measurements on SEM micrographs. The height of the final assembled construct was measured using a digital caliper. The porosity of the electrospun scaffold was calculated using the theoretical approach described in equation 1 : 
$\operatorname{Porosity}(\%)=\left(1-\frac{m_{\text {scaffold }}}{\rho_{\text {material }} V_{\text {scaffold }}}\right) \times 100 \%$

Equation 1

Where $\mathrm{m}_{\text {scaffold }}$ is the measured weight of the scaffold, Pmaterial is the density of the material $\left(1.2 \mathrm{~g} / \mathrm{mL}\right.$ )[55] and $V_{\text {scaffold }}$ is the apparent volume of the scaffold (assuming a solid block of equal dimensions). To determine the Young's modulus under compression of the electrospun scaffolds, a mechanical test was performed using a nanoidentation device (Piuma, Optics 11). The compression test on the scaffold was carried with a tip of radius (r) of $22.5 \mu \mathrm{m}$ and spring constant $(\mathrm{k})$ of $78.3 \mathrm{~N} / \mathrm{m}$. 40 indentations were performed with a step size of $50 \mu \mathrm{m}$ on the $X$ axis and $5 \mu \mathrm{m}$ on the $Y$ axis.

\subsubsection{Cell culture}

Pre-selected hMSCs (male age 22) were retrieved from the Institute of Regenerative Medicine at Texas A\&M University [56, 57]. Briefly, a bone marrow aspirate was drawn from the patient after informed written consent, and mononuclear cells were separated using density centrifugation. hMSCs at passage 2 were expanded in basic medium (BM) composed of a-MEM (Thermo Fisher Scientific) supplemented with $10 \%$ fetal bovine serum (Lonza), $2 \mathrm{mM}$ L-glutamin (Thermo Fisher Scientific), $0.2 \mathrm{mM}$ ascorbic acid (Sigma-Aldrich) and 100 units $/ \mathrm{mL}$ penicillin plus $100 \mathrm{mg} / \mathrm{mL}$ streptomycin (Thermo Fisher Scientific) until reaching $80-85 \%$ confluence. The medium was refreshed every 2 days.

\subsubsection{Pellet culture}

To check the donor potential for chondrogenesis, a pellet culture study was performed over 21 days as a positive control. 2x105 cells suspended in $200 \mu \mathrm{L}$ of chondrogenic medium composed of DMEM (Thermo Fisher Scientific) supplemented with $1 \%$ ITS premix (Micronic BV), $50 \mu \mathrm{g} / \mathrm{mL}$ ascorbic acid (Sigma-Aldrich), $40 \mu \mathrm{g} / \mathrm{mL}$ of proline (Sigma-Aldrich), $100 \mu \mathrm{g} / \mathrm{mL}$ of sodium pyruvate (Life Technologies), $100 \mathrm{units} / \mathrm{mL}$ penicillin plus $100 \mathrm{mg} / \mathrm{mL}$ streptomycin (Thermo Fisher Scientific), $0.01 \mu \mathrm{g} / \mathrm{mL}$ of TGF- $\beta 3$ (R\&D systems) and $100 \mathrm{nM}$ dexamethasone (Sigma-Aldrich) were seeded in round bottomed non-treated tissue culture 96-well plate. For pellet formation, the cells were centrifuged at $2000 \mathrm{rpm}$ for $3 \mathrm{~min}$ at $4{ }^{\circ} \mathrm{C}$. As a control the cells were seeded in BM using the same protocol. The medium was refreshed every 2 days, taking out $100 \mu \mathrm{L}$ and adding $100 \mu \mathrm{L}$. The pellets were fixed in $4 \%$ paraformaldehyde for $2 \mathrm{~h}$ at $4{ }^{\circ} \mathrm{C}$, and then washed three times with phosphate buffered saline solution (PBS) and maintained wet. 6.4.8. Seeding efficiency and proliferation on 3D-printed $v$. DW ESP scaffolds 
3D printed scaffolds made of PEOT/PBT polymer were fabricated via additive manufacturing (Bioscaffolder, SysENG, Germany) [35]. hMSCs were seeded on DW ESP and 3D printed scaffolds at a density of $5 \times 10^{4}$ cells $/ \mathrm{cm} 2$, and cultured in BM during 4 days, with medium changes every other day. Cell attachment was evaluated by DNA quantification using CyQUANT® Cell Proliferation Assay Kit (Thermo Fisher Scientific). Briefly, the scaffolds were washed three times with PBS, transferred to a $1.5 \mathrm{~mL}$ Eppendorf tubes and placed at $-80^{\circ} \mathrm{C}$ overnight. After this, three freeze/thaw cycles (from $-80{ }^{\circ} \mathrm{C}$ to room temperature) were applied and $250 \mu \mathrm{L}$ of proteinase $\mathrm{K}$ digestion buffer $(0.001 \%(w / v)$ proteinase $\mathrm{K}, 10 \%$ of $1.85 \mathrm{mg} / \mathrm{mL}$ iodoacetamide (Sigma-Aldrich) solution and $10 \%$ of $100 \mu \mathrm{g} / \mathrm{mL}$ of pepstatin A (Sigma-Aldrich) solution in Tris/EDTA buffer) was added to every tube, followed by incubation at $56^{\circ} \mathrm{C}$ for $16 \mathrm{~h}$ under agitation. The lysate was collected and used to measure the DNA amount in the scaffolds using the kit protocol. Cell distribution and proliferation were assessed with methylene blue staining. Briefly, samples were fixed in $10 \%$ formalin solution for $30 \mathrm{~min}$, followed by a rinse with demi water for $1 \mathrm{~min}$. After that, samples were put in $1 \%$ methylene blue solution for $60 \mathrm{~s}$, and washed with demi water again for three times. Images were acquired were a stereomicroscope (SMZ25, Nikon).

\subsubsection{Cell seeding and culture on RF sheets and DW ESP scaffolds}

Prior to cell seeding, samples were placed in a non-treated tissue culture 24-well plate (O-rings were used to prevent scaffolds floating), sterilized in $70 \%$ ethanol for $1 \mathrm{hr}$ and washed three times with PBS. After removing the PBS, the scaffolds were pre-wetted in basic medium overnight for next step cell seeding. Cells were seeded on each scaffold at a similar density (12000 cells/mg of material) unless otherwise stated. After cell attachment, a final volume of $1 \mathrm{~mL} \mathrm{CM}$ was applied for each well. Cell culture on scaffolds in BM was used for negative control. Cell culture on scaffolds was kept for 21 days, and the media refreshed every 2-3 days.

\subsubsection{Scanning electron microscopy (SEM) Analysis}

The cell-seeded scaffolds $(n=4)$ were washed three times with PBS, fixed during $2 \mathrm{~h}$ in $10 \%$ neutral buffered formalin, washed again three times with PBS, dehydrated in a series of ethanol dilutions ( $1 \times 50,60,70,80,90$ and $96 \%$ and $2 \times 100 \%)$ for 30 min and then critical point dried with liquid carbon dioxide (Balzers CPD 030 and Leica EM CPD 300). Following this, samples were gold sputtered (Cressington Sputter Coater 108 auto) 
for 40s at $30 \mathrm{~mA}$ and then imaged using a SEM (Philips XL-30 ESEM, Philips; and Versa $3 \mathrm{D}, \mathrm{FEl}$ ) at a $\mathrm{V}=10 \mathrm{kV}$. For analysis of the fiber diameter and morphology, and characterization of the electrospun scaffolds, the samples were gold sputtered and imaged using the same parameters.

\subsubsection{Metabolic Activity}

The cell metabolic activity was measured at day 1, day 4 and day 7 using the PrestoBlue® Cell Viability Reagent (Life Technologies). Briefly, a 10\% (v/v) presto blue solution in culture medium was added to each sample $(n=4)$ and incubated at $37{ }^{\circ} \mathrm{C}, 5 \%$ $\mathrm{CO}_{2}$ during $2 \mathrm{~h}$, protected from light. After that time, $150 \mu \mathrm{L}$ aliquots were transferred to a black 96-well plate and the fluorescence measured in a Perkin Elmer Victor3 plate reader (Perkin Elmer) using the following parameters for Ex/Em: 560/590 nm. Blanks of medium only were made to correct for background fluorescence.

\subsubsection{Real-time quantitative polymerase chain reaction ( $q P C R$ )}

Gene expression of the chondrogenic markers including Col2a, Sox9, and ACAN was evaluated after 14 and 21 days of culture. Total RNA was isolated from the samples using a TRIzol based extraction method followed by the Isolate II RNA Mini Kit (Bioline). Briefly, $1 \mathrm{~mL}$ of TRIzol reagent (Thermo Fisher Scientific) was added to each sample, followed by centrifugation at 12000 rcf for $2 \mathrm{~min}$ at $4{ }^{\circ} \mathrm{C}$, addition of $0.2 \mathrm{~mL}$ of a CHCl3: isoamyl alcohol mixture and again centrifugation at $12000 \mathrm{rcf}$ for $15 \mathrm{~min}$ at $4^{\circ} \mathrm{C}$. The aqueous phase was then collected and the rest of the procedure was conducted using the Isolate II RNA Mini kit protocol. RNA quantification and purity assessment were done in a NanoDrop 2000 (Thermo Fisher Scientific). cDNA was synthesized using the SensiFAST ${ }^{\mathrm{TM}}$ CDNA Synthesis Kit (Bioline) according to the manufacturer's protocol. qPCR was performed in the CFX Connect ${ }^{\mathrm{TM}}$ Real-Time PCR Detection System (Bio-Rad, UK) using the SensiMix ${ }^{\mathrm{TM}}$ SYBR® \& Fluorescein Kit (Bioline) and the primers listed in Table S6. $25 \mu \mathrm{L}$ of sample volume composed of $3 \mu \mathrm{L}$ of cDNA, $12.5 \mu \mathrm{L}$ of kit reagent, 7.5 $\mu \mathrm{L}$ of distilled water and $1 \mu \mathrm{L}$ of forward and reverse primer were used in the amplification process. For this, cDNA was first denatured for $10 \mathrm{~min}$ at $95^{\circ} \mathrm{C}$, followed by 45 cycles, consisting of $15 \mathrm{~s}$ at $95^{\circ} \mathrm{C}, 15 \mathrm{~s}$ at $60{ }^{\circ} \mathrm{C}, 15 \mathrm{~s}$ at $72{ }^{\circ} \mathrm{C}$. Ct values were determined and normalized to the housekeeping gene B2M and $\Delta \mathrm{Ct}$ ((average of $\left.\left.\mathrm{Ct}_{\text {control }}\right)-\left(\mathrm{Ct}_{\text {value }}\right)\right)$. The relative mRNA of the markers of interest is represented as fold changes relative to the 
expression obtained in the random fiber samples cultured in basic medium and calculated by $2^{-\Delta \Delta C t}$.

\subsubsection{Immunohistochemistry}

Samples $(n=3)$ were retrieved after 21 days of culture, washed three times with PBS and fixed for $30 \mathrm{~min}$ in $4 \%$ paraformaldehyde, at $4{ }^{\circ} \mathrm{C}$. Samples were permeabilized in $0.5 \%$ Triton X-100 for $5 \mathrm{~min}$ at room temperature, followed by incubation with blocking buffer (0.05\% Tween 20, 5\% goat serum, and 1\% BSA in PBS) overnight. Afterwards, a mouse monoclonal anti-collagen type I (1:100; Thermo Fisher scientific) or a mouse monoclonal anti-collagen type II (1:50; Novus Biologicals) were added and incubated overnight again at $4{ }^{\circ} \mathrm{C}$. The following day, samples were washed three times with wash buffer (blocking buffer without goat serum) and incubated with goat anti-mouse Alexa Fluor 647 secondary antibody (Invitrogen, USA) (1:300) in wash buffer for $1.5 \mathrm{~h}$ at RT. Cell nuclei were stained with DAPI $(0.1 \mu \mathrm{g} / \mathrm{mL}$ in PBS) for $5 \mathrm{~min}$, followed by washing in PBS (three times). Samples without primary antibodies were used as negative control. Fluorescent images were acquired using an inverted fluorescent microscope (Nikon Eclipse Ti-S).

\subsubsection{Glycosaminoglycan (GAG) assay}

GAG and DNA measurements were performed after 14 and 21 days of culture. Samples $(\mathrm{n}=4)$ were washed with PBS, transferred to a $1.5 \mathrm{~mL}$ Eppendorf tube and stored dry overnight at $-80^{\circ} \mathrm{C}$. Three freeze/thaw cycles (from $-80^{\circ} \mathrm{C}$ to room temperature) were applied to all the samples and $250 \mu \mathrm{L}$ of proteinase $\mathrm{K}$ digestion buffer(the formulation as describe above) were added to each tube, followed by incubation at $56^{\circ} \mathrm{C}$ for $16 \mathrm{~h}$, under agitation. The lysate was collected and used to measure the GAG amount and respective DNA content. The GAG amount was determined spectrophotometrically after reaction of $25 \mu \mathrm{L}$ of lysate mixed with $5 \mu \mathrm{L}$ of $\mathrm{NaCl}$ solution $(2.3 \mathrm{M})$ with $150 \mu \mathrm{L}$ of dimethylmethylene blue dye (DMMB, Sigma-Aldrich). The intensity of colour change was quantified immediately by measuring the absorbance at $525 \mathrm{~nm}$ in a microplate spectrophotometer (CLARIOstar, BMG Labtech, Germany). The GAG content was then calculated using a standard of chondroitin sulphate (Sigma-Aldrich). The DNA amount was determined following the protocol described above.

\subsubsection{Histology}

Pellets and cell-seeded scaffolds $(n=3)$ were retrieved after 21 days of culture, washed with PBS and fixed overnight in $10 \%$ neutral buffered formalin, at $4^{\circ} \mathrm{C}$. Subsequently, 
samples were dehydrated through a graded ethanol series $(1 \times 50,60,70,80,90$ and $96 \%$ and $2 \times 100 \%$ ), cleared with butanol $(2 \mathrm{~h}$ ), infiltrated with liquid paraffin (overnight), embedded in paraffin and stored at $-20^{\circ} \mathrm{C}$. Sections with $10 \mu \mathrm{m}$ in thickness were cut with a microtome (HM355S, Thermo Fisher Scientific), mounted in poly-L-lysine precoated slides and stained with $0.5 \%(\mathrm{w} / \mathrm{v})$ Alcian Blue solution in $1 \mathrm{M} \mathrm{HCl}(\mathrm{pH}=1.0$, Sigma-Aldrich) for sulphated GAG and with Nuclear Fast Red for cell visualization. Microphotographs were then acquired using an inverted fluorescent microscope (Nikon Eclipse Ti-S) equipped with a colour camera (DS-Ri2).

\subsubsection{Image analysis}

SEM images were analysed for fiber alignment quantification using the ImageJ software. The plugin OrientationJ was employed for this purpose and the images were analysed over the same ROI. Measurements $(n=30)$ were performed per condition and the results are expressed in terms of directional coherency coefficient, in a scale from zero to one. A coherency coefficient close to one, represented as a slender ellipse, indicates a strongly coherent orientation of the local fibers in the direction of the ellipse long axis. A coherency coefficient close to zero, represented geometrically as a circle, denotes no preferential orientation of the fibers[49].

\subsubsection{Statistics}

Statistical significance was assessed with an unpaired $t$ test on the fiber diameter study. A $p$-value $<0.05$ was considered significant. For the biological study, statistical significance was assessed using non-parametric tests. For comparison between two groups, the Mann-Whitney $U$ test was used and for comparison between three groups, the Kruskal-Wallis test was applied. A $p$-value $<0.05$ was considered significant. All the values are expressed as the average \pm standard deviation.

\section{Acknowledgements}

H. Chen thanks China Council Scholarship programme for financial support (Grant \# 2011614016) 


\section{References}

[1] Z. Izadifar, X. Chen, W. Kulyk, Strategic design and fabrication of engineered scaffolds for articular cartilage repair, Journal of functional biomaterials 3(4) (2012) 799-838.

[2] A.J. Sophia Fox, A. Bedi, S.A. Rodeo, The basic science of articular cartilage: structure, composition, and function, Sports health 1(6) (2009) 461-468.

[3] L. Zhang, J. Hu, K.A. Athanasiou, The role of tissue engineering in articular cartilage repair and regeneration, Critical Reviews ${ }^{\mathrm{TM}}$ in Biomedical Engineering 37(1-2) (2009).

[4] C. Mota, D. Puppi, F. Chiellini, E. Chiellini, Additive manufacturing techniques for the production of tissue engineering constructs, Journal of tissue engineering and regenerative medicine 9(3) (2015) 174190.

[5] X. Wang, B. Ding, B. Li, Biomimetic electrospun nanofibrous structures for tissue engineering, Materials Today 16(6) (2013) 229-241.

[6] D. Hutmacher, T. Woodfield, P. Dalton, J. Lewis, Scaffold design and fabrication, Tissue engineering (2008) 403-454.

[7] J. Lee, Y.H. Jeong, D.W. Cho, Fabrication of nanofibrous mats with uniform thickness and fiber density, Macromolecular Materials and Engineering 299(9) (2014) 1052-1061.

[8] B. Sun, Y. Long, H. Zhang, M. Li, J. Duvail, X. Jiang, H. Yin, Advances in three-dimensional nanofibrous macrostructures via electrospinning, Progress in Polymer Science 39(5) (2014) 862-890.

[9] N. Bhardwaj, S.C. Kundu, Electrospinning: a fascinating fiber fabrication technique, Biotechnology advances 28(3) (2010) 325-347.

[10] C.M. Rogers, G.E. Morris, T.W. Gould, R. Bail, S. Toumpaniari, H. Harrington, J.E. Dixon, K.M. Shakesheff, J. Segal, F.R. Rose, A novel technique for the production of electrospun scaffolds with tailored three-dimensional micro-patterns employing additive manufacturing, Biofabrication 6(3) (2014) 035003.

[11] S. Neubert, D. Pliszka, A. Góra, A. Jaworek, E. Wintermantel, S. Ramakrishna, Focused deposition of electrospun polymer fibers, Journal of Applied Polymer Science 125(1) (2012) 820-827.

[12] L.M. Bellan, H. Craighead, Control of an electrospinning jet using electric focusing and jet-steering fields, Journal of Vacuum Science \& Technology B: Microelectronics and Nanometer Structures Processing, Measurement, and Phenomena 24(6) (2006) 3179-3183.

[13] J. Lee, S.Y. Lee, J. Jang, Y.H. Jeong, D.-W. Cho, Fabrication of patterned nanofibrous mats using direct-write electrospinning, Langmuir 28(18) (2012) 7267-7275.

[14] J. Lee, J. Jang, H. Oh, Y.H. Jeong, D.-W. Cho, Fabrication of a three-dimensional nanofibrous scaffold with lattice pores using direct-write electrospinning, Materials Letters 93 (2013) 397-400.

[15] D. Sun, C. Chang, S. Li, L. Lin, Near-field electrospinning, Nano letters 6(4) (2006) 839-842.

[16] G. Zheng, W. Li, X. Wang, D. Wu, D. Sun, L. Lin, Precision deposition of a nanofibre by near-field electrospinning, Journal of Physics D: Applied Physics 43(41) (2010) 415501.

[17] C. Chang, K. Limkrailassiri, L. Lin, Continuous near-field electrospinning for large area deposition of orderly nanofiber patterns, Applied Physics Letters 93(12) (2008) 123111.

[18] T.D. Brown, P.D. Dalton, D.W. Hutmacher, Direct writing by way of melt electrospinning, Advanced Materials 23(47) (2011) 5651-5657. 
[19] T.D. Brown, P.D. Dalton, D.W. Hutmacher, Melt electrospinning today: An opportune time for an emerging polymer process, Progress in Polymer Science 56 (2016) 116-166.

[20] J. Visser, F.P. Melchels, J.E. Jeon, E.M. Van Bussel, L.S. Kimpton, H.M. Byrne, W.J. Dhert, P.D. Dalton, D.W. Hutmacher, J. Malda, Reinforcement of hydrogels using three-dimensionally printed microfibres, Nature communications 6 (2015).

[21] F. Chen, G. Hochleitner, T. Woodfield, J. Groll, P.D. Dalton, B.G. Amsden, Additive manufacturing of a photo-cross-linkable polymer via direct melt electrospinning writing for producing high strength structures, Biomacromolecules 17(1) (2015) 208-214.

[22] B.L. Farrugia, T.D. Brown, Z. Upton, D.W. Hutmacher, P.D. Dalton, T.R. Dargaville, Dermal fibroblast infiltration of poly ( $\varepsilon$-caprolactone) scaffolds fabricated by melt electrospinning in a direct writing mode, Biofabrication 5(2) (2013) 025001.

[23] N. Wright, J. Humphrey, Denaturation of collagen via heating: an irreversible rate process, Annual review of biomedical engineering 4(1) (2002) 109-128.

[24] N. Kishi, H. lizuka, The barus effect of polymer melts, Journal of Polymer Science Part C: Polymer Letters 2(4) (1964) 399-402.

[25] G. Hochleitner, T. Jüngst, T.D. Brown, K. Hahn, C. Moseke, F. Jakob, P.D. Dalton, J. Groll, Additive manufacturing of scaffolds with sub-micron filaments via melt electrospinning writing, Biofabrication $7(3)$ (2015) 035002.

[26] N. Abdul Rahman, Electrospun Conducting Polymer Nanofibers for Biomedical Applications, ResearchSpace@ Auckland, 2012.

[27] G. Beumer, C. Van Blitterswijk, D. Bakker, M. Ponec, Cell-seeding and in vitro biocompatibility evaluation of polymeric matrices of PEO/PBT copolymers and PLLA, Biomaterials 14(8) (1993) 598-604.

[28] A. Radder, H. Leenders, C. Van Blitterswijk, Application of porous PEO/PBT copolymers for bone replacement, Journal of Biomedical Materials Research Part A 30(3) (1996) 341-351.

[29] Z. Li, C. Wang, One-dimensional nanostructures: electrospinning technique and unique nanofibers, Springer2013.

[30] P. Heikkilä, A. Harlin, Electrospinning of polyacrylonitrile (PAN) solution: Effect of conductive additive and filler on the process, Express Polymer Letters 3(7) (2009) 437-445.

[31] K. Garg, G.L. Bowlin, Electrospinning jets and nanofibrous structures, Biomicrofluidics 5(1) (2011) 013403.

[32] D.H. Reneker, A.L. Yarin, Electrospinning jets and polymer nanofibers, Polymer 49(10) (2008) 23872425.

[33] S. De Vrieze, T. Van Camp, A. Nelvig, B. Hagström, P. Westbroek, K. De Clerck, The effect of temperature and humidity on electrospinning, Journal of materials science 44(5) (2009) 1357.

[34] A. Di Luca, I. Lorenzo-Moldero, C. Mota, A. Lepedda, D. Auhl, C. Van Blitterswijk, L. Moroni, Tuning Cell Differentiation into a 3D Scaffold Presenting a Pore Shape Gradient for Osteochondral Regeneration, Advanced healthcare materials 5(14) (2016) 1753-1763.

[35] A. Di Luca, K. Szlazak, I. Lorenzo-Moldero, C.A. Ghebes, A. Lepedda, W. Swieszkowski, C. Van Blitterswijk, L. Moroni, Influencing chondrogenic differentiation of human mesenchymal stromal cells in scaffolds displaying a structural gradient in pore size, Acta biomaterialia 36 (2016) 210-219. 
[36] M.M. Nava, L. Draghi, C. Giordano, R. Pietrabissa, The effect of scaffold pore size in cartilage tissue engineering, Journal of Applied Biomaterials \& Functional Materials 14(3) (2016).

[37] J. Xu, M. Abecassis, Z. Zhang, P. Guo, J. Huang, K. Ehmann, J. Cao, Accuracy Improvement of Nanofiber Deposition by Near-Field Electrospinning.

[38] X. Quan, Y. Ji, H. Zhang, Y. Zhang, X. Xu, T. Zhong, Charging compensation of alumina samples by using an oxygen microinjector in the environmental scanning electron microscope, Scanning 28(5) (2006) 289-293.

[39] Y. Ji, H. Guo, T. Zhong, H. Zhang, X. Quan, Y. Zhang, X. Xu, Charge and charging compensation on oxides and hydroxides in oxygen environmental SEM, Ultramicroscopy 103(3) (2005) 191-198.

[40] J. Steele, S. McCullen, A. Callanan, H. Autefage, M. Accardi, D. Dini, M. Stevens, Combinatorial scaffold morphologies for zonal articular cartilage engineering, Acta biomaterialia 10(5) (2014) 2065-2075.

[41] S.-M. Lien, L.-Y. Ko, T.-J. Huang, Effect of pore size on ECM secretion and cell growth in gelatin scaffold for articular cartilage tissue engineering, Acta Biomaterialia 5(2) (2009) 670-679.

[42] K.A. Athanasiou, M. Rosenwasser, J. Buckwalter, T. Malinin, V. Mow, Interspecies comparisons of in situ intrinsic mechanical properties of distal femoral cartilage, Journal of Orthopaedic Research 9(3) (1991) 330-340.

[43] J.M. Mansour, Biomechanics of cartilage, Kinesiology: the mechanics and pathomechanics of human movement (2003) 66-79.

[44] X. Zhang, D. Blalock, J. Wang, Classifications and Definitions of Normal Joints, 2015.

[45] N.V. Ghone, W.L. Grayson, Recapitulation of mesenchymal condensation enhances in vitro chondrogenesis of human mesenchymal stem cells, Journal of cellular physiology 227(11) (2012) 3701 3708 .

[46] B. Cao, Z. Li, R. Peng, J. Ding, Effects of cell-cell contact and oxygen tension on chondrogenic differentiation of stem cells, Biomaterials 64 (2015) 21-32.

[47] J.S. Park, J.S. Chu, A.D. Tsou, R. Diop, Z. Tang, A. Wang, S. Li, The effect of matrix stiffness on the differentiation of mesenchymal stem cells in response to TGF- $\beta$, Biomaterials 32(16) (2011) 3921-3930.

[48] Y. Minegishi, K. Hosokawa, N. Tsumaki, Time-lapse observation of the dedifferentiation process in mouse chondrocytes using chondrocyte-specific reporters, Osteoarthritis and Cartilage 21(12) (2013) 19681975.

[49] T. Hui, K. Cheung, W. Cheung, D. Chan, B. Chan, In vitro chondrogenic differentiation of human mesenchymal stem cells in collagen microspheres: influence of cell seeding density and collagen concentration, Biomaterials 29(22) (2008) 3201-3212.

[50] S. Kobayashi, A. Meir, J. Urban, Effect of cell density on the rate of glycosaminoglycan accumulation by disc and cartilage cells in vitro, Journal of Orthopaedic Research 26(4) (2008) 493-503.

[51] C. Kiani, C. Liwen, Y.J. Wu, J.Y. Albert, B.Y. Burton, Structure and function of aggrecan, Cell research 12(1) (2002) 19-32.

[52] H. Akiyama, Control of chondrogenesis by the transcription factor Sox9, Modern Rheumatology 18(3) (2008) 213-219. 
[53] I. Sekiya, K. Tsuji, P. Koopman, H. Watanabe, Y. Yamada, K. Shinomiya, A. Nifuji, M. Noda, SOX9 enhances aggrecan gene promoter/enhancer activity and is up-regulated by retinoic acid in a cartilagederived cell line, TC6, Journal of Biological Chemistry 275(15) (2000) 10738-10744.

[54] T. Aigner, P.M. Gebhard, E. Schmid, B. Bau, V. Harley, E. Pöschl, SOX9 expression does not correlate with type II collagen expression in adult articular chondrocytes, Matrix Biology 22(4) (2003) 363-372.

[55] J. Sohier, T. Vlugt, N. Cabrol, C. Van Blitterswijk, K. De Groot, J. Bezemer, Dual release of proteins from porous polymeric scaffolds, Journal of Controlled Release 111(1) (2006) 95-106.

[56] C.M. DiGirolamo, D. Stokes, D. Colter, D.G. Phinney, R. Class, D.J. Prockop, Propagation and senescence of human marrow stromal cells in culture: a simple colony-forming assay identifies samples with the greatest potential to propagate and differentiate, British Journal of Haematology 107(2) (1999) 275281.

[57] D.G. Phinney, G. Kopen, W. Righter, S. Webster, N. Tremain, D.J. Prockop, Donor variation in the growth properties and osteogenic potential of human marrow stromal cells, Journal of cellular biochemistry 75(3) (1999) 424-436. 


\section{Supplementary Information}

\section{Materials and Methods: Polymer Solution Characterization}

\subsection{Viscosity}

The viscosity was measured using a viscometer (Brookfield DV-E, Brookfield Engineering). Solution ( $8 \mathrm{~mL})$ was loaded into the device's container and a cylindrical spindle was used and set to 30,50 , and $100 \mathrm{rpm}$. Three different measurements were taken for each rotational speed and the final viscosity value was averaged from these results.

\subsection{Surface Tension}

The evaluation of the polymers solution's surface tension was carried out using an optical contact angle measuring system (OCA 20, Dataphysics) at $25^{\circ} \mathrm{C}$. Since the organic solvents in the solution have high evaporation rates, the preferred method for surface tension measuring was an axisymmetric drop shape analysis (ADSA) of an air bubble/polymer solution interface. ADSA is a powerful tool for fast, accurate and reliable measurements of fluid-liquid interfacial tensions. ADSA is based on the numerical fit between the shape of an experimental bubble (or drop) and the mathematical model given by the classical Laplace equation of capillarity[58]. In this procedure, a closed container was filled with the polymer solution and a capillary (with the tip bent upwards) was dipped in it. An air bubble was precisely dosed through the capillary and the evolution of the drop shape and contact angle was automatically analysed and used to extrapolate the surface tension. The surface tension of deionized (DI) water at $25^{\circ} \mathrm{C}$ $(\gamma=71.99 \pm 0.005)$ was used as an accuracy control.

\subsection{Conductivity}

Conductivity measurements were obtained using an impedance spectroscopy-based method. Impedance spectroscopy provides the relation between the real $(\mathrm{Zr})$ and imaginary $(\mathrm{Zi})$ impedance of a sample, as a function of the applied frequency, and the results can then be graphically expressed in the Nyquist plot, as Zr v. Zi. The impedance of a resistance is independent of the frequency, and thus it has no imaginary part [25]. Hence, it is possible to determine the resistance of a solution from a Nyquist plot by fitting 
the resulted curve and calculating the impedance when the imaginary part is zero. Once determining the resistance $(R)$, the electrical resistivity of a solution $(\rho)$ can be determined using the relation $R=\rho(L / A)$, where $L$ is the distance between the electrodes and $A$ is the area of the electrode. To determine these parameters precisely, solutions of known conductivity $(\sigma)$ can be used to calibrate the system, since $\sigma=1 / \rho$. Potassium chloride $(\mathrm{KCl})$ solutions of $1 \mathrm{M}, 0.1 \mathrm{M}, 0.01 \mathrm{M}, 0.001 \mathrm{M}$ and $0.0001 \mathrm{M}$ were then prepared and the resistance measured. The results were fitted into a curve and the calibration factor of the system (L/A) could then be determined. Finally, to obtain the polymer solution conductivity, the resistance was first measured through the Nyquist plot fit and the conductivity calculated using the calibration factor. The impedance spectroscopy measurements were carried using the Autolab software (Metrohm Autolab B.V.) and using a setup built in-house.

\section{Figures}

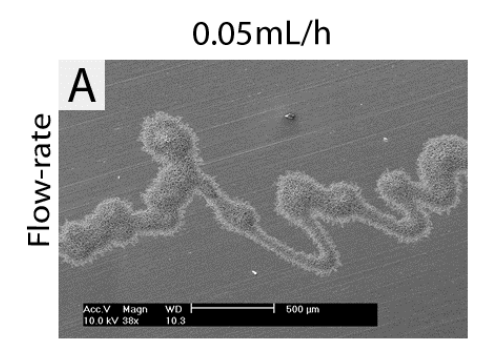

$8 \mathrm{kV}$

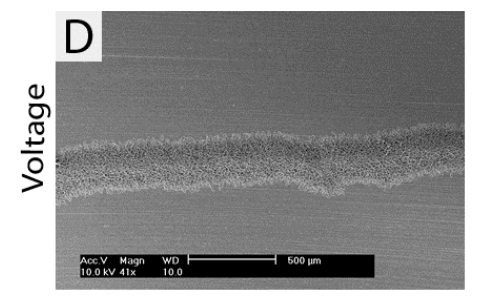

$8 \mathrm{~cm}$

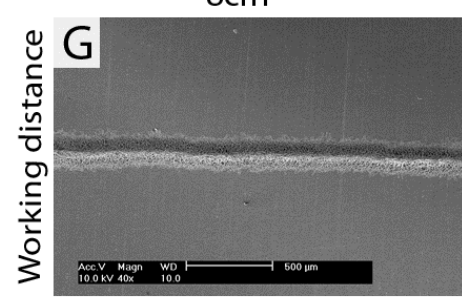

$0.1 \mathrm{~mL} / \mathrm{h}$

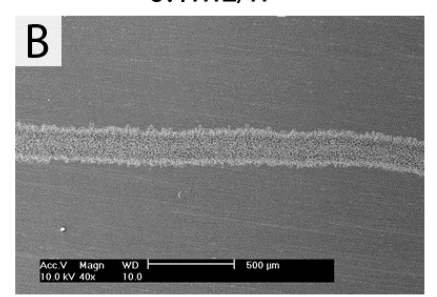

$12 \mathrm{kV}$

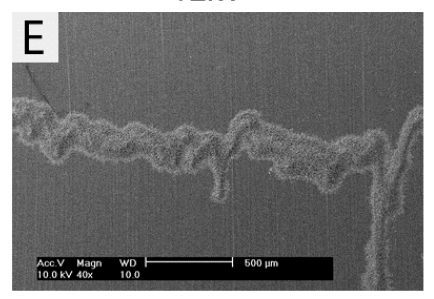

$10 \mathrm{~cm}$

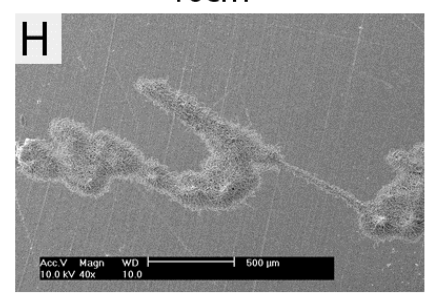

$0.5 \mathrm{~mL} / \mathrm{h}$

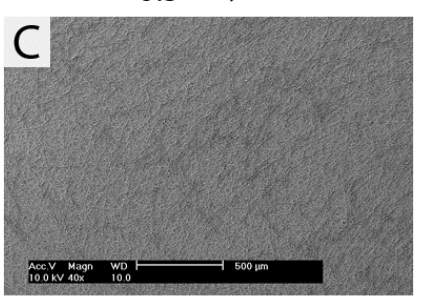

$16 \mathrm{kV}$

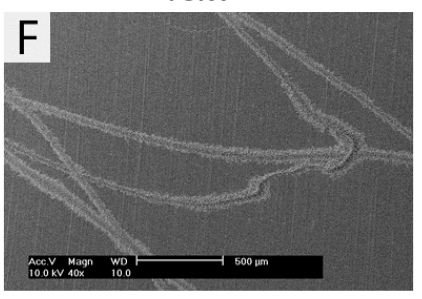

$12 \mathrm{~cm}$

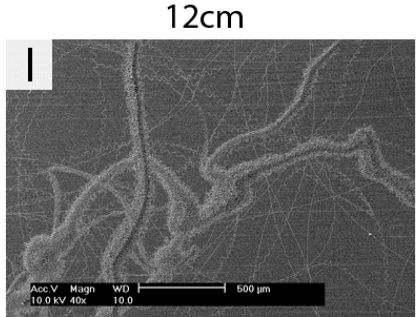

Figure S1. Influence of the processing parameters on the deposited line consistency. This gross examination was used to identify the direct writing region. (A-C) Effect of different flow rates at a voltage $(\mathrm{V})$ is $8 \mathrm{kV}$ and working distance $(\mathrm{Wd})$ of $8 \mathrm{~cm}$; $(\mathrm{D}-\mathrm{F})$ Effect of the voltages at $\mathrm{Wd}=8 \mathrm{~cm}$ and flow-rate is $0.08 \mathrm{~mL} / \mathrm{h}$; (G-I) Effect of different working distance at $\mathrm{V}=8 \mathrm{kV}$ and the flow-rate is $0.08 \mathrm{~mL} / \mathrm{h}$ Scale bars: $A-$ I $(500 \mu \mathrm{m})$. 

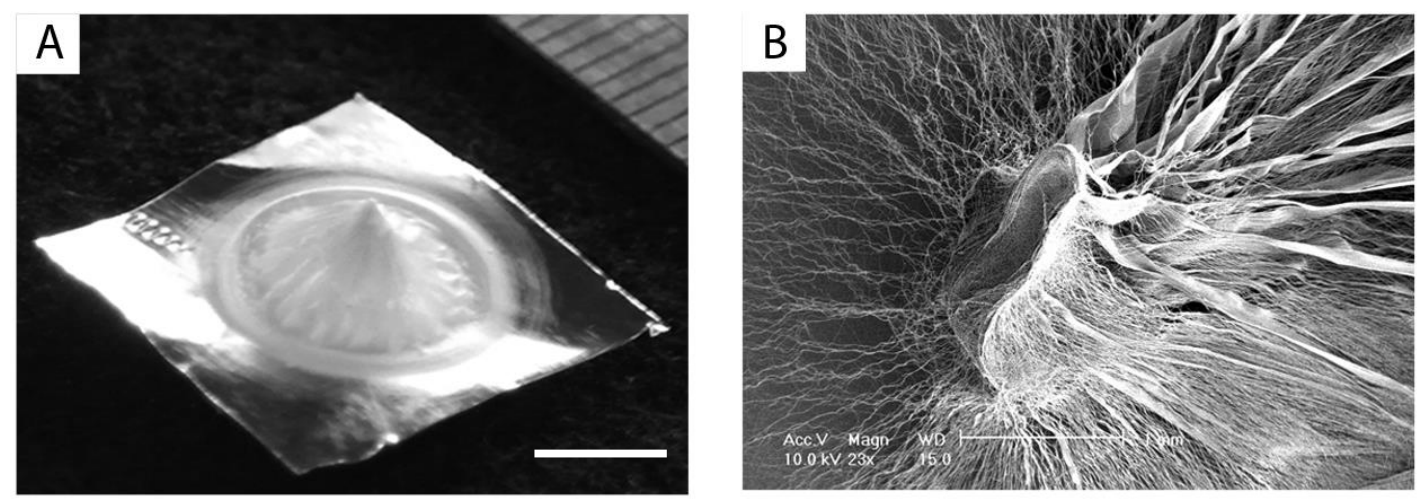

Figure S2. Point pattern created when the solution is let to spin at a single point for a prolonged period. (A) Digital camera image; (B) SEM micrograph. Scale bars: A $(5 \mathrm{~mm})$ and B $(1 \mathrm{~mm})$.

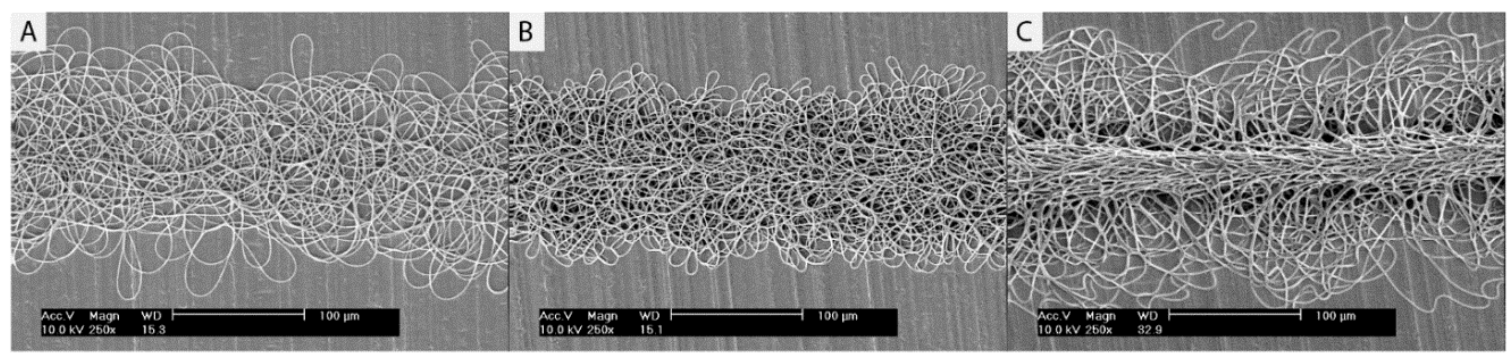

Figure S3. Bundle morphology resulting from different voltages: (A) $4 \mathrm{kV}$, (B) $5 \mathrm{kV}$ and (C) $6 \mathrm{kV}$. The working distance was maintained at $5 \mathrm{~cm}$, the flow-rate at $0.18 \mathrm{~mL} / \mathrm{h}$ and the scan-speed at $10 \mathrm{~mm} / \mathrm{s}$. Scale bars: A-C $(100 \mu \mathrm{m})$.

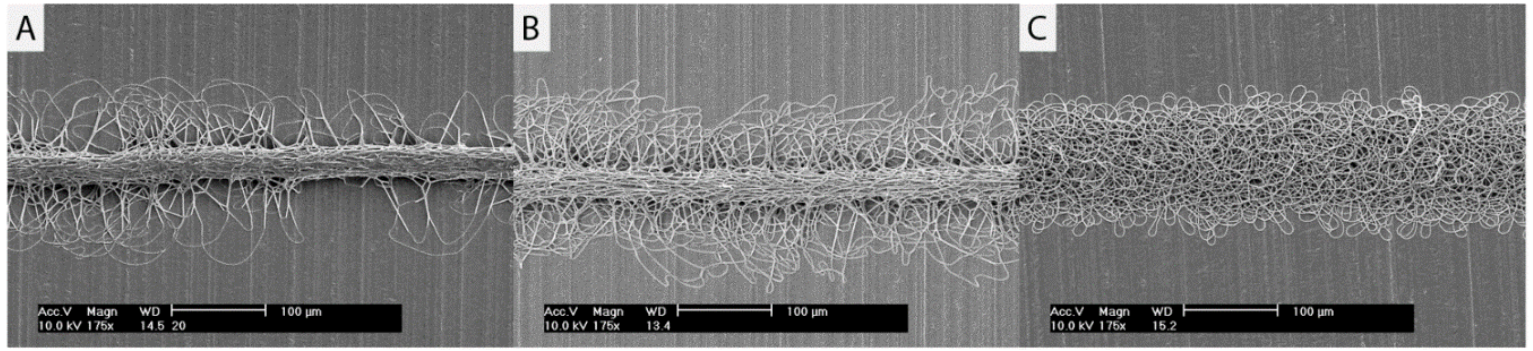

Figure S4. SEM images showing the effect of working distance on the deposited fibers at $(A) W d=3 \mathrm{~cm}$, (B) $W d=4 \mathrm{~cm}$ and (C) $W d=5 \mathrm{~cm}$. The applied voltage was set at $5 \mathrm{kV}$, the flow-rate at $0.18 \mathrm{~mL} / \mathrm{h}$ and the scan speed at $10 \mathrm{~mm} / \mathrm{s}$. Scale bars: A-C $(100 \mu \mathrm{m})$. 


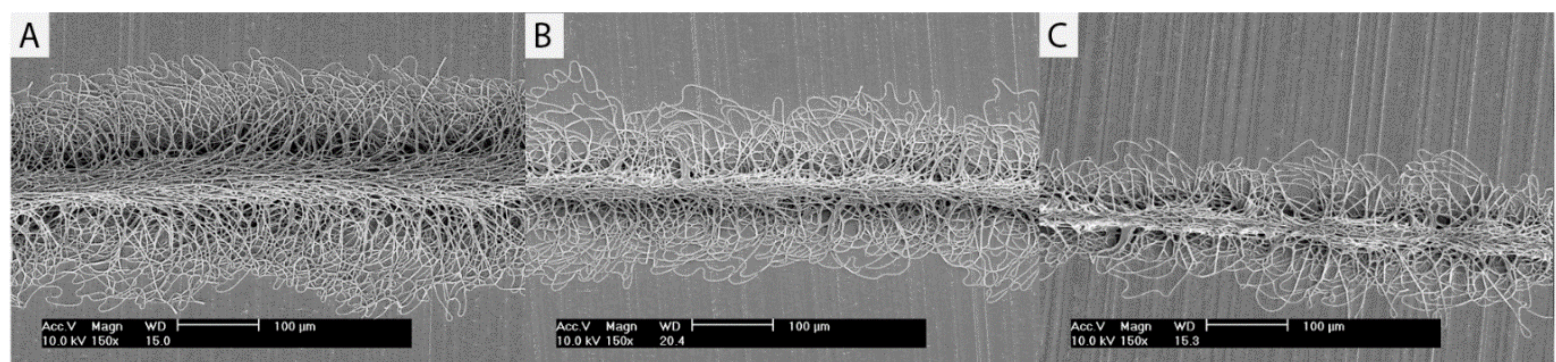

Figure S5. Effect on the line pattern at different scan speeds (A) $4 \mathrm{~mm} / \mathrm{s}$, (B) $7 \mathrm{~mm} / \mathrm{s}$ and (C) $10 \mathrm{~mm} / \mathrm{s}$. The voltage was set at $5 \mathrm{kV}$, the working distance at $5 \mathrm{~cm}$ and the flow-rate at $0.18 \mathrm{~mL} / \mathrm{h}$. Scale bars: $\mathrm{A}-\mathrm{C}(100$ $\mu \mathrm{m})$.
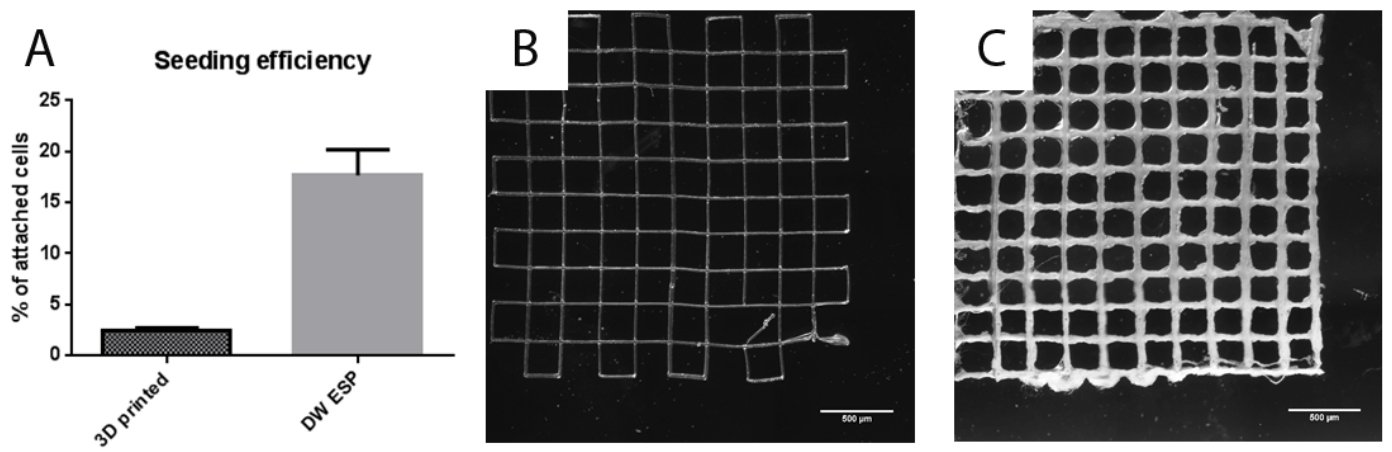

Figure S6. (A) Seeding efficiency of human mesenchymal stromal cells (hMSCs) on (B) 3D printed scaffolds and (C) direct-written (DW) electrospun (ESP) scaffolds. Efficiency measured as attached cells relative to total cells seeded on scaffolds and expressed as mean \pm SD. Scale bars: B-C (500 $\mu \mathrm{m})$. 

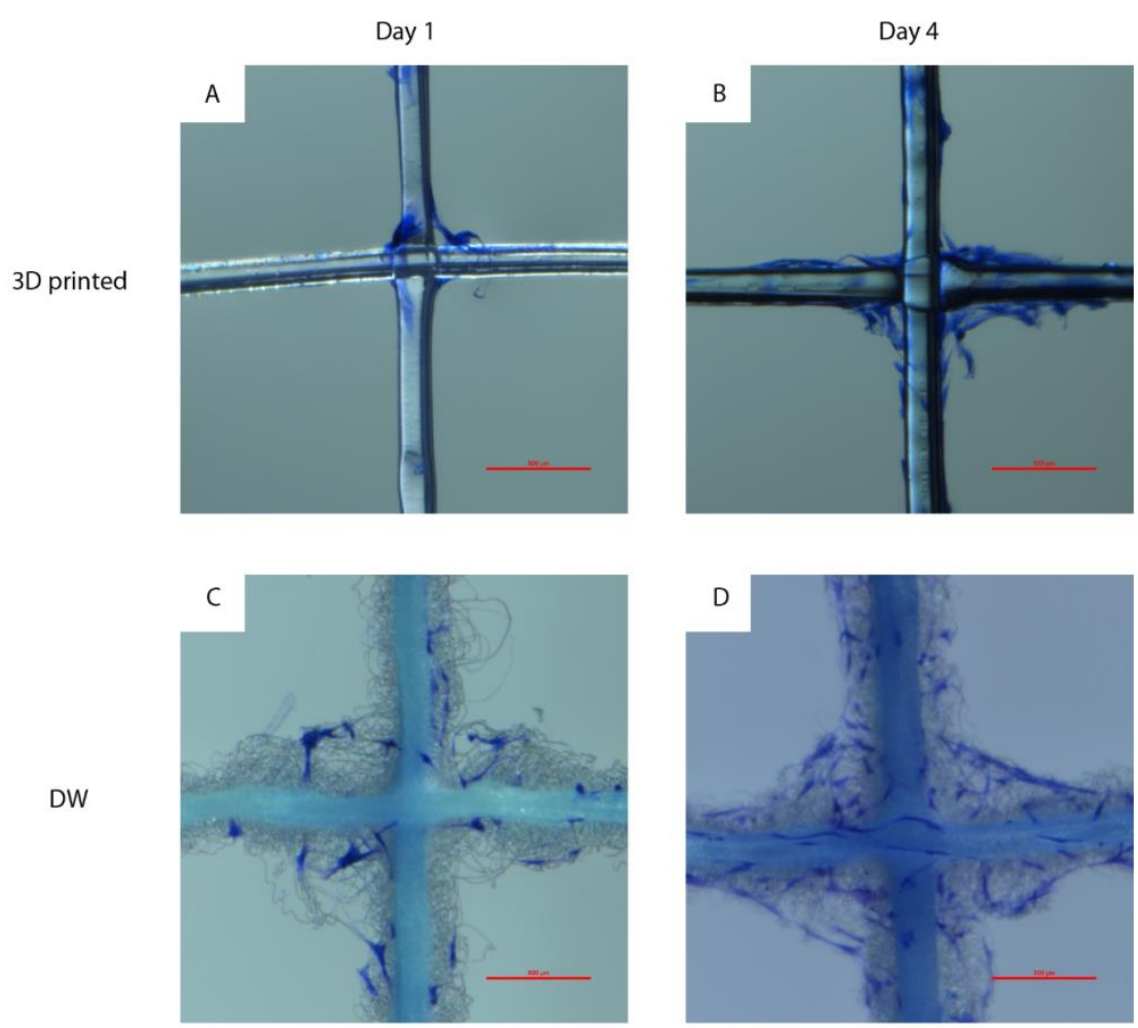

Figure S7. Methylene Blue staining on (A and B) 3D-printed and (C and D) DW ESP scaffolds after (A and C) 1 day and ( $B$ and $D) 4$ days of culture in basic medium. Both scaffolds have the same pore space of 860 $\mu \mathrm{m}$ and an average line width of $150 \mu \mathrm{m}$, although the DW scaffolds appear larger due to the fiber protrusions. Scale bars represent $500 \mu \mathrm{m}$.

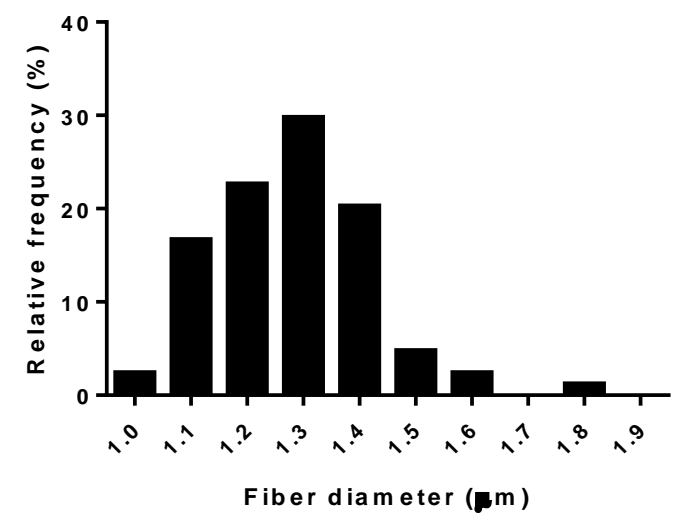

Figure S8. Frequency distribution of individual fiber diameters from the articular cartilage mimetic scaffold. 


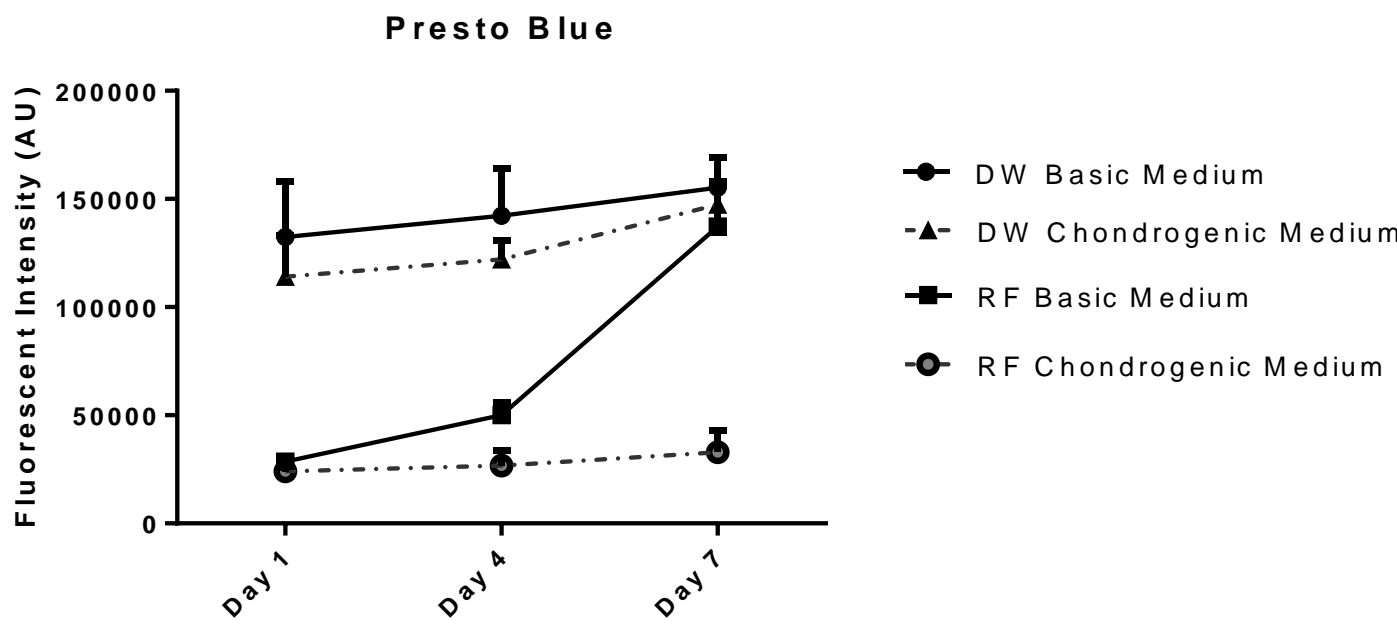

Figure S9. Presto blue measurements of the DW and RF seeded scaffolds taken at 3 times points during the first week of culture.
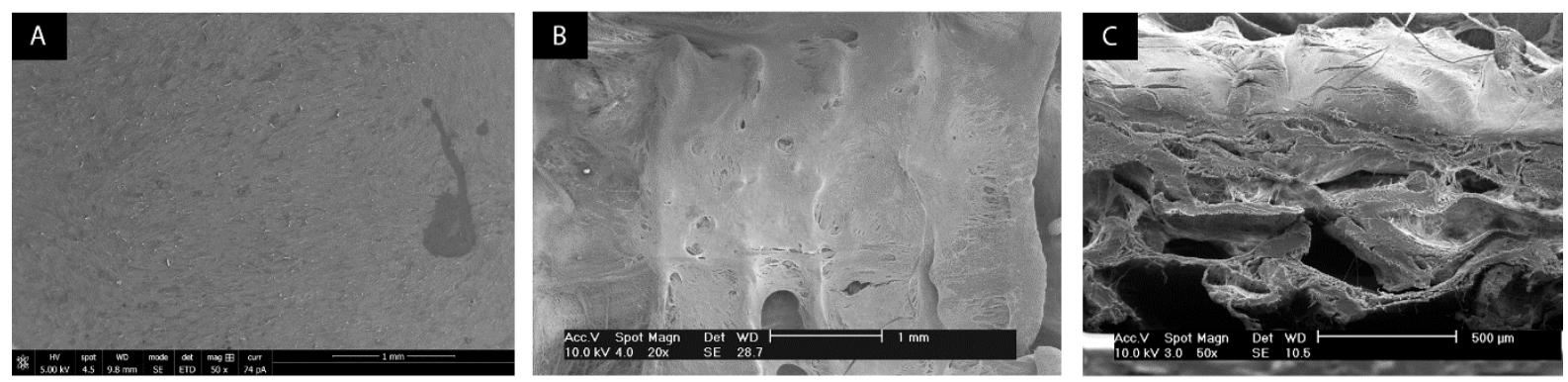

Figure S10. SEM micrographs of the cell-seeded scaffolds RF (A) and DW (B, C) after 21 days in culture on chondrogenic medium. (C) cross-section of the DW scaffold shows cells and matrix grow insides the scaffolds. Scale bars: A and B (1 mm), and C $(500 \mu \mathrm{m})$.

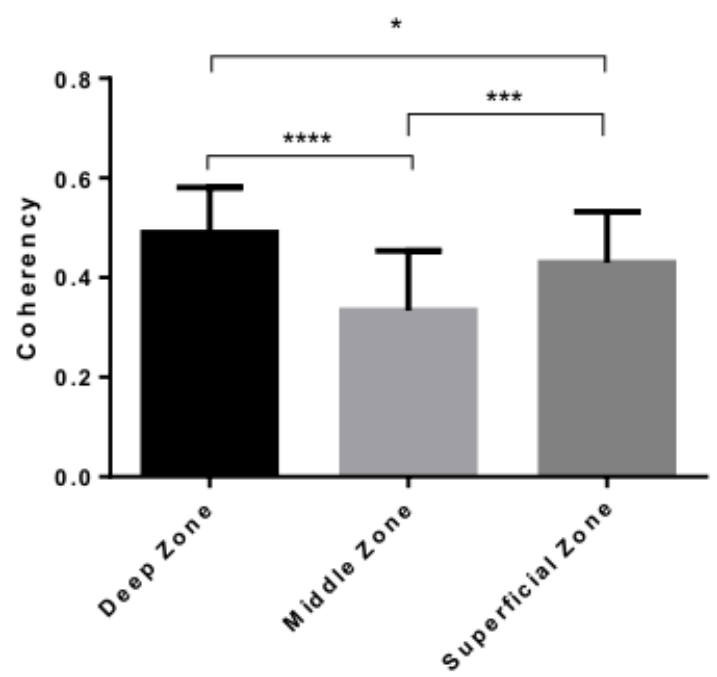

Figure S11. Directional coherency of the fibril matrix in the different sections of the DW ESP scaffold. 0 no coherency; 1 - full coherency (full alignment). ${ }^{*} p<0.05 ;{ }^{* * *} p<0.001$. 

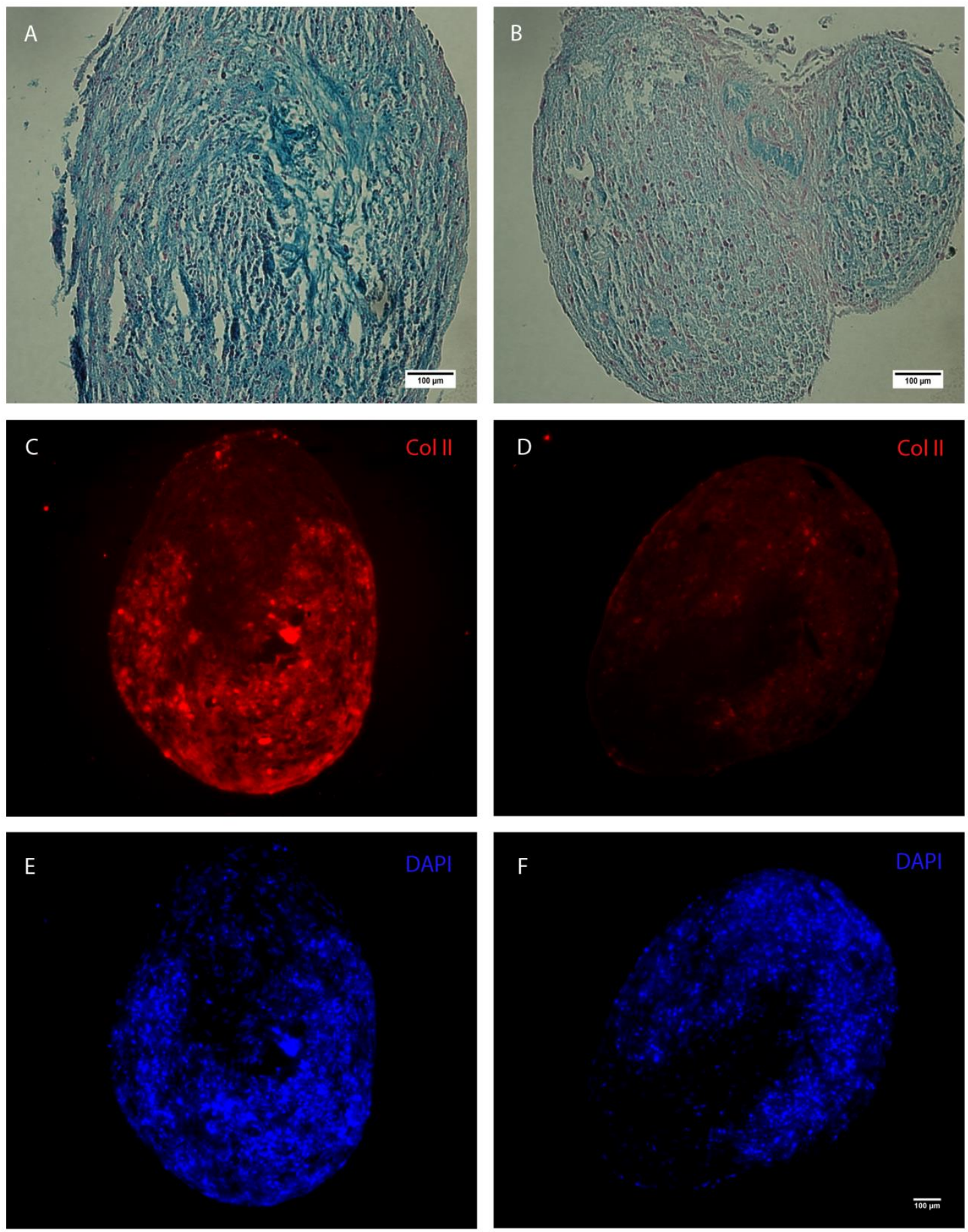

Figure S12. Chondrogenic differentiation of the hMSCs cultured in a pellet form in differentiation (left column) or control (right column) medium for 21 days. ( $A$ and $B$ ) Alcian Blue staining; ( $C$ and $D$ ) Collagen type II immunostaining; (E and F) DAPI staining. Scale bars: A-F $(100 \mu \mathrm{m})$; scale bar in F applies to C-E panels. 

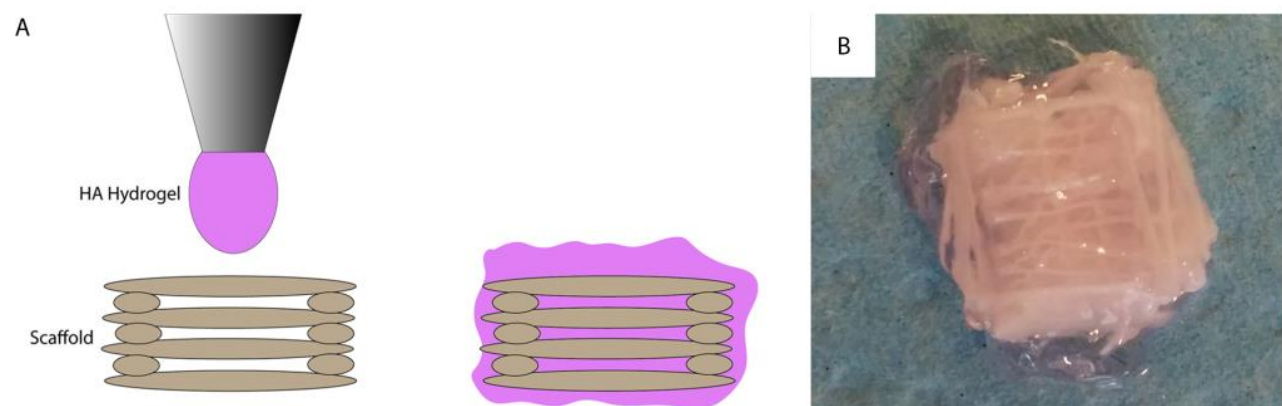

Figure S13. (A) Schematic of the process of scaffold embedding with a hyaluronic acid (HA) hydrogel and (B) photograph of such construct. Scale bars: $1 \mathrm{~cm}$.

Basic Medium
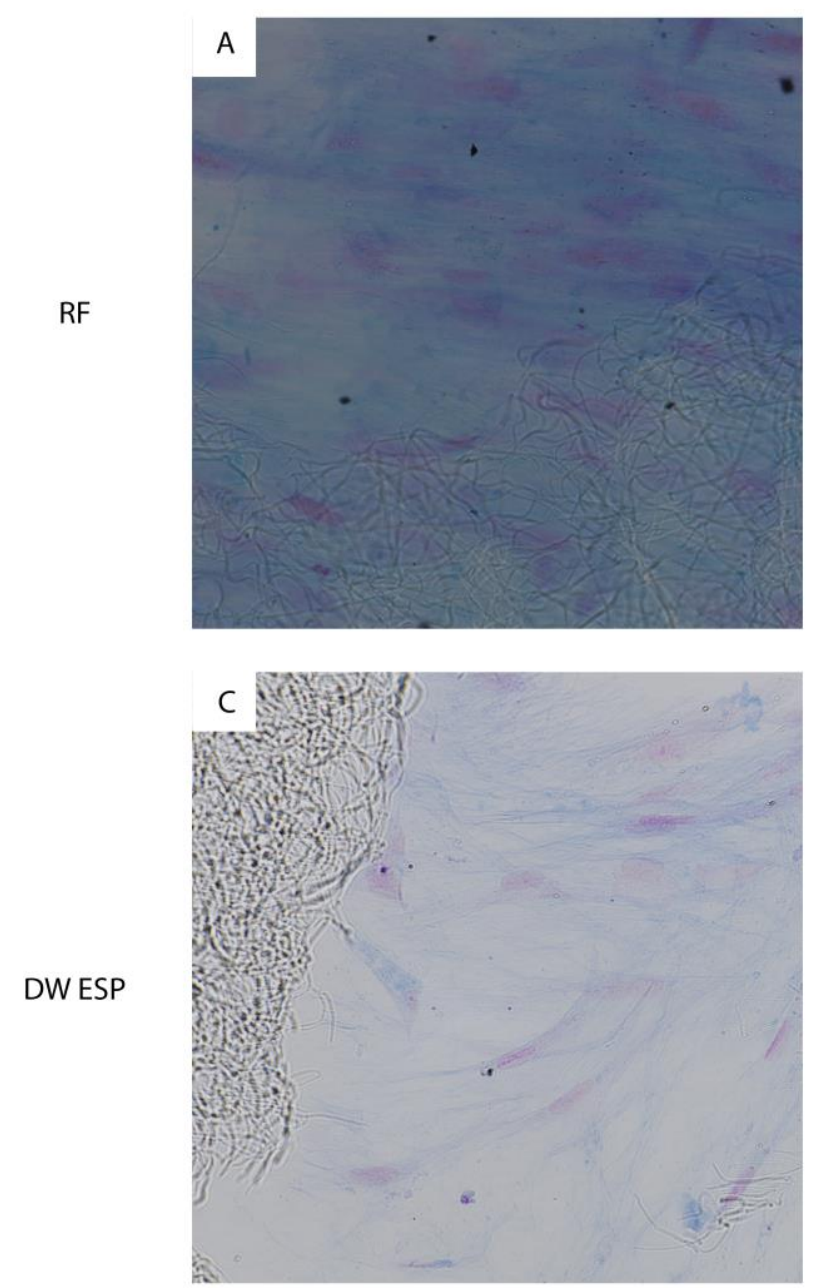

Chondrogenic medium
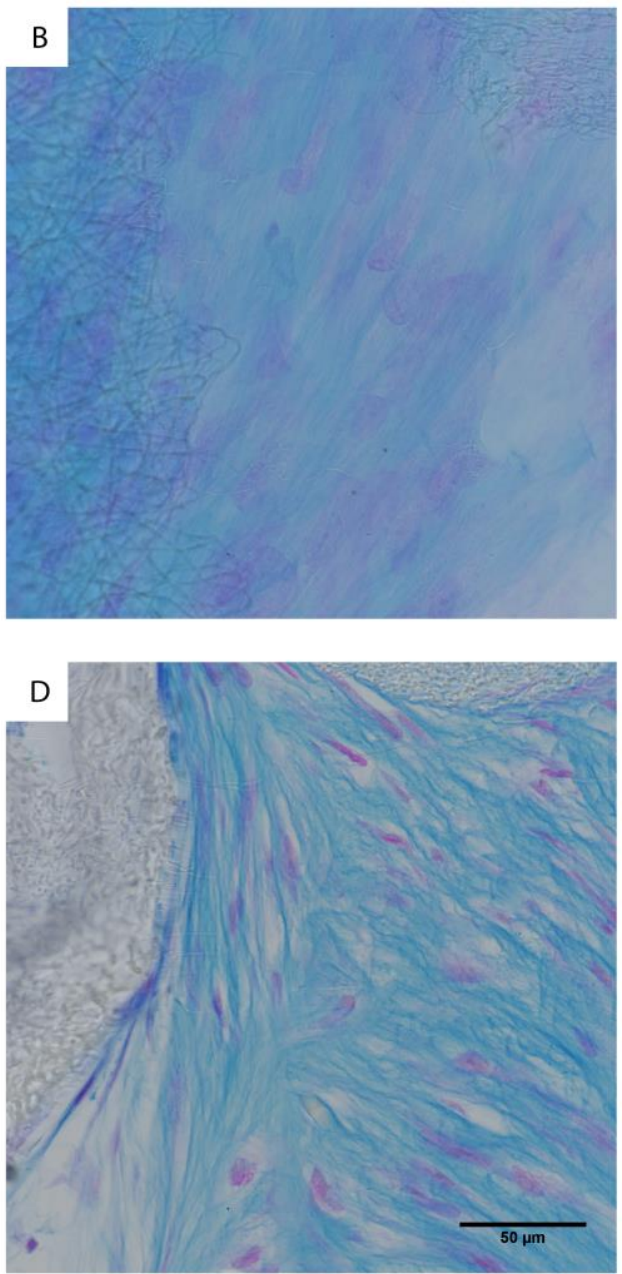

Figure S14. Alcian Blue and Nuclear Fast Red staining of histological sections of $\operatorname{RF}(A$ and $B)$ and $D W$ ESP ( $C$ and $D)$ scaffolds after 21 days culture in basic ( $A$ and $C$ ) and chondrogenic medium( $B$ and $D)$. Scale bar in $\mathrm{D}$ represents $50 \mu \mathrm{m}$ and applies to all panels. 
A

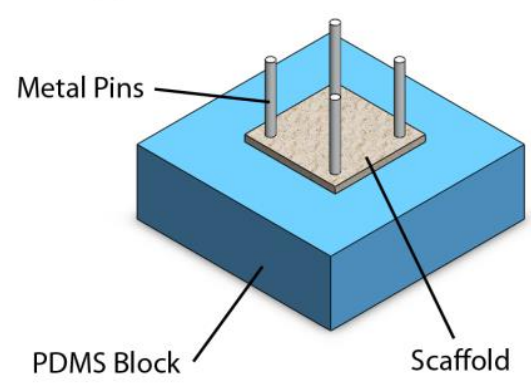

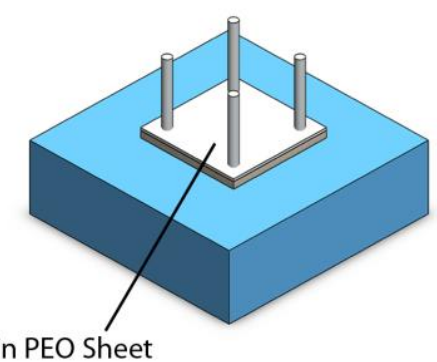

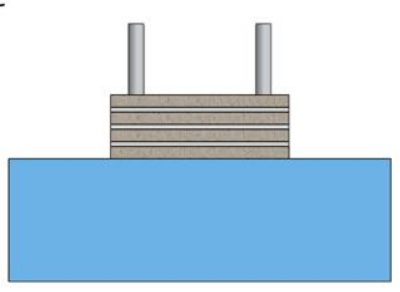

Figure S15. Scaffold mounting process: (A) The scaffold is placed on top of a PDMS block and fixed with thin metal bars; (B) a thin PEO sheet is then placed directly on top of the scaffold; (C) the process is repeated until 4 mats are assembled. The scaffold vertexes are then joined together and the sacrificial layers are dissolved in DI water.

\section{Tables}

Table S1. Summary of some physical properties from a solution of 28\% PEO55PBT45 dissolved in 75:25 Cholorform:HFIP.

\begin{tabular}{lc}
\hline Physical property & Value \\
\hline Viscosity $(\mathrm{cP})$ & $326.2 \pm 2.9$ \\
\hline Conductivity $(\boldsymbol{\mu S} / \mathrm{cm})$ & $4.7 \pm 0.2$ \\
\hline Surface Tension $(\mathrm{mN} / \mathrm{m})$ & $28.0 \pm 0.7$ \\
\hline
\end{tabular}


Table S2. Summary of the obtained fiber morphologies with varying working distances and voltages. The values in bold on the voltage column represent the minimum voltage for jet initiation. Once the jet starts to spin, the applied voltage can be reduced.

\begin{tabular}{cccc}
\hline Working Distance $(\mathbf{c m})$ & Voltage (kV) & Focused spinning? & Morphology \\
\hline \multirow{4}{*}{2} & 2 & $\mathrm{~N} / \mathrm{A}$ & $\mathrm{N} / \mathrm{A}$ \\
& 2.5 & + & $\mathrm{RF}$ \\
& 3 & + & $\mathrm{RF}$ \\
& 3.5 & + & $\mathrm{RF}$ \\
& 4 & + & $\mathrm{RF} / \mathrm{AC}$ \\
& 4.5 & + & $\mathrm{SF}$ \\
& 5 & - & - \\
\hline \multirow{3}{*}{3} & 3 & $\mathrm{~N} / \mathrm{A}$ & $\mathrm{N} / \mathrm{A}$ \\
& 3.5 & + & $\mathrm{RF}$ \\
& 4 & + & $\mathrm{RF}$ \\
& 4.5 & + & $\mathrm{RF}$ \\
& 5 & + & $\mathrm{RF}$ \\
& 5.5 & + & $\mathrm{RF} / \mathrm{AC}$ \\
& 6 & + & $\mathrm{AC}$ \\
& 7 & - & - \\
\hline & 3.5 & $\mathrm{~N} / \mathrm{A}$ & $\mathrm{N} / \mathrm{A}$ \\
& 4 & + & $\mathrm{RF}$ \\
& 4.5 & + & $\mathrm{RF}$ \\
& 5 & + & $\mathrm{RF}$ \\
& 5.5 & + & $\mathrm{AC}$ \\
& 6 & - & - \\
\hline
\end{tabular}

$N / A$, no spinning, no fiber

+ , yes

,- no
$\mathrm{RF}$, Bundle of random fibers

AC, Bundle of fibers with aligned core

$\mathrm{SF}$, Single fiber

Table S3. List of processing parameters that permitted DW ESP of PEOT/PBT.

\begin{tabular}{llc}
\hline \multicolumn{1}{c}{ Parameters } & Range \\
\hline - & Polymer Solution & \\
- & Concentration $(\mathrm{w} / \mathrm{v}, \%)$ & $27-29$ \\
$\mathbf{C}: \mathrm{HFIP}$ ratio $(\mathrm{v} / \mathrm{v})$ & $74: 26-78: 22$ \\
\hline & Process & \\
- & Working distance $(\mathrm{cm})$ & $\leq 8$ \\
- & Voltage $(\mathrm{kV})$ & $2.5 \leq \mathrm{V} \leq 8$ \\
- & Flow-rate $(\mathrm{mL} / \mathrm{h})$ & $0.08 \leq \mathrm{Q} \leq 0.3$ \\
- & Scan-speed $(\mathrm{mm} / \mathrm{s})$ & $\geq 5$ \\
\hline & Ambient & \\
- & Temperature $\left({ }^{\circ} \mathrm{C}\right)$ & $18.5-21$ \\
\hline
\end{tabular}


Table S4. Characterization of the articular cartilage mimetic scaffold

\begin{tabular}{ll}
\hline \multicolumn{1}{c}{ Features } & Value \\
\hline Individual scaffold thickness $(\mu \mathrm{m})$ & $208.5 \pm 19.4$ \\
Mounted scaffold thickness $(\mathrm{mm})$ & $1.39 \pm 0.6$ \\
\hline Fiber diameter $(\mu \mathrm{m})$ & $1.39 \pm 0.6$ \\
\hline Pore Space Superficial zone $(\mu \mathrm{m})$ & $238.0 \pm 79.3$ \\
\hline Pore Space Deep zone $(\mu \mathrm{m})$ & $523.6 \pm 156.2$ \\
\hline Porosity $(\%)$ & $91.2 \pm 1.0$ \\
\hline Young's Modulus in compression $(\mathrm{MPa})$ & $2.65 \pm 0.65$ \\
\hline
\end{tabular}

Table S5. List of ESP parameters used for scaffold fabrication.

\begin{tabular}{lcc}
\hline Parameter & DW scaffold & RF sheet \\
\hline Voltage (kV) & 5 & 9 \\
\hline Working distance (cm) & 3 & 4 \\
\hline Flow-rate $(\mathbf{m L} / \mathbf{h})$ & 0.12 & 0.4 \\
\hline Scan-speed $(\mathbf{m m} / \mathbf{s})$ & $35-50$ & 5 \\
\hline Spinneret diameter $(\mathbf{m m})$ & 0.5 & 0.5 \\
\hline Temperature $\left({ }^{\circ} \mathbf{C}\right)$ & $18-21$ & $30-32$ \\
\hline Relative Humidity (\%) & $38-43$ & 30
\end{tabular}

Table S6. List of qPCR primers sequence.

\begin{tabular}{lll}
\hline Gene & Forward & Reverse \\
\hline B2M & 5'-ACAAAGTCACATGGTTCACA & 5'-GACTTGTCTTTCAGCAAGGA \\
\hline Col2a & 5'-CGTCCAGATGACCTTCCTACG & 5'-TGAGCAGGGCCTTCTTGAG \\
\hline Sox9 & 5'-TGGGCAAGCTCTGGAGACTTC & 5'-ATCCGGGTGGTCCTTCTTGTG \\
\hline ACAN & 5'-AGGCAGCGTGATCCTTACC & 5'-GGCCTCTCCAGTCTCATTCTC \\
\hline ALCAM & 5'-ACGATGAGGCAGACGAGATAAGT & 5'-CAGCAAGGAGGAGACCAACAAC \\
\hline
\end{tabular}




\section{Bibliography}

[1] O. Del Rı, A. Neumann, Axisymmetric drop shape analysis: computational methods for the measurement of interfacial properties from the shape and dimensions of pendant and sessile drops, Journal of colloid and interface science 196(2) (1997) 136-147. 


\section{Chapter 7}

\section{General Discussion}

Despite it was created in the middle of 1990s, the field of regenerative medicine is expanding fast [1]. Whereas regenerative medicine first aimed at developing biological constructs that restore damaged or diseased tissues and organs [1-3], it is more and more progressing towards a field where the engineered biological constructs could potentially enhance tissues and organs. To this end, stem cells are often seeded onto an artificial scaffold, which is designed to facilitate cell reorganization and facilitate cell differentiation to form a functional tissue [4]. Traditional regenerative medicine scaffolds are simply biocompatible and biodegradable, which do not fairly resemble the cell microenvironment. The extracellular matrix (ECM) in the human body is dynamic, hierarchical and tissue specific [5]. As a consequence, there is a need to develop bioactive scaffolds that could instruct basic cellular functions like adhesion, proliferation, and differentiation [4]. Such bioactive scaffolds can be constructed via designing and controlling the chemical, physical and mechanical properties of scaffolds. Physical and mechanical cues including surface topography, elasticity and structural features can regulate cell functions such as the above mentioned [6]. The chemistry of engineered scaffolds, which determine their surface chemistry, mechanical properties and biodegradable rate, are dependent on the class of materials [2]. Thus, an engineered scaffold with well-designed material chemistry, structural and mechanical properties is essential to steer the regenerative potential of stem cells [6, 7]. Among current fabrication methods, electrospinning remain a unique and effective technique to fabricate continuous nano or micro fibers. Due to its capacity to produce fibrous scaffolds resembling the features of ECM, electrospinning has been intensively investigated in regenerative medicine [8].

The aim of this thesis is to make a contribution in creating bioactive scaffolds for regenerative medicine and regenerative medicine through electrospinning-based techniques. To achieve this goal, here we mainly focus on designing new materials, tuning surface topography and developing new manufacturing techniques. Chapter 2 and chapter 3 show functional electrospun fibers were fabricated from new materials that are 
a new class of poly(butylene succinate)-based (PBS-based) polyesters and yttriumstabilized zirconia (YSZ) ceramics, respectively. In chapter 4, electrospun fibers encoded with different surface topographies were achieved by simply varying humidity during electrospinning. In chapter 5 , we have proposed a new approach to fabricate crimped electrospun fibers, which mimics the structural properties of collagenous fibers in different kinds of connective tissues, by using the principles of mechanical buckling through thermal shrinkable materials. Finally, in chapter 6 , we further developed a new solution electrospinning technique which allows depositing fibers in predetermined patters, in a controlled manner. This new direct writing electrospinning technology allows bridging the gap between additive manufacturing technologies and melt electrospinning and enabling to fabricate scaffolds with a more closely degree of biomimicry compared to native tissues. In chapter 7, a general discussion of the results presented in this thesis is hereafter elaborated and divided in the following sections.

\subsection{Fabrication of nanofibrous scaffold from PBS-based polyesters}

An essential requirement for ideal scaffolds in regenerative medicine is that they should have suitable mechanical properties and degradation rate to match tissue regeneration dynamics. Although commercial polyesters such as poly( $\varepsilon$-caprolactone) (PCL), poly(lactic acid) (PLA), poly(glycolic acid) (PGA) and copolymers (PLGA) have been broadly explored in regenerative medicine, they show limited flexibility in tuning mechanical properties and degradation rate. In addition, they lack of functions that can render chemical cues to steer cell differentiation. These disadvantages limited their application in regenerative medicine. Therefore, there was considerable demand for new polyesters. Commercial polyester named poly (butylene succinate) (PBS) could serve as a possible alternative to the above mentioned aliphatic polyesters due to its good thermal stability, proven biodegradability and biocompatibility, and low cost of raw materials [9, 10].

Chemical and physical modification of PBS may improve its performances with respect to the intended application. In chapter 2, functional electrospun scaffolds were fabricated from a class of multiblock PBS-based copolyesters containing either butylene thiodiglycolate (BTDG) or butylene diglycolate (BDG) sequences. These new polyesters displayed controllable wettability, hydrolysis and mechanical properties rate depending on the molecular architecture and on the kind of etheroatom introduced along the polymer backbone. The polymers containing BDG were more hydrophilic than those containing BTDG units attributed to the higher negative electric charge of oxygen atoms with respect 
to sulphur ones. The hydrolytic degradation rate of these scaffolds can be interrupted by crystallinity degree and surface hydrophilicity [11]. Copolymers containing BDG sequences degraded to a higher extent than those containing BTDG ones, due to the higher hydrophilicity of the former. The copolymer $P\left(B S_{11} B D G_{11}\right)$ degraded faster than its corresponding blend of PBS/PBDG, because of the lower crystallinity degree. $\mathrm{P}\left(\mathrm{BS}_{10} \mathrm{BTDG}_{10}\right)$ and its corresponding blend of PBS/PBTDG, they underwent a similar degradation profile because of their comparable crystallinity degree and hydrophilicity.

Previous studies have demonstrated that the chemical composition has a strong relationship with mechanical properties, degradation rate and hydrophilicity of the resulting scaffolds. For example, fibrin gels were reinforced with collagen and glycosaminoglycans to improve their mechanical strength [12]. The degradation rate of PLA can be increased by the incorporation of glycolide components in the polymer backbone chains through copolymerization [13]. The hydrophobicity of PLGA scaffolds can be physically modified by blending with the hydrophilic poly(ethylene glycol) [14]. The chemical composition of scaffolds is also known to be one of the key mediators of manipulating cell behavior such as cell attachment, proliferation, and differentiation [15, 16]. In the work by Chang et al., type II collagen and chondroitin sulfate were effectively grafted on PCL porous scaffolds to optimize the biocompatibility of the system [17]. They demonstrate that these modified scaffolds promote type II collagen and GAGs production from the seeded chondrocytes. Amino-functionalized coating surface are most favorable for osteogenic and adipogenic differentiation of human mesenchymal stem cells as compared to $\mathrm{OH}$-functionalized, $\mathrm{COOH}$ functionalized, and $\mathrm{CH}_{3}$-functionalized coating [15]. Here, we demonstrated that pure PBS polymers enhanced early osteogenic differentiation of hMSCs and the additional presence of ether units (BDG) resulted in enhanced mineralization.Vice versa, the presence of BTDG units in polymer chains enhanced the differentiation of human mesenchymal stromal cells (hMSCs) into chondrocytes while this chemistry inhibits their osteogenic differentiation.

Overall, these results highlight that scaffold chemistry is critical to its function and important to influence cell activity. Thus, chemical properties of scaffolds should be taken into stronger consideration when designing scaffolds for regenerative medicine application. 


\subsection{Fabrication of bioactive nanofibrous scaffolds via tuning structure and texture}

Besides polymers, ceramics are also an important material candidate for regenerative medicine. Although ceramics including hydroxyapatite (HA) and tricalcium phosphate (TCP) have been widely used as scaffolds for skeletal tissue regeneration, the principle drawback of these materials is their brittleness. Among the ceramics so far studied, zirconia is widely used for orthopedic and dental implantation due to its excellent biological and mechanical properties [18]. However, zirconia is a bio-inert material, which restricts its application in regenerative medicine [19]. To overcome the aforementioned problems, in chapter 3, novel paper-like flexible nanofibrous YSZ scaffolds were prepared via electrospinning a mixture of YSZ precursors and polyvinylpyrrolidone (PVP), followed by annealing the green fiber in either a conventional oven or microwave. Electrospun silica-based mats were flexible than crystalline materials owing to the amorphous nature of the material [20]. However, these new YSZ nanofiber mats, which were crystallized, showed remarkable flexibility compared to traditional ceramics. The mats could withstand a total deflection of $180^{\circ}$ bending and cycled at least 1000 times without mechanical failure. This phenomenon may be attributed to the long length of the fabricated nanofibers leading to physical entanglement. Nevertheless, individual nanofibers offer stiffness values close to bulk zirconia, 5 orders of magnitude stiffer than the mat. Live and dead assay showed high cell viability on the developed nanofibrillar scaffolds. Gene expression and ALP activity of seeded hMSCs was promoted by the nanofibrous structure of zirconia scaffolds compared to the bulk ceramic. Interestingly, significant higher amount of calcium content was observed on the scaffolds, in contrast to the well-known bioinert behavior of bulk zirconia. These results suggested that electrospun fiber matrices provide a more suitable environment for osteogenic differentiation of hMSCs. This difference could be ascribed to their structural features, since their scaffold chemistry and culture condition were the same. The morphology and fiber dimension of electrospun fibers are comparable with those observed in the ECM of human. Thus, electrospun nanofibrous scaffolds provide improved environments for cell responds including cell attachment, proliferation, and differentiation [21]. Hae-Won Kim et al. demonstrated bone marrow derived stem cells had enhanced osteogenic potential on glass nanofibers than glass disks due to their nanofibrous structure [22].

Apart from structural features elaborated above, other physical cues can also be used as potent regulators of cell behavior such as surface topography and stiffness of scaffolds, mechanical force (like shear stress imposed by culture media) and external force (like 
light signal) [7,23]. Recently studies have shown that the design of surface topography of materials can be used for controlling stem cells differentiation [24, 25]. Increasing the surface roughness and surface porosity of materials causes an improvement in cellular attachment and differentiation [26]. In Chapter 3, we demonstrated that the modulation in surface roughness of zirconia ceramic fibers could be achieved by applying different sintering mechanisms. Nanofibers annealed in a conventional oven showed a surface roughness of $0.7 \pm 0.2 \mathrm{~nm}$ while nanofibers annealed in a microwave oven had a roughness of $0.4 \pm 0.1 \mathrm{~nm}$. A step-bunching effect (i.e., aggregation of the ceramic steps), sudden shrinkage and densification, and/or improved oxygen transport can be used to explain the formation of smooth surface when annealed in MW [29-31].However, modulating the heating rate did not show obvious influence on surface morphology among samples that were annealed by the same heating mechanism. It was worth to notice that the grain sides of samples depended on heating rates of annealing and heating mechanism. Using a lower heating rate would cause larger grain sizes due to the longer effective sintering times. Conversely, the samples annealed in a MW showed a smaller grain size than the one annealed in a $\mathrm{CO}$ using the same heating rate. A similar result was reported by Xie et al. who demonstrated smaller and more uniform grain sizes for YSZ when annealed in a MW [32]. It has been demonstrated that surface topographical cues influence cellular activity such as adhesion [27, 33], migration [34], proliferation [27], and differentiation [35, 36]. The results of mineralization assay revealed that the rougher scaffolds annealed in CO promoted higher calcium content compared to the MW annealed smooth scaffolds $(0.4 \pm 0.1 \mathrm{~nm})$. This finding was consistent with the previous work where a rougher titanium surface promoted matrix mineralization of osteoblasts compare to smooth titanium surface.

As a logical consequence of the findings of chapter 3 , the influence of surface roughness of electrospun fibers on cell activity was further investigated on polymeric scaffolds (chapter 4). By varying the relative humidity during electrospinning, it was possible to make fibers with different surface roughnesses, yet keeping constant other scaffolds properties including fiber dimension, scaffold pores and porosity, and scaffold chemistry. Another advantage of this approach lies in a homogeneous modification, which is applied to every single fiber of the scaffolds rather than happening on the surface area of scaffolds. In chapter 4 , electrospun fibers having varied surface roughness were fabricated from copolymer PEOT/PBT under different humidity condition. The surface roughness (Ra) of obtained fibers increased from $14.3 \mathrm{~nm}$ to $71.0 \mathrm{~nm}$ when the humidity increased from 
$20 \%$ to $70 \%$ during electrospinning. These results are in line with the work reported by Casper et al. where increasing humidity caused an increase of pores on the surface of polystyrene fibers [37]. Adsorption is a surface phenomenon. The amount of adsorbed protein on a surface is dependent on the surface roughness and chemistry [38]. The fibers fabricated from $70 \%$ relative humidity had a higher protein adsorption compared to the one obtained from $20 \%$ relative humidity due to the higher surface roughnesses of the former one. In addition, surface energy is also influenced by surface topography. Fibers having a surface roughness of $71.0 \mathrm{~nm}$ showed a different surface wettability compared to the one with a surface roughness of $14.3 \mathrm{~nm}$. Similar results were reported by Chuah et al. who demonstrated that increasing the surface roughness caused variation in surface tension

hMSCs were cultured for 21 days on scaffolds with different surface roughnesses. We found that the influence of surface roughnesses on hMSCs osteogenesis was medium dependent. The control medium (basic medium) showed much more pronounced effect compared to the differentiation medium (osteogenic medium). In basic medium, some specific osteogenic genes (OPN and BMP2) displayed higher expression on a higher surface roughness $(\mathrm{Ra}=71.0 \mathrm{~nm})$, whereas other genes, including $\mathrm{BSP}, \mathrm{COL} 1 \mathrm{~A} 1$ and $\mathrm{OCN}$, showed higher expression on a lower surface roughness ( $\mathrm{Ra}=14.3 \mathrm{~nm})$. In osteogenic medium, the result was not conclusive. In addition, a higher surface roughness $(\mathrm{Ra}=71.0 \mathrm{~nm})$ had higher ALP activity than a lower surface roughness ( $R a=14.3 \mathrm{~nm})$. A recent study demonstrated that a surface composed of nano and sub-micron mixed features promote better osteogenic differentiation of hMSCs compared to the surface only containing singular features (nano or sub-micron scale) [39]. This can be used to explain the finding that both lower and higher roughnesses here investigated supported osteogenic differentiation of hMSCs. In the case of hMSCs chondrogenesis, a lower surface roughness $(\mathrm{Ra}=14.3 \mathrm{~nm})$ showed to better support the chondrogenic differentiation of hMSCs at day 7 compared to higher surface roughness $(\mathrm{Ra}=71.02 \mathrm{~nm})$ in differentiation medium. At a protein level, no significant difference in GAG content was found among the rougnesses tested in the present study.

\subsection{Developing new manufacturing techniques}

One of the principles in regenerative medicine is that scaffolds should resemble the ECM of the tissues to be replaced. There are numerous types of ECM in human tissues and their functions are tissue-specific. For example, collagen fibers, one of the main components of ECM, present crimped structure in several human tissues, such as tendon, ligament, 
muscle, and dermis. In these tissues, the wavy collagen structure gives its tissue structural and mechanical properties. For instance, the wavy collagen fibers in tendon, which are highly resistant to large stretching, is associated with the high tensile strength of tendons. Despite electrospinning has been extensively used in regenerative medicine, little progress has been made for fabricating crimped fibers mimicking the fibril pattern of natural tissues. In chapter 5, we developed a new technique for generating wavy patterns in electrospun fibers. This new technique comprise three steps: first, a construct was achieved by depositing electrospun fibers on a thermally shrinkable material; second, by heating the construct above the glass temperature of the shrinkable material results in shrinkage, thereby buckling the adhered fibers; third, by cooling down the construct at room temperature, the curl pattern is retained. In general, the developed technique is a simple and effective approach to fabricate crimped fibers and showed following advantages compared to previous studies [40-42]: i) the deposited fibers adhere to thermally shrinking film without extra bonding process; ii) the buckled fiber patterns are retained by cooling down at room temperature without extra fixating agents or methods; iii) a wide range of crimped patterns can be achieved by tuning the processing conditions; iv) the capability to create multiscale crimped patterns spanning from fibers to fiber meshes by controlling the thickness of fiber layers, the depositing time of fibers and the shape or geometry of the thermo-shrinkable materials.

Cellular migration is one of the major concerns for electrospun scaffolds in terms of their application in regenerative medicine. hMSCs present a more even distribution and cellular infiltration on wavy scaffolds compared to non-wavy scaffolds. This could be attributed to the wavy patterns which increase of free volume inside the scaffold. The wavy scaffolds have a pattern mimicking the natural configuration of connective tissues, such as tendons and ligaments. It was demonstrated that such topographical pattern supported the expression of TGF- $\beta$ which is a key signal pathway related to connective tissue development. Some further investigations are still necessary to evaluate if the same trend could be observed on more specific cell populations than the mink lung epithelial cell line used on the scaffolds in this study. The present technique in chapter 5 is simple and universal, which can also be translated to other polymers and thermo-shrinkable materials. We have shown successful translation to polymers including PVA, PAN and PS through using PLA shrinkable films. In addition, PEOT/PBT and PAN fibers having wavy patterns were also obtained using PS biaxial shrinkable films. Thus, the technique shown in chapter 5 could serve as a technical platform to fabricate functional scaffolds that could be 
beneficial for mimicking a variety of tissues organization, such as tendons, ligaments, trachea, muscles, skin, and the connective tissues of the eyes.

Electrospinning, which was first studied in the 1930s, remains one of the most effective approaches for generating nanofibers [43]. One of the main issues for traditional electrospining lies in the difficulty to control the morphology and geometry of the electrospun meshes, resulting from the rapidly whipping electrospinning jet [44, 45]. Despite the fact that attempts to control the fiber deposition in an accurate manner using melting electrospinning writing (MEW) have been reported, fibers produced by melt electrospinning usually have larger dimensions compared to conventional solution electrospinning. Another notable drawback of melt electrospinning is the use of elevated temperature, which may cause the degradation of thermo-sensitive polymers. Furthermore, many natural polymers including collagen cannot work with melt electrospinning [46]. In addition to MEW technique, near-field electrospinning (NFESP) have been developed as a process capable of depositing nanofibers in a controllable and continuous manner. The NFESP is characterized by using a shorter spinneret-to-collector distance together with very low voltages. However, this technique has difficulty in fabricating large scale scaffolds as it relies on a solution-dipped probe for jet feeding [47].To solve the above mentioned problems, one possible solution is to develop a new technique which allows solution electrospining to deposit fibers in a controlled manner. Recently, solution electrospining have been reported to fabricate patterned nanofibrous mats [45, 48]. However, in these cases, the used apparatus was conventional electrospining in combination of extra components including a side-wall, scanning functions and sharp-pin electrode, which were required to improve the focusing $[45,48]$. In contrast to the setup they used, the solution electrospining apparatus utilized in chapter 6 is much simpler. It was only composed of a conventional electrospining system able to move in an $x-y-z$ programmable manner. Interestingly, three different regimes could be obtained: the fiber deposition into a bundle of random fibers, a bundle with aligned fibers at its core, and single fiber deposition. The transition between these regimes was dependent on the applied working distance and the resulting electrical field strength. The transition between regimes of fiber deposition into a bundle of random fibers and a single unit has been demonstrated in previous studies [45, 49]. However, to the best of our knowledge, the deposition of fibers into a bundle with aligned at core through a direct writing electrospinning process have not been reported yet.

The ECM in the human body is complex and tissue specific with an often hierarchically organized structure $[4,46]$. For example, articular cartilage is a highly organized tissue with 
different functional zones [50-52]. Articular cartilage has limited capacity to self-repair after being damaged [53-55]. For this reason, the development of solutions that aid in the regeneration process of damaged articular cartilage remains an important issue in the regenerative medicine field. In this respect, we attempted to design a new fictional scaffold for cartilage repair. With the new technique, we fabricated lattice patterned mats that mimic the zonal organization of collagen fibers in articular cartilage. Four lattice patterned mats were further stack together to form a final functional construct. Compared to the typical electrospun mesh, the direct writing electrospinning (DW-ESP) scaffold was able to direct tissue and ECM fibril organization in a zone dependent manner. Additionally, some chondrogenic gene markers, including Sox9 and aggrecan (ACAN) were significantly upregulated in this structure. The new technique provides a new approach for patterning structures mimicking the configuration of virtually any human tissues. However, some major issues relating to this technique still need to be addressed in the future: for example, to expand the amount of biomaterials that can be used with DW-ESP and to overcome the problem of charge repulsion to obtain thicker constructs in a more reliable manner.

\subsection{Future Perspective}

Nanofibrous scaffolds should be designed more and more as bioactive systems rather than just passive cell carriers. There are some important factors, such as mechanical properties, degradation rate, and geometrical structure and texture that need to be addressed in the "design stage" for tailoring nanofibrous scaffolds. All of these factors play an important role in governing cellular responses and host integration. Future research on bioactive artificial nanofbrous scaffolds may focus on the following aspects: The first strategy is to dig deeper in the basic mechanisms of tissue formation in biological systems. In the human body, there are multiple types of tissues of which structures are complex and tissue specific. The mechanisms underlying the formation, repair and maintenance of human tissue are not well understood. Without this fundamental knowledge that is crucial to custom-design functional scaffolds, regenerative medicine would practice in blindness. Secondly, developing and improving new techniques for manufacturing nanofibrous scaffolds is always necessary. Currently, phase separation, electrospinning, and self-assembling are the most popular approaches for fabrication of nanofibrous scaffolds. However, all these methods do not fully meet the increasingly demand for designing smart and bioactive scaffolds having tailored functions. In future studies, we need to fully explore the current techniques of fabricating 
nanofibrous scaffolds. For example, we should understand how to improve direct writing electrospinning, which would allow depositing fibers forming a thick cm-scaled 3D dimension construct. Another approach is to integrate electrospinning with other techniques, which open up much possibility to manufacture artificial functional scaffolds. Electrospining combined with other surface modification methods, such as plasma, lithography, ultrasonic spray coating and chemical grafting, could be used to generate bioactive topographic cues for the desired scaffold's function. For instance, through using electrospinning and nanoimprinting techniques, nanofibrous scaffolds could be decorated with nanotopographic patterns, such as ridges and grooves, to better match the nanostructure of ECM [56]. Nanofibrous scaffolds having a hierarchical structure could be achieved by integrating self-assembling or phase separation processes with 3D printing techniques. Nanofibrous hydrogels, which were self-assembled from amphiphilic peptides, were used as bioink for printing 3D scaffolds with a predesigned pattern [57]. Thirdly, the release of growth factor from electrospun scaffolds could be envisioned. Growth factors which can induce angiogenesis are often required to promote tissue formation [58]. However, direct injection of soluble growth factors into the defect site will compromise the biological effect of growth factors due to a rapidly diffusion away from the injection site. Thus, scaffolds are used as drug release systems to maintain sustained release of grow factors. An ideal engineered release system for growth factor should resemble their natural release system in the human body. Lastly, developing new materials is also essential for designing bioactive scaffolds. The function and properties of scaffold are mainly determined by its materials chemistry. It would be attractive to design new materials which could induce stem cell differentiation, via high throughput screening of chemistry. It would be interesting to develop new materials that would not evoke immune response and inflammation in vivo.

Although much joint effort by scientists from multiple disciplines is still needed for the development of nanofibrous scaffolds in different regenerative medicine applications, it can be foreseen that nanofibrous scaffolds could approach "off-the-shelf" surgically implantable constructs in the near future. 


\section{Reference}

[1] R.H. Harrison, J.-P. St-Pierre, M.M. Stevens, Tissue engineering and regenerative medicine: a year in review, Tissue Engineering Part B: Reviews 20(1) (2014) 1-16.

[2] W.J. Li, C.T. Laurencin, E.J. Caterson, R.S. Tuan, F.K. Ko, Electrospun nanofibrous structure: a novel scaffold for tissue engineering, J. Biomed. Mater. Res. 60(4) (2002) 613-621.

[3] R.P. Pirraco, R.L. Reis, Tissue Engineering: New Tools for Old Problems, Stem cell reviews 11(3) (2015) 373.

[4] T. Dvir, B.P. Timko, D.S. Kohane, R. Langer, Nanotechnological strategies for engineering complex tissues, Nature nanotechnology 6(1) (2011) 13-22.

[5] K.Y. Tsang, M.C. Cheung, D. Chan, K.S. Cheah, The developmental roles of the extracellular matrix: beyond structure to regulation, Cell Tissue Res. 339(1) (2010) 93-110.

[6] H.-J. Park, S.J. Yu, K. Yang, Y. Jin, A.-N. Cho, J. Kim, B. Lee, H.S. Yang, S.G. Im, S.-W. Cho, Paperbased bioactive scaffolds for stem cell-mediated bone tissue engineering, Biomaterials 35(37) (2014) 98119823.

[7] A. Higuchi, Q.-D. Ling, Y. Chang, S.-T. Hsu, A. Umezawa, Physical cues of biomaterials guide stem cell differentiation fate, Chemical reviews 113(5) (2013) 3297-3328.

[8] T. Jiang, E.J. Carbone, K.W.-H. Lo, C.T. Laurencin, Electrospinning of polymer nanofibers for tissue regeneration, Progress in Polymer Science 46 (2015) 1-24.

[9] M. Gigli, M. Fabbri, N. Lotti, R. Gamberini, B. Rimini, A. Munari, Poly (butylene succinate)-based polyesters for biomedical applications: A review, European Polymer Journal 75 (2016) 431-460.

[10] I. Bechthold, K. Bretz, S. Kabasci, R. Kopitzky, A. Springer, Succinic acid: a new platform chemical for biobased polymers from renewable resources, Chemical engineering \& technology 31(5) (2008) 647-654.

[11] M. Gigli, A. Negroni, M. Soccio, G. Zanaroli, N. Lotti, F. Fava, A. Munari, Influence of chemical and architectural modifications on the enzymatic hydrolysis of poly (butylene succinate), Green Chemistry 14(10) (2012) 2885-2893.

[12] C.M. Brougham, T.J. Levingstone, S. Jockenhoevel, T.C. Flanagan, F.J. O’Brien, Incorporation of fibrin into a collagen-glycosaminoglycan matrix results in a scaffold with improved mechanical properties and enhanced capacity to resist cell-mediated contraction, Acta biomaterialia 26 (2015) 205-214.

[13] K. Kim, M. Yu, X. Zong, J. Chiu, D. Fang, Y.-S. Seo, B.S. Hsiao, B. Chu, M. Hadjiargyrou, Control of degradation rate and hydrophilicity in electrospun non-woven poly ( $\mathrm{D}$, L-lactide) nanofiber scaffolds for biomedical applications, Biomaterials 24(27) (2003) 4977-4985.

[14] V.P. Shastri, I. Martin, R. Langer, Macroporous polymer foams by hydrocarbon templating, Proceedings of the National Academy of Sciences 97(5) (2000) 1970-1975.

[15] J.E. Phillips, T.A. Petrie, F.P. Creighton, A.J. García, Human mesenchymal stem cell differentiation on self-assembled monolayers presenting different surface chemistries, Acta biomaterialia 6(1) (2010) 12-20.

[16] B.D. Boyan, T.W. Hummert, D.D. Dean, Z. Schwartz, Role of material surfaces in regulating bone and cartilage cell response, Biomaterials 17(2) (1996) 137-146.

[17] K.Y. Chang, L.H. Hung, I. Chu, C.S. Ko, Y.D. Lee, The application of type II collagen and chondroitin sulfate grafted PCL porous scaffold in cartilage tissue engineering, Journal of Biomedical Materials Research Part A 92(2) (2010) 712-723. 
[18] P.F. Manicone, P.R. Iommetti, L. Raffaelli, An overview of zirconia ceramics: basic properties and clinical applications, Journal of dentistry 35(11) (2007) 819-826.

[19] Y.-T. Liu, T.-M. Lee, T.-S. Lui, Enhanced osteoblastic cell response on zirconia by bio-inspired surface modification, Colloids and Surfaces B: Biointerfaces 106 (2013) 37-45.

[20] M. Guo, B. Ding, X. Li, X. Wang, J. Yu, M. Wang, Amphiphobic nanofibrous silica mats with flexible and high-heat-resistant properties, The Journal of Physical Chemistry C 114(2) (2009) 916-921.

[21] X. Li, L. Wang, Y. Fan, Q. Feng, F.Z. Cui, F. Watari, Nanostructured scaffolds for bone tissue engineering, Journal of Biomedical Materials Research Part A 101(8) (2013) 2424-2435.

[22] H.W. Kim, H.E. Kim, J.C. Knowles, Production and Potential of Bioactive Glass Nanofibers as a NextGeneration Biomaterial, Advanced Functional Materials 16(12) (2006) 1529-1535.

[23] A. Higuchi, Q.-D. Ling, S.S. Kumar, Y. Chang, A.A. Alarfaj, M.A. Munusamy, K. Murugan, S.-T. Hsu, A. Umezawa, Physical cues of cell culture materials lead the direction of differentiation lineages of pluripotent stem cells, Journal of Materials Chemistry B 3(41) (2015) 8032-8058.

[24] T.A. Mitsiadis, A. Woloszyk, L. Jiménez-Rojo, Nanodentistry: combining nanostructured materials and stem cells for dental tissue regeneration, Nanomedicine 7(11) (2012) 1743-1753.

[25] M. Vallet-Regí, A.J. Salinas, D. Arcos, Tailoring the Structure of Bioactive Glasses: From the Nanoscale to Macroporous Scaffolds, International Journal of Applied Glass Science (2016).

[26] D.O. Costa, B.A. Allo, R. Klassen, J.L. Hutter, S.J. Dixon, A.S. Rizkalla, Control of surface topography in biomimetic calcium phosphate coatings, Langmuir 28(8) (2012) 3871-3880.

[27] D.D. Deligianni, N.D. Katsala, P.G. Koutsoukos, Y.F. Missirlis, Effect of surface roughness of hydroxyapatite on human bone marrow cell adhesion, proliferation, differentiation and detachment strength, Biomaterials 22(1) (2000) 87-96.

[28] J.Y. Lim, H.J. Donahue, Cell sensing and response to micro-and nanostructured surfaces produced by chemical and topographic patterning, Tissue engineering 13(8) (2007) 1879-1891.

[29] J. Wang, J. Binner, B. Vaidhyanathan, N. Joomun, J. Kilner, G. Dimitrakis, T.E. Cross, Evidence for the microwave effect during hybrid sintering, Journal of the American Ceramic Society 89(6) (2006) 1977-1984.

[30] S.G. Sundaresan, M.V. Rao, Y.-I. Tian, M.C. Ridgway, J.A. Schreifels, J.J. Kopanski, Ultrahightemperature microwave annealing of $\mathrm{Al+-}$ and P+-implanted 4H-SiC, Journal of Applied Physics 101(7) (2007) - .

[31] A. Goldstein, N. Travitzky, A. Singurindy, M. Kravchik, Direct microwave sintering of yttria-stabilized zirconia at 2.45\&\#xa0;GHz, Journal of the European Ceramic Society 19(12) (1999) 2067-2072.

[32] Z. Xie, J. Yang, X. Huang, Y. Huang, Microwave processing and properties of ceramics with different dielectric loss, Journal of the European Ceramic Society 19(3) (1999) 381-387.

[33] F. Zamani, M. Amani-Tehran, M. Latifi, M.A. Shokrgozar, The influence of surface nanoroughness of electrospun PLGA nanofibrous scaffold on nerve cell adhesion and proliferation, Journal of Materials Science: Materials in Medicine 24(6) (2013) 1551-1560.

[34] J. Song, J.H. Shawky, Y. Kim, M. Hazar, P.R. LeDuc, M. Sitti, L.A. Davidson, Controlled surface topography regulates collective 3D migration by epithelial-mesenchymal composite embryonic tissues, Biomaterials 58 (2015) 1-9. 
[35] M.J. Dalby, N. Gadegaard, R. Tare, A. Andar, M.O. Riehle, P. Herzyk, C.D. Wilkinson, R.O. Oreffo, The control of human mesenchymal cell differentiation using nanoscale symmetry and disorder, Nature materials 6(12) (2007) 997-1003.

[36] A.B. Faia-Torres, S. Guimond-Lischer, M. Rottmar, M. Charnley, T. Goren, K. Maniura-Weber, N.D. Spencer, R.L. Reis, M. Textor, N.M. Neves, Differential regulation of osteogenic differentiation of stem cells on surface roughness gradients, Biomaterials 35(33) (2014) 9023-9032.

[37] R. Chaim, M. Hefetz, Effect of grain size on elastic modulus and hardness of nanocrystalline ZrO2-3 wt\% Y2O3 ceramic, Journal of materials science 39(9) (2004) 3057-3061.

[38] A. Nandakumar, Z.T. Birgani, D. Santos, A. Mentink, N. Auffermann, K. van der Werf, M. Bennink, L. Moroni, C. van Blitterswijk, P. Habibovic, Surface modification of electrospun fibre meshes by oxygen plasma for bone regeneration, Biofabrication 5(1) (2013) 015006.

[39] D. Khang, J. Choi, Y.-M. Im, Y.-J. Kim, J.-H. Jang, S.S. Kang, T.-H. Nam, J. Song, J.-W. Park, Role of subnano-, nano-and submicron-surface features on osteoblast differentiation of bone marrow mesenchymal stem cells, Biomaterials 33(26) (2012) 5997-6007.

[40] D.C. Surrao, J.W. Hayami, S.D. Waldman, B.G. Amsden, Self-crimping, biodegradable, electrospun polymer microfibers, Biomacromolecules 11(12) (2010) 3624-3629.

[41] J.M. Caves, V.A. Kumar, W. Xu, N. Naik, M.G. Allen, E.L. Chaikof, Microcrimped Collagen FiberElastin Composites, Advanced Materials 22(18) (2010) 2041-2044.

[42] D.C. Surrao, J.C. Fan, S.D. Waldman, B.G. Amsden, A crimp-like microarchitecture improves tissue production in fibrous ligament scaffolds in response to mechanical stimuli, Acta biomaterialia 8(10) (2012) 3704-3713.

[43] B. Sun, Y. Long, H. Zhang, M. Li, J. Duvail, X. Jiang, H. Yin, Advances in three-dimensional nanofibrous macrostructures via electrospinning, Progress in Polymer Science 39(5) (2014) 862-890.

[44] M.M. Hohman, M. Shin, G. Rutledge, M.P. Brenner, Electrospinning and electrically forced jets. I. Stability theory, Physics of Fluids (1994-present) 13(8) (2001) 2201-2220.

[45] J. Lee, S.Y. Lee, J. Jang, Y.H. Jeong, D.-W. Cho, Fabrication of patterned nanofibrous mats using direct-write electrospinning, Langmuir 28(18) (2012) 7267-7275.

[46] S. Khorshidi, A. Solouk, H. Mirzadeh, S. Mazinani, J.M. Lagaron, S. Sharifi, S. Ramakrishna, A review of key challenges of electrospun scaffolds for tissue-engineering applications, Journal of tissue engineering and regenerative medicine (2015).

[47] F. Ruggieri, D. Di Camillo, L. Lozzi, S. Santucci, A. De Marcellis, G. Ferri, L. Giancaterini, C. Cantalini, Preparation of nitrogen doped TiO 2 nanofibers by near field electrospinning (NFES) technique for NO 2 sensing, Sensors and Actuators B: Chemical 179 (2013) 107-113.

[48] J. Lee, J. Jang, H. Oh, Y.H. Jeong, D.-W. Cho, Fabrication of a three-dimensional nanofibrous scaffold with lattice pores using direct-write electrospinning, Materials Letters 93 (2013) 397-400.

[49] T.D. Brown, P.D. Dalton, D.W. Hutmacher, Direct writing by way of melt electrospinning, Advanced Materials 23(47) (2011) 5651-5657.

[50] J. Steele, S. McCullen, A. Callanan, H. Autefage, M. Accardi, D. Dini, M. Stevens, Combinatorial scaffold morphologies for zonal articular cartilage engineering, Acta biomaterialia 10(5) (2014) 2065-2075.

[51] R. Minns, F. Steven, The collagen fibril organization in human articular cartilage, Journal of anatomy 123(Pt 2) (1977) 437. 
[52] N.S. Landínez-Parra, D.A. Garzón-Alvarado, J.C. Vanegas-Acosta, Mechanical Behavior of Articular Cartilage, INTECH Open Access Publisher2012.

[53] H. Xu, S. Cai, L. Xu, Y. Yang, Water-stable three-dimensional ultrafine fibrous scaffolds from keratin for cartilage tissue engineering, Langmuir 30(28) (2014) 8461-8470.

[54] J.X. Xue, Y.Y. Gong, G.D. Zhou, W. Liu, Y. Cao, W.J. Zhang, Chondrogenic differentiation of bone marrow-derived mesenchymal stem cells induced by acellular cartilage sheets, Biomaterials 33(24) (2012) $5832-5840$.

[55] J.M. Coburn, M. Gibson, S. Monagle, Z. Patterson, J.H. Elisseeff, Bioinspired nanofibers support chondrogenesis for articular cartilage repair, Proceedings of the National Academy of Sciences 109(25) (2012) 10012-10017.

[56] A. Nandakumar, R. Truckenmüller, M. Ahmed, F. Damanik, D.R. Santos, N. Auffermann, J. de Boer, P. Habibovic, C. van Blitterswijk, L. Moroni, A fast process for imprinting micro and nano patterns on electrospun fiber meshes at physiological temperatures, Small 9(20) (2013) 3405-3409.

[57] Y. Loo, A. Lakshmanan, M. Ni, L.L. Toh, S. Wang, C.A. Hauser, Peptide bioink: self-assembling nanofibrous scaffolds for three-dimensional organotypic cultures, Nano letters 15(10) (2015) 6919-6925.

[58] Y. Tabata, Tissue regeneration based on growth factor release, Tissue Engineering 9(4, Supplement 1) (2003) 5-15. 


\section{Chapter 8}

\section{Valorisation}

Scientific knowledge does not, by itself, result in widespread implementation and positive social impacts. The research from the laboratory must be translated in to practical use. This process, which is about creating impact through transfer scientific knowledge, is also called 'valorisation of knowledge' or 'technology transfer'. Valorisation has been defined by Netherlands Proteomics Centre as "The process of value-creation out of knowledge, by making this knowledge suitable and available for economic or societal utilisation and to translate this into high-potential products, services, processes and industrial activity"[1]. The research in this dissertation is on fabrication of bioactive scaffolds for regenerative medicine. Some of the findings show high potential for valorization, especially, the one in chapter 5 where a novel technique is developed to fabricate nanofibrous scaffolds with tailored patterns. In the following sections, we will focus on elaborating the possible valorization options for chapter 5. First, I will start the discussion from a social and clinical relevance, and subsequently I will discuss about the novelty concept and potential application.

\subsection{Social and clinical relevance}

Tendon is a tough bundle of fibrous connective tissue that transfer forces between bone and muscle [2, 3]. Like low vascularized tissue of articular cartilages, tendons are characterized with low cell density, low oxygen and nutrient requirement, and poor healing capacity [2, 4]. The injury of tendon is common. For example, the yearly incidence for Achilles tendon is roughly 18 per 100,000 in the world and this number is thought to increase rapidly due to the growth of sports participation and ageing of the world population [5]. Given their poor self-regenerative capacity, surgical management is the only recourse for large tendon injury[4]. Unfortunately, current surgical reparative approaches which are often associated with auto- or allografts replacement, could not fully restore their functional and structural properties. Recently, tissue engineering has been established as a promising strategy for tendon regeneration. Scaffolds are considered as one of the most important principle elements of tissue engineering. 
Currently, electrospinning has been extensively used to fabricate scaffolds for tendon repair due to its capacity to continuously generate nanofibers. However, the traditional electrospinning technique has difficulty in fabricating scaffolds with buckled patterns, which are of important topographical cues in the development of connective tissue, including tendon. Thus, we invented a new technique to overcome this issue.

\subsection{Novelty of the concept}

Chapter 5 describes a new method of making wavy fibers, comprising: (a) depositing electrospun fibers on a surface of a thermally shrinkable material, which material is shrinkable in at least one direction, wherein the deposited fiber at least partially adheres to the shrinkable material; (b) shrinking the shrinkable material in at least one direction, thereby buckling the fiber. The technique presented here is simple and low cost compared to prior arts [6,7]. Firstly, the deposited fibers will attach to the thermal shrinkable materials automatically without extra bonding process. Secondly, extra fixating agents or methods are not required for retaining the wavy pattern. To the best of our knowledge, this is the first time we present a technique that is capable to generate buckled patterns on individual fibers, shifting to buckling the whole fibrous mesh through configuring fiber depositing time. Another notable advantage of the technique described in chapter 5 is that wavy patterns could be regulated in a wide range of options, such as verifying the orientation of depositing fibers, using a second layer of a water-soluble polymer to change the amplitude and frequency of the wavy patterns, and varying the geometry shape and kind of shrinkable materials. The pilot cell biology study demonstrated that the obtained wavy scaffolds promoted cellular penetration, which is one of the major concerns in terms of electrospun scaffolds for tissue engineering applications. In addition, pilot studies indicated that the obtained wavy scaffolds promoted TGF- $\beta$ signaling expression that is a key signaling pathway involved in the development of several connective tissues including tendon.

\subsection{Potential application}

The results described in this dissertation may open doors to the development of fabricating functional scaffolds for tissue regeneration, especially where wavy topographical cues were needed. The successful translation of this technique described here toward society, firstly and most importantly, may benefit a great variety of patients who need to recover from tendon injury. The following approaches could be adopted to put this novel technique in the market. Firstly, it is worth filing a patent application for this 
technique; secondly, a startup company could be formed to aim at producing scaffolds for tissue engineering. However, before producing the final products, further studies on growing more specific cells (such as tendon cell lines) on the obtained wavy scaffolds should be performed, followed by validation in a functional animal model. Thirdly, this technique could be sold to the market directly, for example, to those companies that are already active with electrospinning products. 


\section{Reference}

[1] Netherlands Proteomic Centre. What is valorisation? .

$<$ http://www.netherlandsproteomicscentre.nl/npc/valorisation/what-is-valorisation>, (cited on 8th March.2017).

[2] C.K. Kuo, J.E. Marturano, R.S. Tuan, Novel strategies in tendon and ligament tissue engineering: advanced biomaterials and regeneration motifs, BMC Sports Science, Medicine and Rehabilitation 2(1) (2010) 20.

[3] G. Yang, B.B. Rothrauff, R.S. Tuan, Tendon and ligament regeneration and repair: clinical relevance and developmental paradigm, Birth Defects Research Part C: Embryo Today: Reviews 99(3) (2013) 203222.

[4] A. Moshiri, A. Oryan, Tendon and ligament tissue engineering, healing and regenerative medicine, J Sports Med Doping Stud 3(126) (2013) 2161-0673.1000126.

[5] J.T. Shearn, K.R. Kinneberg, N.A. Dyment, M.T. Galloway, K. Kenter, C. Wylie, D.L. Butler, Tendon tissue engineering: progress, challenges, and translation to the clinic, Journal of musculoskeletal \& neuronal interactions 11(2) (2011) 163.

[6] H. Schirmer, Fabric formed by heat sealing, shrinking and foaming backing, Google Patents, 1973.

[7] J.M. Caves, V.A. Kumar, W. Xu, N. Naik, M.G. Allen, E.L. Chaikof, Microcrimped collagen fiber-elastin composites, Advanced Materials 22(18) (2010) 2041-2044. 


\section{Summary}

In this thesis, a number of novel strategies to design and fabricate instructive scaffolds for the regeneration of skeletal tissues are presented. Chapter 1 describes recent advanced in technologies for manufacturing nanofibrous scaffolds with a structure resembling the native ECM. Strategies for improving cell-scaffold interaction were also illustrated before discussing nanofibrous scaffolds for tissue engineering and regenerative medicine applications. From the review of literature proposed in chapter 1, it appears that traditional nanofibrous scaffolds do not display instructive properties, despite their physical resemblance to the native ECM. Thus, there is a great need for fabricating bioactive fibrillar scaffolds, which are capable to instruct cellular functions like adhesion, proliferation, and differentiation. Such bioactive scaffolds could be constructed via tailoring their physical properties (e.g. surface topography, mechanical properties, and structural features), and chemistry.

Chapter 2 shows functional electrospun fibers were fabricated from a new class of poly(butylene succinate)-based (PBS-based) polyesters containing either butylene thiodiglycolate (BTDG) or butylene diglycolate (BDG) sequences. Controllable properties including mechanical strength, wettability, and hydrolysis, could be achieved for these scaffolds through manipulating the molecular architecture and introducing an etheroatom along the polyesters backbone. Polyesters containing BDG sequences were more hydrophilic compared to those containing BTDG units. The higher hydrophilicity of polyesters led to a higher degradation rate. The polymer chemistry of the scaffolds also showed to have an important effect on cell-scaffold interactions. Here, the pure PBS scaffolds were beneficial to stimulate early hMSCs osteogenic differentiation. With the introduction of ether units (BDG) along its polymer backbone, the scaffolds showed enhanced mineralization properties. Having the presence of BTDG functional sequences in the polymer chains, scaffolds supported hMSCs chondrogenisis, while inhibiting their osteogenic differentiation. Tailoring the chemistry composition of nanofibrous scaffold could, therefore, serve as an appealing strategy for deigning bioactive scaffolds in the regenerative medicine field.

Apart from the chemistry point of view, the structure and texture are also essential for functional scaffolds. In chapter 3, yttrium stabilized zirconia (YSZ) ceramic nanofibrous 
scaffolds were fabricated via electrospinning a mixture of its precursors and polyvinylpyrrolidone (PVP), followed by annealing of the obtained green fibers. These ceramic nanofirbous scaffolds behaved like a paper, having high flexibility and good fatigue behavior at macroscale, which is not common seen in traditional ceramic mats. Yet, the scaffolds retained their stiffness at the microscale. The surface roughness and grain size of YSZ nanofibers were dependent on the annealing processing parameter. Cell biology results demonstrated that the osteogenic differentiation and mineralization of seeded hMSCs was supported by the nanofibrous structure of YSZ scaffolds, in contrast to the known bioinert behavior of bulk YSZ. In addition, a better mineralization of hMSCs was observed on the scaffolds with a higher surface roughness. Altogether, these flexible ceramic scaffolds presented highly potential application for bone regeneration.

As a logical consequence of the findings of chapter 3 related to the effect of surface topography on mineralization, the influence of surface roughness on cell behaviors were further investigated. Whereas the influence of surface roughness and specific surface topographies on hMSCs activity is known in a number of two-dimensional substrates, limited knowledge is present when translating these studies to 3D scaffolds. In chapter 4, the surface nanoroughness of individual fibers in PEOT/PBT electrsopun scaffolds was varied via adjusting the relative humidity in the spinning chamber during electrospinning. The obtained scaffolds had the same polymer chemistry, similar porosity and pore size, and comparable fiber dimensions, yet a different surface roughness on individual fibers. The scaffolds having a rougher surface roughness of $71 \mathrm{~nm}$ had a higher protein adsorption than those having a surface roughness of $14 \mathrm{~nm}$. To investigate the influence of the surface roughness of individual fibers on cellular activities, hMSCs were seeded in scaffolds and cultured in osteogenic and chondrogenic differentiation media for 21 days. The higher surface roughness $(\mathrm{Ra}=71.0 \mathrm{~nm})$ promoted the expression of some osteogenic genes including osteopontin, bone morphogenetic protein 2 , and runtrelated transcription factor 2 , while the lower surface roughness ( $R a=14.3 \mathrm{~nm})$ showed to better up-regulate other osteogenic genes, such as bone sialoprotein, collagen type I alpha 1, and osteocalcin. The lower surface roughness $(\mathrm{Ra}=14.3 \mathrm{~nm})$ was more beneficial for chondrogenic genes expression at day 7. In summary, this research proposes a promising approach to fabricate electrospun scaffold with tailored topographical cues for skeletal differentiation of hMSCs.

The fibrous structure of native tissue has a complex and tissue dependent organization. For example, wavy patterns are observed in many connective tissues, while the collagen 
fiber orientation in cartilage, tendons, or ligaments is anisotropic. Thus, traditional electrospinning, which normally produce non-woven meshes, could not fairly meet all the biomimicry requirement for fabricating scaffolds in regenerative medicine. As such, new electrospinning techniques were described in chapter 5 and chapter 6 . Chapter 5 offers a simple and effective technique to fabricate wavy or crimped fibrous scaffolds. A wide range of crimped or wavy patterns could be achieved via tuning the processing conditions, including the orientation of the deposited fibers, additional sacrificial polymer layers, and the kind of thermally-shrinkable films. To the best of our knowledge, this research for the first time demonstrated the capability to generate multiscale crimped patterns spanning from fibers to fiber meshes through controlling the thickness of fiber layers. Cellular infiltration is one of the main concerns for electrospun scaffolds regarding their application in regenerative medicine. Here, hMSCs showed a more even cellular penetration in wavy electrospun fiber meshes compared to the non-wavy fiber meshes. Given the obtained wavy patterns mimicking the configuration observed in connective tissues, a mink lung epithelial cell line harboring a TGF- $\beta$ reporter construct was used to examine the effect of such topographical cues on TGF- $\beta$ signaling expression, which is a key signaling pathway involved in the development of several connective tissues. Compared to the non-wavy scaffolds, the wavy scaffolds promoted the expression of TGF- $\beta$ signaling.

Although solution electrospinning technique has been widely used for varied application, its inherent working principle of exploiting jet instability causes also the difficulty to deposit fibers in an accurate manner. This may hinder the further application of electrospinning where more hierarchical organization of nanofibers is needed. As such, chapter 6 shows a new technique for solution electrospinning with the capability to make electrospinning jet follow a stable path from the tip of the spinneret needle to the collector plate. Complex patterns and 3D multiscale structures with controllable porosity could be easily produced using this technique in contrast to traditional electrospinning. Using this new technique, a scaffold that mimics articular cartilage with its distinct multi-zone morphology was fabricated. To estimate their potential application in cartilage tissue engineering, hMSCs was seeded in scaffolds in chondrogenic differentiation medium for 21 days. SEM analysis, histological and immunostaining results demonstrated that the scaffold directed tissue organization and fibril matrix orientation in a zone-dependent way. Moreover, gene expression results revealed that chondrogenic markers including Sox9 and aggrecan were significant upregulated in the scaffolds compared to traditional 
electropsun mesh. Overall, the research here provided a simple way to fabricate customized fibrous scaffolds for regenerative medicine, especially those for which anisotropy is of importance.

In chapters 7, themes are summarized and discussed in relation to the results presented in this thesis and a few possible ideas for future work. Finally, chapter 8 describe the valorization potential of the research presented in this thesis. 


\section{Acknowledgements}

First of all, I want to thank Clemens van Bliterswijk for providing me with the opportunity to work in your group. I enjoyed the freely academic atmosphere in your department. Although we do not have much talk, I have heard the wonderful stories of your career. Your passion and the spirit of challenging yourself will inspire my future career.

Lorenzo, congratulations! You realized your dream to be a full professor before your 40 age. You are always patient with me, encourage me, and stand by my side even though sometimes I made you feel awkward, such as missing the deadline, making little progress on projects, and receiving complaints about my unsuitable behavior in labs. Your door is always open, which is a PhD student could hope for. I have learned a lot from you. It is not only lay on your knowledge in tissue engineering but also your personality such as enthusiasm, hard-working, and social ability. I am confident all these qualities will benefit a lot my future career. I hope we will have occasions in future to collaborate and to maintain contact.

Roman, my second daily supervisor, thanks a lot for many inspiriting discussions we had in the past few years. You are a fountain of technical idea. Pamela, Yan, Aart, Stefan, Vanessa, Aurélie, Sabine, and Matt thank you for your helpful discussion during the TR group meetings or Merln colloquiums.

To Ivan, thank you for the joy, yoga and laugher you bring to us. We often make fun at each other which make me ignore the trouble from work. Thank you for being my paranymphs.

Bach, you are another guy fully equipped with knowledge. And always be kind to give me a hand.

Gerard, it is my pleasure to collaborate with you, from which we came out a high potential paper. I remembered the funny moment when we pretend to enjoy smoking while leaning on each other during the party night after the TERMIS Conference.

Feb and Niloofar, both of you are always generous to help me with the solution to the problems that I had from work. 
Erik, Wim, and Ziryan, thank you for helping me with understanding annoying letters which were often in Dutch. You have saved me from a hefty fine so that I can stand before you now without being naked(:).

Pinak, I like the milk tea you made. It is pleasure to chat with you on our way home. I wish we will keep our friendship in future.

Afonso and Danielle, it is a good experience to supervise you, which helped me to better understand what will expect me in my future career. Both of you are PhD students in Merln institute as well. I wish you both enjoy every moment of your life in Merln.

Jeroen Leijten, thank you for your helpful discussions on chondrogenisis of hMSCs and your experience in applying for postdoc fellowship.

I could not have finished my thesis without the input and help of all my other colleagues and collaborators. To just name a few: Carlos, Paul, David Barata, Ravi, Angad, Huey, Abhishek, Andrea Calore, Andrea Diluca, David Gomes, Jip, Khadija, María, Rabeil, Vahid, Aliaksei, Nick, Steven, Daniel, Víctor, Anne, Giuseppe, Nicky, Denis, Christine, Marloes, Romina, Anouk, Audrey, Tom, , Carola, Angad, Chiaria, Parthiban, Elahe, JOÃO, Corina, Tom, and many, many more who have ever worked in department of tissue regeneration and Merln institute since I arrived at Netherlands.

I also would like to acknowledge my chinese colleagues and friends. 靖微, 感谢你在我 第一天来荷兰时, 去火车站接我。在接下来日子里, 你就像一个姐姐一样热情帮助我, 带 领我去参加活动和聚会, 帮我尽快适应这边的生活。每当我在工作上遇到困难时, 或与老 板沟通不顺畅里, 你会 “恨铁不成钢” 地谆谆规劝我多加努力。你会经常邀请我去你家品 尝你做的可口饭菜。所有的这些, 不管多少年以后想起来, 内心总会倍感温暖, 心存感激。 忠华, 跟你一起打球一起聊天, 让我受益非浅。你那特有的东北腔总是能把大伙逗得开怀 大笑。与你一起搭挡打扑克很爽, 特别是让小斌带上 “千年老二” 的感觉更棒, 哈 哈 。小奎, 我的小伙伴, 我们一起健身, 一起打各种球, 一起打牌, 一起逛”OMA”, 一起聚餐, 基本上有我的出没的地方, 都有你强壮的身影, 总之各种的在一起, 能认识一 个像你这样聪明的小伙伴我感到很荣幸。祝你跟熊总早生贵子, 加油哦! 小斌和蕾蕾, 我 们经常在办公室二楼那银灰色的桌子上一起吃午饭, 聊八卦, 聊课题, 聊生活。工作之余 还经常一起聚会, 一起打球。每当这个时候, 我总会感到很放松。恭喜你们生了个黄美兰 沈, 又生了个黄美承康, 祝他们健康快乐地成长。小斌, 跟你一起打牌很爽前提是不要跟 你对家。王荣, 首先感谢你作为我的 paranymphs。其他朋友像割非菜一样走了一茬又一 茬, 各奔东西, 你是我目前在荷兰认识的比较熟悉的 “长老级人物” 之一, 从在屯特时共享 
实验室, 到现在共享导师。祝你未来路越走越顺。曾亦健老乡和大荣姐, 怀念一起聚会的 日子, 祝你们家曾高兴茁壮成长。

路路、王否、杜䒯、海荣、宋卫余、小华、我依旧清晰记得, 刚来荷兰不久, 大伙一起 结伴旅行过的日子, 那时候大家意气风发, 有种恨不得踏遍整个欧洲旮昌的样子, 那段青 葱岁月也是我最难忘的时光。卓拉，感谢你去美国开会时帮我买了个土豪金 iphone5S,让 我从此变成 “土豪”, 还有你像个女汉子一样帮我抬冰箱。

赵永, 我们一起聊天谈心、一起旅行、一起聚餐, 希望你在未来的日子里一切顺利, 尽快找到工作和心仪的另一半。

蓝天, 我们经常端着咖啡, 做在沙发上从天上星星谈到各自的项目, 每次聊天都很享受。 王毅啊, 我们在一个屋檐下生活了很长一段时间, 那时跟老杨经常一起请客, 那个场面 其乐融融！感谢你和爱洁在我快要离开恩斯赫德时, 给我提供住宿。

家平大哥和天宇, 我们经常在办空室里一起吃饭, 一起聊天, 感谢遇到你们。祝天宇你 实验顺利, 天天开心。

林逸聪, 很高兴来马城后能认识你这个邻居。有一段时间我们常常一起跑步, 一起自习 到深夜, 一起顶着风冒着雨回家。在那段岁月里, 我感到很快乐也很充实。

张明和辛颖, 祝你们在荷兰的生活一切顺利。

我还要感谢以下这些人, 因为他们的出现让我的生活更加丰富多彩。他们是：老杨、孙 醒吾、陈文龙、郭正潮、刘善秋、马敬红、徐红、黄颖、张利杰、冯雪凌、隋晓锋和高玉 英夫妇、黎瑶、付姚、张凯欢和程思佳、王梦姣、李果、孙兰英、牛柯南、赵俊锋夫妇、 乔奇、李斌龙、侯东爽、林怡远、吴凌、马斌、刘俊。一路走来, 要感恩的人和事太多, 在此不能一一列举, 衷心希望大家都能幸福美满。

In the end, I would like to thank my family for making all of this possible. 爸爸妈妈哥哥 妹妹, 你们无尽的爱给了我勇往直前的信心; 不管任何时候, 高潮或低谷, 你们总是在背 后默默无闻地支持我, 给了我无限的宽慰。大爱无声！！还要感谢我的女朋友敏敏, 感谢 有你陪伴的日子。

In case I have missed anyone, sorry and thank you!

Honglin Chen

March $8^{\text {th }}, 2017$ 


\section{Biography}

Honglin Chen was born and raised in a beautiful village of Putian, China. In the fall of 2004, he began his studies at Minjiang University where he received training in in the principle of Chemical and Chemistry Engineering. In 2008, he continued his studies in Department of Chemistry at East China Normal University, Shanghai. After three years, he graduated with a Masters of Polymer Chemistry and Physics. His dissertation for Master degree is entitled "electrospun chitosan-polycaprolactone materials and their application for tissue engineering". In the fall of 2011, he started his doctoral studies in the Tissue Regeneration group of Clemens van Blitterswijk, University of Twente, Netherlands, under the daily supervision of Prof. Lorenzo Moroni and Dr. Roman Truckenmüller. He gained his knowledge in designing scaffolds for guidance skeletal differentiation of human mesenchymal stromal cells, as well as his age. In the December of 2014, the Tissue Regeneration Group found its bright future in Maastricht University. He decided to move from Enschede to Maastricht following his supervisors and continued to pursue his $\mathrm{PhD}$ degree in the Complex Tissue Regeneration. 


\section{List of Publications}

This thesis is based on the following publications:

\section{Peer Reviewed Papers:}

Honglin Chen, Matteo Gigli, Chiara Gualandi, Roman Truckenmüller, Clemens van Blitterswijk,Nadia Lotti, Andrea Munari, Maria Letizia Focarete, Lorenzo Moroni. Tailoring chemical and physical properties of fibrous scaffolds from block copolyesters containing ether and thio-ether linkages for skeletal differentiation of human mesenchymal stromal cells. Biomaterials. 2016; 76: 261-272.

Gerard Cadafalch Gazquez ${ }^{[+]}$, Honglin Chen ${ }^{[+]}\left({ }^{[+]}\right.$co-first authorship), Sjoerd A. Veldhuis, Alim Solmaz, Carlos Mota, Bernard A. Boukamp, Clemens A. van Blitterswijk, Johan E. ten Elshof, and Lorenzo Moroni. Flexible Yttrium-Stabilized Zirconia Nanofibers Offer Bioactive Cues for Osteogenic Differentiation of Human Mesenchymal Stromal Cells. ACS Nano. 2016, 10 (6), 5789-5799.

Honglin Chen, Xiaobin Huang, Minmin Zhang, Febriyani Damanik, Mattew B. Baker, Anne Leferink, Huipin Yuan, Roman Truckenmuller, Clemens van Blitterswijk, Lorenzo Moroni. Tailoring surface nanoroughness of electrospun scaffolds for skeletal tissue engineering.(In submission).

Honglin Chen, Danielle Ferreira Batista, Roman Truckenmuller, Clemens van Blitterswijk, Lorenzo Moroni. Tailoring Crimp Pattern of Electrospun Fibers by using thermal shrinkage film. (manuscript in preparation).

Honglin Chen, Afonso Malheiro, Paul Wieringa, Carlos Mota, Roman Truckenmuller, Clemens van Blitterswijk, Lorenzo Moroni. Fabrication of scaffold mimicking the architecture of articular cartilage using direct-write electrospinning. (In submission).

G. Cadafalch Gazquez, Honglin Chen, Lorenzo. Moroni, Bernard A. Boukamp, Johan E. ten Elshof. $\beta$-TCP Nanofiber scaffolds for bone regeneration with fine unidirectional grains. (manuscript in preparation). 


\section{Book Chapter:}

Honglin Chen, Roman Truckenmüller, Clemens van Blitterswijk, Lorenzo Moroni. (2013). Fabrication of nanofibrous scaffolds for tissue engineering applications. In: A.K. Gaharwar \& S. Sant \& M.J. Hancock \& A.A. Hacking (Eds.), Nanomaterials in tissue engineering: Fabrication and applications. Series in Biomaterials (56). Woodhead Publishing, 158 182. ISBN 9780857095961

\section{Other Publications:}

Honglin Chen, Jin Huang, Jiahui Yu, Shiyuan Liu, Ping Gu. Electrospun chitosan-graftpoly ( $\varepsilon$-caprolactone)/poly ( $\varepsilon$-caprolactone) cationic nanofibrous mats as potential scaffolds for skin tissue engineering. Int J Biol Macromol. 2011; 48:13-19.

Honglin Chen, Jin Huang, Jiahui Yu, Shiyuan Liu, Ping Gu. Electrospun chitosan-graftpoly ( $\varepsilon$-caprolactone)/poly ( $\varepsilon$-caprolactone) nanofibrous scaffolds for retinal tissue engineering. Int $\boldsymbol{J}$ Nanomedicine. 2011; 6: 453-461.

Kongrong Duan, Honglin Chen, Jin Huang, Jiahui Yu, Shiyuan Liu, Daxin Wang, Yaping Li. One-step synthesis of amino-reserved chitosan-graft-polycaprolactone as a promising substance of biomaterial. Carbohydr Polym.2010 80(2):498-503

\section{Conference Abstracts:}

Honglin Chen, Afonso Malheiro, Paul Wieringa, Carlos Mota, Roman Truckenmuller, Clemens van Blitterswijk, Lorenzo Moroni. Fabrication of scaffold mimicking the architecture of articular cartilage using direct-write electrospinning. International Conference on Biofabrication (Oral, Utrecht, The Netherlands, 2015)

Honglin Chen, Xiaobin Huang, Minmin Zhang, Febriyani Damanik, Roman Truckenmuller, Clemens van Blitterswijk, Lorenzo Moroni, Tailoring surface nanoroughness of electrospun scaffold for skeletal tissue engineering. Netherlands society for biomaterials and tissue engeneering (NBTE) conference(Oral, Lunteren, Netherlands, 2015)

Honglin Chen, Danielle Ferreira Batista, Roman Truckenmuller, Clemens van Blitterswijk, Lorenzo Moroni. Tailoring Crimp Pattern of Electrospun Fibers by using thermal shrinkage film. European Society for Biomaterials (ESB) Conference(Oral, Liverpool, UK 2014)

Honglin Chen, Chiara Gualandi, Matteo Gigli, Marialetizia L. Focarete, Nadia Lotti, Roman Truckenmuller, Clemens V. Blitterswijk, Lorenzo Moroni. Bioactive Elastomers for 
Osteogenic Differentiation of Human Mesenchymal Stem Cells. Termis-EU Conference (Poster, Genova, Italy 2014).

Honglin Chen. Gerard Cadafalch Gazquez, Sjoerd A. Veldhuis, Alim Solmaz, Carlos Mota, Bernard A. Boukamp, Clemens A. van Blitterswijk, Johan E. ten Elshof, and Lorenzo Moroni. Flexible ceramic nanofiber for osteogenic differentiation of human mesenchymal stem cells. Netherlands society for biomaterials and tissue engeneering (NBTE) conference (Poster, Lunteren, Netherlands, 2012). 